\title{
Advanced NMR Techniques for the Investigation of Small Molecules in Solution
}

\author{
Dissertation \\ zur Erlangung des mathematisch-naturwissenschaftlichen Doktorgrades \\ „Doctor rerum naturalium“ \\ der Georg-August-Universität Göttingen
}

im Promotionsprogramm Chemie

der Georg-August University School of Science (GAUSS)

vorgelegt von

Thomas Niklas

aus Olpe

Göttingen, 2017 


\title{
Betreuungsausschuss
}

Prof. Dr. Dietmar Stalke, Institut für Anorganische Chemie

Prof. Dr. Franc Meyer, Institut für Anorganische Chemie

\section{Mitglieder der Prüfungskommission}

\author{
Referent: $\quad$ Prof. Dr. Dietmar Stalke \\ Korreferent: Prof. Dr. Franc Meyer
}

weitere Mitglieder der Prüfungskommission

Prof. Dr. Ricardo A. Mata, Institut für Physikalische Chemie

Prof. Dr. Manuel Alcarazo, Institut für Organische Chemie

Prof. Dr. Konrad Koszinowski, Institut für Organische Chemie

Dr. Michael John, Institut für Organische Chemie

Tag der mündlichen Prüfung: 20.06.2017 
für meine Familie 
I like the cold weather. It means you get work done.

Noam Chomsky 
Parts of Sections 2.2 and 2.3 of this thesis have been published as:

1. T. Niklas, D. Stalke, M. John, "Single-shot titrations and reaction monitoring by slice-selective NMR spectroscopy" Chem. Commun., 2015. 51, 1275-1277.

Parts of Section 4.2 of this thesis have been published as:

2. T. Niklas, C. Steinmetzger, W. Liu, D. Zell, D. Stalke, L. Ackermann, M. John, "Determination of the Relative Configuration of $\beta$-Amino Acid Esters Based on Residual Dipolar Coupling." Eur. J. Org. Chem. 2015, 2015, 6801-6805. 


\section{Table of Contents}

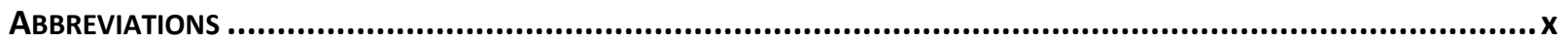

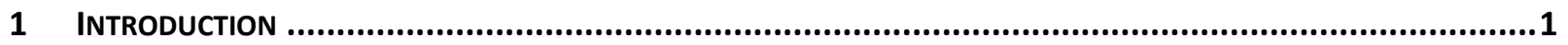

$1.1 \quad$ Lithium Organic Compounds …………………………………………………………………….... 1

1.1.1 Applications ............................................................................................................................ 1

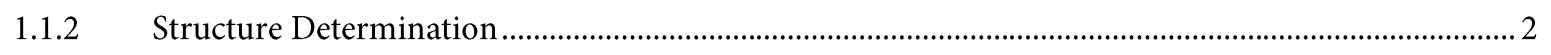

1.2 Slice-Selective Excitation...............................................................................................................

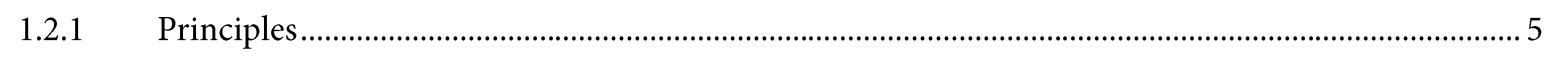

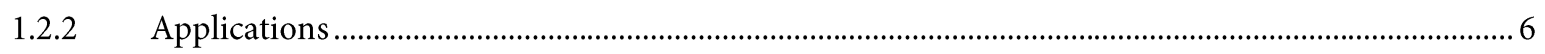

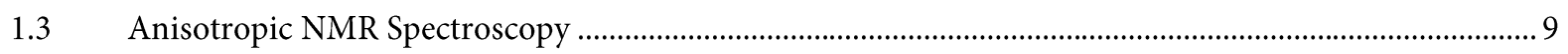

1.3.1 Residual Dipolar Couplings ...............................................................................................................

1.3.2 Residual Quadrupolar Couplings ..................................................................................................... 15

1.3.3 Alignment Techniques ................................................................................................................... 19

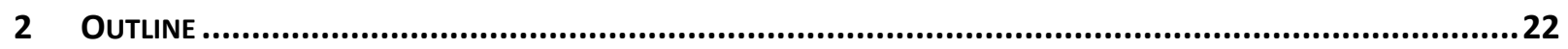

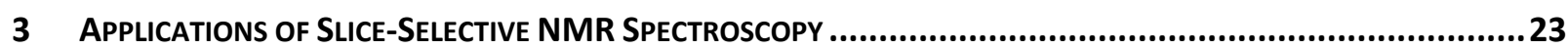

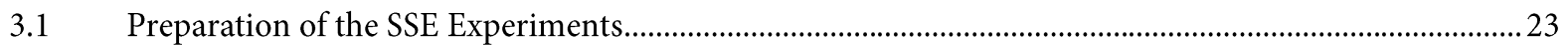

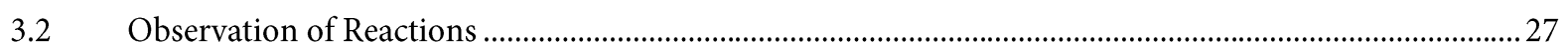

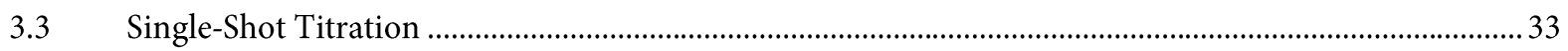

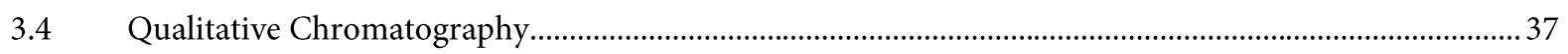

3.5 Summary and Outlook Slice-Selective Excitation .......................................................................................... 43

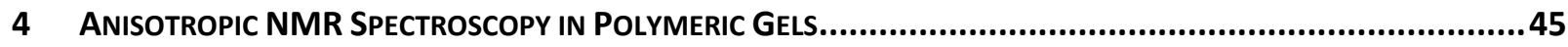

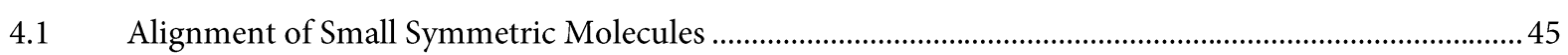

4.1.1 Molecular Dynamics Simulations .................................................................................................. 51

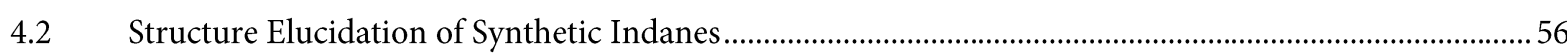

4.2.1 Structural Error Analysis.................................................................................................................65

4.3 Quantitative Analysis of Residual Quadrupolar Couplings ..................................................................69

4.4 Summary and Outlook Anisotropic NMR Spectroscopy ……………………………………………….. 78

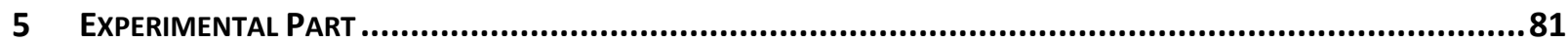

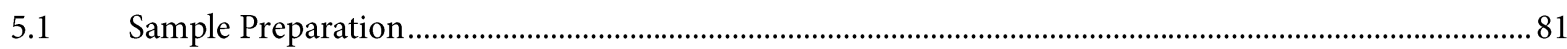

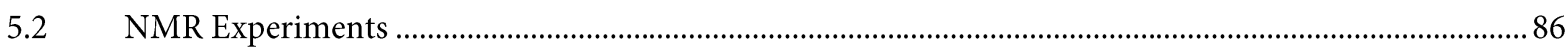

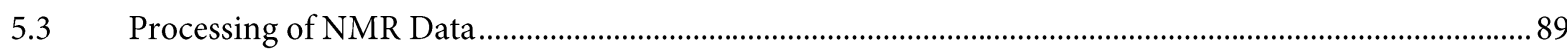

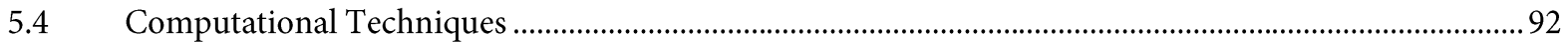

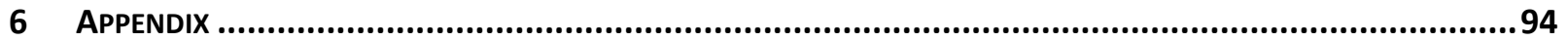

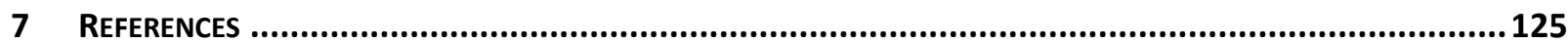

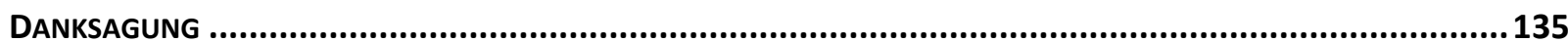

CURRICULUM VITAE 


\section{AbBREVIations}

\begin{tabular}{|c|c|}
\hline Asp & aspartic acid \\
\hline$a x$ & axial \\
\hline $\mathrm{BuLi}$ & butyllithium \\
\hline $\mathrm{CDF}$ & combined distribution function \\
\hline CIP & contact ion pair \\
\hline CLIP-HSQC & clean-inphase HSQC \\
\hline COSY & correlation spectroscopy \\
\hline CPMG & Carr-Purcell-Meiboom-Gill \\
\hline CpLi & cyclopentadienyllithium \\
\hline $\mathrm{DCM}$ & dichloromethane \\
\hline DFT & density functional theory \\
\hline DHPC & dihexanoyl phospatidylcholine \\
\hline DMPC & dimyristoyl phosphatidylcholine \\
\hline DOSY & diffusion ordered spectroscopy \\
\hline DMG & directing metallation group \\
\hline DVB & divinylbenzene \\
\hline EFG & electric field gradient \\
\hline ESP & electrostatic potential \\
\hline$e q$ & equatorial \\
\hline FID & free induction decay \\
\hline His & histidine \\
\hline HMQC & heteronuclear multi quantum coherence \\
\hline HSQC & heteronuclear single quantum coherence \\
\hline MD & molecular dynamics \\
\hline MeLi & methyllithium \\
\hline $\mathrm{Me}_{2} \mathrm{O}$ & dimethylether \\
\hline MRI & magnetic resonance imaging \\
\hline MW & molecular weight \\
\hline NMR & nuclear magnetic resonance \\
\hline NOESY & nuclear Overhauser enhanced spectroscopy \\
\hline OPLS & optimized potential for liquid simulations \\
\hline PAA & p-azoxyansiol \\
\hline PAS & principal axis system \\
\hline PBA & polybutylacrylate \\
\hline PBBA & $p$-butoxy-benzoic acid \\
\hline
\end{tabular}




\section{Abbreviations}

PMDTA

PiLi

PS

Q.E.COSY

QSC

RCSA

RDC

RI

RQC

SAG

SDF

SSE

SVD

$T_{1} \mathrm{IR}$

THF

TMEDA

Tyr

Val

XRD
$N, N, N$ ', $N^{\prime \prime}, N^{\prime \prime}$-pentamethyldiethylenetriamine

piperidinyllithium

polystyrene

quadrupolar exclusive COSY

quadrupolar splitting constant

residual chemical shift anisotropy

residual dipolar coupling

rapid injection

residual quadrupolar coupling

strain induced alignment in a gel

spatial distribution function

slice-selective excitation

singular value decomposition

$T_{1}$ inversion recovery

tetrahydrofurane

$N, N, N$ ', $N$ '-tetramethylethylenediamine

tyrosine

valine

X-ray diffraction 



\section{INTRODUCTION}

High resolution nuclear magnetic resonance (NMR) spectroscopy is arguably the most powerful and versatile method for the observation of molecules in solution. Applications range from detailed structure elucidation to the characterization of dynamic reaction processes with seemingly infinite possibilities in between. This thesis focuses on the development of new methods in NMR spectroscopy of small molecules with an emphasis on lithium organic compounds.

\subsection{Lithium Organic Compounds}

\subsubsection{Applications}

The outstanding reactivity of lithium organic compounds is frequently highlighted and requires specific working procedures under inert conditions as introduced by Schlenk and Holtz in 1917. ${ }^{[3]}$ This reactivity reflects in the ability to form (thermodynamically challenging) $\mathrm{C}-\mathrm{C}$ bonds which in turn has expedited research ever since. Nowadays, lithium organics resemble a versatile class of chemical reagents, and applications range from the industrial production of rubber ${ }^{[4]}$ to modern regio- and stereoselective synthesis of pharmaceuticals. ${ }^{[5]}$ For instance, the directed ortho-lithiation is an omnipresent procedure in synthesis that combines selectivity and reactivity towards a broad field of substrates (Scheme 1-1).

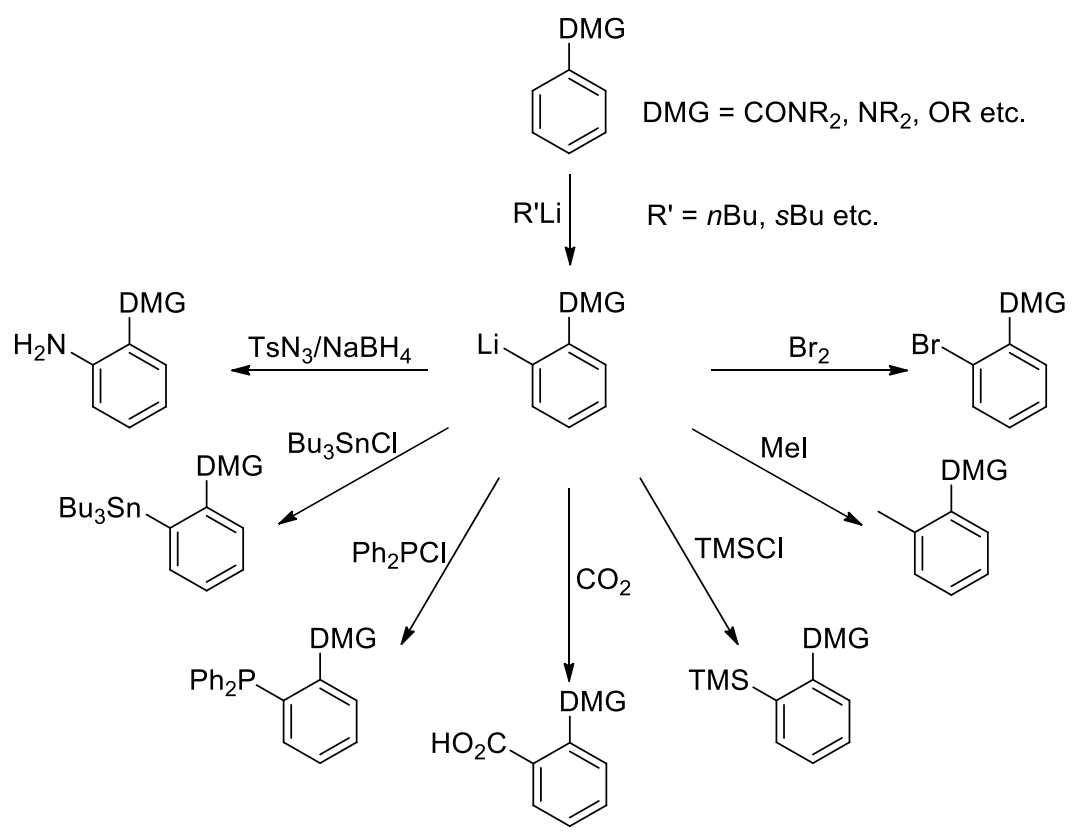

Scheme 1-1: Directed ortho-lithiation of an aromatic compound containing a directing metalation group (DMG) and subsequent conversions with $\mathrm{TsN}_{3} / \mathrm{NaBH}_{4}{ }^{[6]}, \mathrm{Bu}_{3} \mathrm{SnCl}^{[7]}, \mathrm{Ph}_{2} \mathrm{PCl}^{[7]}, \mathrm{CO}_{2}{ }^{[8]}, \mathrm{TMSCl}^{[0]}, \mathrm{MeI}^{[10]}$ and $\mathrm{Br}_{2}{ }^{[11]}$.

Gilman $^{[12]}$ and Wittig ${ }^{[13]}$ independently discovered that aromatic compounds bearing directing metalation groups (DMGs) such as amides, tertiary amines or ethers can be selectively lithiated in ortho 
position. These lithiated compounds can be converted in subsequent electrophilic substitution reactions.

Despite their routine usage, lithium organic synthesis protocols are largely based on the empirical determination of reaction behaviour, rather than the derivation of mechanisms from first principles. Yet, rational design of synthetic strategies must be an objective of modern chemical research and can only be achieved by correlating structure and reactivity.

\subsubsection{Structure Determination}

\section{Structure Determination in Solid State}

Comprehensive structure determination in the solid state has long been aggravated by the sensitive, pyrophoric nature of lithium organic compounds. This changed especially with the development of cryo-techniques for single crystal X-ray diffraction (XRD) in the early $90 \mathrm{~s}^{[14]}$ Since then, a plethora of $3 \mathrm{D}$ structures with variations in coordination number, ligand identity and molecular symmetry has been obtained which are reviewed in detail elsewhere. ${ }^{[15]}$ Seminal studies from Stalke et al. revealed basic reoccurring motifs that are formed by the lithium atoms. ${ }^{[16]}$ Most prominent examples are the octahedral $n$-butyllithium $(n \mathrm{BuLi})_{6}$ or tetrahedral tert-butyllithium $(t \mathrm{BuLi})_{4}$ structures (Figure $\left.1-1\right)$.
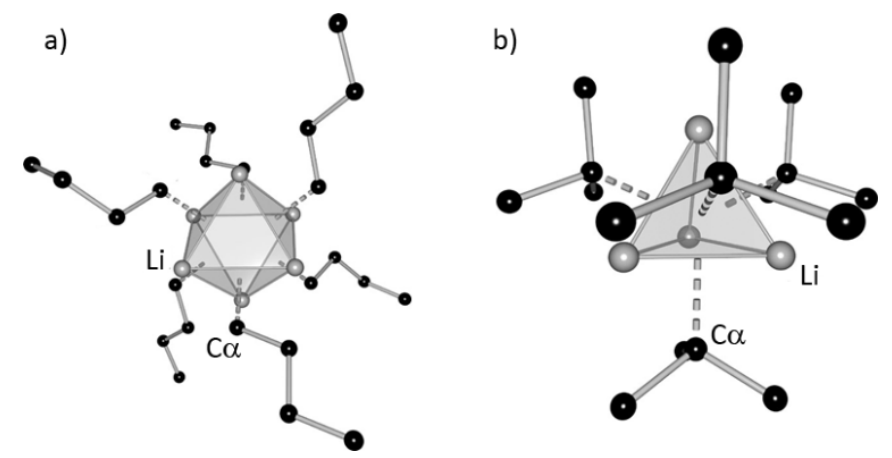

Figure 1-1: Hexameric $n$-butyllithium $(n \mathrm{BuLi})_{6}(\mathrm{a})$ and tetrameric tert-butyllithium $(t \mathrm{BuLi})_{4}$ (b) as basic reoccurring structural motif of lithium organics in the solid state. Hydrogen atoms are omitted for clarity. Reprinted with permission from reference [15c]. Copyright 2014 Wiley VCH.

It is a common perception that the formation of these polyhedral aggregates is largely owed to the ionic character of the $\mathrm{Li}-\mathrm{C}$ bond. ${ }^{\mathrm{a}}$ Hence, the polarity of solvents exerts a huge influence on the aggregation: it has frequently been shown that charge-stabilizing, polar solvents or specific addition of polydentate, electron-donating Lewis bases enforces the disaggregation of polyhedral aggregates into smaller subunits (Figure 1-2).

\footnotetext{
${ }^{a}$ These structural motifs are usually described as contact ion pairs. A further, highly interesting, class of organometallic compounds is classified as solvent separated ion pairs. These compounds are characterized through distinct, solvated cation and anion subunits. Although, these molecules feature a range of interesting properties, this work will not further elaborate on their characteristics and rely on studies given elsewhere. ${ }^{[1]]}$
} 

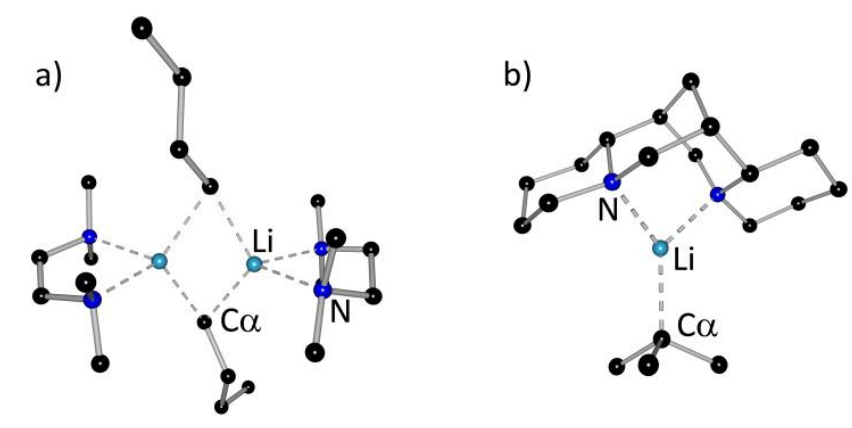

Figure 1-2: Dimeric $n \mathrm{BuLi}$ TMEDA aggregate (TMEDA.nBuLi) $)_{2}$ (a) and monomeric $t \mathrm{BuLi}(-)$-sparteine aggregate [(-)-sparteine.tBuLi] (b). Hydrogen atoms are omitted for clarity. Reprinted with permission from reference [15c]. Copyright 2014 Wiley VCH.

For example, $N, N, N^{\prime}, N^{\prime}$-tetramethylethylenediamine (TMEDA) allows the preparation of dimeric $\left(\right.$ TMEDA.nBuLi) ${ }_{2}{ }^{[18]}$ (Figure 1-2 a), and chiral (-)-sparteine even yields a monomeric complex with $t \mathrm{BuLi}^{[19]}$ (Figure 1-2 b). The latter one enables asymmetric reaction steps as required in the synthesis of pharmaceuticals. ${ }^{[5,20]}$ Accordingly, it has been well documented that the reactivity of lithium organics increases by switching from apolar solvents (e.g. toluene, $n$-hexane) to polar solvents (e.g. diethylether, tetrahydrofurane) to solvents with additional donor bases (e.g. TMEDA, (-)-sparteine). ${ }^{[21]}$ From this behaviour a structure-reactivity relationship of lithium organics has been deduced, which states that the increase in reactivity is achieved through disaggregation. ${ }^{\mathrm{a}}$

Due to adverse molecular properties, single crystals may not always be available. In these cases contributions have perpetually been made by powder XRD; e.g. the tetrameric methyllithium structure $(\mathrm{MeLi})_{4}$ was determined by Weiss et al. from powder data in $1964 .{ }^{[23]}$ Similarly, solid state NMR spectroscopy has regularly complemented XRD with a range of valuable structural information (vide infra). ${ }^{[24]}$ Yet, it must be emphasized that chemical syntheses are primarily performed in solution. Since it cannot be assured that structural motifs in solution and solid state coincide, liquid state techniques must support or ensure results from XRD, solid state NMR and likewise. ${ }^{b}$

\section{Structure Determination in Solution}

It is obvious that the crucial role of solution structure determination for reaction design is widely recognized and has boosted methods development especially in the field of NMR techniques. Among these methods is heteronuclear Overhauser spectroscopy which has been employed for the determination of distances between $\mathrm{H}$ and Li e.g. by Bauer ${ }^{[26]}$ or Gschwind ${ }^{[27]}$. Very recently, Oulyadi and co-workers showed how scalar Li-Li coupling constants can be extracted and utilized to obtain structural information about mixed lithium structures. ${ }^{[28]}$ Yet, the most widely used NMR techniques are rapid-injection $^{[29]}$ (RI) and diffusion-ordered spectroscopy ${ }^{[30]}$ (DOSY).

\footnotetext{
${ }^{a}$ This concept is challenged with several exceptions. ${ }^{[22]}$ Usually, these divergent cases can be explained through additional counteracting effects, such as disaggregation and extensive shielding of the polarized carbon lithium bond. Hence, the structure-reactivity relationship of lithium organics still remains a valuable and successfully applied hypothesis.

${ }^{b}$ Additionally, state-of-the-art structure elucidation and reactivity studies of lithium organics heavily depend on quantum mechanical calculations. Several excellent reviews focus on this field of research. ${ }^{[25]}$
} 
RI NMR is usually performed to deduce the kinetic behaviour of analytes. This technique is based on an apparatus that usually injects the substrate into a solution containing the reagent and achieves a full and homogeneous sample mixing in the timescale of milliseconds. ${ }^{[29]}$ These rapid injections are instantaneously followed by the acquisition of spectra for a detailed monitoring of the reaction progress between both substrates. Variations in concentration and temperature then allow an ample deduction of the kinetic behaviour of the analytes as detailed in several excellent reviews. ${ }^{[31]}$

DOSY enables the highly accurate determination of self-diffusion coefficients $D$ through NMR techniques. ${ }^{[30]}$ The dependency of diffusion coefficients on molecular hydrodynamic radii is given through the Stokes-Einstein equation. ${ }^{[32]}$ By furthermore correlating the hydrodynamic radii with molecular weights $(M W)$ it is possible to achieve a linear connection between the logarithmic diffusion coefficient $\log (D)$ and the logarithmic molecular weight $\log (M W) \cdot{ }^{[33]}$ Therefore, preparation of calibration curves from model compounds enables DOSY MW estimation of unknown analytes from their diffusion coefficients (Figure 1-3).

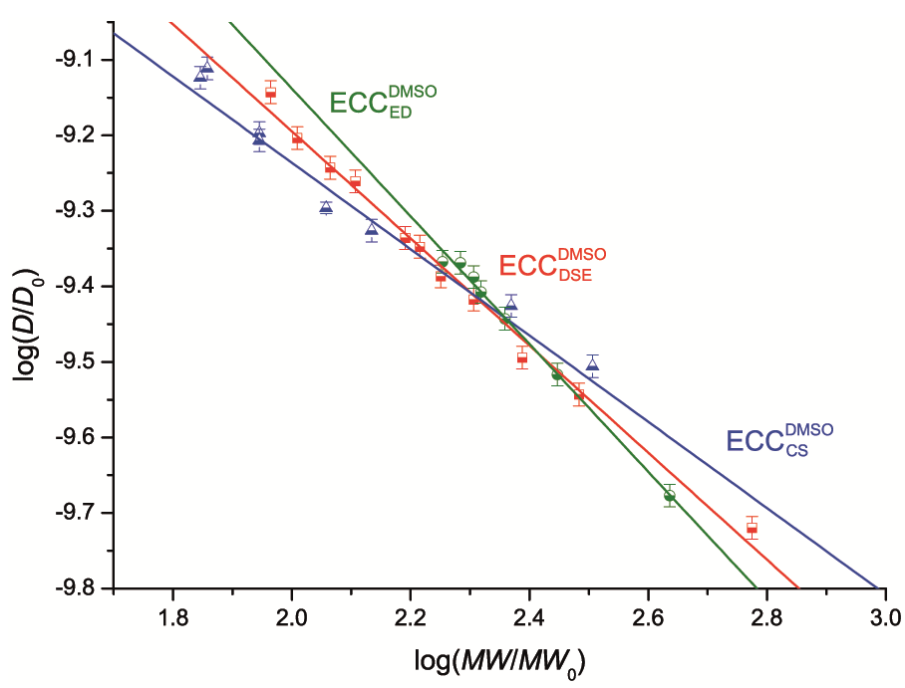

Figure 1-3: Straight lines represent the linear correlation between logarithmic diffusion coefficient $\log \left(D / D_{0}\right)$ as determined from DOSY experiments and logarithmic molecular weight $\log \left(M W / M W_{0}\right)$ of a range of simple organic molecules in dimethylsulfoxide (DMSO). These external calibration curves (ECCs) were additionally categorized according to the shape of the molecules that are employed: Expanded discs (ED, green), dissipated spheres and ellipsoids (DSE, red) and compact spheres (CS, blue). Reprint with permission from reference [34]. Copyright 2016 Wiley VCH.

These estimated weights can then be compared with hypothetical weights of a range of possible aggregates. Hence, differentiation e.g. between monomeric and dimeric species can be made with ease. ${ }^{[35]}$ Recently, Neufeld et al. and Bachmann et al. ${ }^{[34]}$ have made significant advancements through adapted, universally applicable calibration curves (Figure 1-3). ${ }^{[36]}$

Although these techniques have proven to be highly successful, there is a constant challenge to develop further elaborate NMR techniques and overcome remaining limitations. For instance, various possible aggregates (structural proposals generated from combinations of lithium organic compounds and coordinating donor bases) may have very similar $M W$ s and are (unlike monomeric and dimeric species) hardly distinguishable via DOSY MW estimation. In these cases complementary (NMR) techniques 
have to provide further evidence on structural features to verify/falsify the proposals. Among these methods are slice-selective excitation ${ }^{[37]}$ (SSE) and anisotropic NMR spectroscopy ${ }^{[38]}$, which are detailed in the following section.

\subsection{Slice-Selective Excitation}

\subsubsection{Principles}

SSE is an NMR technique to obtain spatial resolution while acquiring high-resolution spectra. It can be readily illustrated through the Zeeman splitting of nuclei with spin $I=1 / 2$ in the presence of a locally dependent magnetic field $\vec{B}_{z}$ (Figure 1-4).

a)

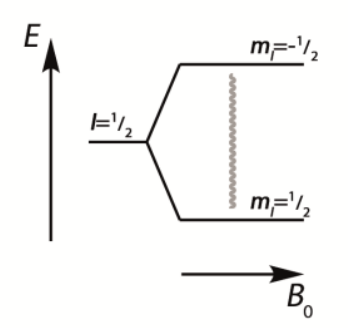

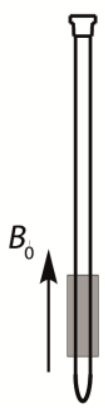

b)

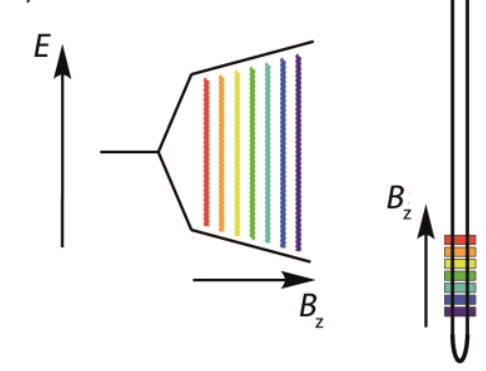

Figure 1-4: a) Zeeman splitting of a spin 1/2 nucleus in a constant magnetic field $B_{0}$ leads to the excitation of the whole active sample volume (grey) via a hard pulse. b) A spatially dependent magnetic field $B_{z}$ and frequencyshifted soft pulses allow the excitation of discrete slices (colored).

Figure 1-4 a) shows that in a static magnetic field $\vec{B}_{0}$ a single hard pulse with matching frequency excites the full active volume of the sample. The application of a field gradient along $z$ (Figure 1-4 b) leads to a locally dependent magnetic field $\vec{B}_{z}$ which in turn causes a locally dependent Zeeman splitting. This enables excitation of sample slices through shaped soft pulses with varying frequencies that match the energy splitting of the desired slice.

The basic SSE pulse sequence is simple and robust and therefore easily implemented for standard liquid state NMR instruments (Figure 1-5).

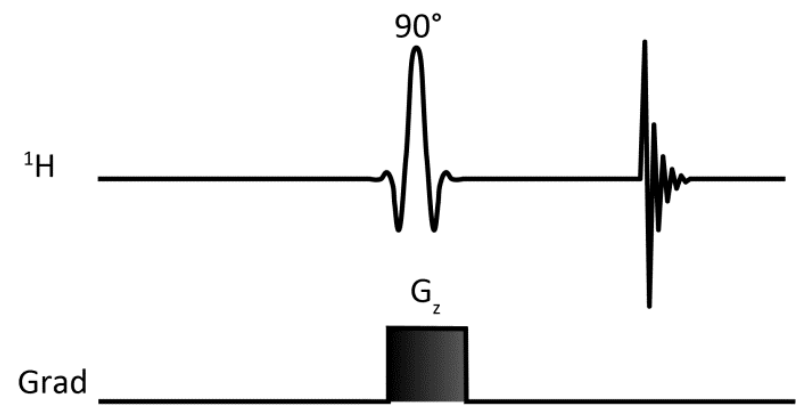

Figure 1-5: Basic SSE pulse sequence consisting of a soft $90^{\circ}$ shaped pulse that is delivered simultaneously with a pulsed gradient $G_{z}$. 
The pulse program is composed of a frequency-encoding pulsed magnetic field gradient $G_{z}$ and a simultaneous soft $90^{\circ}$ shaped pulse followed by the acquisition of the signal. The strength of the gradient $G_{z}$ effects the spatially dependent magnetic field $B_{z}$. Thus, pulses with frequency offsets $v_{z}$ excite nuclei with gyromagnetic ratio $\gamma$ of slices $z$ where $z^{\prime}=0 \mathrm{~mm}$ is at the centre of the gradient coil (1-1).

$$
v_{\mathrm{z}}=\frac{\gamma \cdot G_{\mathrm{z}} \cdot z^{\prime}}{2 \pi}
$$

Negative $z^{\prime}$ refer to the slices below and positive $z^{\prime}$ to the slice above. Note that in the following chapters $z$ substitutes $z$ ' where $z=0 \mathrm{~mm}$ refers to the topmost slice and slices below are numbered consecutively. Additionally, $v_{z}$ was incremented in steps to excite slices at $0 \mathrm{~mm}, 1 \mathrm{~mm}, 2 \mathrm{~mm}$, etc. for convenience, these slices are referred to as slice $0,1,2$, etc. i.e. metric unit can be dropped. Besides frequency offsets that determine the spatial location of the centre of the slice, it is important to determine the thickness of a slice $\Delta z$ to achieve sufficiently intense signals while simultaneously avoiding an overlap of slices. $\Delta z$ is given by equation (1-2):

$$
\Delta z=\frac{2 \pi \cdot \Delta v_{\mathrm{BW}}}{\gamma G_{\mathrm{z}}}
$$

$\Delta v_{\mathrm{BW}}$ is the excitation bandwidth of the soft pulse which depends on the individual pulse shape and pulse duration. This also illustrates that a hard pulse is not appropriate as it exhibits a too wide excitation bandwidth for selective excitation (at standard gradient strengths). Usually, simulation protocols as included in NMR software suites (e.g. nmrsim or stdisp in Bruker Topspin) are applied to calculate these excitation bandwidths.

\subsubsection{Applications}

Magnetic resonance imaging (MRI) as known from medicinal diagnostics and research resembles the most prominent application of spatially resolved NMR spectroscopy. ${ }^{[39]}$ While the technical details of modern MRI get increasingly complex and require elaborate theoretical treatment, its basic principle can be transferred from SSE. The spatial selection of slices is extended by a phase and frequency encoding gradient that enables 3D imaging (Figure 1-6).

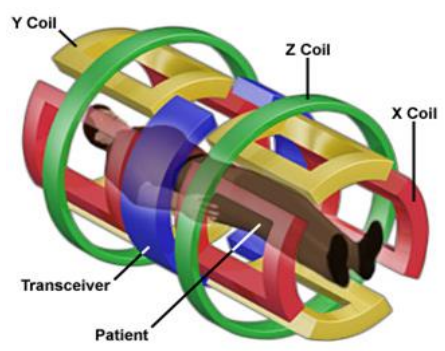

Figure 1-6: Schematic setup of an MRI scanner with a transmitter/receiver (transceiver) unit and three magnet coils $(x, y$ and $z$ ) that encode frequencies in 3D space. Reprint from reference [40]. Copyright 2015 National High Magnetic Field Laboratory. 
Note that magnetic information as acquired from MRI is usually restricted to relaxation rates and signal intensities of the $\mathrm{H}_{2} \mathrm{O}$ resonance. ${ }^{[39]}$ In contrast, liquid state NMR spectroscopy allows slice-selection combined with the acquisition of high resolution data that includes information like the chemical shift and $J$-couplings. In recent years, this approach led to a number of surprisingly diverse experiments in which SSE has been utilized to

i) reduce recycle delays and accelerate acquisition,

ii) achieve homo-decoupling,

iii) investigate heterogeneous samples along their spatial dimensions.

\section{i) Reduction of recycle delays and acceleration of acquisition}

Wagner et al. have shown that a fast reaction can be observed without inter-scan delays by consecutively exciting nuclei in different slices. ${ }^{[41]}$ This means that after successive acquisitions of signals (no deadtime) from different slices, full longitudinal magnetization of the initial slices has usually been reached again and the scans can be directly repeated to ensure an ample observation of the reaction.

This method is closely related to the reduction of recycle delays in order to decrease the overall acquisition time of $2 \mathrm{D}$ experiments as has been done in correlation spectroscopy (COSY) or ${ }^{[42]}$ heteronuclear multi quantum coherence (HMQC) ${ }^{[43]}$ spectroscopy. Furthermore, especially Frydman has developed elaborate pulse sequences in which $\mathrm{t} 1$ increments are detected along the spatial dimensions of the sample in one instance to allow single-shot (ultra-rapid) 2D spectroscopy such as acquisition of a COSY spectrum in $0.22 \mathrm{s.}^{[44]}$

\section{ii) Homo-decoupling}

Very recently, the suppression of proton-proton couplings has been gaining importance in NMR based research. ${ }^{[45]}$ In crowded spectra of mixtures ${ }^{[46]}$ or biomolecules homo-decoupling converts overlapping multiplets into distinct interpretable signals (Figure 1-7). ${ }^{[4]}$

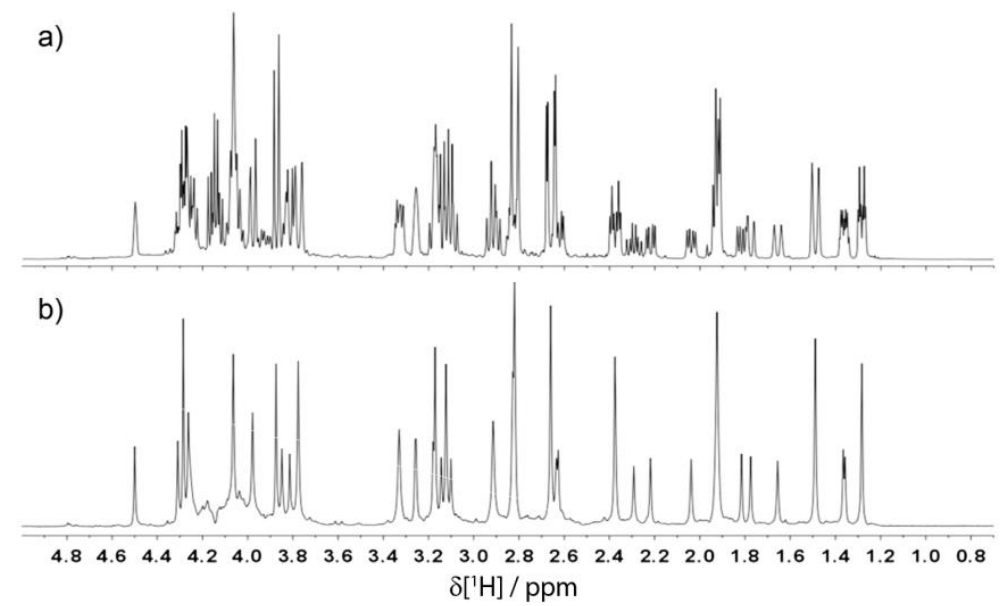

Figure 1-7: A regular a) and slice-selectively homo-decoupled spectrum b) of a mixture of strychnine and its degradation product. Reprint with permission from reference [47a]. Copyright 2015 Elsevier B. V.

Hence, so-called pure shift NMR spectroscopy virtually circumvents the resolution restriction of NMR which is otherwise only achieved by increasing the (expensive) magnetic strength of NMR 
spectrometers. ${ }^{[48]}$ Homo-decoupling can be achieved through several types of experiments as detailed elsewhere. ${ }^{[4]}$ One approach that depends on slice-selection has been pointed out by Zangger and Sterk ${ }^{[49]}$, picked up by the research community and driven further developments. Nowadays, homodecoupling has been combined with frequently used (pseudo) 2D techniques which in turn significantly profit from the improved resolution. Popular examples are pure shift DOSY ${ }^{[50]}$, pure shift heteronuclear single quantum coherence HSQC ${ }^{[51]}$, pure shift $\operatorname{COSY}^{[52]}$ and pure shift nuclear Overhauser enhanced spectroscopy (NOESY) ${ }^{[52]}$.

\section{iii) Investigation of heterogeneous samples along their spatial dimensions}

The third kind of SSE-based experiments outlined here is closely related to MRI. Within heterogeneous samples slices are excited to correlate chemical information with spatial resolution. In similar studies Pöppler et al. ${ }^{[37]}$ and Trigo-Mouriño et al. ${ }^{[53]}$ observed the distribution of small molecules within crosslinked polymer gel samples (Figure 1-8).
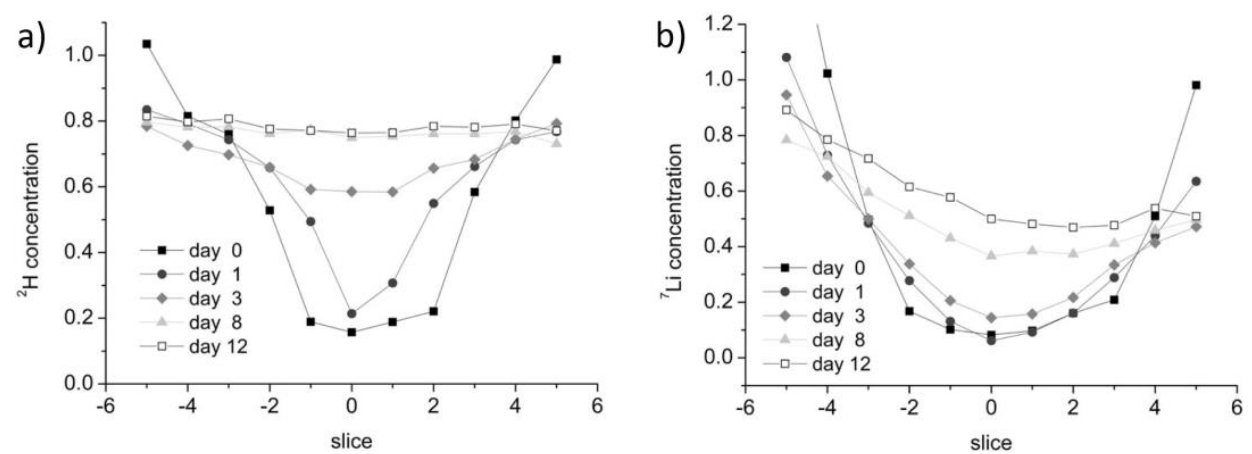

Figure 1-8: ${ }^{2} \mathrm{H}$ (a) and ${ }^{7} \mathrm{Li}$ (b) concentration within swollen polystyrene polymer sticks after various days of immersion in solutions of lithiumhexamethyldisilazane in toluene- $d_{8}$. Concentrations were determined from signal intensities in SSE spectra. Reprint with permission from reference [37]. Copyright 2013 Wiley VCH.

Pöppler et al. were able to describe the swelling behaviour and homogeneity of a polystyrene (PS) gel, based on its uptake of solvent (toluene- $d_{8}$ ) and solute (lithium hexamethyldisilazane). Both compounds were characterized by ${ }^{2} \mathrm{H}$ (Figure 1-8, a) and ${ }^{7} \mathrm{Li}$ signals (Figure 1-8, b) of sample slices over the course of several days. Trigo-Mouriño et al. observed $\mathrm{CDCl}_{3}$ in polymethylmethacrylate and emphasized the implications of the solvent signal splitting on the shimming procedure. ${ }^{[33]}$

Other experiments aimed at the description of biphasic systems via SSE. This led to a remarkable study in which the distribution coefficients of pharmaceutical ingredients (e.g. ibuprofen and paracetamol) in biphasic octanol-water $(\mathrm{pH}=7.4)$ samples were determined and found to be coherent with results from classical time consuming methods like the so-called shake-flask technique. ${ }^{[54]}$ Hence, this application can be readily transferred to pharmaceutical screening processes since distribution coefficients resemble prevalent parameters in toxicity studies. ${ }^{[55]}$

It is worth noting that by decreasing the excited volume, magnetic field imperfections along $z$ are compensated. ${ }^{[56]}$ Hence, SSE is naturally a most convenient technique the examination of heterogeneous samples which exhibit particularly strong inhomogeneity. ${ }^{[57]}$ 


\subsection{Anisotropic NMR Spectroscopy}

NOESY ${ }^{[58]}$ and $J$-coupling analysis ${ }^{[59]}$ are well established techniques for 3D structure determination. These methods allow the detection of through-space connectivity and provide vital angular information, respectively. Nevertheless, several disadvantages such as the low limit on distances $r$ between spins for the detection of NOE contacts $\left(\sim 1 / r^{6}\right)$ and the dependency on well-connected spin-systems for $J$ couplings increase the demand for complementary techniques.

In modern solid state NMR spectroscopy, dipolar ${ }^{[60]}$ and quadrupolar couplings ${ }^{[61]}$ supply additional, rich information on the $3 \mathrm{D}$ structure of molecules. This information is, at first sight, lost in isotropic liquid state NMR spectroscopy for the benefit of high resolution. ${ }^{a}$ However, in 1963, Saupe et al. showed that dipolar as well as quadrupolar couplings can be residually recovered by partially aligning molecules in so-called uniaxial environments such as solutions of nematic liquid crystals (Figure 1-9). ${ }^{[63]}$

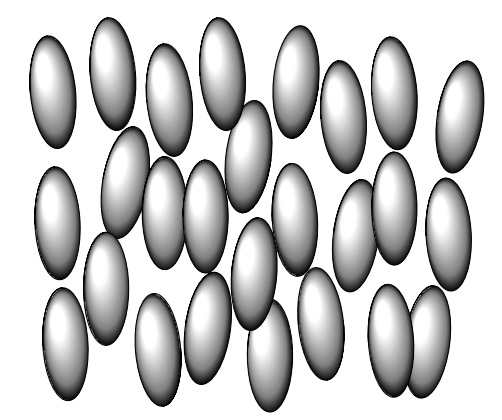

Figure 1-9: Schematic representation of molecules constituting a nematic liquid crystal phase. Note that a net orientation of the long molecular axes induces an alignment of potential solutes (framed within the blank space) through hindered rotation.

\subsubsection{Residual Dipolar Couplings}

\section{Theoretical Basics of (Residual) Dipolar Couplings}

The dipolar coupling $D$ between two adjacent nuclei T and S with spin $I=1 / 2$ can be described through equation (1-3):

$$
D=-\frac{3}{8 \pi^{2} R^{3}} \gamma_{\mathrm{T}} \gamma_{\mathrm{S}} \mu_{0} \hbar\left(\cos ^{2} \theta-\frac{1}{3}\right)
$$

$R$ is the distance between nuclei $\mathrm{T}$ and $\mathrm{S}, \gamma_{\mathrm{T}}$ and $\gamma_{\mathrm{S}}$ are the gyromagnetic ratios of nuclei $\mathrm{T}$ and $\mathrm{S}$, respectively, $\mu_{0}$ is the (magnetic) vacuum permeability, $\hbar$ is the Planck's constant divided by $2 \pi$ and $\theta$ is the angle between the magnetic field vector $\vec{B}_{0}$ and the vector connecting $S$ and $T$ (Figure 1-10). ${ }^{[64]}$

a Indeed, long range dipolar couplings indirectly become visible in solution as well, since dipolar couplings cause the NOE effect. ${ }^{[62]}$ 


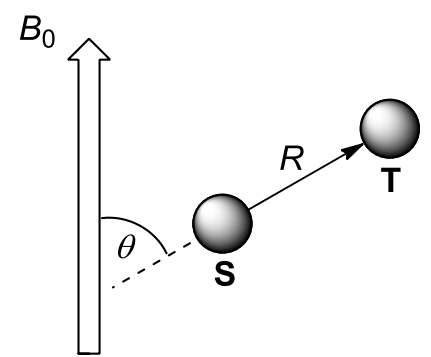

Figure 1-10: The angle $\theta$ is defined by the internuclear vector between $S$ and $T$ with length $R$ and the vector for the magnetic field $\vec{B}_{0}$.

Note that $D$ becomes strongest at an angle of $0^{\circ}$, which is similar to bar magnets that adopt a head to tail configuration as the lowest energy configuration (Figure 1-11).

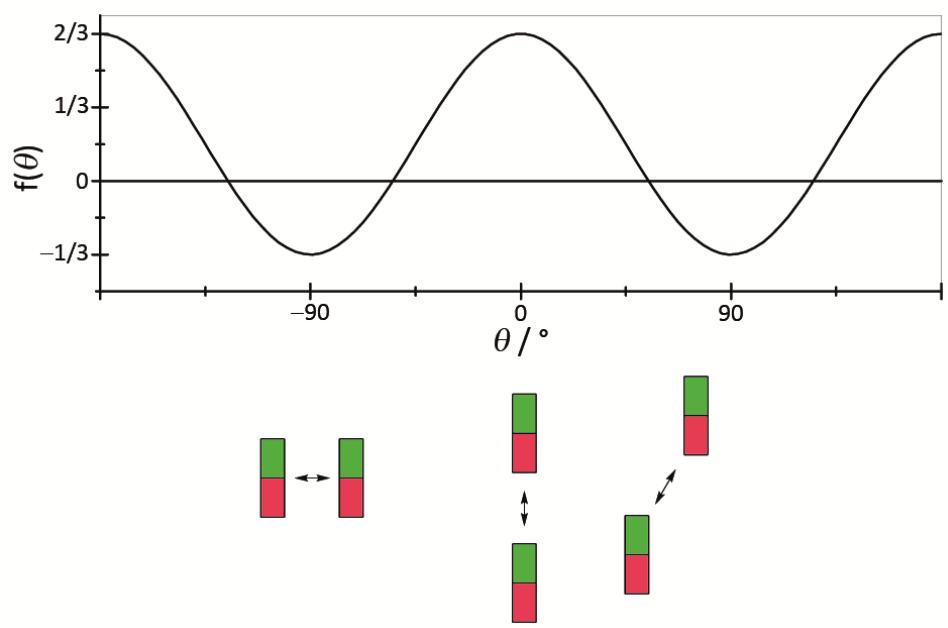

Figure 1-11: The angle term $f(\theta)=\cos ^{2}(\theta)-1 / 3$ imposes the limits for the strength of the magnetic dipolar coupling. This is similar to the interaction of bar magnets that show the strongest repulsive interaction at $90^{\circ}$ (left) and strongest attractive interaction at $0^{\circ}$ (middle). Note that the value of the magic angle (right), characterized by the vanishing interaction, depends on the distance and spatial dimensions of the bar magnets. For nuclei in molecules and their magnetic moments the magic angle is at $54.7^{\circ}$.

In contrast, $D$ vanishes to give one sharp signal if $\theta$ is at an angle of $54.7^{\circ}$ which is called the magic angle. A molecule in solution to uniformly tumbles around the Cartesian $x, y$, and $z$ axes. The averaged composite tumbling of the molecule is then around a room diagonal of a cube which is at $54.7^{\circ}$ which in turn leads to the vanishing dipolar coupling.

Dipolar couplings have long been in the focus of solid state NMR spectroscopy and the so-called Pake pattern signal of a powdered sample (Figure 1-12) is a prominent trait within a solid state spectrum. ${ }^{[60]}$ 


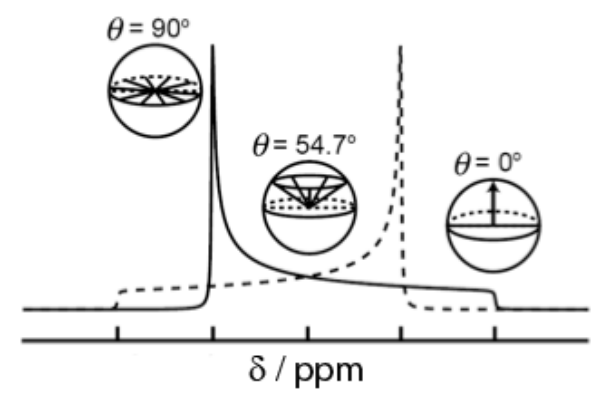

Figure 1-12: Typical Pake pattern of a powdered sample containing nuclei with $I=1 / 2$. Orientation with $\theta=0^{\circ}$ is less probable than $\theta=90^{\circ}$. This corresponds to the minimum intensity ("feet") and the maximum of the signal, respectively.

In a powder $\theta$ can attain all values from $0^{\circ}$ to $90^{\circ}$ but the exact orientation along the magnetic field is statistically less probable (only one exact parallel and one antiparallel orientation) than the perpendicular orientation (all angles between $0^{\circ}$ and $360^{\circ}$ within the perpendicular plane). Therefore $\theta=0^{\circ}$ and $\theta=90^{\circ}$ correspond to the feet and the maximum of the Pake pattern, respectively. The coupling strengths between these features enable the direct determination of the distance between both spins according to equation (1-3).

While a powder exhibits a homogeneous distribution of orientations, uniaxial environments induce physical barriers that lead to an averaged, small, net orientation of (non-spherical) molecules. Hence, the orientation of molecules in these anisotropic media has to be expressed through a $3 \mathrm{D}$ probability space which is known as the alignment tensor $\mathbf{A}(1-4)$.

$$
\mathbf{A}=\left(\begin{array}{lll}
A_{\mathrm{xx}} & A_{\mathrm{xy}} & A_{\mathrm{xz}} \\
A_{\mathrm{yx}} & A_{\mathrm{yy}} & A_{\mathrm{yz}} \\
A_{\mathrm{zx}} & A_{\mathrm{zy}} & A_{\mathrm{zz}}
\end{array}\right)
$$

A is a second rank, traceless $\left(A_{\mathrm{xx}}+A_{\mathrm{yy}}=-A_{\mathrm{zz}}\right)$, symmetric $\left(A_{\mathrm{ij}}=A_{\mathrm{ji}}\right)$ tensor and therefore fully described through five independent matrix elements. $\mathbf{A}$ is closely related to the probability tensor $\mathbf{P}$ via equation (1-5):

$$
\mathbf{A}=\mathbf{P}-\frac{1}{3} \mathbf{1}
$$

Here, $\mathbf{1}$ is the identity matrix. In the principal axis system (PAS) of $\mathbf{P}$ (i.e. the diagonalized $\mathbf{P}_{\mathrm{PAS}}$, see equation (1-6)) the principal values $P_{\tilde{\mathrm{x}}}, P_{\widetilde{\mathrm{y}}}$ and $P_{\tilde{\mathrm{z}}}$ correspond to the physical probabilities to find the magnetic field vector $\vec{B}_{0}$ along the associated axis $x, y$ and $z$, respectively.

$$
\mathbf{P}_{\mathrm{PAS}}=\left(\begin{array}{ccc}
P_{\tilde{\mathrm{x}}} & 0 & 0 \\
0 & P_{\widetilde{\mathrm{y}}} & 0 \\
0 & 0 & P_{\tilde{\mathrm{z}}}
\end{array}\right)
$$

Note that these preferences for certain orientations are usually very weak, hence, values for $A_{\mathrm{i}}$ within typical modern alignment media (vide infra) are in the orders of $10^{-5}$ to $10^{-4}$. This leads to so-called residual dipolar couplings (RDCs) in the order of several $\mathrm{Hz}$ as can be obtained by equation (1-7). 


$$
\bar{D}=-\frac{3}{8 \pi^{2} R^{3}} \gamma_{\mathrm{T}} \gamma_{\mathrm{S}} \mu_{0} \hbar\left(\vec{r}^{T} \mathbf{A} \vec{r}\right)
$$

Here, the angular term of equation (1-3) is substituted by the product of the alignment tensor $\mathbf{A}$ and the unit vector $\vec{r}$ that characterizes the orientation between $\mathrm{S}$ and T and its transposed $\vec{r}^{T}$. Equation (1-7) is the most fundamental expression from which

i) RDCs can be (back-)calculated for a known alignment tensor

ii) the alignment tensor can be derived from a set of known experimental RDCs for a hypothetical structure. ${ }^{\mathrm{a}}$

Note that the bar above $D$ which signals the averaging of the dipolar coupling to an RDC in anisotropic media is omitted in the subsequent discussion.

\section{Experimental Determination of RDCs}

Most commonly, RDCs between directly bound $\mathrm{C}$ and $\mathrm{H}\left({ }^{1} D_{\mathrm{CH}}\right)$ are measured. Furthermore, $\mathrm{N}$ and $\mathrm{H}$ couplings $\left({ }^{1} D_{\mathrm{NH}}\right)$ are frequently observed in the backbone of protein structures. ${ }^{[6]}$ These one-bond RDCs can be collected by NMR spectroscopic methods in which $\mathrm{C}-\mathrm{H}(\mathrm{N}-\mathrm{H}$ etc.) couplings are either recorded in the direct (F2) or indirect (F1) dimension (Figure 1-13). ${ }^{[6]}$
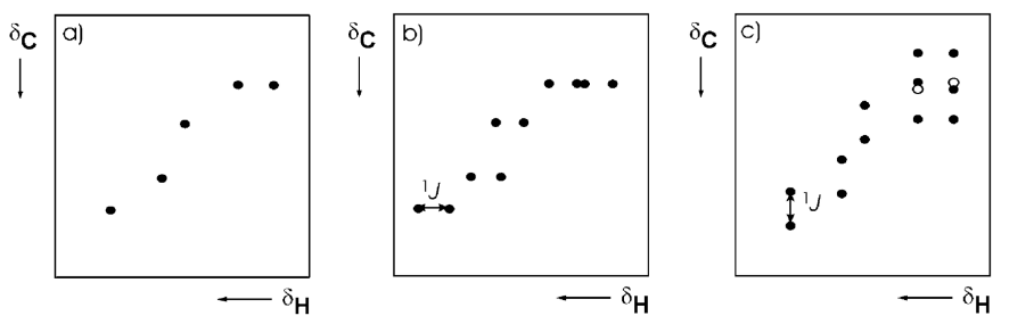

Figure 1-13: Schematic illustration of spectra from HSQC type experiments. a) Decoupled HSQC. b) HSQC coupled in direct (F2) dimension. c) HSQC coupled in indirect (F1) dimension. Reprint with permission from reference [66]. Copyright 2008 Wiley VCH.

The observed coupling (signal splitting) ${ }^{1} T_{\mathrm{XH}}(\mathrm{X}=\mathrm{C}, \mathrm{N})$ in anisotropic media is always a sum of the scalar ${ }^{1} J_{\mathrm{XH}}$ and dipolar ${ }^{1} D_{\mathrm{XH}}$ coupling as both inseparably develop in the course of the experiment.

The spectrum of an isotropic sample yields only scalar couplings such as ${ }^{1} J_{\mathrm{CH}}$ (Figure 1-13 a) and it is important to emphasize that the sign of this J-coupling cannot be directly obtained from the HSQC experiment. However, one-bond $J$-couplings between nuclei that exhibit the same sign for the gyromagnetic ratio are known to be always positive. ${ }^{[67]}$ This is the case for ${ }^{1} J_{\mathrm{CH}}$. The anisotropic sample yields the combined coupling (Figure 1-13 a and b). ${ }^{\mathrm{b}}$

\footnotetext{
${ }^{a}$ This corresponds to setting up an equation system from (1-3), with experimental RDCs and the vectors describing $\mathrm{C}-\mathrm{H}$ bonds in the proposed structure. The latter are then multiplied with the (five) unknown tensor elements of $\mathbf{A}$. Hence, five linearly independent equations can be used to determine the alignment tensor components.

${ }^{\mathrm{b}}$ Note that further definitions can be found in the literature with ${ }^{1} T_{\mathrm{CH}}={ }^{1} J_{\mathrm{CH}}+2 \cdot{ }^{1} D_{\mathrm{CH}} \cdot{ }^{[66]}$ Accordingly, equation (17) then contains an additional factor $1 \frac{1}{2}$. However, from an applied point, this is of minor importance since only ratios of RDCs are employed for further structure analysis.
} 


$$
{ }^{1} T_{\mathrm{CH}}={ }^{1} J_{\mathrm{CH}}+{ }^{1} D_{\mathrm{CH}}
$$

Simple subtraction $\left({ }^{1} T_{\mathrm{CH}}-{ }^{1} J_{\mathrm{CH}}\right)$ yields the RDC ${ }^{1} D_{\mathrm{CH}}$ with the correct sign (as long as ${ }^{1} D_{\mathrm{CH}}<{ }^{1} J_{\mathrm{CH}}$ since only the magnitude of ${ }^{1} T_{\mathrm{CH}}$ is measured. Acquisition of spectra in $\mathrm{F} 1$ and $\mathrm{F} 2$ has individual benefits and drawbacks: Most prominently, F2-resolved couplings offer good resolution at short experimental times. However, as remarked above, typical ${ }^{\mathrm{n}} \mathrm{X}_{\mathrm{XH}}$ scalar couplings (usually between protons) ${ }^{\mathrm{a}}$ evolve during acquisition as well and may lead to broad signals that complicate the extraction of the dipolar couplings (unless no additional homo-decoupling is applied). In contrast, F1-coupled spectra only exhibit sharp signals in the corresponding dimension since virtually no two ${ }^{13} \mathrm{C}$ nuclei are neighboured/scalar coupled at natural abundance. A significant drawback of F1-coupled acquisition is the aggravated extraction of couplings between $\mathrm{C}$ and diastereotopic protons which is hinted in (Figure 1-13 c). Small differences in RDCs lead to an overlap and unreliable values.

One of the most prominent types of experiments to achieve F2 coupled spectra is the clean-inphase (CLIP) HSQC experiment which has been developed by Luy et al. (Figure 1-14). ${ }^{[68]}$

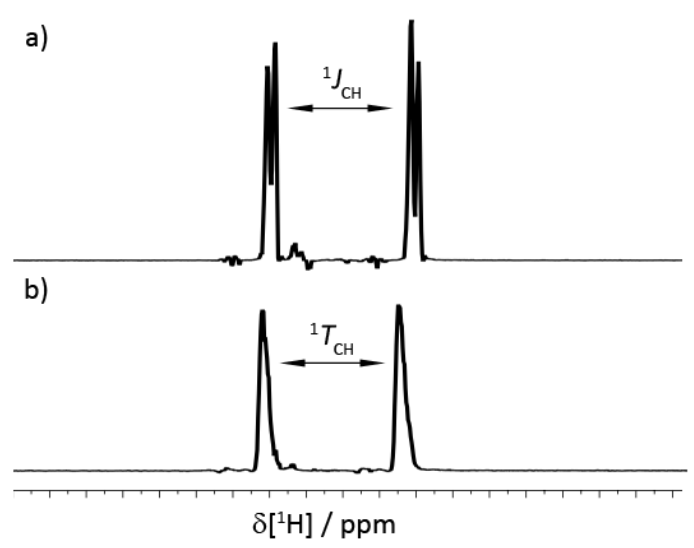

Figure 1-14: Exemplary slices of two CLIP-HSQC spectra for $\mathrm{C} 2-\mathrm{H} 2$ couplings (ortho $\mathrm{H}$ and carbon) of toluene molecules. a) Isotropic ${ }^{1} J_{\mathrm{CH}}$ coupling in neat dichloromethane. b) Anisotropic ${ }^{1} T_{\mathrm{CH}}$ coupling in crosslinked PS immersed in dichloromethane. Note that the change in chemical shift results predominantly from changes of the chemical environment/susceptibility.

Further experiments to extract long range dipolar couplings ${ }^{\mathrm{n}} D_{\mathrm{CH}}$ and ${ }^{\mathrm{n}} D_{\mathrm{HH}}$ may add valuable information but require a more elaborate treatment, since they are considerably weaker and the sign of the scalar coupling constant has to be determined. ${ }^{[69]}$

\section{Applications of Residual Dipolar Couplings}

Until today, RDCs have been increasingly employed in the study of large biomolecules ${ }^{[0]}$ as well as small- to medium-sized synthetic and naturally occurring substances. ${ }^{[66,71]}$ Frequent applications include the analysis of relative orientations between molecular fragment ${ }^{[72]}$, structure refinement of $\mathrm{XRD}_{\text {data }}{ }^{[73]}$, the analysis of (constrained) molecular dynamics data ${ }^{[74]}$ and structure verification ${ }^{[75]}$. The first three applications are almost exclusively encountered in biomolecular studies. The most

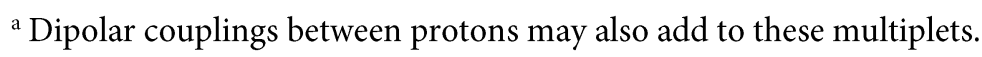


widespread application of RDCs in studies on small molecules is (3D) structure validation. After experimental acquisition of RDCs as described above, four basic steps constitute this procedure:

i) Generation of a comprehensive set of possible molecular structures.

ii) Determination of alignment tensors from RDCs and structural proposals.

iii) Back-calculation of RDCs from structural proposals and alignment tensors.

iv) Assessment of the validity of the structural proposal based on experimental and backcalculated RDCs.

In an interesting study Kummerlöwe et al. showed how the unknown structure of a sideproduct in an electrophilic cyclization of an azide-containing 1,5-enyne was determined via RDCs. ${ }^{[76]}$ With only little knowledge restricted to basic building blocks, the authors created a diverse set of 14 trial structures (Scheme 1-2).
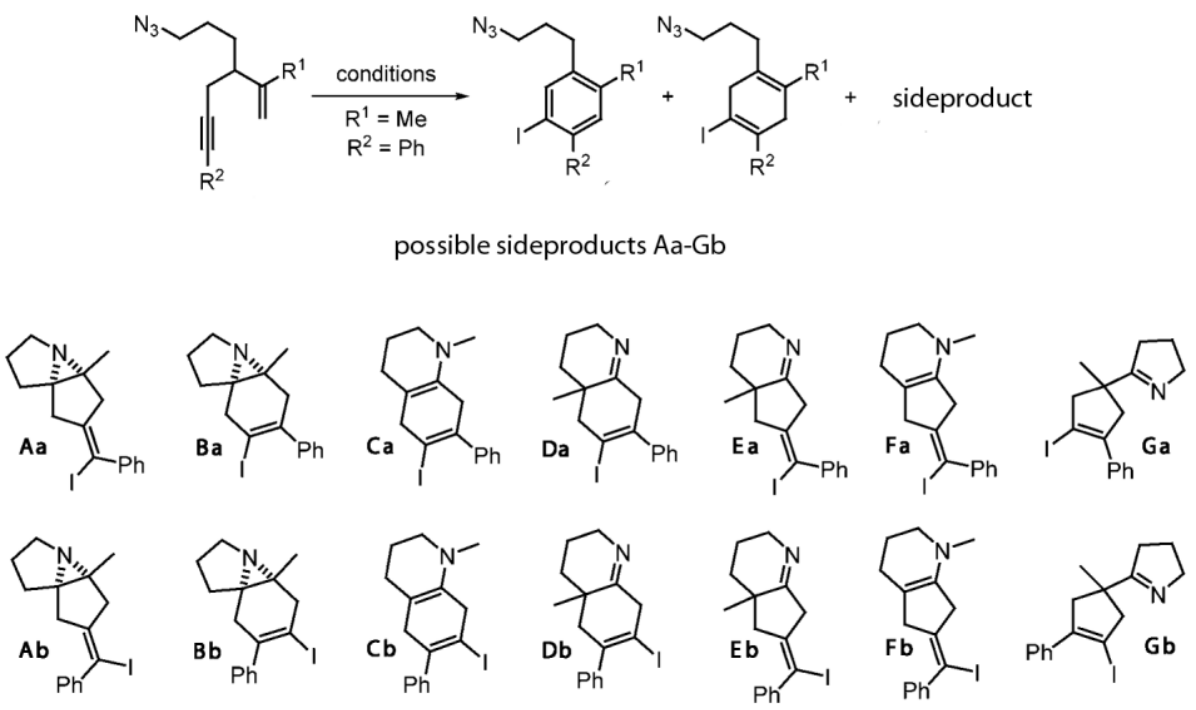

Scheme 1-2: Electrophilic cyclization reaction between an azide-containing 1,5-enyne leading to two main products and one unknown sideproduct. 14 possible structures for the sideproduct were created and verified/falsified through RDC analysis. Reprint with permission from reference [76]. Copyright 2011 Wiley VCH.

Next, the authors determined the alignment tensor A from these structural proposals and experimental RDCs $D_{\text {exp }}$ by setting up and solving linear equation systems from (1-7). In turn, the alignment tensor enabled back-calculation of RDCs ( $\left.D_{\text {calc }}\right)$. From the highest compliance between $D_{\text {exp }}$ and $D_{\text {calc }}$ Kummerlöwe et al. finally concluded that the reaction led to "the unexpected formation of the tricyclic compound" Ba (Scheme 1-2). This structure verification procedure including the setting up of appropriate equation systems is described in more detail in section 4.2.

Note that the simplest approach to utilize RDCs is to estimate relative orientations of prominent molecular features (e.g. helices in biomolecules) from comparison between suitable (parallel) RDCs. However, common RDC based studies heavily rely on the determination of the alignment tensor $\mathbf{A}$. This tensor can be either calculated from experimental RDCs and trial structures (as done by Kummerlöwe et al. and described in detail in section 4.2) or estimated from software relying on first principles such as the moments of inertia (Tramite ${ }^{[77]}$ or the molecular shape combined with simulated steric obstruction (PALES $)^{[78]}$. Both methods resemble very intuitive assessments on molecular 
orientation either by approximating that molecules in elongated pores preferably rotate around axes with small moments of inertia or that molecular rotation is hindered by a physical wall. Both approaches have been used in biomolecular studies. ${ }^{[79]}$ For small molecules benchmark molecular dynamics (MD) simulations have been successfully applied to examine their alignment in nematic liquid crystal solutions, but are so far not regularly employed in small molecules structure elucidation. ${ }^{[80]}$

However, the ever growing popularity and applicability of RDC based structure determination has enormously capitalized on the development of supportive and comprehensive software. PALES ${ }^{[78,81]}$ and REDCAT $^{[82]}$ for biomolecules and $\mathrm{MSpin}^{[83]}$ for small molecules today initialize RDC analysis implementation in routine NMR.

\subsubsection{Residual Quadrupolar Couplings}

\section{Theoretical Basics of (Residual) Quadrupolar Couplings}

Quadrupolar nuclei possess a nuclear spin with $I>1 / 2$ and feature non-spherical charge distributions. The resulting electric quadrupole moment $Q$ (usually denoted in the quantity $e Q$ where $e$ is the elementary charge) interacts with the electric surrounding (neighbouring atoms and electrons) that is characterized by the electric field gradient (EFG). The EFG can be fully described by a second-rank traceless, symmetric tensor $\mathbf{V}(1-9)$.

$$
\mathbf{V}=\left(\begin{array}{ccc}
V_{\mathrm{xx}} & V_{\mathrm{xy}} & V_{\mathrm{xz}} \\
V_{\mathrm{yx}} & V_{\mathrm{yy}} & V_{\mathrm{yz}} \\
V_{\mathrm{zx}} & V_{\mathrm{zy}} & V_{\mathrm{zz}}
\end{array}\right)
$$

In its principal axis system and with ordering $\left|V_{\tilde{\mathrm{x}}}\right| \leq\left|V_{\tilde{y}}\right| \leq\left|V_{\tilde{\mathrm{z}}}\right|$ the asymmetry parameter $\eta$ describes the cylindricity of the EFG (1-10):

$$
\eta=\frac{V_{\tilde{\mathrm{x}}}-V_{\widetilde{\mathrm{Y}}}}{V_{\tilde{\mathrm{z}}}}
$$

The quadrupolar nucleus may be illustrated by an axially symmetric ellipsoid localized within a negative electric charge distribution of surrounding electrons (Figure 1-15a). 
a)

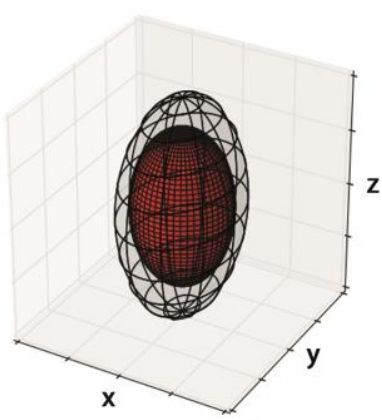

b)

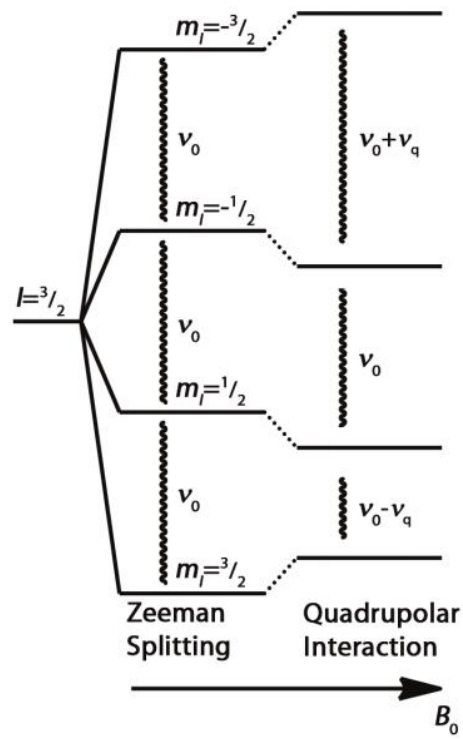

Figure 1-15: a) Schematic illustration of the quadrupolar nucleus (red) within the negative electric charge (colorless). b) The splitting of energy levels of a single nucleus with spin $I=3 / 2$ in an external magnetic field (Zeeman splitting) is further modified through the quadrupolar interaction.

The spin-states $m_{\mathrm{I}}$ of a quadrupolar nucleus in a magnetic field experience an energetic attenuation or amplification through interaction with the EFG (Figure 1-15 b). This results in $2 I$ spectroscopic transitions with $\Delta m_{\mathrm{I}}=1$. Hence, for a nucleus with $I=3 / 2$ three resonances can be observed: $v_{\mathrm{o}}$ is the central transition and $v_{0}+v_{\mathrm{q}}$ as well as $v_{0}-v_{\mathrm{q}}$ are the satellite transitions. According to this, the quadrupolar coupling $v_{\mathrm{q}}$ is the frequency difference between the central transition and either one of the satellite transitions. The following definition of the quadrupolar coupling and its orientation dependency is adopted from the conventions used by Man: ${ }^{[61 b] a}$

$$
v_{\mathrm{q}}=\frac{3 \chi}{4 I(2 I-1)}\left(3 \cos ^{2} \beta-1+\eta \sin ^{2} \beta \cos 2 \alpha\right)
$$

Here, $\chi$ is the quadrupolar coupling constant, $I$ is the nuclear spin, $\eta$ is the asymmetry parameter of the EFG and $\alpha$ and $\beta$ are the Euler angles of the magnetic field vector in the PAS of the EFG (for a detailed definition and discussion of these angles see reference [84]). The quadrupolar coupling constant $\chi$ is usually defined by equation (1-12).

$$
\chi=\frac{e^{2} q Q}{h}
$$

Here, $e Q$ is the nuclear electric quadrupole moment, $e q$ is the largest component of the EFG tensor $\left(V_{\tilde{z}}\right)$ and $h$ is the Planck's constant. Usually, $\chi$ and $\eta$ can be readily determined from solid state powder spectra as depicted in Figure 1-16.

\footnotetext{
${ }^{a}$ Note that further definitions can be found in the literature with an additional factor $1 / 2$. In these cases the satellite transitions in Figure 1-15 b) exhibit frequencies $v_{0} \pm 2 v_{\mathrm{q}}$.
} 


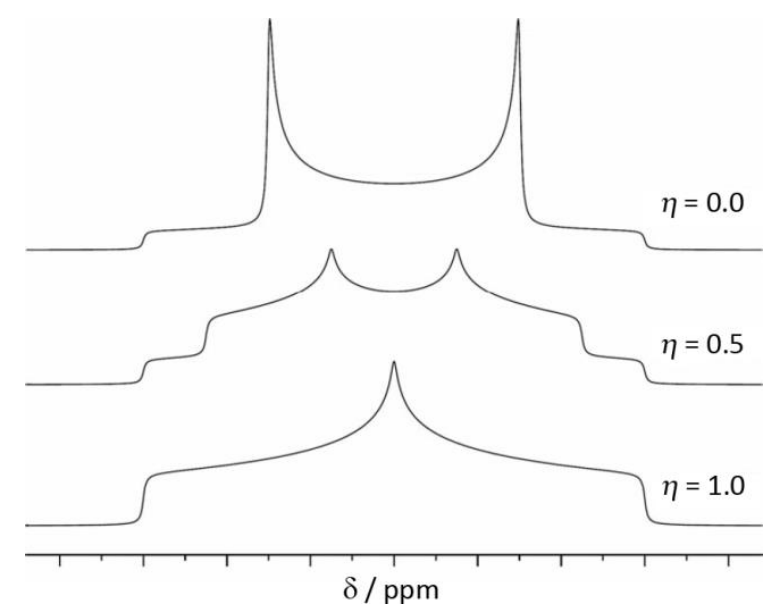

Figure 1-16: Typical powder pattern of a sample containing nuclei with $I=1$ for EFGs with increasing asymmetry parameter $\eta$. Reprint with permission from reference [61c]. Copyright 2013 Wiley VCH.

In the case of no asymmetry $(\eta=0)$ the EFG can be treated like a vector and is analogous to dipolar couplings. $\chi$ can then be directly read of the difference between the maxima of the peak or its feet. Increasing asymmetry is usually well determined from fitting procedures and comparison of frequency differences between feet and maxima.

In the case of partial alignment, no continuous distribution of orientations/frequencies is observed, but an averaged partial alignment from which a number of satellite resonances result according to the nuclear spin $I$. The EFG tensor elements contribute to these frequency shifts but are modulated by their probability of being aligned along the magnetic field vector. This relationship defines the RQC $v_{\mathrm{rq}}$ as given in equation (1-13):

$$
v_{\mathrm{rq}}=\frac{3 e Q}{2 I(2 I-1) h} \sum_{\mathrm{i}, \mathrm{j}=\mathrm{x}, \mathrm{y}, \mathrm{z}} V_{\mathrm{ij}} A_{\mathrm{ij}}
$$

The equation contains a set of constants already known from above. Additionally, eq, $\eta$ and the angular term as included in (1-11) are substituted by a sum over all Cartesian products $V_{\mathrm{ij}}$ and $A_{\mathrm{ij}}$ which are elements of the EFG and the alignment tensor, respectively. Note the distinction between this Cartesian definition of the subscripts $i$ and $j$ (i.e. a shared reference frame for both tensors such as a molecular fixed frame or either one of the PAS of the tensors) and the usual conventions for naming and ordering the PAS elements of both tensors. This may easily lead to confusion and misunderstandings but will be acknowledged specifically at the appropriate passages of this thesis. 


\section{Applications of (Residual) Quadrupolar Couplings}

The majority of studies on quadrupolar nuclei employs solid state NMR spectroscopy and subsequent lineshape analysis of e.g. satellite transitions. Suitable simulation software like Dmfit (QUASAR ${ }^{[85]}$ or SIMPSON ${ }^{[86]}$ allow straightforward determination of $e q(\chi)$ and $\eta$ (i.e. the EFG V) from fitting simulated to experimental spectra. Corresponding techniques and detailed applications have been extensively reviewed elsewhere. ${ }^{[61]}$ Notably, within the group of Günther various studies on ${ }^{7} \mathrm{Li}$-containing compounds have been conducted and dependencies between the quadrupolar coupling constant $\chi\left({ }^{7} \mathrm{Li}\right)$ and angles X-Li-X $(\mathrm{X}=\mathrm{C}, \mathrm{N})$ were derived (Figure 1-17). ${ }^{\left[{ }^{[8]}\right.}$

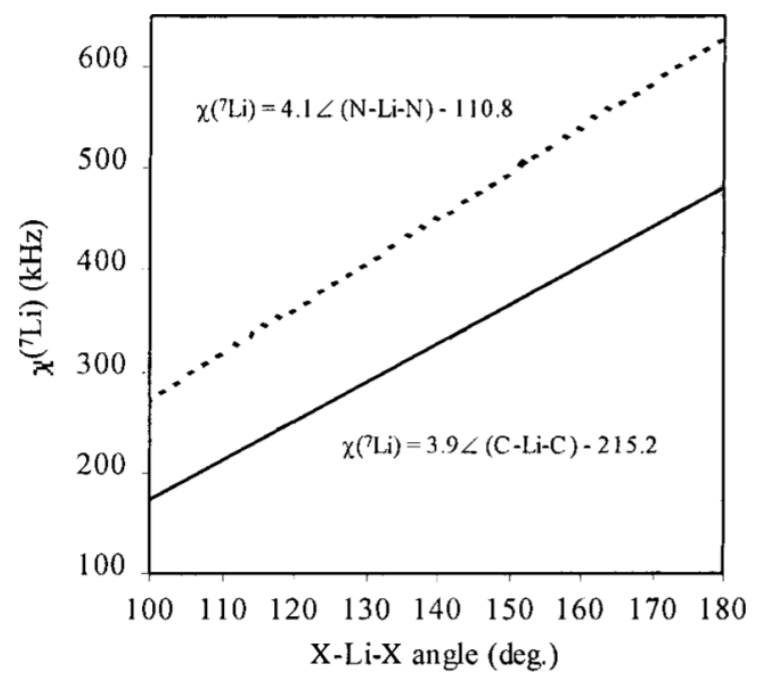

Figure 1-17: Correlation between the quadrupolar coupling constant $\chi\left({ }^{7} \mathrm{Li}\right)$ as determined from solid state NMR and the structural $\mathrm{N}-\mathrm{Li}-\mathrm{N}$ angle (dotted) or $\mathrm{C}-\mathrm{Li}-\mathrm{C}$ angle (solid) as determined from X-ray data. Reprinted with permission from reference [87b]. Copyright 2000 American Chemical Society.

These correlations may offer viable structural information for molecular compounds that do not crystallize. In contrast to RDCs, applications of RQCs are limited due to adverse atomic properties. In particular, strong quadrupolar moments induce fast relaxation and linebroadening and thereby prohibit the observation of RQCs in cases such as ${ }^{75} \mathrm{As}$ with $I=3 / 2$ and $Q=31.5 \mathrm{fm}^{2}$ which is about eight times higher than for ${ }^{7} \mathrm{Li}\left(Q=-4.01 \mathrm{fm}^{2}\right)$. Nevertheless, the applicability of RQCs in anisotropic solutions was recognized early on together with upcoming RDC techniques. Until today, especially the use of ${ }^{2} \mathrm{H}$ spectroscopy $\left(I=1 ; Q=0.286 \mathrm{fm}^{2}\right)$ has been attracting some attention since it can be employed for enantiomeric differentiation of deuterated solutes in chiral nematic liquid crystals. ${ }^{[88]}$ In these cases deuterons are assumed to experience a nearly axially symmetric EFG with $\left|V_{z z}\right|$ along the sigma bonds (similar to RDCs vide supra). Thus the RQCs are a direct and most precise measure of the molecular alignment which, in chiral alignment media, is dependent on the absolute configuration. Still, this usage is limited as it requires the challenging determination of RQC signs to concisely draw conclusions on absolute configuration. Recently, some progress to overcome this remaining challenge was made by Tzvetkova and Luy through the development of a quadrupolar exclusive COSY (Q.E.COSY) experiment. ${ }^{[89]}$ 
Besides these quantitative approaches Pöppler et al. ${ }^{[38]}$ showed that RQCs can be exploited in an innovative manner to distinguish between lithium aggregates. The authors persuasively reasoned that the values of RQCs $v_{\mathrm{rq}}$ as defined in equation (1-13) become zero either if $\mathbf{A}=0$, i.e. the aggregate is highly symmetric $\left(O_{\mathrm{h}}, T_{\mathrm{d}}\right.$ etc.) and no alignment within the gel is possible or if $\mathbf{V}=0$, i.e. the EFG around ${ }^{7} \mathrm{Li}$ is symmetric (Figure 1-18).

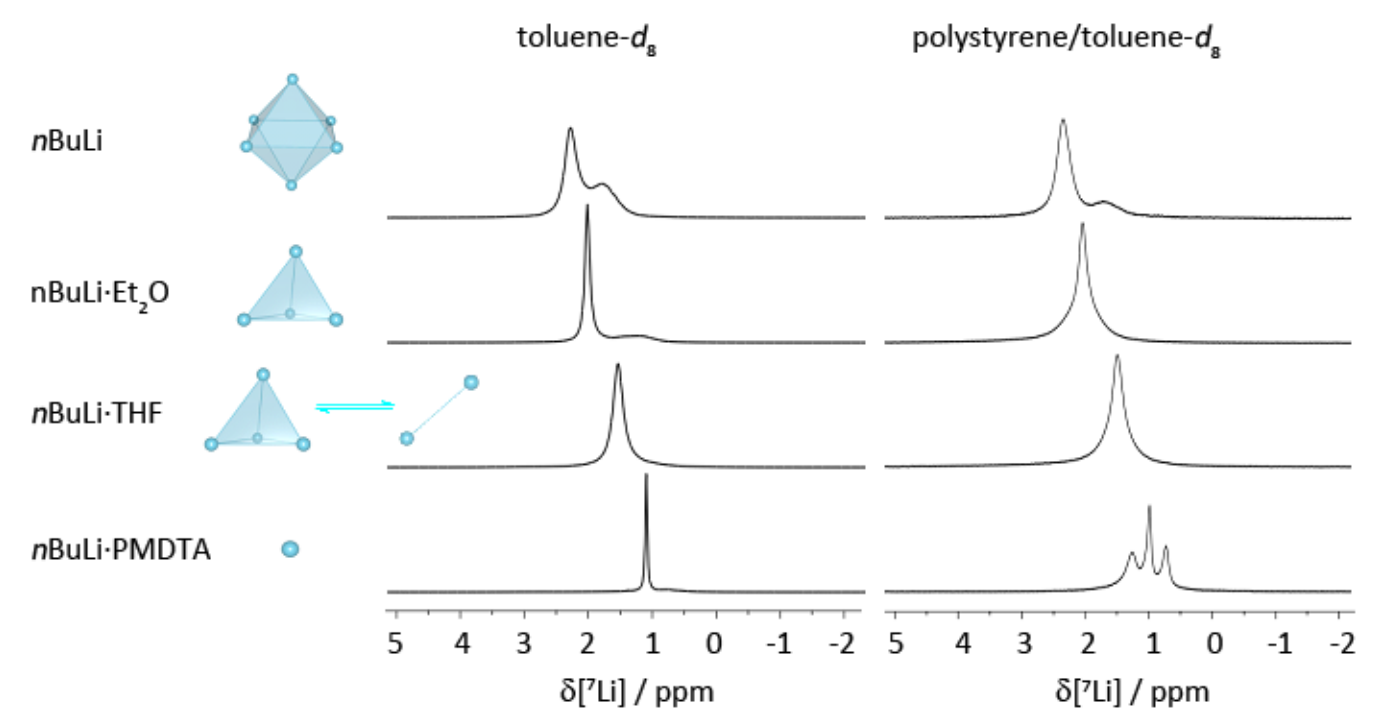

Figure 1-18: Aggregates of $n \mathrm{BuLi}$ and associated ${ }^{7} \mathrm{Li}$ spectra in isotropic (toluene- $d_{8}$ ) and anisotropic (PS/toluene$d_{8}$ ) solution. Reprinted with permission from reference [38]. Copyright 2012 Wiley VCH.

Hence, this qualitative approach represents a smart and straightforward method to distinguish symmetric from asymmetric lithium aggregates. Yet, a more comprehensive determination of the EFG for molecules with $\mathbf{A} \neq 0$ will amend the possibilities of solid state NMR spectroscopy and facilitate structure determination as shown by Günther et al. ${ }^{[87]}$ This concept is explored in section 4.3.

\subsubsection{Alignment Techniques}

In 1963 Saupe et al. used solutions of strong alignment media like p-azoxyansiole (PAA) or p-butoxybenzoic acid (PBBA) which led to the first observations of anisotropic interactions (Scheme 1-3). However, these strongly orienting liquid crystals proved impractical for the early utilization of residual couplings since the RDCs and RQCs were too large to be determined with standard liquid state NMR techniques. Hence, the increase of research into anisotropic NMR spectroscopy was only initiated in the mid-90s when weak alignment media such as mixtures of dimyristoyl phosphatidylcholine (DMPC) and dihexanoyl phospatidylcholine (DHPC) (Scheme 1-3) were first described. ${ }^{[0]}$ 

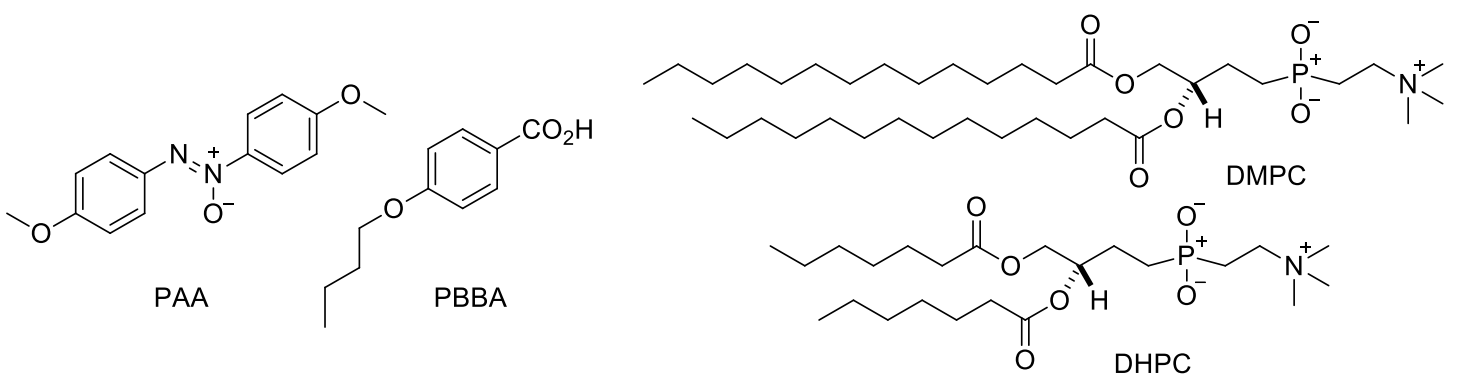

Scheme 1-3: Typical examples for molecular components of nematic liquid crystals: $p$-azoxyansiol (PAA), $p$ butoxy-benzoic acid (PBBA), dimyristoyl phosphatidylcholine (DMPC), dihexanoyl phospatidylcholine (DHPC).

Molecules oriented in these media showed RDCs in the order of $J$-couplings which could be experimentally determined from known NMR spectroscopic methods (vide supra). ${ }^{\left[{ }^{[0]}\right.}$ Alternatively, in 2000 Tycko et al. ${ }^{[70 a]}$ and Sass et al. ${ }^{[91]}$ independently revived an alignment concept already introduced by Deloche and Samulski in 1981: ${ }^{[92]}$ various crosslinked polymers can take up huge amounts of solvents to give polymeric gels with massively increased volume. Swelling of small cylindrical sticks that neatly fit into NMR tubes leads to an extension that is restricted to the orientation along the tube (also see Figure 1-19 b). Accordingly, this leads to polymer strands that are predominantly oriented along the direction of the swelling and thus yield elongated cavities within the gel. Molecules dispensed within the gel (cavities) exhibit degrees of alignment that can be easily adjusted by parameters such as gel type, crosslinking percentage or solvent. ${ }^{[93]}$ Hence, so-called strain induced alignment in a gel (SAG) initiated several studies from which a multitude of possible polymeric alignment media resulted (Table 1-1).

Table 1-1: Selected polymeric alignment media, appropriate solvents and special remarks.

\begin{tabular}{lll}
\hline & Solvents & Special remarks \\
\hline Polyacrylamide $^{[70 a, 91]}$ & Strongly polar (e.g. $\left.\mathrm{H}_{2} \mathrm{O}\right)$ & Seminal work \\
Gelatin $^{[94]}$ & Polar (e.g. dimethyl sulfoxide, & Chiral, can be made from gummibears \\
& $\left.\mathrm{H}_{2} \mathrm{O}\right)$ & \\
Polydimethylsiloxane $^{[95]}$ & Weakly polar (e.g. THF, & Single polymer resonance at 0.1 ppm \\
& $n$-hexane, benzene) & \\
Polystyrene $^{[93 \mathrm{a}]}$ & Weakly polar (e.g.THF, toluene) & Chemically inert \\
& & \\
\hline
\end{tabular}

Furthermore, applying external mechanical stress on polymer sticks is a convenient method to tune the alignment. Several stretching and compression devices have been developed that allow rapid preparation of samples. Note that uptake and sufficient equilibration of solvents in a gel may take several days to weeks. Application of outside forces reduces the time for sample preparation to a minimum. Figure 1-19 a) shows the components of a compression device as developed by Gayathri et al. in the group of Gil. 
a)

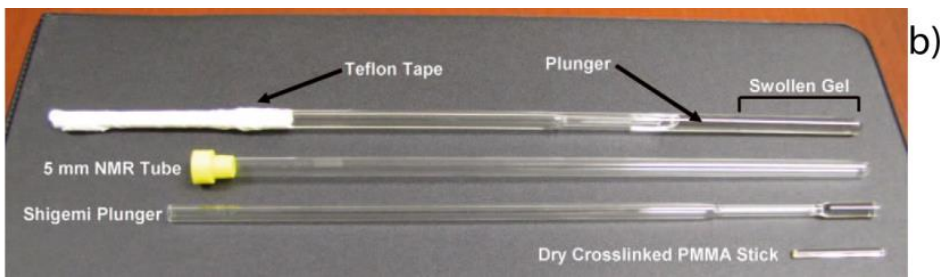

b)

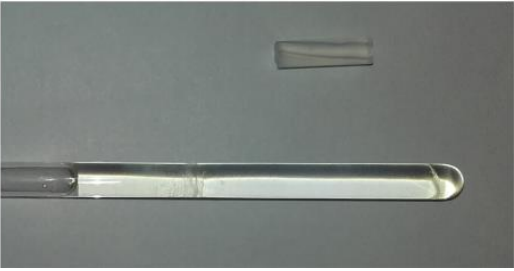

Figure 1-19: a) Compression device as applied by Gayathari et al. to mechanically achieve alignment within a gel. Reprint with permission from reference [37]. Copyright 2016 Wiley VCH. b) Swollen and non-swollen cylindrical PS.

The device is based on a Shigemi plunger that is pressed onto the polymer and thereby depletes the cavities within the gel, which in this case is a polymethyl methacrylate (PMMA) matrix. In Figure 1-19 b) non-swollen and confined, swollen cylindrical PS sticks can be visually compared.

Within the range of partial alignment media described above, suitable conditions can be found to study

i) $\quad$ RDCs between adjacent nuclei with spin $I=1 / 2$,

ii) Residual interactions between the electric quadrupole moment of a nucleus with spin $I>1 / 2$ and the surrounding EFG,

iii) changes in the observed chemical shift.

While RDCs and RQCs have been described in more detail in the sections above the residual chemical shift anisotropy (RCSA) is not in the focus of this thesis. Excellent reviews are found elsewhere. ${ }^{[96]}$ 


\section{OUTLINE}

Within the Stalke group the strong expertise in solid state structure determination of small metalorganic molecules ${ }^{[97]}$ has been increasingly complemented by liquid state NMR methods such as DOSY ${ }^{[34,36]}$ or NMR spectroscopy in anisotropic media. ${ }^{[38]}$ Comprehensive structure elucidation allows the derivation of structure-reactivity principles and in turn enables smart design of chemical reactions. The goal of this thesis is to further extend and improve the NMR toolbox. In particular, it aims at the utilization of SSE to observe dynamical processes and the application of anisotropic NMR spectroscopy to obtain ample structural information.

\section{Slice-Selective Excitation}

Horizontal sample slices can be excited through soft frequency-shifted pulses in the presence of a magnetic field gradient. Pöppler et al. have employed this technique to study the diffusion of small molecules into a PS gel matrix. ${ }^{[37]}$ In chapter 3 the SSE experiment lays the foundation for a variety of applications in which heterogeneous, dynamic systems can be observed to

i) characterize reaction intermediates,

ii) perform single-shot fast titrations,

iii) perform qualitative NMR chromatography.

The basic principles of these methods, as well as their implementation will be exemplified by tracing the reaction of $N, N, N^{\prime}, N^{\prime \prime}, N^{\prime \prime}$-pentamethyldiethylenetriamine (PMDTA) with $n \mathrm{BuLi}$, performing a titration of $\mathrm{Li}^{+}$with 12-crown-4 and achieving chromatographic separation of four prototypical amino acids.

\section{Anisotropic NMR Spectroscopy}

Crosslinked polymer matrices induce partial alignment of molecules in solution, which leads to additional nuclear spin interactions, namely RDCs and RQCs. These observables are increasingly employed in the determination of the configuration and conformation (i.e. the 3D structure) of small molecules. ${ }^{[66,70-71,88]}$ In chapter 4 possibilities are shown on how to utilize RDCs and RQCs to

i) observe the alignment of small molecules in polymers,

ii) determine the 3D structure of molecules,

iii) determine the aggregation of lithium organic compounds.

These applications are exemplified by the observation of small aromatic molecules oriented in PS and polybutylacrylate, the structure elucidation of synthetic $\beta$-amino acid esters containing an indane moiety and the determination of the aggregation of the lithium organic compound cyclopentadienyl lithium (CpLi). 


\section{Applications of SLice-Selective NMR Spectroscopy}

\subsection{Preparation of the SSE Experiments}

Although SSE is a robust and straightforward method, it requires an accurate technical preparation:

i) The determination of the magnetic field gradient strength.

ii) The setting of the pulse duration.

iii) The determination of the spatial detection sensitivity.

These steps have been adopted and advanced in the course of this work.

\section{Determination of the magnetic field gradient strength}

According to equation (1-1), for a given nucleus with gyromagnetic ratio $\gamma$ the position of the slice $z$ depends on the strength of the magnetic field gradient $G_{z}$ and the frequency offset $v_{z}$ of the soft-pulse.

$$
v_{\mathrm{z}}=\frac{\gamma \cdot G_{\mathrm{z}} \cdot z}{2 \pi}
$$

In the case of Bruker NMR probes the gradient strength $G_{\mathrm{z}}$ is adjusted by an electric current $I_{\mathrm{a}}$ that runs through the gradient coils. ${ }^{\mathrm{a}}$

$$
G_{\mathrm{z}}=g_{\mathrm{IG}} \cdot I_{\mathrm{a}}
$$

The corresponding proportionality constant $g_{\mathrm{IG}}$ is a factory-determined value that often lacks reliability, in which case frequency offsets lead to inaccurate assumptions about the positions of slices. This prohibits further quantitative analysis such as the spatial distribution of molecules. Hence, the gradient strength must be evaluated beforehand.

One common evaluation method is to reproduce literature-known diffusion coefficients through DOSY measurements which are based on magnetic field gradients. ${ }^{[30]}$ If DOSY experiments are run with sufficient care for experimental conditions like temperature, viscosity and concentration, only inaccurate gradient strengths can lead to values that deviate from literature. In turn, this deviation allows the estimation of a reliable value for $g_{\mathrm{IG} .}{ }^{[98]}$

An alternative technique that requires less expensive care for the experimental conditions, depends on the imaging of cylindrical discs ( ${ }^{1} \mathrm{H}$ NMR-silent solids such as perfluorinated polymers) with welldefined spatial dimensions. ${ }^{[99]}$ The longitudinal image of these cylindrical discs in the frequency domain is proportional to its spatial dimensions and $G_{z}$ is subsequently derived as the proportionality constant. A very similar approach was utilized in this work that further illustrates this technique:

A capillary filled with $\mathrm{H}_{2} \mathrm{O}$ (similar to the experimental setup known from the Evans method for the determination of susceptibility ${ }^{[100]}$ ) is immersed in an NMR tube filled with $\mathrm{D}_{2} \mathrm{O}$. The capillary is then

${ }^{\text {a }}$ Technical specifications depend on the probe, standard broadband observe (BBO) and inverse (BBI) probe allow $I_{\max }=10 \mathrm{~A}$. 
shifted between two positions $(\Delta z)$ at the top and the bottom of the active (excitable) volume of the NMR tube (Figure 3-1 a). At each step the exact position of the capillary is measured with a sliding calliper and subsequently a standard NMR imaging sequence (see 5 Experimental Part) consisting of a spin echo combined with two pulsed gradients (gradient strength at $20 \% I_{a, \max }$ ) is applied to obtain images as shown in Figure 3-1 b.

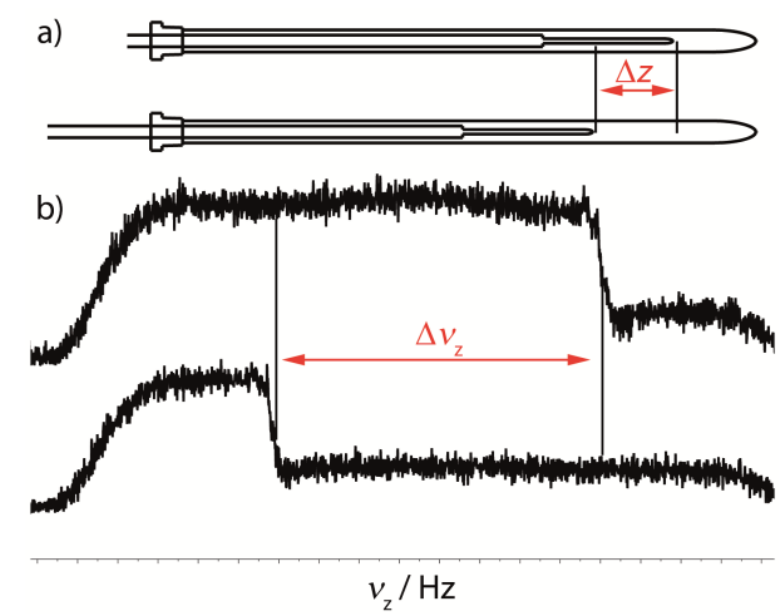

Figure 3-1: a) Two positions of the capillary within the NMR tube and their difference $\Delta z$. b) Corresponding images in the frequency domain with difference $\Delta v_{z}$.

The steep descent in the frequency domain corresponds to the bottom position of the capillary (more precisely, the definite $\mathrm{H}_{2} \mathrm{O}$ volume) and quantitative, reproducible values can be read off at half of the maximum signal intensity. Experimental differences between positions $\Delta z$ and frequencies $\Delta v_{z}$ are shown in Table 3-1.

Table 3-1: Differences between positions $\Delta z$ and frequencies $\Delta v_{z}$ from three repetitions of sliding the capillary within the NMR tube as illustrated in Figure 3-1.

\begin{tabular}{cccc}
\hline & Experiment 1 & Experiment 2 & Experiment 3 \\
\hline$\Delta z / \mathrm{mm}$ & 8.8030 & 8.5770 & 8.8830 \\
$\Delta v_{z} / \mathrm{Hz}$ & 38437 & 37321 & 38596 \\
\hline
\end{tabular}

These values allow straightforward calculation of the real gradient strength $G_{z}$ :

$$
G_{\mathrm{z}}=\frac{2 \pi \cdot \Delta v_{\mathrm{z}}}{\gamma \cdot \Delta z_{\mathrm{z}}}
$$

With a known $I_{a, \max }=10$ A this resulted in a spectrometer specific constant $g_{\mathrm{IG}}$ of $5.1 \pm 0.1 \mathrm{Gcm}^{-1} \mathrm{~A}^{-1}$, which considerably deviates from the value $\left(5.6 \mathrm{Gcm}^{-1} \mathrm{~A}^{-1}\right)$ initially stated in the hardware specification of the instrument. Note that for the newly determined $g_{\mathrm{IG}}$ and according to equation (1-1), at $I=0.5 I_{\max }$ a frequency offset $v_{z}$ of $98209 \mathrm{~Hz}$ excites a slice $9.0 \mathrm{~mm}$ above the center of the gradient coil. By applying the factory determined $g_{\mathrm{IG}}$ this slice would be assumed to be $8.2 \mathrm{~mm}$ above the centre of the gradient coil. This $9 \%$ difference clearly highlights the imperative to (re)determine the gradient strength for individual probes if they are used in slice-selective applications. 


\section{Setting of the pulse duration and power}

Excitation of an inhomogeneous sample that exhibits a continuous variation of properties along its spatial dimension (e.g. a shifting resonance that results from a concentration gradient along the NMR tube) leads to broadened signals. A less selective (harder) pulse excites a thicker slice which gives increased sensitivity but a further broadening due to the summation of the signals originating from different parts of the sample (Figure 3-2).

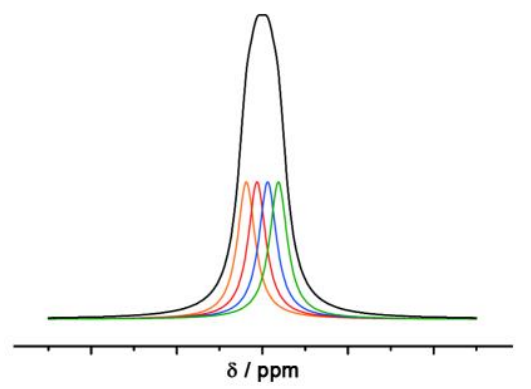

Figure 3-2: Schematic representation of signal widths in inhomogeneous samples. A changing chemical shift can be resolved by four selective excitations (colored) in the presence of a gradient. An unselective or less selective excitation leads to a summed, broadened signal (black).

As defined in equation (1-2), the signal thickness depends on the excitation bandwidth $\Delta v_{\mathrm{BW}}$ which in turn is governed in a non-trivial manner by the pulse duration and the shape of the pulse. The Bruker spectrometer software TopSpin includes a decent simulation tool (shape tool, stdisp) in which Fourier transformations of specified pulses yield their excitation bandwidths. In this work, exclusively Gaussian Cascade (G4) soft-pulses were applied. ${ }^{[101]}$ This shape achieves a nearly rectangular excitation profile across the excitation bandwidth of the pulse (Figure 3-3).

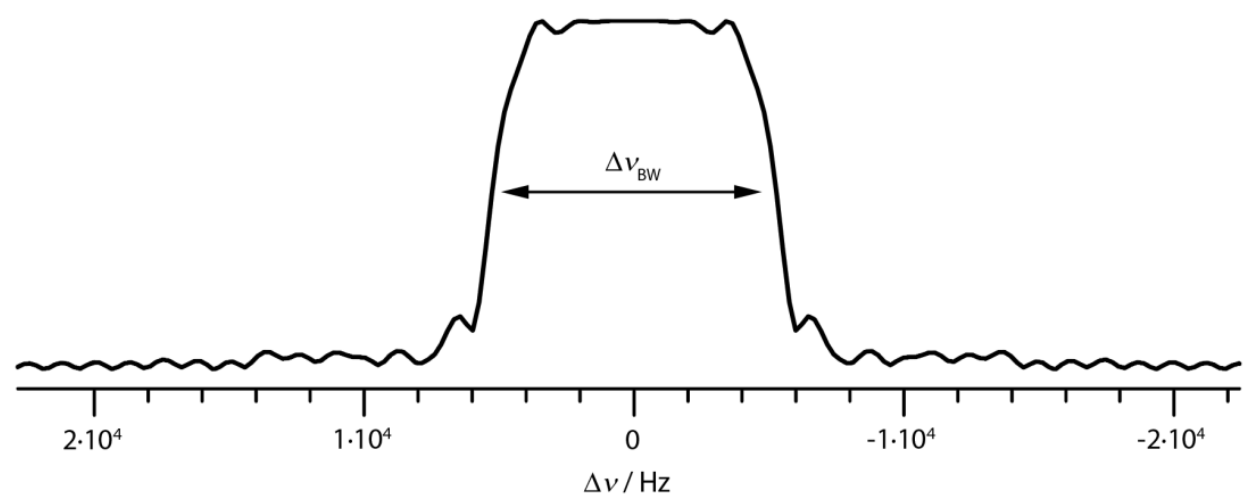

Figure 3-3: Excitation profile of an $800 \mu \mathrm{s}$ G4 Gaussian Cascade pulse with $90^{\circ}$ flip angle. The profile corresponds to a frequency bandwidth $\Delta v_{\mathrm{BW}}$ of $\sim 11 \mathrm{kHz}$ at half maximum intensity.

Furthermore, slices were chosen to be excited succinctly, i.e. they should be separated by $1 \mathrm{~mm}$ and exhibit an approximate thickness of $1 \mathrm{~mm}$ as well to avoid overlap of slices. The necessary parameters to obtain this mode are detailed in Table 3-2. 
Table 3-2: Pulse shape and pulse duration for nuclei relevant to this work that yield excitation bandwidths $\Delta v_{\mathrm{BW}}$ which at a gradient strength $G_{z}$ excite the desired slice thickness.

\begin{tabular}{cccccc}
\hline Nucleus & Pulse shape & $\begin{array}{c}\text { Gradient strength } \\
G_{z} / \mathrm{G} \cdot \mathrm{cm}^{-1}\end{array}$ & Pulse duration $/ \mathrm{ms}$ & $\begin{array}{c}\text { Exc. bandwidth } \\
\Delta v_{\mathrm{BW}} / \mathrm{Hz}\end{array}$ & Slice width $/ \mathrm{mm}$ \\
\hline${ }^{1} \mathrm{H}$ & $G 4$ & 25.6 & 800 & $\sim 11000$ & $\sim 1$ \\
${ }^{2} \mathrm{H}$ & $G 4$ & 51.1 & 4800 & $\sim 1700$ & $\sim 0.5$ \\
${ }^{7} \mathrm{Li}$ & $G 4$ & 51.1 & 1000 & $\sim 8500$ & $\sim 1$ \\
\hline
\end{tabular}

For standard, rectangular high power pulses the flip angle is set through the pulse duration. This duration cannot be varied for selective pulses without changing the excitation bandwidth. Hence, maximum intensity flip angles $\left(90^{\circ}\right)$ for soft pulses must be adjusted by setting the pulse power (amplitude of the radio frequency pulse). TopSpin enables the determination of the appropriate power for a desired flip angle at a certain pulse length by integrating the adiabatic shape of the soft pulse. The full set of parameters to obtain $90^{\circ}$ flip angles and $1 \mathrm{~mm}$ slice width is given in Table 3-3.

Table 3-3: Pulse shape, pulse duration and pulse power for relevant nuclei that achieve the desired $90^{\circ}$ flip angle.

\begin{tabular}{ccccc}
\hline Nucleus & Pulse shape & Pulse power $/ \mathrm{W}$ & Pulse duration $/ \mathrm{ms}$ & Flip angle $/{ }^{\circ}$ \\
\hline${ }^{1} \mathrm{H}$ & $G 4$ & 0.358 & 800 & 90 \\
${ }^{2} \mathrm{H}$ & $G 4$ & 3.93 & 4800 & 90 \\
${ }^{7} \mathrm{Li}$ & $G 4$ & 3.18 & 1000 & 90 \\
\hline
\end{tabular}

\section{Determination of the spatial detection sensitivity}

The active region of the NMR probe (given by the coil dimensions) is limit to a length of around $2 \mathrm{~cm}$. This allows excitation of $\sim 20$ slices à $1 \mathrm{~mm}$. Signal integrals $\left(I_{\mathrm{sig}}\right)$ of $90^{\circ}$ soft pulses are reduced to $\sim 5 \%$ compared to $90^{\circ}$ hard pulses (Figure 3-4 a). However, the sensitivity profile of the detection coil is not perfectly uniform, which has to be addressed through correction factors $f_{\mathrm{cs}}\left(I_{\mathrm{corS}}=f_{\mathrm{cs}} \cdot I_{\mathrm{sig}}\right)$. Therefore, homogeneous reference samples were measured and signal integrals were determined (Figure 3-4 b).
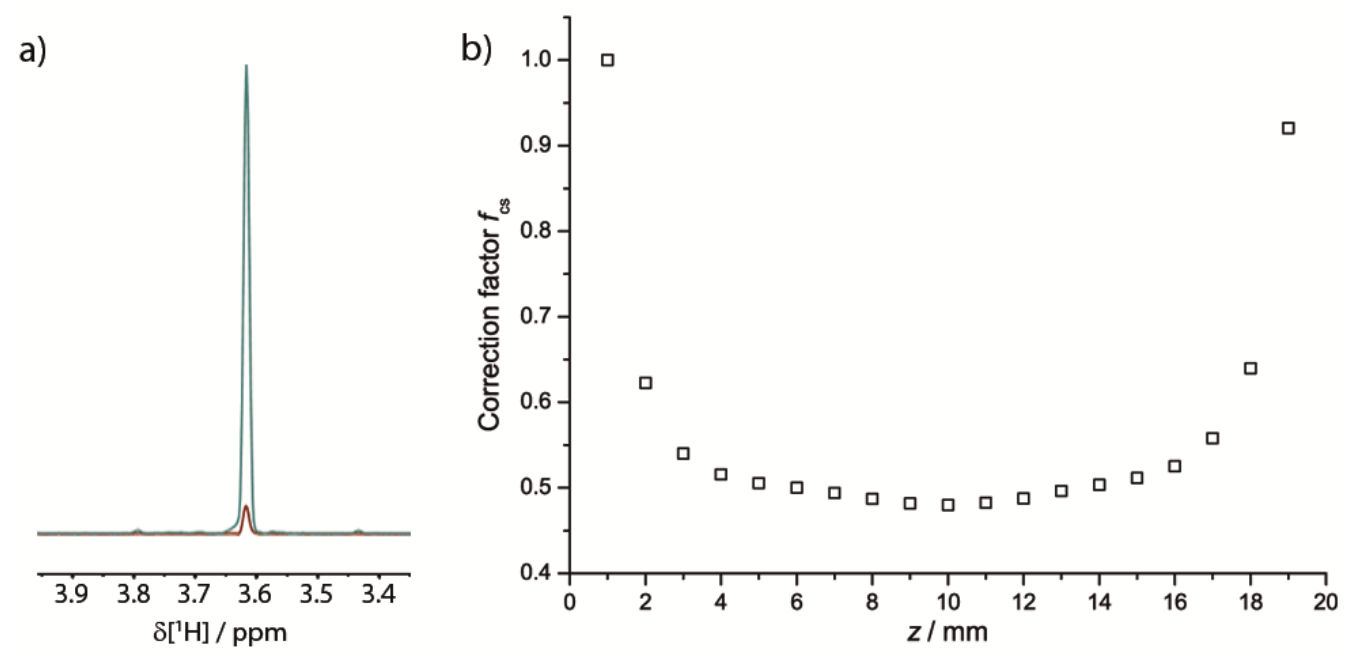

Figure 3-4: a) Comparison of a $90^{\circ}$ hard pulse (green) and a $1 \mathrm{~mm} \mathrm{G4} 90^{\circ}$ soft pulse (red). b) Correction factors $f_{\mathrm{cs}}$ for the integrals of 19 slices (top to bottom) for $1 \mathrm{~mm} \mathrm{G4} 90^{\circ}$ soft pulses. 
Note that in Figure 3-4 a) the intensity decreases but the full width at half maximum of the signal decreases as well. This increase of spectral resolution results from less magnetic imperfections along thinner slices in homogeneous samples. According to Figure 3-4 b) the detection coil exhibits mitigations especially at the outer regions. These mainly originate from the confined spatial dimensions of the coil and cannot be circumvented.

\subsection{Observation of Reactions}

Chemical reactions can be observed by monitoring substrates in a series of individual NMR spectra over time. The observation of fast reactions and short-lived intermediates has become possible through custom build NMR hardware and techniques such as stopped flow and RI NMR. ${ }^{[29]}$ In this thesis, a very different approach was made by utilizing the spatial resolution that can be achieved through SSE:

As described above, SSE has already been utilized to study diffusion of solvents and solutes into polymers. In particular, Pöppler et al. focused on inert crosslinked PS that can be swollen with toluene solutions containing highly reactive lithium amides. ${ }^{[37]}$ The fully equilibrated polymers (swollen over a sufficiently long period of time, usually several days) containing a reactive species is the basis for the innovative SSE reaction monitoring approach. Here, a further substrate is added on top of the gel and diffuses into the polymer to yield a reaction front between both compounds. This progress (Scheme 3-1) can be readily observed through SSE.

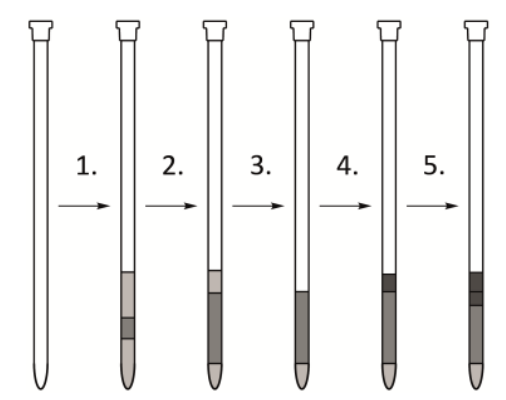

Scheme 3-1: Basic steps of sample preparation: 1. A crosslinked polymer (dark grey) is immersed in a solution which contains the reactive (lithium organic) species (bright grey). 2. The polymer is swollen (equilibrated) over a sufficiently long time period. This can be evaluated through homogeneous signal intensities via SSE as shown by Pöppler et al. 3. The supernatant solution above the polymer is removed. 4. A solution containing a further substrate (black) is added on top of the polymer. 5. The substrate diffuses into the gel. 6 . This is then followed by SSE over an appropriate period of time.

\section{Reaction between $n$ BuLi and PMDTA}

The lithiation of PMDTA with $n \mathrm{BuLi}$ was examined as a case study. PMDTA is usually applied as a donor base that allows disaggregation of lithium organic compounds into smaller aggregates. ${ }^{[15 a, 19,102]}$ The disaggregation and subsequent lithiation of substrates is usually performed at lowered temperatures to control the reactivity and delicate selectivity of the lithium organic compounds. Disaggregation at room temperature frequently leads to the undesired lithiation of the donor base. A most prominent 
example is $n \mathrm{BuLi}$ in THF which easily undergoes lithiation and ether cleavage. ${ }^{[103]} \mathrm{A}$ similar behaviour is documented for $n$ BuLi and PMDTA (Scheme 3-2): ${ }^{[104]}$

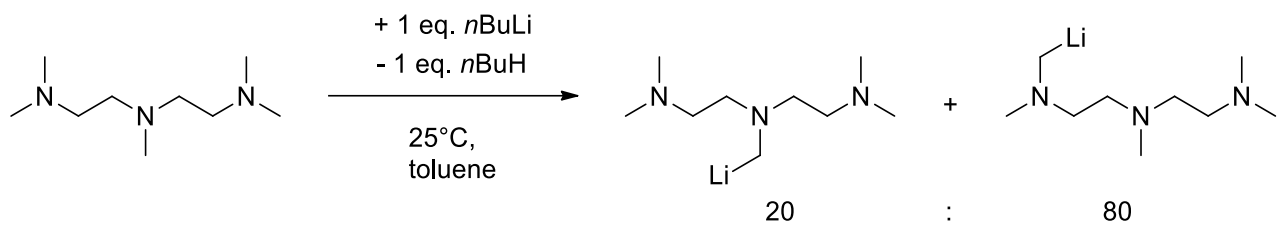

Scheme 3-2: The reaction between PMDTA and $n \mathrm{BuLi}$ in toluene at $25^{\circ} \mathrm{C}$ yields a mixture of lithiated PMDTA (20\% central methyl group, $80 \%$ terminal methyl groups lithiated).

Previous studies have shown that at room temperature and upon addition of one equivalent $n \mathrm{BuLi}$ a mixture of products results in which either the central methyl group (20\%) or one of the terminal methyl groups $(80 \%)$ of PMDTA is lithiated. ${ }^{[105]}$ This reaction was chosen as a case study to test the reaction observation approach:

According to Scheme 3-1, samples were prepared by immersing a cylindrical PS stick in a solution of $n \mathrm{BuLi}$ in toluene- $d_{8}$. Then PMDTA was added on top of the swollen polymer. Slice-selective ${ }^{1} \mathrm{H}$ and ${ }^{7} \mathrm{Li}$ NMR measurements started $\sim 3 \mathrm{~h}$ after preparation of the sample and went on for three days (experimental details on sample preparation and SSE parameters are described in the experimental section 5.1). Figure 3-5 shows a series of 19 slice-selective ${ }^{7} \mathrm{Li}$ NMR spectra as obtained three days after the addition of PMDTA to the polymer imbibed with $n$ BuLi.

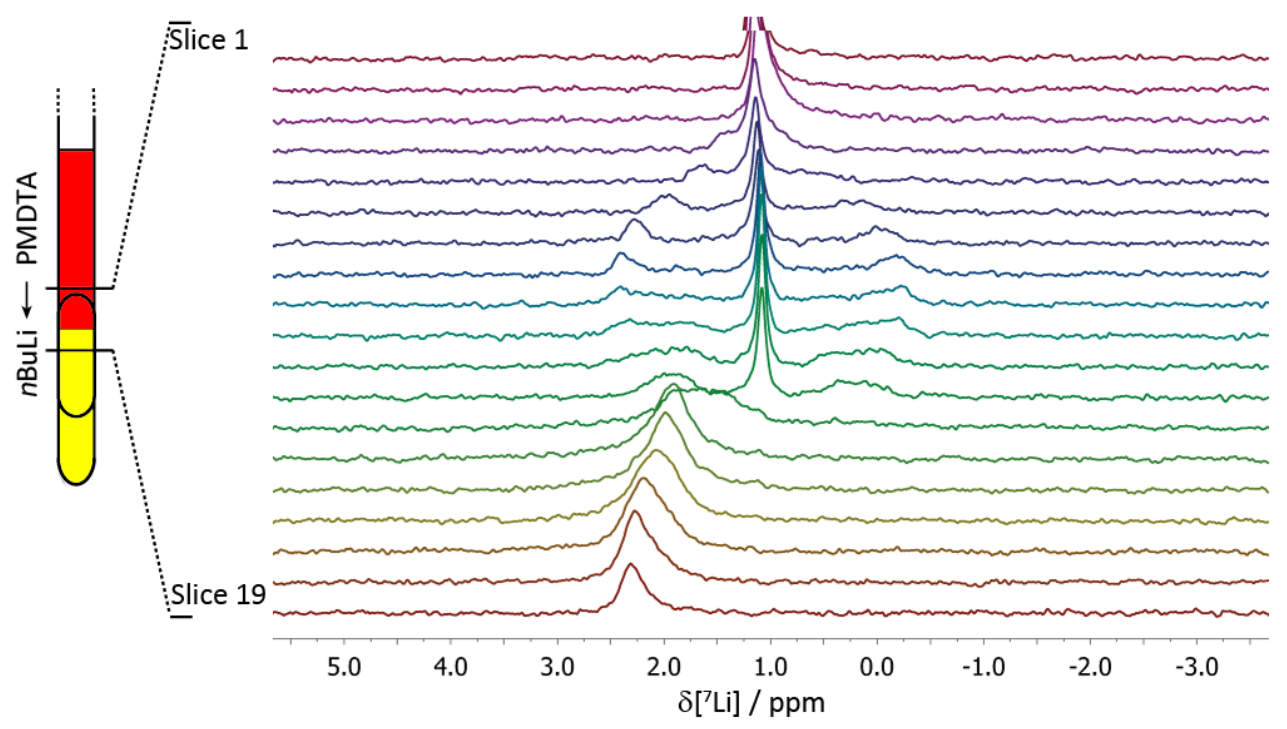

Figure 3-5: Slice-selective ${ }^{7} \mathrm{Li}$ NMR spectra (slices 1 to 19) of the reaction between $n \mathrm{BuLi}$ and PMDTA within a PS gel three days after the addition of PMDTA.

Slices 19 and 18 at the bottom of the active volume still show the signal of unreacted $n \mathrm{BuLi}$ at $2.3 \mathrm{ppm} .{ }^{[16,106]}$ In the upper slices 1-12 a narrow resonance at $1.1 \mathrm{ppm}$ is observed that can be assigned to the products of the lithiation (Scheme 3-1). It is impossible to discriminate the terminally and internally lithiated products since their chemical environments are almost identical and the narrow chemical shift range of ${ }^{7} \mathrm{Li}$ nuclei prohibit these minute distinctions. 
Slices 1-4 are located outside the polymer and, hence, only a singlet is observed. Satellite peaks in slices 5-12 arise from RQCs due to the partial alignment of the product molecules inside the stretched gel (vide infra). For $n \mathrm{BuLi}$ in slices 18 and 19 a singlet is observed since the $O_{\mathrm{h}}$ symmetry of the hexameric $\left[n \mathrm{Buli}_{6}\right.$ aggregate in toluene does not allow any alignment. ${ }^{[38]}$

Note that the size of the RQCs in slices 5-12 changes significantly. Various factors may contribute to this phenomenon: Most likely, the amount of strain within the gel is changing. ${ }^{[37,53]}$ In particular, the strain may decrease towards the surface between neat solvent and polymer. Accordingly, the alignment of molecules in these slices becomes weaker as can be observed for spectra of slices between 5 and 8 . This is strongly supported by ${ }^{2} \mathrm{H}$ SSE spectra and the observed splitting for their methyl deuterons (Figure 3-6).

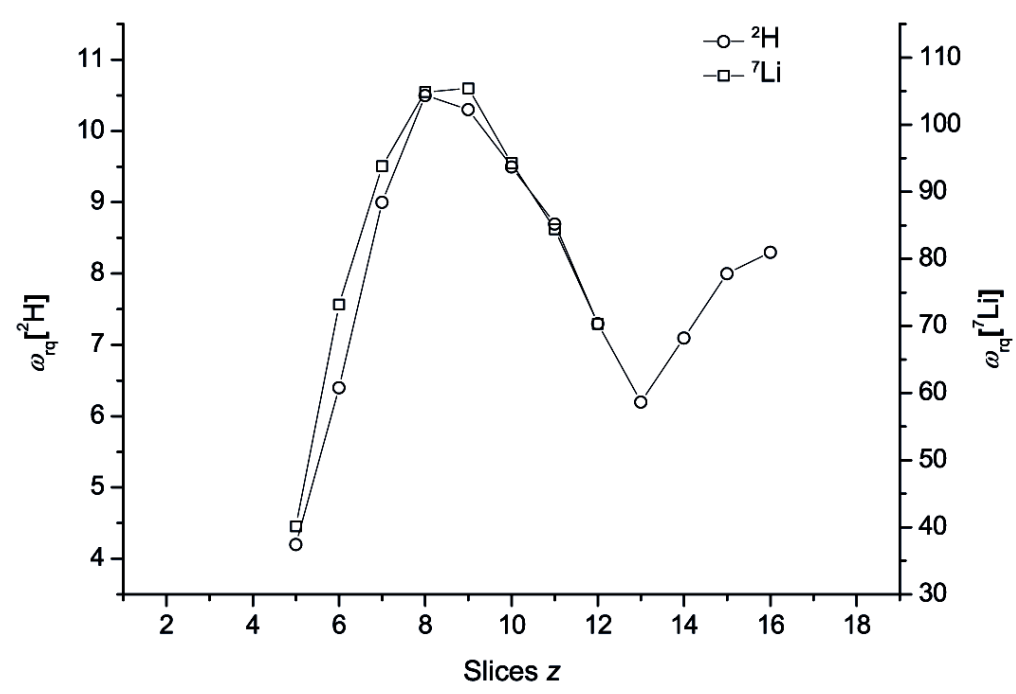

Figure 3-6: Correlation between slices and RQCs for ${ }^{2} \mathrm{H}$ of the methyl group in toluene- $d_{8}$ and ${ }^{7} \mathrm{Li}$ of the lithiation product.

Toluene does not take part in the reaction and can therefore serve as a probe for the alignment strength of the polymer. Figure 3-6 shows that the lithium specimen and toluene- $d_{8}$ experience proportional strain. Hence, it is reasonable that the change in ${ }^{7} \mathrm{Li}$ RQCs is due to changes in the alignment matrix. These changes presumably results from the exothermic reaction between $n \mathrm{BuLi}$ and PMDTA which disturbs the relaxed/stretched polymer network and results in the dip at the reaction front (slice 13).

Slices 9-17 in Figure 3-5 exhibit further interesting features: While the central transition is sharp in slices 9-12, the satellite transitions become increasingly broadened. This indicates a superposition of different RQCs which again mainly originate from varying alignment as supported by the ${ }^{2} \mathrm{H}$ SSE spectra of toluene- $d_{8}$ (though different, superimposed reaction products with similar ${ }^{7} \mathrm{Li}$ shift may exist). This non-equilibrium strain in the middle parts of the gel is most likely due to a response of the PS matrix upon the uptake of PMDTA. ${ }^{a}$

\footnotetext{
${ }^{a}$ Note that a plethora of possible parameters within hydrogels influences their swelling behaviour. These range from solute characteristics like different osmotic pressures to external stimuli such as the temperature. These responses are particularly relevant to the application of hydrogels in medicinal chemistry. ${ }^{[107]}$
} 
Additionally, RQCs are (in contrast to ${ }^{7} \mathrm{Li}$ chemical shifts) very sensitive towards changes of the EFG which is analogous to changing aggregation motifs. Hence, slices 9-12 may also be characterized by dynamical processes involving different aggregates as well as both lithiated products.

Slices 13-17 are characterized through the vanishing of satellites and increasingly broad central transitions which exhibits a changing chemical shift between 1.6 and $2.3 \mathrm{ppm}$. This impressively marks the reaction front between PMDTA and $n \mathrm{BuLi}$. It must be highlighted, that the reaction between $n \mathrm{BuLi}$ and PMDTA is strongly exothermic. ${ }^{[104]}$ Hence, samples without a polymer gel exhibit convection and uncontrolled mixing of both compounds. Within the PS matrix $n \mathrm{BuLi}$ is practically immobilized and a remarkably distinct reaction front results from the predominant diffusion of the PMDTA molecules. However, the lack of resolved RQCs for ${ }^{7} \mathrm{Li}$ in these slices may stem from a decreased degree of alignment through increased temperature. This effect cannot be fully separated from dynamic processes that constitute the lithiation and which are in the focus of interest. Therefore, further conclusions must be drawn from the series of 19 slice-selective ${ }^{1} \mathrm{H}$ NMR spectra of the same sample (Figure 3-7 a).
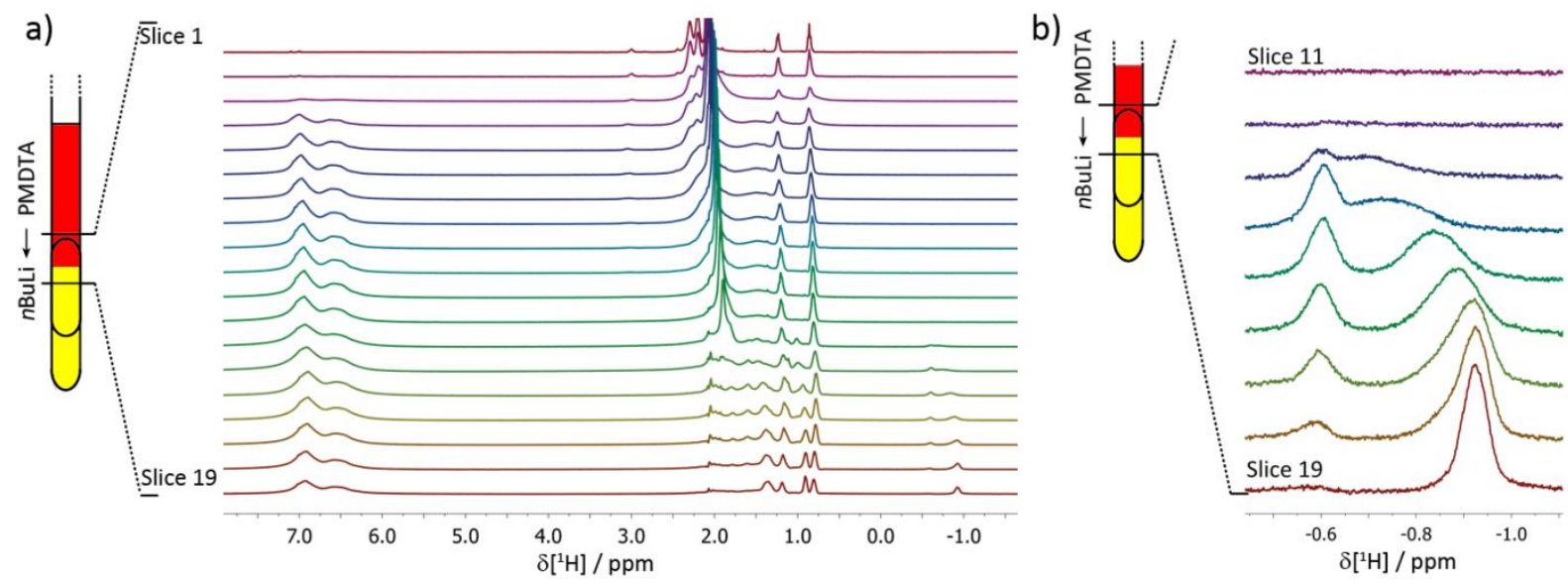

Figure 3-7: a) Slice-selective ${ }^{1} \mathrm{H}$ NMR spectra (slices 1-19) of the reaction between $n$ BuLi and PMDTA within the PS gel, three days after addition of PMDTA. b) Enlarged excerpt of the same spectrum for slices 11-19 at the high field limit of the spectrum.

In contrast to ${ }^{7} \mathrm{Li},{ }^{1} \mathrm{H}$ gives relatively crowded spectra with broad polymer resonances between 6.1 and $7.2 \mathrm{ppm}$ and around $2 \mathrm{ppm}$. Especially between 0.6 and $2.3 \mathrm{ppm}$ signals from various reaction components and the polymer disable attribution of single resonances. However, the isolated region between -0.5 and $-1.1 \mathrm{ppm}$ is quite informative (Figure 3-7 b). This part includes the exposed $n \mathrm{BuLi} \alpha-$ $\mathrm{CH}_{2}$ protons $(-0.92 \mathrm{ppm})^{[16,106]}$ and a number of interesting features: The most remarkable characteristic is the appearance of an additional, non-shifting resonance at $-0.59 \mathrm{ppm}$ in slices $18-13$ with a maximum intensity in slice 15 . The connection between the reaction progress and the appearance of this peak can be easily illustrated through a comparison of its integral and the integral of the remaining $n \mathrm{BuLi} \alpha-\mathrm{CH}_{2}$ signal (although this signal is shifting, it can be fully attributed to $n \mathrm{BuLi}$, vide infra) over the course of three days (Figure 3-8). 


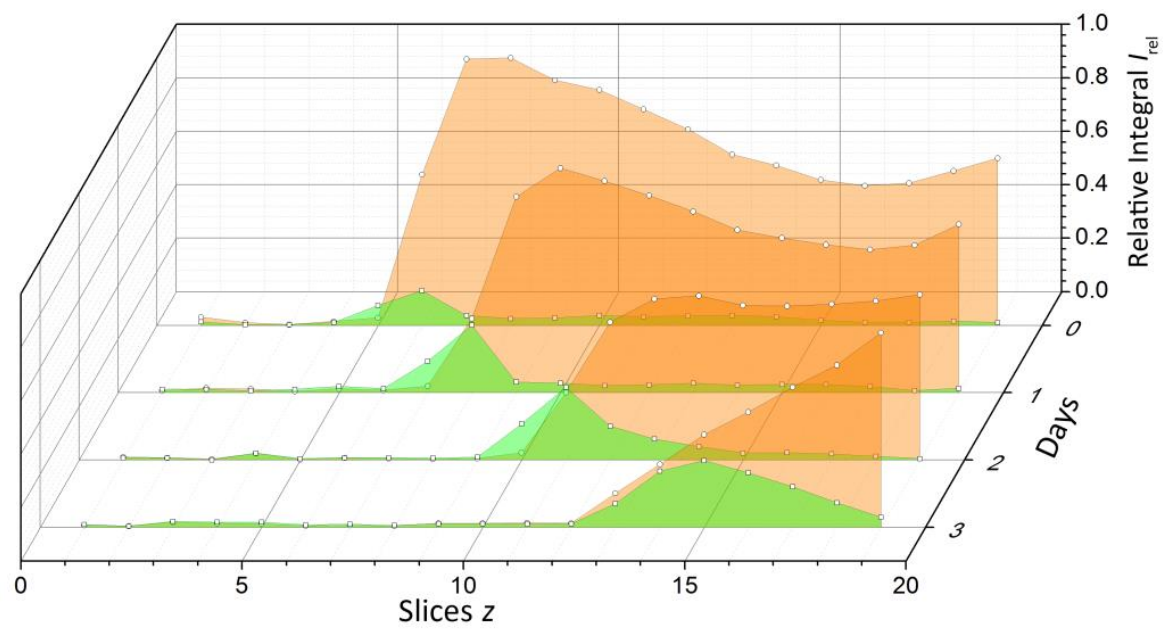

Figure 3-8: Relative integrals for the ${ }^{1} \mathrm{H}$ NMR signals of the intermediary species at $-0.59 \mathrm{ppm}$ (green) and the signal of the $n \mathrm{BuLi} \alpha-\mathrm{CH}_{2}$ protons (orange) as function of slice number, $3 \mathrm{~h}, 1,2$ and 3 days after addition of PMDTA to $n$ BuLi inside the pre-swollen PS gel. All signals high-field to $-0.59 \mathrm{ppm}$ were attributed to $n \mathrm{BuLi}$.

Figure 3-8 clarifies the motion of the reaction front, where the $n \mathrm{BuLi} \alpha-\mathrm{CH}_{2}$ signal (orange) vanishes and the signal at -0.59 ppm appears. The latter builds up and decays exclusively at the reaction front which impressively indicates its intermediate character. In fact, this signal as well as further signals in the crowded part of the spectra (Figure 3-9 a) can be assigned to the complex $\left[(n \mathrm{BuLi})_{2} \mathrm{PMDTA}\right]_{2}$ which was first described by Gessner and Strohmann in 2007. ${ }^{[104]}$
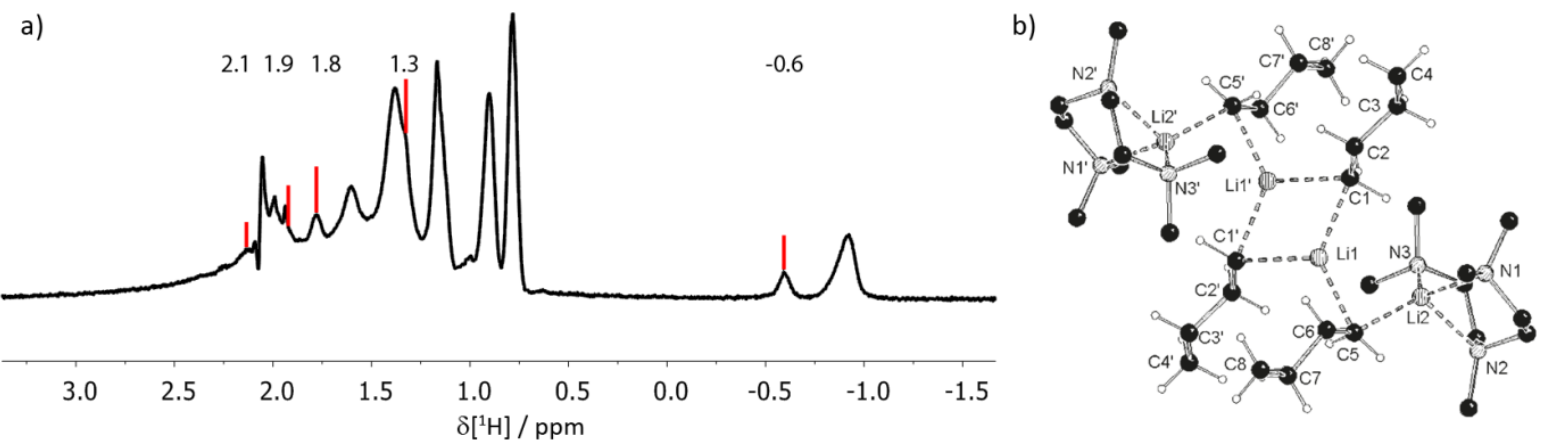

Figure 3-9: a) NMR spectrum of slice 17 after $3 \mathrm{~d}$ with explicit values that can be attributed to $\left[(n \mathrm{BuLi})_{2} \mathrm{PMDTA}\right]_{2}$ according to the report from Gessner and Strohmann. ${ }^{[104]}$ b) Crystal structure of $\left[(n \mathrm{BuLi})_{2} \mathrm{PMDTA}\right]_{2}$. Crystal structure reprinted with permission from reference [104]. Copyright 2007 Wiley VCH.

In their study, the authors found that remarkably stable crystals can be obtained from quenched reactions between $n \mathrm{BuLi}$ and PMDTA. The structure of the crystallized molecules was determined via XRD (Figure 3-9 b) and single crystals were subsequently solved in toluene- $d_{8}$ to record ${ }^{1} \mathrm{H}$ NMR spectra. Note that the crystallization as well as XRD and NMR measurements of the stable intermediate were performed at $223 \mathrm{~K}$ while the authors observed that the lithiation inevitably starts above $253 \mathrm{~K}$. Additional comprehensive quantum chemical calculations on $\left[(n \mathrm{BuLi})_{2} \mathrm{PMDTA}\right]_{2}$ and a hypothetical monomeric [ $(n \mathrm{BuLi}) \mathrm{PMDTA}]$ species together with corresponding transition states between both aggregates and the lithiated product unambiguously indicated that $\left[(n \mathrm{BuLi})_{2} \mathrm{PMDTA}\right]_{2}$ is the crucial intermediate in the lithiation of PMDTA. ${ }^{[04]}$ 
Figure 3-8 displays several further features: While $3 \mathrm{~h}$ after the addition of PMDTA to the pre-swollen gel, the reaction front is relatively sharp with little formation of $\left[(n \mathrm{BuLi})_{2} \mathrm{PMDTA}\right]_{2}$, it becomes significantly blurred as PMDTA moves downward with time. This concentration gradient is in accordance with what would be expected from diffusion into/within a gel. ${ }^{[108]}$ Furthermore, the dip in the $n \mathrm{BuLi}$ concentration which is initially observed in the center of the gel most likely arises from slow and incomplete diffusion of $n \mathrm{BuLi}$ into the polymer during sample preparation. ${ }^{\mathrm{a}}$ This again underlines that the $n \mathrm{BuLi}$ hexamer $\left(M_{\mathrm{W}}=383 \mathrm{~g} \cdot \mathrm{mol}^{-1}\right)$ may be regarded as rather static compared to the faster diffusing PMDTA molecules $\left(M_{\mathrm{W}}=173 \mathrm{~g} \cdot \mathrm{mol}^{-1}\right)$.

The concluding remarks on reaction observation via SSE must address another interesting feature of Figure 3-7 b) that has not been fully acknowledged yet: the $n \mathrm{BuLi}$ resonance at $-0.92 \mathrm{ppm}$ is shifting downfield and significantly broadens from slice 19 to 13 . It is obvious that, due to the diffusion of PMDTA and the resulting concentration gradient, different ratios between the donor base and $n \mathrm{BuLi}$ must occur in the slices along the NMR tube. Hence, the change in shift resembles the course of a titration experiment in which two compounds ( $n \mathrm{BuLi}$ and PMDTA) form another $\left[(n \mathrm{BuLi})_{\mathrm{x}}(\mathrm{PMDTA})_{\mathrm{y}}\right]$ complex with unknown stoichiometry. Within this work, only a hypothesis can be made about the aggregate:

The shifting signal seemingly approaches the resonance of the dimeric intermediate at $-0.59 \mathrm{ppm}$ which loosely indicates similar chemical environments for the unknown $\left[(n \mathrm{BuLi})_{\mathrm{x}}(\mathrm{PMDTA})_{\mathrm{y}}\right]$ and $\left[(n \mathrm{BuLi})_{2} \mathrm{PMDTA}\right]_{2}$. A gradual breaking down of $[n \mathrm{BuLi}]_{6}$ towards the monomeric [( $\left.\left.n \mathrm{BuLi}\right) \mathrm{PMDTA}\right]$ adduct has been proposed several times, but not yet been experimentally confirmed. ${ }^{[15 a]}$ The experimental findings shown above indicate this disaggregation process.
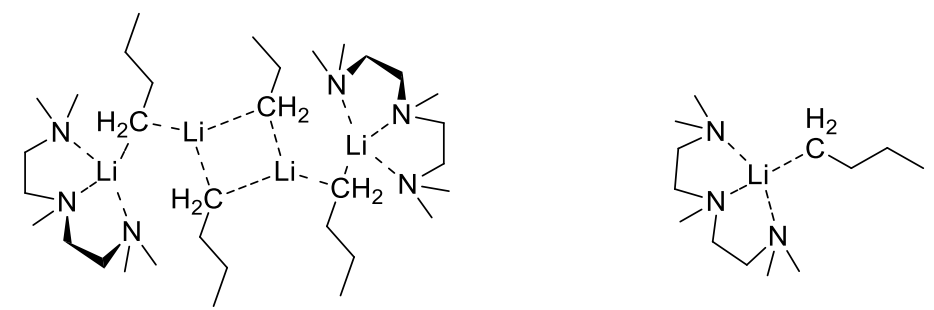

Scheme 3-3: Proven intermediate $\left[(n \mathrm{BuLi})_{2} \mathrm{PMDTA}_{2}\right.$ (left) and potential monomeric $[(n \mathrm{BuLi}) \mathrm{PMDTA}]$ adduct (right).

As already stated before, the latter observations are made through a setup that resembles an elegant titration experiment. The diffusion of PMDTA leads to different ratios of PMDTA and $n$ BuLi along the NMR tube. This was correlated with changing chemical shifts of nuclei in the corresponding slices. This approach is optimized and exploited in the application of SSE as a single-shot titration experiment.

\footnotetext{
${ }^{a}$ As already mentioned, the swelling behaviour of the polymer is not in the focus of this thesis. However, preliminary studies showed that the species and the amount of solute heavily influence these dynamics. The swelling of polymers with neat solvent leads to extreme expansion of the gel and frequent polymer ripping. Different osmotic pressures as well as different diffusion characteristics are evident explanations for this interesting phenomenon.
} 


\subsection{Single-Shot Titration}

Chemical shift titration is a powerful method to determine the stoichiometry and stability constants of complexes in coordination, supramolecular and medicinal chemistry. ${ }^{[109]}$ In such an experiment, the chemical shift of a particular resonance attributed to one component (e.g. the metal, guest or target) is monitored in a series of NMR spectra, while the concentration of the other component (e.g. the host or ligand) is systematically varied. Rather than incrementing the concentration step-by-step as in the traditional approach, the experiment on $n \mathrm{BuLi}$ and PMDTA (section 3.2) showed the possibility to perform titrations in a single experiment.

The most obvious drawbacks of a complete adoption of the approach described above are the timeconsuming sample preparation (several days of swelling) and the challenging measurements within the PS gel: gel samples require extensive manual shimming on the free induction decay (FID) to achieve sufficiently homogeneous signals and resolution (also see experimental section 5.1). These pitfalls are accepted for SSE in reaction observation since the gel is essential to prevent the convection (only this allows the persistent formation of a reaction front) and allows the detection of anisotropic parameters (which add structural information). However, this procedure would be less applicable, simplistic and straightforward than standard titration experiments on several different samples with changing concentrations. The decisive step to achieve a smart single-shot titration experiment is to dismiss the gel and instead layer two plain compounds in solutions (Scheme 3-4). In the present study, this was achieved by solidifying the bottom compound through a decrease in temperature.

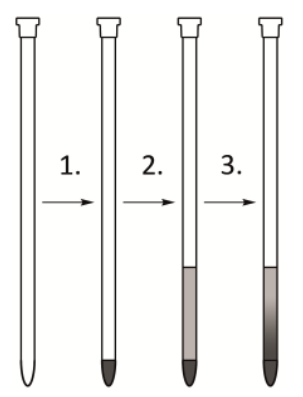

Scheme 3-4: Basic steps of sample preparation: 1. The first compound (neat) is filled into the NMR tube. 2. The second compound, immersed in deuterated solvent, is carefully added on top of the first compound. 3 . The compound at the bottom then diffuses into the solution above. 4 . This can be readily observed through SSE.

\section{Titration of Li+ with 12-crown-4}

As a case study the titration of $\mathrm{Li}^{+}$with 12 -crown- 4 was performed. ${ }^{[110]} \mathrm{LiClO}_{4}$ was used as a $\mathrm{Li}^{+}$source since it is highly soluble in organic solvents such as acetonitrile.
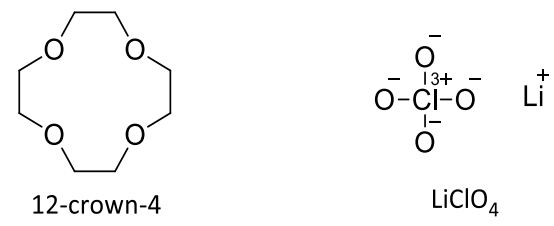

Scheme 3-5: Compounds for the titration experiment. 
Crown ethers are versatile chemical compounds that are especially known for their selective binding towards metal cations. ${ }^{[11]}$ This enabled their application as crucial detection moieties in molecular sensors ${ }^{[112]}$ and as transfer agent in phase transfer catalysis ${ }^{[113]}$. The ether 12-crown-4 has a particularly high affinity towards $\mathrm{Li}^{+}$and the complexation is well characterized. ${ }^{[110]}$ Hence, this complexation was chosen as a case study to test the single-shot titration approach:

According to Scheme 3-4 samples were prepared by first filling liquid 12-crown-4 in an NMR tube. The sample was then cooled down to $5^{\circ} \mathrm{C}$ to solidify the ether (melting point $16^{\circ} \mathrm{C}$ ). Subsequently, a solution of $\mathrm{LiClO}_{4}$ in acetonitrile- $d_{3}$ was layered on top of the solid ether. This procedure prevents mixing of both components (without a gel) prior to measurements, and only inside the NMR magnet $\left(25^{\circ} \mathrm{C}\right)$ the ether melts and slowly diffuses into the $\mathrm{LiClO}_{4}$ solution. Diffusion of $\mathrm{LiClO}_{4}$ into the ether reservoir occurs likewise, but with little impact on the ${ }^{7} \mathrm{Li}$ concentration due to the $\sim 9: 1$ volume ratio and the lowered concentration gradient ( $5.1 \mathrm{~mol} / \mathrm{L} \mathrm{LiClO}_{4}$ solution as opposed to bulk ether). Approximately 3 , 6 , and $9 \mathrm{~h}$ after sample preparation, slice-selective ${ }^{1} \mathrm{H}$ and ${ }^{7} \mathrm{Li}$ NMR measurements were performed (experimental details on sample preparation and SSE parameters are described in the experimental section 5.1). Figure 3-10 shows the series of 19 slice-selective ${ }^{1} \mathrm{H}$ NMR spectra recorded after $6 \mathrm{~h}$ and the corresponding normalized integrals of the ${ }^{1} \mathrm{H}$ signal in each slice.

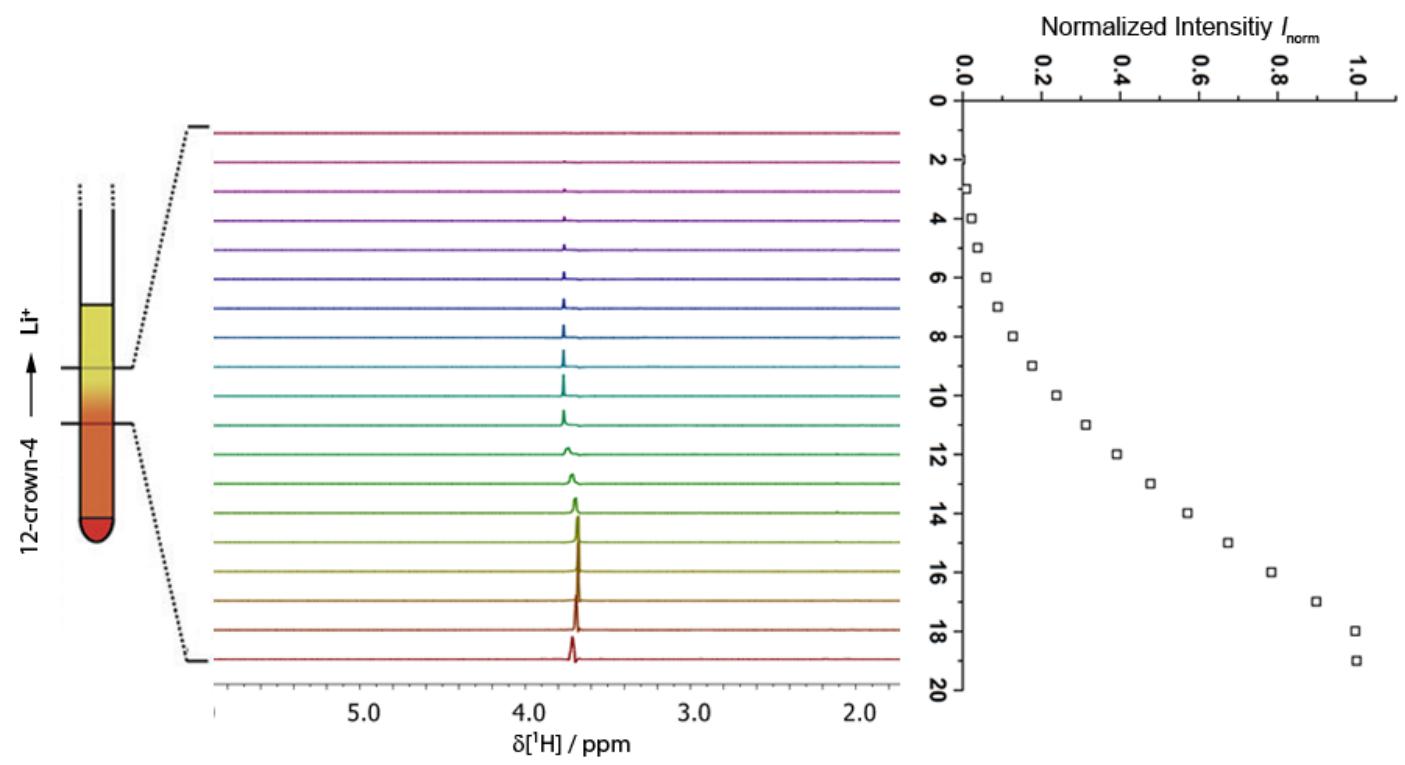

Figure 3-10: Slice-selective ${ }^{1} \mathrm{H}$ NMR spectra of 12-crown-4, $6 \mathrm{~h}$ after sample preparation and the corresponding concentration profile from corrected and normalized Intensities.

The single chemical shift of the ether signal exhibits only minor changes and is therefore not suitable for any further analysis. However, signal intensities decrease from bottom to top indicating the diffusion of 12-crown-4 in the same direction. Integration of the signals for all 19 slices and subsequent correction according to the sensitivity of the detection coil (see section 3.1) illustrates a smooth concentration gradient. In contrast to slice-selective ${ }^{1} \mathrm{H}$ measurements, ${ }^{7} \mathrm{Li}$ spectra exhibit a more pronounced change of the chemical shift for the $\mathrm{Li}^{+}$ion (Figure 3-11). 


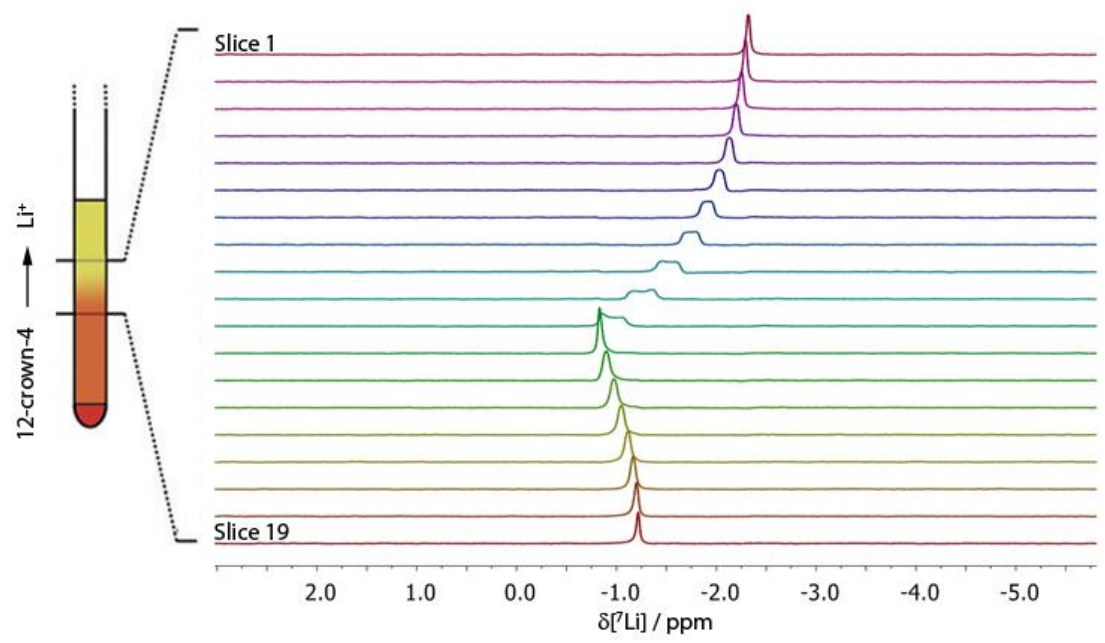

Figure 3-11: Slice-selective ${ }^{7} \mathrm{Li}$ NMR spectra of $\mathrm{LiClO}_{4}$ in acetonitrile- $d_{3}$ in the presence of a gradient of 12-crown$4,6 \mathrm{~h}$ after sample preparation.

The signals for ${ }^{7} \mathrm{Li}$ were integrated and corrected. These values are approximately constant and thereby confirm that almost no diffusion of $\mathrm{LiClO}_{4}$ occurs. Slice 1 at the top of the active volume shows a narrow ${ }^{7} \mathrm{Li}$ resonance at $-2.3 \mathrm{ppm}$ which is typical for $\mathrm{Li}^{+}$coordinated by four acetonitrile molecules. ${ }^{[110]}$ As the ether concentration increases from slice 1 to 19 (Figure 3-10), the ${ }^{7} \mathrm{Li}$ resonance is shifted downfield and reaches a maximum $(-0.8 \mathrm{ppm})$ in slice $12 \cdot{ }^{[110]}$ Note that the ${ }^{7} \mathrm{Li}$ resonances in slices 6 to 11 are increasingly broadened. This is due to the strongly changing ether concentration within these slices which can only be monitored as superimposed (rectangular weighted sums) signals. This broadening could be overcome by further decreasing the slice width (see section 3.1) at the cost of sensitivity. After a maximum downfield shift is observed in slice 12, the signal moves upfield again until it reaches $-1.2 \mathrm{ppm}$ in slice $19 .{ }^{[110]}$

Quantitative analysis ${ }^{[114]}$ of titration data depends on well-determined concentrations of all components involved. Calibration curves that correlate absolute signal intensity $I_{\mathrm{abs}}$ and concentration are a common measure to obtain these values. Instrument specific calibration curves for ${ }^{1} \mathrm{H}$ and ${ }^{7} \mathrm{Li}$ are documented in the experimental section 5.3 and were applied to convert the measured and corrected absolute integrals for each slice-selective ${ }^{1} \mathrm{H}$ NMR and ${ }^{7} \mathrm{Li}$ NMR spectrum into concentrations. Finally the specific ether/lithium ratio of a slice could be assigned to each corresponding ${ }^{7} \mathrm{Li}$ chemical shift (Figure 3-12). 


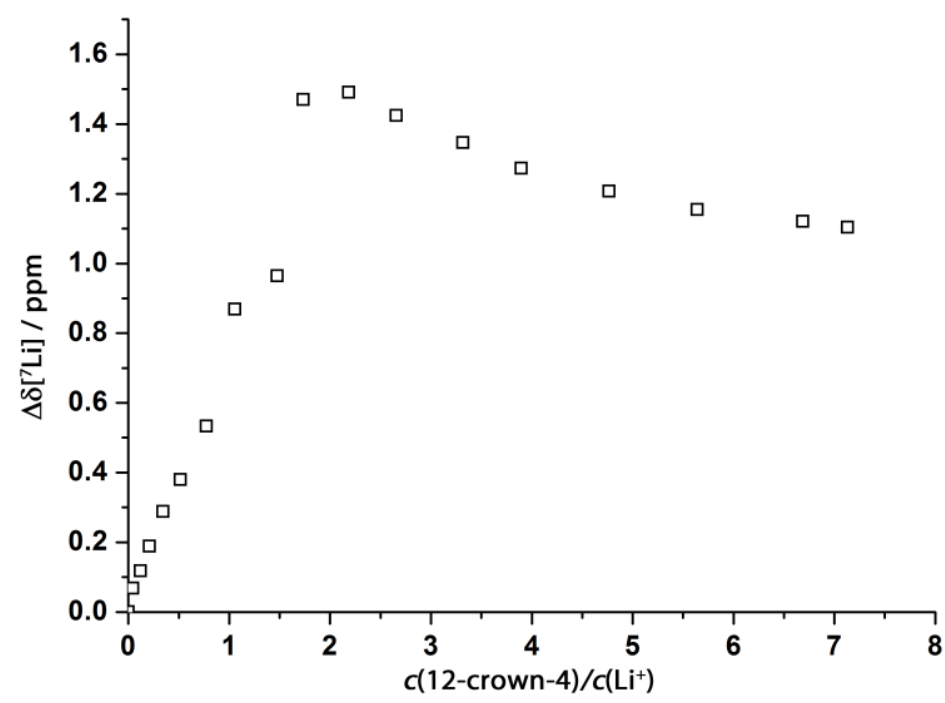

Figure 3-12: Chemical shift difference $\Delta \delta\left[{ }^{7} \mathrm{Li}\right]$ between pure $\mathrm{Li}^{+}$in acetonitrile and varying amounts of 12 -crown4 as measured $6 \mathrm{~h}$ after preparation of the sample.

Figure 3-12 further illustrates the changing ${ }^{7} \mathrm{Li}$ resonance in the presence of different ether/lithium ratios which qualitatively matches the titration curve of $\mathrm{Li}^{+}$by 12 -crown- 4 as provided by Masiker (Figure 3-13). ${ }^{[10 b]}$

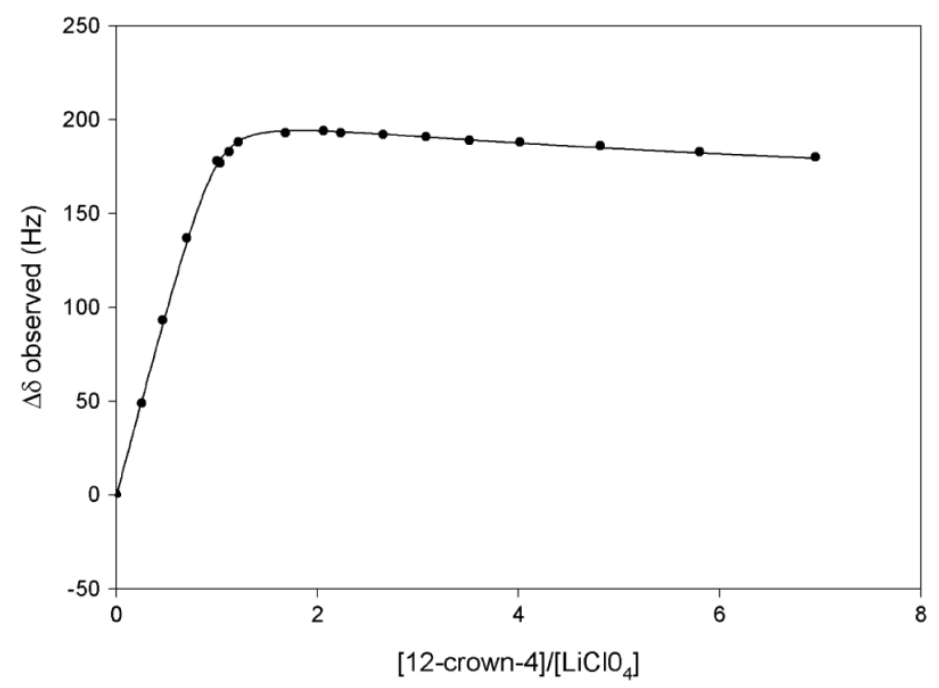

Figure 3-13: Chemical shift difference $\Delta \delta\left[{ }^{7} \mathrm{Li}\right]$ between pure $\mathrm{Li}^{+}$in acetonitrile and varying amounts of 12-crown4. Reprint with permission from reference [110b]. Copyright 2010 John Wiley and Sons.

The authors verified a 1:2 binding model between $\mathrm{Li}^{+}$and 12-crown-4 in which lithium $(\mathrm{M})$ is stepwise coordinated by the ether $(\mathrm{L})$ resulting in a sandwich structure $\left(\mathrm{ML}_{2}\right)$ at higher ether/lithium ratios. ${ }^{[110]}$ For the SSE attempt decreasing the initial concentrations is the first choice to obtain better resolved results since the concentration gradient would be more shallow which increases the number of sampled data points for low ratios and, additionally, reduces peak widths. Finally, reducing slice widths by decreasing the excitation bandwidth would allow the fine tuning of peak resolution and once again sampling of more data points.

Meanwhile, the idea of utilizing concentration gradients and SSE for titration experiments was picked up and further improved by Mitrev et al. in the group of Jeannerat. ${ }^{[115]}$ In their study, the authors based 
experiments on the use of agar gel samples in which one compound was dispersed. The second compound is the layered on top of the gel and subsequently effects the concentration gradient through diffusion into the gel. This approach is very similar to the $n \mathrm{BuLi}$ PMDTA reaction observation in PS as described above. However, in the case of this titration experiments, agar has several advantages in comparison to a crosslinked polymeric gel such as PS: the authors state that the agar "gels have a remarkable property of causing no observable changes in the chemical shifts and lineshapes compared to normal aqueous solutions". The gel matrix is not visible in the NMR spectrum due to its "small quantity and favourable relaxation properties" and gel samples can be very fast and easily prepared by mixing the stationary compound, water and granular agar and applying "a simple heating/cooling cycle". ${ }^{[15]}$ Agar is commonly used in gel-electrophoresis ${ }^{[116]}$ for the separation of compounds in mixtures. This led to a case study in which SSE is exploited as a tool to perform qualitative chromatography.

\subsection{Qualitative Chromatography}

Characterization of complex mixtures is increasingly important for analytical chemists. ${ }^{[46]}$ Liquid chromatography and NMR can be directly coupled, e.g. via flow probes, enabling on-the-fly separation and detection of mixture compounds. ${ }^{[17]}$ Additionally, DOSY allows qualitative separation of mixture compounds according to self/tracer diffusion coefficients. ${ }^{[18]}$ This approach can be further enhanced by the application of diffusion affecting surfactants, complexing agents and likewise which is known as matrix-assisted DOSY. ${ }^{[119]}$ However, it must be emphasized that tracer diffusion (most commonly described through the Stokes-Einstein equation ${ }^{[32]}$ ) is different from chemical diffusion along a concentration gradient as described through Fick's laws ${ }^{[120]}$ (both become only identical at infinite dilution $\left.^{[121]}\right)$. The applications shown above illustrate that chemical diffusion can be readily observed through SSE. Hence, SSE can be utilized as part of a complementary and expedient method to achieve a chromatographic separation. The key to achieve this separation is the preparation of a stationary phase which grants homogeneous starting conditions (all compounds start diffusing at the same time at the same boundary) and sufficient separation of compounds as they diffuse into the gel (Scheme 3-6).

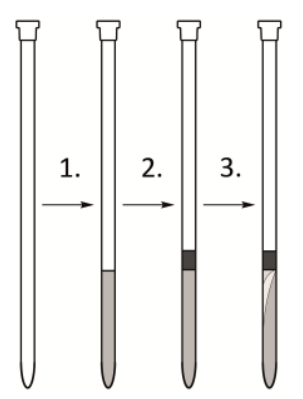

Scheme 3-6: Basic steps of sample preparation: 1. The stationary phase is prepared within the NMR tube. 2. A mixture of compounds to separate is added on top of the stationary phase. 3 . The compounds start diffusing into the stationary phase at different rates. 4 . The diffusion progress can be readily observed through SSE. 


\section{Separation of amino acids L-aspartic acid, L-histidine, L-tyrosine and L-valine in agarose}

As a case study the separation of amino acids L-aspartic acid (Asp, acidic), L-histidine (His, basic), Ltyrosine (Tyr, polar/neutral) and L-valine (Val, apolar) within agar hydrogel was examined (Figure 3$14)$.

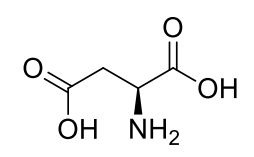

Asp, $133.1 \mathrm{~g} / \mathrm{mol}$<smiles>N[C@@H](Cc1ccc(O)cc1)C(=O)O</smiles>

Tyr, $181.2 \mathrm{~g} / \mathrm{mol}$<smiles>N[C@@H](Cc1c[nH]cn1)C(=O)O</smiles>

$\mathrm{His}, 155.2 \mathrm{~g} / \mathrm{mol}$<smiles>CC(C)C(N)C(=O)O</smiles>

Val, $117.2 \mathrm{~g} / \mathrm{mol}$

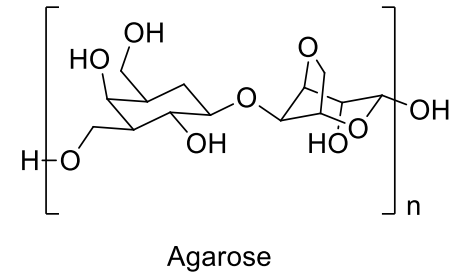

Agarose

Figure 3-14: Amino acids that were used in the qualitative chromatography through SSE case-study, from left top to right bottom: L-aspartic acid, L-His, L-Tyr and L-Val. Additionally the structure of agarose as main component of agar is shown on the right.

The amino acids are similar in molecular weight (117.2 to $181.2 \mathrm{~g} / \mathrm{mol})$ and soluble in slightly basic water. As already stated above agar gel has been described as a beneficial diffusion controlling medium in an elegant titration experiment by Mitrev et al. ${ }^{[115]}$ Agar consists of linear agarose and agaropectin molecules dispersed between the agarose chains. When dissolved in water a so-called heterogeneous gel is formed in which strong inter-polymer interactions hinder the polymer chains from extensive movements. ${ }^{[108 b]}$ The polymer is quasi static and spin relaxation is very fast, which is the origin of the NMR silence as observed by Mitrev et al. In contrast, PS is homogeneous and its polymer chains move less restricted effecting the residual polymer signals as observed in section 3.2.

According to Scheme 3-6 samples were prepared by first immersing agar in $\mathrm{D}_{2} \mathrm{O}$ within an NMR tube. The suspension was then heated until a homogeneous gel resulted. An equimolar mixture of L-aspartic acid, L-His, L-Tyr and L-Val in slightly alkaline $\mathrm{D}_{2} \mathrm{O}$ was added on top of the gel sample and SSE spectra were recorded immediately afterwards as well as 2, 6 and $10 \mathrm{~h}$ later. This procedure was repeated for gel samples with varying amounts of agar (weight-percentages of 2,3 and $5 \mathrm{wt} \%$ ), details on sample preparation and SSE parameters are described in the experimental section 5.1).

All spectra were then processed and integrals of distinct signals (L-Asp 2.20 ppm, L-His 7.54 ppm, L-Tyr $6.48 \mathrm{ppm}, \mathrm{L}-\mathrm{Val} 0.79 \mathrm{ppm}$ ) in each slice were measured and corrected using homogeneous reference samples (Figure 3-15). 


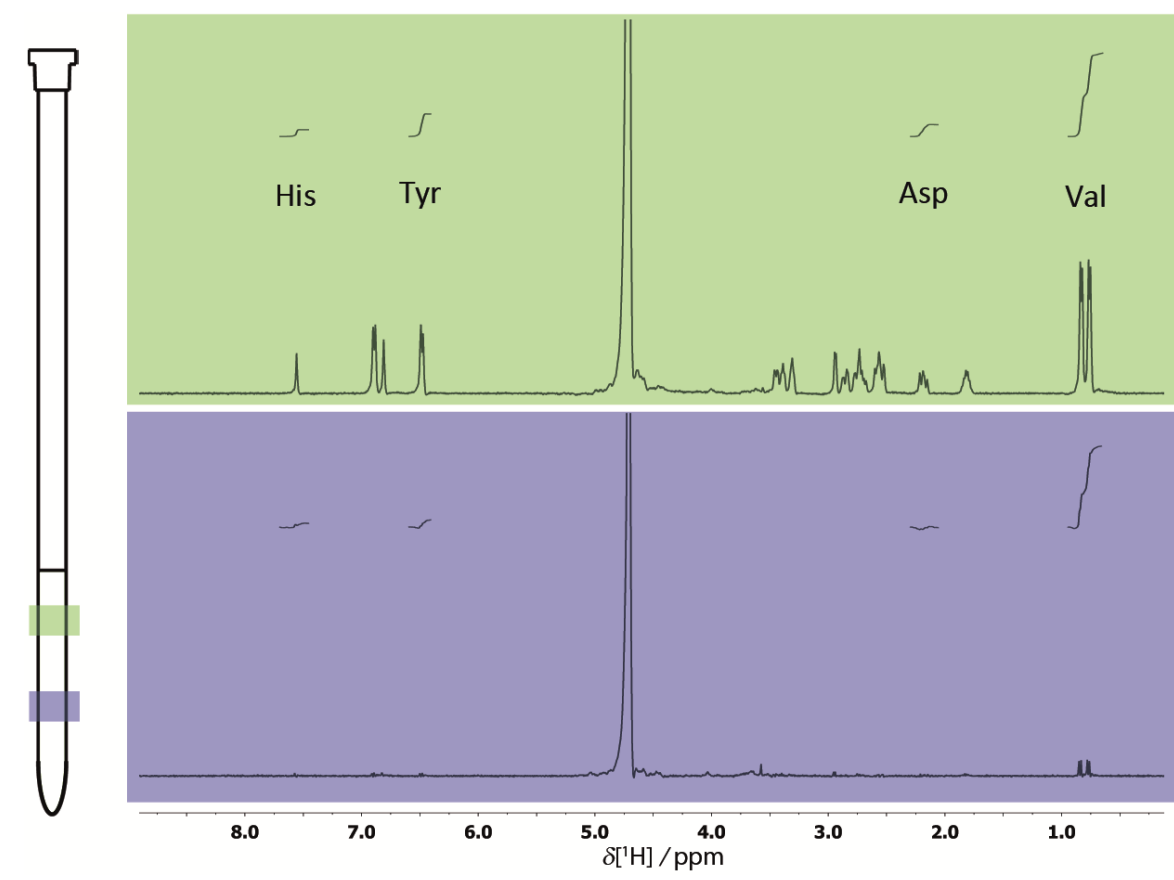

Figure 3-15: Spectra of slice 3 (top) and 10 (bottom) in the $3 \mathrm{wt} \%$ agar sample as obtained $6 \mathrm{~h}$ after preparation of the sample. From left to right integrals are marked for: His, Tyr, Asp and Val.

These spectra already show a pronounced decrease of signal intensity from top to bottom. Especially the signal for L-Val can be easily examined by eye, and is thereby recognized as the fastest diffusing species of the amino acid mixture. To achieve a reliable quantitative evaluation for the diffusion of the observed amino acids, concentration profiles were prepared from the measured intensities. Therefore, the integral $I_{0}$ of the slice $z_{0}=0 \mathrm{~mm}$ which corresponds to the boundary between agar and neat amino acid mixture in $\mathrm{D}_{2} \mathrm{O}$ was determined. Practically, $z_{0}$ was chosen as the slice that exhibits half of the maximum signal intensity of all SSE spectra obtained immediately after preparation. At this point, amino acid diffusion into the gel is still negligible.

Since integral $I$ and concentration $c$ are proportional, their ratios $I / I_{0}$ and $c / c_{0}$ involving the integral and concentration at the boundary $\left(I_{0}\right.$ and $\left.c_{0}\right)$, respectively, is equal. Thus, profiles of the normalized concentration $c / c_{0}$ against the slice $z$ can be directly prepared from the integrals (Figure 3-16).

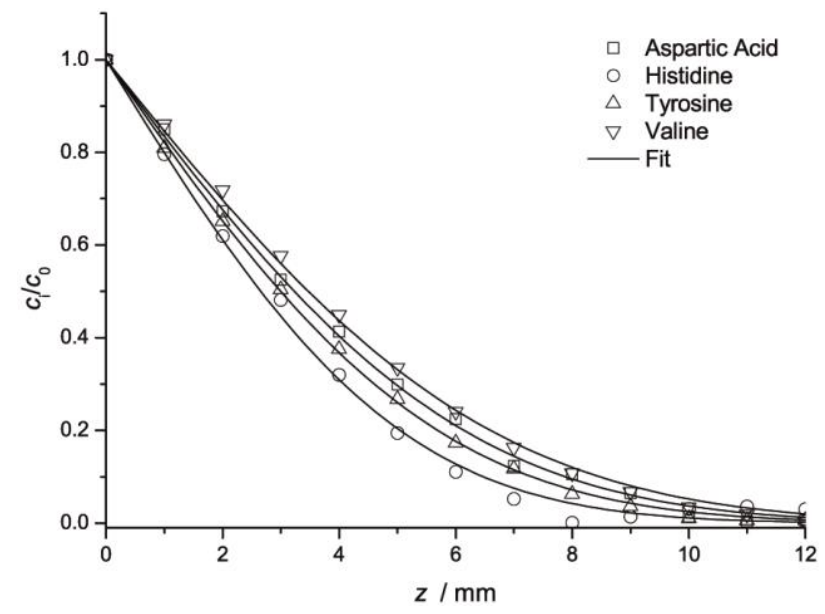

Figure 3-16: Concentration profile $c / c_{0}$ along slices $z$ for the amino acids mixture in the $3 \mathrm{wt} \%$ agar sample as obtained from spectra recorded $6 \mathrm{~h}$ after preparation of the sample. The fit is according to equation (3-3). 
These profiles were fitted to obtain diffusion coefficients $D_{\text {SSE }}$ via equation (3-3) which is the solution of Fick's $2^{\text {nd }}$ law $^{[120]}$ as obtained by Laplace transformation for the given boundary conditions. ${ }^{[121]}$

$$
\frac{c}{c_{o}}=\operatorname{erfc}\left(\frac{z}{2 \sqrt{D_{\mathrm{SSE}} t}}\right)
$$

Here, erfc is the complementary error-function and $t$ is the elapsed time since the diffusion into the gel has begun. The obtained values for three samples with increasing amount of agarose are summarized in Table 3-4.

Table 3-4: Average diffusion coefficients $\bar{D}_{\text {SSE }}$ and standard deviations for samples with $2 \mathrm{wt} \%$, $3 \mathrm{wt} \%$ and $5 \mathrm{wt} \%$ of agar as obtained through SSE. Values obtained after 2, 6 and $10 \mathrm{~h}$ were averaged.

\begin{tabular}{cccc}
\hline & $\bar{D}_{\text {SSE }}(2 \mathrm{wt} \%) / 10^{-10} \mathrm{~m}^{2} \mathrm{~s}^{-1}$ & $\bar{D}_{\text {SSE }}(3 \mathrm{wt} \%) / 10^{-10} \mathrm{~m}^{2} \mathrm{~s}^{-1}$ & $\bar{D}_{\text {SSE }}(5 \mathrm{wt} \%) / 10^{-10} \mathrm{~m}^{2} \mathrm{~s}^{-1}$ \\
\hline Asp & $7.0 \pm 0.5$ & $5.6 \pm 0.3$ & $5.2 \pm 0.7$ \\
His & $4.1 \pm 0.2$ & $3.6 \pm 0.2$ & $3.4 \pm 0.2$ \\
Tyr & $6.0 \pm 0.4$ & $4.7 \pm 0.1$ & $4.4 \pm 0.2$ \\
Val & $8.0 \pm 0.7$ & $6.4 \pm 0.4$ & $5.7 \pm 0.4$ \\
\hline
\end{tabular}

The obtained trend of diffusion coefficients is in accordance with the molecular weight of the observed amino acids ( $\mathrm{Val}<\mathrm{Asp}<\mathrm{His}<\mathrm{Tyr}$ ) yet interactions between amino acid and the gel certainly also induce effects that allow a tailoring of the chromatographic effect of agar: diffusion coefficients from the SSE approach differ sufficiently for all evaluated amino acids proving the anticipated chromatographic separation successful. For $2 \mathrm{wt} \%$ of agar, coefficients range from $4.1 \cdot 10^{-10} \mathrm{~m}^{2} / \mathrm{s}$ for L-His to $8.0 \cdot 10^{-10} \mathrm{~m}^{2} / \mathrm{s}$ for L-Val (maximum difference $\Delta_{\max } 3.9 \cdot 10^{-10} \mathrm{~m}^{2} / \mathrm{s}$ ). This gap is reduced for higher percentages of agar e.g. for $5 \mathrm{wt} \%$ corresponding values range from $3.4 \cdot 10^{-10}$ to $5.7 \cdot 10^{-10} \mathrm{~m}^{2} / \mathrm{s}\left(\Delta_{\max } 2.3 \cdot 10^{-10} \mathrm{~m}^{2} / \mathrm{s}\right)$. Hence, the separation of components can be adjusted through different amounts of agar.

In general, the obtained diffusion coefficients decrease with increasing agar weight percentage. Since higher amounts of agar should lead to higher gel densities and viscosities, this is in accordance with the Stokes-Einstein equation (diffusion coefficient is inversely proportional to viscosity). ${ }^{[32]}$ Additionally, there may be numerous further parameters, which influence the diffusion within the heterogeneous agar gel e.g. the order and type of crosslinking (recall that agar is a heterogeneous gel with strong inter polymer interaction in contrast to a homogeneous gel such as PS). ${ }^{[108 b]}$ This property was briefly examined in a small variational study on the relaxation behavior of molecules within the polymers by recording transverse relaxation times $T_{2}$ of $\mathrm{H}_{2} \mathrm{O}$ in samples of 2, 3 and $5 \mathrm{wt} \%$ agar with Carr-PurcellMeiboom-Gill (cpmg) pulse sequences ${ }^{[122]}$ (experimental details on sample preparation and parameters for the cpmg experiment are described in the experimental section 5.2). It is well-known that the pore size of (mostly inorganic) porous media (e.g. zeolites and metal organic frameworks) and the relaxation time (both longitudinal, $T_{1}$, and transverse, $T_{2}$ ) of probe molecules within the pores are correlated by equation (3-4). ${ }^{[123]}$

$$
\frac{1}{T_{2}}=\frac{1}{T_{2, \mathrm{bulk}}}+\delta \frac{S}{V}
$$


Here $T_{2, \text { bulk }}$ is the relaxation time of molecules in bulk solution (molecules in the center of a pore), $\delta$ is the surface relaxivity, $S$ is the surface area of the pore and $V$ is the volume of the pore. In the case of a spherical pore the equation is reduced to a linear dependence between $T_{2}$ and the pore radius. Hence, molecules in small pores exhibit shortened relaxation times. Figure 3-17 depicts how the signal intensities $I_{\text {cpmg }}$ decay during $t$ through transverse relaxation.

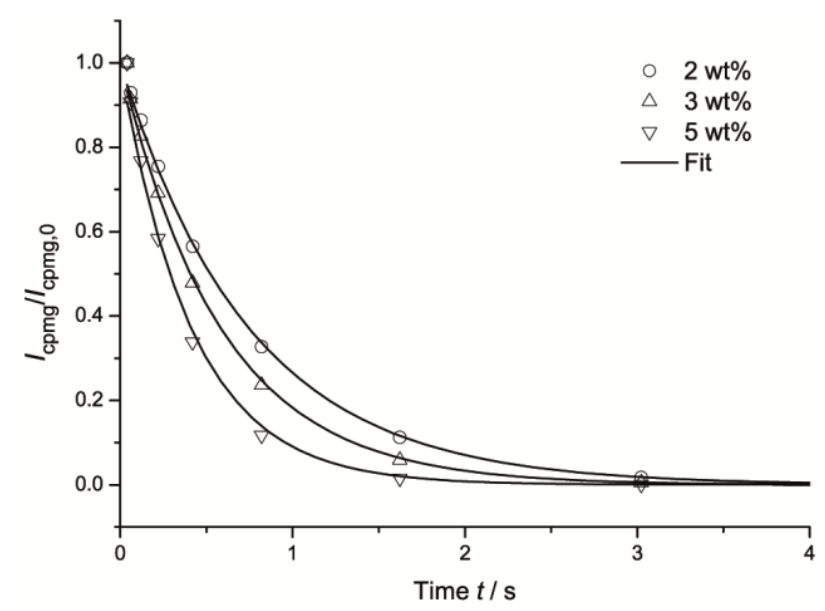

Figure 3-17: Integrals obtained from cpmg $T_{2}$ measurements of water in samples with $2 \mathrm{wt} \%$, $3 \mathrm{wt} \%$ and $5 \mathrm{wt} \%$ of agar versus elapsed time $t$ (details are described in the experimental section 5.2).

The decay has been fitted by the mono exponential equation (3-5) in which $I_{c p m g, 0}$ is the signal intensity at $t=0:[67]$

$$
\frac{I_{\text {cpmg }}}{I_{\text {cpmg }, 0}}=\exp \left(-\frac{t}{T_{2}}\right)
$$

Values are reported in Table 3-5.

Table 3-5: Transverse relaxation times $T_{2}$ for water protons in samples with $2 \mathrm{wt} \%$, $3 \mathrm{wt} \%$ and $5 \mathrm{wt} \%$ of agar as obtained through cpmg pulse sequences.

\begin{tabular}{cccc}
\hline & $2 \mathrm{wt} \%$ & $3 \mathrm{wt} \%$ & $5 \mathrm{wt} \%$ \\
\hline$T_{2}\left(\mathrm{H}_{2} \mathrm{O}\right) / \mathrm{s}$ & $0.75 \pm 0.02$ & $0.59 \pm 0.02$ & $0.41 \pm 0.02$ \\
\hline
\end{tabular}

The $T_{2}$ values steadily decrease with increasing agar weight percentage. Accordingly, the pore size decreases towards higher amounts of agarose which is equivalent to an increase in crosslinking. Yet, these relaxation times should be treated with care since equation (3-4) also indicates that the monoexponential decay as employed to fit the relaxation data could be insufficient. Within heterogeneous porous media a variety of pore sizes exist and the decay must be multi-exponential. This requires more sophisticated mathematical methods such as the inverse Laplace transformation to obtain relaxation time distributions. ${ }^{[124]}$ These methods have been successfully employed in the analysis of porous media and offer insights that may help for the characterization of polymeric gels and likewise. However, these methods also require extremely careful application are therefore beyond the scope of this work. ${ }^{[125]}$ 
A final remark on values listed in Table 3-4 must address the determination of $z_{0}$ and its implications. This procedure was not fully adapted to e.g. the concave gel surface which is negligible for the relative discrimination between various diffusing species, but the correct assignment of $z_{0}$ has a strong impact on the obtained absolute diffusion coefficients. For instance using $z_{1}=0$ (i.e. assigning the slice below $z_{0}$ as the boundary slice with $I / I_{0}=1$, vide supra) gives $\bar{D}_{\text {SSE }} 5.5 \cdot 10^{-10} \mathrm{~m}^{2} / \mathrm{s}$ for L-Asp acid in $2 \mathrm{wt} \%$ agar samples. This considerably deviates from $7 \cdot 0 \cdot 10^{-10} \mathrm{~m}^{2} / \mathrm{s}$ as listed in Table $3-4$. The literature value $D_{\mathrm{Lit}}$ of L-Asp acid in $\mathrm{H}_{2} \mathrm{O}$ is $7.4 \cdot 10^{-10} \mathrm{~m}^{2} / \mathrm{s}$ at a starting concentration of $0.030 \mathrm{~mol} / \mathrm{l}^{[126]}$ If it is taken into account that the diffusion coefficient decreases with increasing concentration ${ }^{[126]}$ (Lit.: $0.030 \mathrm{~mol} / \mathrm{l}$; SSE: $0.037 \mathrm{~mol} / \mathrm{l}$ ) and viscosity ${ }^{[32]}$ (Lit.: $\mathrm{H}_{2} \mathrm{O}$; SSE: $\mathrm{H}_{2} \mathrm{O}$ /agar gel matrix), the obtained values are eventually in remarkably good accordance with the literature values.

For the evaluation of the separation performance of the SSE approach, the results discussed above were compared to the matrix assisted DOSY technique. Therefore, standard DOSY spectra of each gel sample were recorded one week after preparation to deduce diffusion coefficients (experimental details on DOSY acquisition and processing are described in the experimental section 5.2 and 5.3). Results are listed in Table 3-6.

Table 3-6: Average diffusion coefficients $\bar{D}_{\text {DosY }}$ and standard deviations in units of $10^{-10} \mathrm{~m}^{2} / \mathrm{s}$ for samples with $2 \mathrm{wt} \%, 3 \mathrm{wt} \%$ and $5 \mathrm{wt} \%$ of agar as obtained through DOSY.

\begin{tabular}{cccc}
\hline & $D_{\text {DosY }}(2 \mathrm{wt} \%) / 10^{-10} \mathrm{~m}^{2} \mathrm{~s}^{-1}$ & $D_{\text {DosY }}(3 \mathrm{wt} \%) / 10^{-10} \mathrm{~m}^{2} \mathrm{~s}^{-1}$ & $D_{\text {DosY }}(5 \mathrm{wt} \%) / 10^{-10} \mathrm{~m}^{2} \mathrm{~s}^{-1}$ \\
\hline Asp & $4.3 \pm 0.2$ & $4.1 \pm 0.3$ & $3.7 \pm 0.4$ \\
His & $4.2 \pm 0.1$ & $4.4 \pm 0.2$ & $4.2 \pm 0.1$ \\
Tyr & $3.5 \pm 0.1$ & $3.6 \pm 0.1$ & $3.5 \pm 0.1$ \\
Val & $5.0 \pm 0.1$ & $4.9 \pm 0.1$ & $4.3 \pm 0.1$ \\
\hline
\end{tabular}

Absolute values $\bar{D}_{\text {SSE }}$ and $D_{\text {DOSY }}$ differ since the former describes chemical diffusion and the second tracer diffusion (which only become identical at infinite dilution). In contrast to SSE, the absolute values $D_{\text {Dosy }}$ are not reliable since $\mathrm{HDO} / \mathrm{H}_{2} \mathrm{O}$ in the homogeneous reference solution was determined to diffuse at a rate of $1.71 \cdot 10^{-9} \mathrm{~m}^{2} / \mathrm{s}$ (obtained through analysis of the solvent residual signal). This deviates strongly from the literature value of $D_{\mathrm{Lit}}=2.30 \cdot 10^{-9} \mathrm{~m}^{2} / \mathrm{s}^{\left[{ }^{[98 b]}\right.}$ Note, that the measurement of absolute diffusion coefficients via DOSY is a delicate task that depends on various experimental parameters (temperature, viscosity and likewise) and therefore, the majority of DOSY applications employ normalized/corrected diffusion coefficients that are obtained through the addition of reference substances. ${ }^{[34,36]}$ However, correction of the values listed in Table 3-4 with a factor obtained from the $\mathrm{HDO} / \mathrm{H}_{2} \mathrm{O}$ reference $\left(\mathrm{k}=D_{\mathrm{Lit}} / D_{\mathrm{DOSY}, \mathrm{Exp}} \approx 1.35, D_{\mathrm{DOSY}, \text { Cor }}=D_{\mathrm{DOSY}} \cdot k\right)$ does not lead to a higher compliance of values $\bar{D}_{\text {SSE }}$ and $D_{\text {DOSY,Cor }}$.

The essential conclusion drawn from these results is that the separation performance by $D_{\mathrm{DOSY}}$ is significantly worse than $\bar{D}_{\text {SSE. }}$. For example for $2 \mathrm{wt} \%$ of agar, coefficients range from $D_{\text {DOSY }} 3.5 \cdot 10^{-10} \mathrm{~m}^{2} / \mathrm{s}$ for L-Tyr to $5.0 \cdot 10^{-10} \mathrm{~m}^{2} / \mathrm{s}$ for L-Val. The corresponding difference $\Delta_{\max } 1.5 \cdot 10^{-10} \mathrm{~m}^{2} / \mathrm{s}$ is lower than for $\bar{D}_{\text {SSE }}\left(\Delta=2.0 \cdot 10^{-10} \mathrm{~m}^{2} / \mathrm{s}\right)$ and much lower than the biggest difference for $\bar{D}_{\text {SSE }}$ between L-His and L-Val as already pointed out above $\left(\Delta_{\max }=3.9 \cdot 10^{-10} \mathrm{~m}^{2} / \mathrm{s}\right)$. In contrast to SSE, values $D_{\text {DOsY }}$ for L-His and L-Asp acid $(2 \mathrm{wt} \%, 3 \mathrm{wt} \%)$ as well as L-histidine and L-Val $(5 \mathrm{wt} \%)$ are practically indistinguishable. 
Additionally, increasing weight percentage has a discernable impact on $D_{\text {DOSY }}$ of L-Asp acid and L-Val, while for L-His and L-Tyr no changes occur at all. This is remarkable since the latter amino acids share only very few characteristics. Elucidation of this phenomenon must be based on further investigations. In particular, molecular dynamics simulations have proven to be able to probe the causes of specific diffusivities. ${ }^{[127]}$

\subsection{Summary and Outlook Slice-Selective Excitation}

In the studies described above it could be shown that slice-selective NMR spectroscopy is an effective tool to perform

i) in situ observation of reactions,

ii) single-shot NMR titrations,

iii) qualitative NMR chromatography.

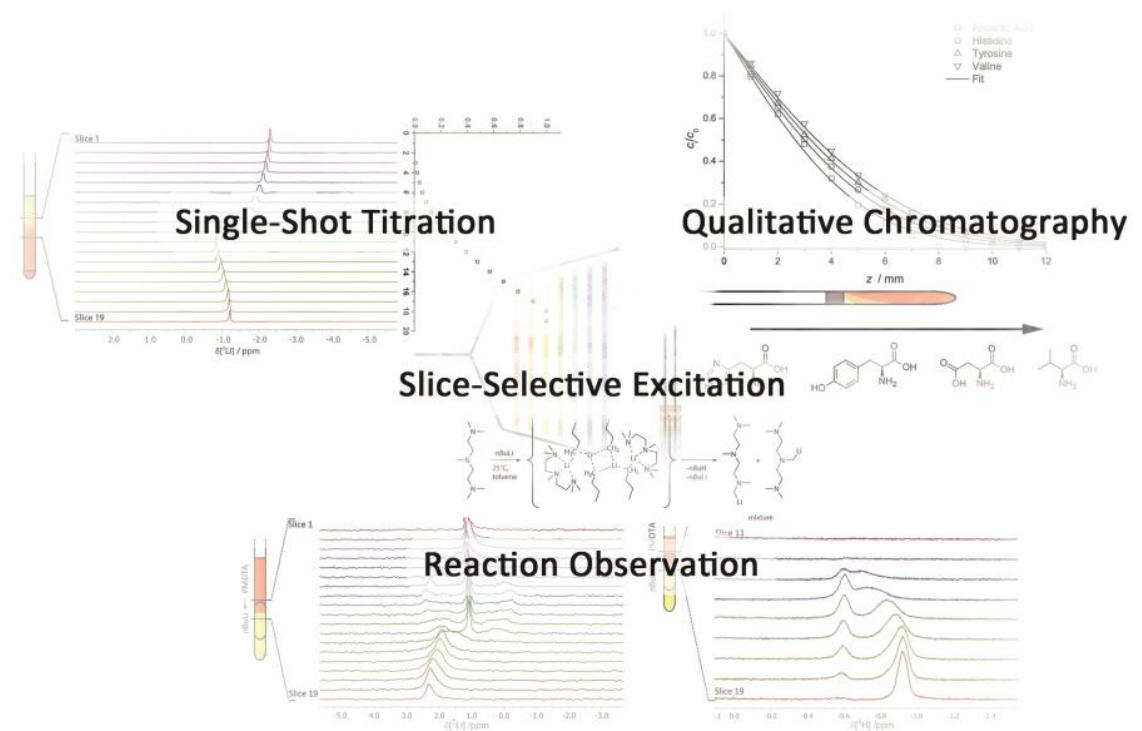

Figure 3-18: SSE on heterogeneous samples and its possible applications: i) In situ observation of reactions. ii) Single-shot NMR titration. iii) Qualitative NMR chromatography.

Reaction observation by slice-selective NMR spectroscopy was tested in the reaction between PMDTA and $n \mathrm{BuLi}$, where the previously characterized intermediate $\left[(n \mathrm{BuLi})_{2} \mathrm{PMDTA}\right]_{2}$ could be identified. ${ }^{[104]}$ A stretched PS gel was used as medium to slow down the reaction and avoid convection, immobilize one reactant $(n \mathrm{BuLi})$ with respect to the other (PMDTA) and principally enable the observation of anisotropic parameters as additional source of structural information. The magnitude of ${ }^{7} \mathrm{Li}$ RQCs can be obtained with ease from 1D SSE experiments and qualitative values have already proven to amend structure determination. Future developments should focus on the combination of SSE and 2D techniques such as the CLIP-HSQC to facilitate RDC structure determination (see section 4.2) of intermediates and derive the alignment which allows quantitative RQC analysis (see section 4.3). Additionally, the swelling process of PS gel as a more specific problem and future projects concerning this topic were addressed throughout the discussion. It is especially remarkable that the PS alignment 
strength shows a response at the reaction front between PMDTA and $n \mathrm{BuLi}$. This may result from the reaction heat from the lithiation and can be examined in future studies.

Single-shot titration was successfully tested in the complexation of a lithium salt by 12 -crown-4, where a conventional ${ }^{7} \mathrm{Li}$ chemical shift titration curve could be reproduced that indicates an initial strong coordination of $\mathrm{Li}^{+}$by one crown ether followed by a weaker coordination to give the double decker type complex. It has also been highlighted that this approach was greatly improved in subsequent studies by Mitrev et al. who utilized agar gel as diffusion controlling medium. ${ }^{[15]}$ Note that a further advantage of the gel method (both in titration and reaction monitoring applications) is the possibility to adjust the width of the reaction front and hence to zoom into the interesting region. Hence, it will be possible to apply slice-selective NMR titration to a broad range of reactions to obtain information about mechanisms as well as stoichiometry of complexes and products. Once again, this approach would greatly benefit from a combination of SSE with more elaborate 2D NMR techniques. For instance, DOSY and NOESY are powerful methods to characterize complexes. ${ }^{[24,26-27,33,36,50,62 \mathrm{~d}, 128]}$ Their (fast) SSE variants would significantly enhance the quantity and quality of information.

Qualitative chromatography was illustrated in a study with agar as stationary phase and the analytes LAsp acid, L-His, L-Tyr and L-Val. It was demonstrated that in this particular case the discrimination through SSE is superior to DOSY. ${ }^{[18 \mathrm{a}, 118 \mathrm{~b}, 118 \mathrm{dd}]}$ Therefore, the SSE approach to NMR chromatography is at least complementary to the established DOSY method and should be considered in cases where DOSY gives ambiguous results. Different amounts of agar gel were evaluated as stationary media and their separation behavior was attributed to pore sizes and the amount of crosslinking (through physical entanglement as well as electrostatic interactions). Support for these hypotheses was found by s small variational study on $T_{2}$ relaxation data. Relaxation data were obtained by standard cpmg pulse sequences and in general seem to be valuable observables for the examination of swollen polymeric gels. Future applications could determine the extent to which alignment parameters and relaxation rates are correlated. Diffusion measurements (both through SSE and DOSY) will amend this approach as well. In general, combination of SSE chromatography and 2D NMR techniques can serve once more as a basic but powerful method for the characterization of mixtures. Note that the use of chiral agarose, as of other chiral alignment media, principally enables the separation of racemic mixtures. ${ }^{[129]}$

Here, further studies could aim at the evaluation of this approach in combination with molecular dynamics simulations of diffusion to enable full enantiomer discrimination. The methods shown above will add further impetus to research in slice-selective NMR spectroscopy of heterogeneous samples in particular polymeric gels. 


\section{ANISOTROPIC NMR SPECTROSCOPY IN POLYMERIC GELS}

\subsection{Alignment of Small Symmetric Molecules}

Weak alignment of molecules for NMR experiments is nowadays achieved by a wide range of anisotropic media. The most common media are nematic liquid crystals ${ }^{[130]}$ and mechanically deformed gels. ${ }^{[70,91]}$ Originally, research started within liquid crystals and these systems have been studied very thoroughly ever since. Today, comprehensive theoretical treatment of nematic liquid crystals offers unprecedented insights and enables a comprehensive interpretation of alignment phenomena within these media. ${ }^{[80,131]}$

Theoretical estimations of alignment has been achieved through steric models ${ }^{[81]}$ and first principle approximations on inertia ${ }^{[79 e]}$ or gyration ${ }^{[79 e]}$ of molecules. These models sufficiently predict the alignment of large molecules and are therefore commonly incorporated in RDC analysis programs. ${ }^{[81,83]}$ Meanwhile, for small molecules only very little is known about the mechanism of alignment. ${ }^{[132]}$ It is obvious that e.g. the influence of sparse electrostatic interactions must be reviewed in the case of small molecules to supplement purely geometric approaches. In this thesis, the weak alignment of benzene derivatives and polycyclic homologues within crosslinked stretched PS and polybutylacrylate (PBA) was examined to obtain an improved understanding of small molecule alignment in polymer gels (Scheme 4-1).

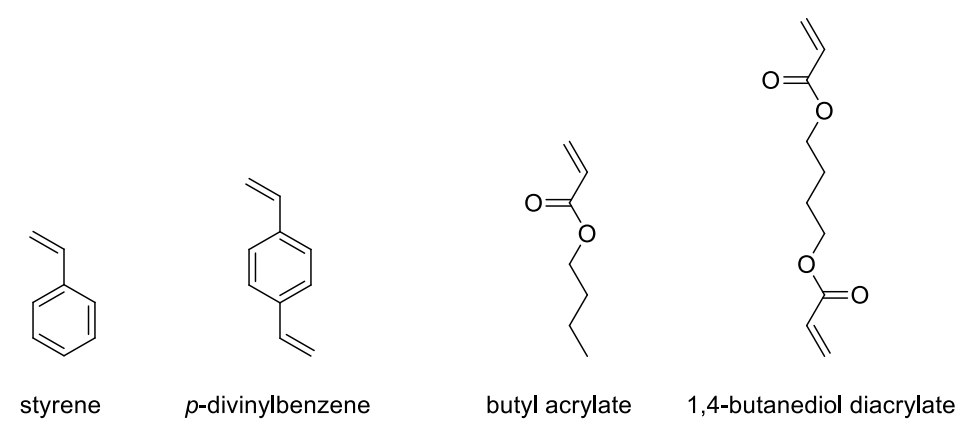

Scheme 4-1: (Atactic) PS is thermally polymerized from styrene with covalent crosslinking between PS polymer strands through divinylbenzene (technical grade: 80\% p-divinylbenzene). For (atactic) PBA, thermal polymerization of butyl acrylate and 1,4-butanediol diacrylate as cross-linker is performed.

In particular, the alignment of toluene, benzoic acid, methyl benzoate, 4-methylphenol and naphthalene in dichloromethane (DCM)/PS and DCM/PBA were evaluated. First ${ }^{1} J_{\mathrm{CH}}$ couplings were measured for each molecule by CLIP-HSQC experiments of isotropic samples (in neat DCM). Subsequently, anisotropic samples of these substances in PS and PBA were prepared. Swelling and sufficient equilibration of PS and PBA in DCM was achieved after four and three days, respectively. ${ }^{[75 b]}$ Further CLIP-HSQC measurements yielded couplings ${ }^{1} T_{\mathrm{CH}}$ from which one-bond RDCs were calculated $\left({ }^{1} D_{\mathrm{CH}}={ }^{1} T_{\mathrm{CH}}-J_{\mathrm{CH}}\right)$. Details on sample preparation and CLIP-HSQC parameters are described in the experimental sections 5.1, 5.2 and 5.3. The molecular geometry can be conveniently described by polar 
coordinates. Therefore, the molecules were set into coordinate systems by aligning their $C_{2}$ axes (all functional groups are considered to rapidly rotate as to fulfill this symmetry) along $z$. Naphthalene was aligned along $z$ by its long $C_{2}$ axis. Furthermore, the ring planes were embedded within the $y$ - $z$ plane. Polar angles $\vartheta$ were then determined between the $z$ axis and the respective $\mathrm{C}-\mathrm{H}\left(\mathrm{C}-\mathrm{CH}_{3}\right)$ bond vector (Figure 4-1).

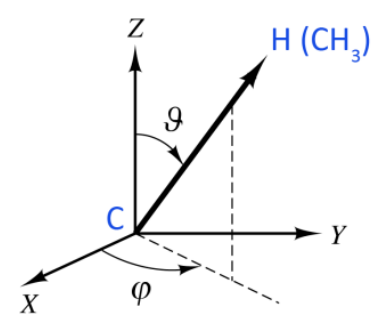

Figure 4-1: Polar angle $\vartheta$ and azimuthal angle $\varphi$ for an arbitrary $\mathrm{C}-\mathrm{H}$ bond vector.

The parallel arrangement between the ring and the $y$ - $z$ plane leads to $\varphi=90^{\circ}$ for all bonds. RDCs and associated distances and angles are summarized in Table 4-1.

Table 4-1: Textbook C-H Distances $R_{\mathrm{IS}}$ and polar angles 9 between the $z$ axis (green arrow) and the particular $\mathrm{C}-\mathrm{H}\left(\mathrm{C}-\mathrm{CH}_{3}\right)$ vectors (note that azimuthal angles are $90^{\circ}$ since the ring is embedded within the $y-z$ plane; red and green arrows). Experimental RDCs for the corresponding bonds of structures aligned in PS/DCM as well as $\mathrm{PBA} / \mathrm{DCM}$.

\begin{tabular}{|c|c|c|c|c|c|}
\hline & & $R_{\mathrm{IS}} / \AA$ & $9 /^{\circ}$ & ${ }^{1} D_{\mathrm{CH}}(\mathrm{PS}) / \mathrm{Hz}$ & ${ }^{1} D_{\mathrm{CH}}(\mathrm{PBA}) / \mathrm{Hz}$ \\
\hline \multirow{4}{*}{ Toluene } & $1-\mathrm{CH}_{3}$ & 1.09 & $0^{\text {a }}$ & $2.5 \pm 0.2^{\mathrm{a}}$ & $7.2 \pm 0.9^{\mathrm{a}}$ \\
\hline & $\mathrm{C} 2-\mathrm{H} 2$ & 1.09 & 60 & $-25.1 \pm 1.5$ & $-9.1 \pm 0.6$ \\
\hline & $\mathrm{C} 3-\mathrm{H} 3$ & 1.09 & 120 & $-23.6 \pm 0.7$ & $-10.6 \pm 0.3$ \\
\hline & $\mathrm{C} 4-\mathrm{H} 4$ & 1.09 & 180 & $-10.4 \pm 0.6$ & $-19.7 \pm 0.3$ \\
\hline \multirow{3}{*}{ Benzoic acid } & $\mathrm{C} 2-\mathrm{H} 2$ & 1.09 & 60 & $-18.1 \pm 1.4$ & $-5.8 \pm 0.4$ \\
\hline & $\mathrm{C} 3-\mathrm{H} 3$ & 1.09 & 120 & $-18.9 \pm 0.6$ & $-8.3 \pm 0.5$ \\
\hline & $\mathrm{C} 4-\mathrm{H} 4$ & 1.09 & 180 & $-42.7 \pm 0.9$ & $25.2 \pm 0.6$ \\
\hline \multirow{3}{*}{ Methyl benzoate } & $\mathrm{C} 2-\mathrm{H} 2$ & 1.09 & 60 & $-30.4 \pm 0.5$ & $-9.6 \pm 0.2$ \\
\hline & $\mathrm{C} 3-\mathrm{H} 3$ & 1.09 & 120 & $-29.2 \pm 1.6$ & $-9.9 \pm 0.7$ \\
\hline & $\mathrm{C} 4-\mathrm{H} 4$ & 1.09 & 180 & $-42.4 \pm 0.8$ & $-21.8 \pm 1.7$ \\
\hline \multirow{3}{*}{ 4-Methylphenol } & $\mathrm{C} 2-\mathrm{H} 2$ & 1.09 & 60 & $-31.2 \pm 0.7$ & $13.1 \pm 0.8$ \\
\hline & $\mathrm{C} 3-\mathrm{H} 3$ & 1.09 & 120 & $-31.7 \pm 3.6$ & $13.8 \pm 0.7$ \\
\hline & $4-\mathrm{CH}_{3}$ & 1.09 & $180^{\mathrm{a}}$ & $2.6 \pm 0.6^{\mathrm{a}}$ & $0.4 \pm 0.1^{\mathrm{a}}$ \\
\hline \multirow{2}{*}{ Naphthalene } & $\mathrm{C} 1-\mathrm{H} 1$ & 1.09 & $90^{c}$ & $-51.7 \pm 0.4$ & $-8.8 \pm 1.7$ \\
\hline & $\mathrm{C} 2-\mathrm{H} 2$ & 1.09 & $30^{c}$ & $-22.5 \pm 1.2$ & $-30.9 \pm 1.2$ \\
\hline
\end{tabular}

${ }^{\text {a }}$ RDCs for rotating methyl groups can be treated as ${ }^{1} \mathrm{D}_{\mathrm{CH}}$ with a corresponding vector along the $C_{3}$ symmetry axis of the methyl group by scaling the original value with -3 (e.g. toluene $\mathrm{C} 1-\mathrm{Me}$ then yields $-7.5 \mathrm{~Hz}$ in PS). ${ }^{[133]}$

The investigated molecules were treated like textbook structures with aromatic $\mathrm{C}-\mathrm{H}$ distances of $1.09 \AA$ and prevalent $30^{\circ}, 60^{\circ}, 90^{\circ}, 120^{\circ}$ and $180^{\circ}$ angles between bonds concerning the aromatic moiety. Note 
that the averaged RDCs which are measured for a methyl group can be easily transformed into a virtual $\mathrm{C}-\mathrm{H}$ RDC pointing along the $\mathrm{C}-\mathrm{CH}_{3}$ threefold symmetry axis by applying a scaling factor of -3 . This factor stems from the more general formula (4-1) which is valid for a freely rotating group leading to averaged RDCs.

$$
\langle D\rangle=D_{\|} \frac{3 \cos ^{2} \phi-1}{2}
$$

Here $\langle D\rangle$ represents the averaged RDC, $D_{\|}$would be the RDC of a virtual C-H bond pointing along the axis of the rotation and $\phi$ is the angle between this axis and each rotating vector of the methyl group. In the case of ideally tetrahedral angles $\mathrm{H}-\mathrm{C}-\mathrm{H}$ and $\mathrm{C}-\mathrm{C}-\mathrm{H}$ within the methyl group, $\phi$ is $109.5^{\circ}$ and the (inverse) scaling factor becomes $-1 / 3$. Usually, angles are non-ideal and hence the scaling factor is different from $-1 / 3$. This becomes visible if collinear $\mathrm{C}-\mathrm{H}$ vectors exist which then display couplings that are beyond the virtual RDC as in the case of toluene (vide infra). In fact, these considerations have allowed the investigation of subtle differences of angles in methyl as well as methylene groups. ${ }^{[133-134]}$ In the cases shown above, the methyl RDC for toluene was dismissed, since the collinear RDC for C4-H4 is much better determined. However, for 4-methylphenol the obtained methyl RDC is crucial and must be included for further investigations.

The coordinate system chosen above is very convenient, since its axes coincide with the molecular symmetry elements. Likewise, the PAS of the alignment tensor $\mathbf{A}_{\mathrm{PAS}}$ must contain existing symmetry elements. This means that the axes of the chosen coordinate system also span the PAS of the alignment tensor, i.e. the alignment tensor is diagonalized: ${ }^{[64,135]}$

$$
\mathbf{A}_{\mathrm{PAS}}=\left(\begin{array}{ccc}
A_{\tilde{x}} & 0 & 0 \\
0 & A_{\tilde{y}} & 0 \\
0 & 0 & A_{\tilde{z}}
\end{array}\right)
$$

Thus, equation (1-7) can be transformed into polar coordinates and rewritten:

$$
D=k\left(A_{\tilde{\mathrm{X}}} \sin ^{2} \vartheta \cos ^{2} \varphi+A_{\widetilde{\mathrm{Y}}} \sin ^{2} \vartheta \sin ^{2} \varphi+A_{\tilde{\mathrm{Z}}} \cos ^{2} \vartheta\right)
$$

For each molecule, the measured RDCs and corresponding angles and distances can then be employed to constitute a linear equation system to which the $A_{\tilde{\mathrm{x}}}+A_{\tilde{\mathrm{y}}}+A_{\tilde{z}}=0$ can be added. Therefore, only two independent alignment parameters remain. These essential effects of molecular symmetry on the number of independent alignment tensor elements are additionally summarized in Table 4-2:[135] 
Table 4-2: Independent elements of the alignment tensor for various point groups.

\begin{tabular}{ll}
\hline Point group & Independent alignment tensor elements \\
\hline$C_{1}, C_{\mathrm{i}}$ & $A_{z z}, A_{\mathrm{xy}}, A_{\mathrm{xz}}, A_{y y}, A_{\mathrm{yz}}(=5)^{\mathrm{a}}$ \\
$C_{2}, C_{2 \mathrm{~h}}, C_{\mathrm{s}}$ & $A_{z z}, A_{\mathrm{yy}}, A_{\mathrm{xy}}(=3)^{\mathrm{a}}$ \\
$C_{2 \mathrm{v}}, D_{2}, D_{2 \mathrm{~h}}$ & $A_{z z}, A_{\mathrm{yy}}(=2)^{\mathrm{a}}$ \\
$C_{>2}, C_{>2 \mathrm{v}}, C_{>2 \mathrm{~h}}, D_{>2}, D_{>2 \mathrm{~h}}, D_{\geq 2 \mathrm{~d}}$ & $A_{z z}(=1)^{\mathrm{a}}$ \\
$T, T_{\mathrm{d}}, O, O_{\mathrm{h}}, K_{\mathrm{h}}$ & - \\
\hline
\end{tabular}

${ }^{a}$ Note the convention $\left|A_{\tilde{x}}\right| \leq\left|A_{\tilde{y}}\right| \leq\left|A_{\tilde{z}}\right|$.

In general, at least five independent RDCs are necessary to derive an alignment tensor for molecules of arbitrary symmetry. In this work, dependent relates to two RDCs for parallel internuclear vectors or any more than three vectors located in one plane. According to Table 4-1, the number of independent RDCs exceeds the necessary independent alignment tensor elements for the observed molecules (e.g. toluene $C_{2 \mathrm{v}}$ : two tensor elements, three independent experimental RDCs). These overdetermined equation systems can be solved by the least-square routine numpy.linalg.lstsq as included in the NumPy package for Python (script is added to the Appendix). ${ }^{[136]}$ Resulting alignment tensors for PS are displayed in Table 4-3.

Table 4-3: Alignment tensor elements $A_{\tilde{\mathbf{x}}}, A_{\tilde{\mathbf{y}}}$ and $A_{\tilde{\mathbf{z}}}$ for the set of molecules in swollen DCM/PS as determined by least-square techniques from overdetermined equation systems of (4-3) with values taken from Table 4-1. Additionally, the magnitude of these values and the orientation within the molecular frame are represented by arrows with corresponding length, their sign is represented by their color (blue: positive, red: negative).

\begin{tabular}{llllll} 
& Toluene & Benzoic acid & Methyl benzoate & 4-Methylphenol \\
\hline$A_{\tilde{\mathbf{x}}} / 10^{-4}$ & 1.4 & &
\end{tabular}

Note, that solutions for linear equation systems of (4-3) were renamed according to the convention $\left|A_{\tilde{\mathrm{x}}}\right| \leq\left|A_{\tilde{\mathrm{y}}}\right| \leq\left|A_{\tilde{\mathrm{z}}}\right|{ }^{[64]}$ Additionally, figures of the molecules are shown in which arrows indicate the magnitude and sign of the alignment tensor elements through length and color, respectively. Recall that the alignment tensor essentially describes the probability to find one of its principal axes oriented along the magnetic field relative to the probability in isotropic solution. A more positive principal alignment tensor element implies a higher probability to find this axis oriented along the magnetic field vector. In Table 4-3 molecules are depicted in the (exaggeratedly) preferred orientation of the molecule within the laboratory frame, i.e. with their highest positive alignment tensor element oriented along the magnetic field vector (vertical sheet axis). Hence, the orientation of toluene (i.e. its $C_{2}$ axis) in PS is found perpendicular or orthogonal to the magnetic field. Toluene is then also perpendicularly oriented towards the elongated pores within the gel that result from the swelling of the polymer. This is remarkable since these pores are usually assumed to be responsible for the alignment of the solutes according to simple 
sterical/geometrical considerations. 4-methylphenol and naphthalene show an analogous mismatch between PS pore geometry and molecular shape.

Benzoic acid and methyl benzoate behave differently and show the allegedly steric orientation which will be referred to as parallel or aligned along the field/pore. Discussion of absolute alignment values is omitted, since RQCs of $\mathrm{CD}_{2} \mathrm{Cl}_{2}$ were not fully consistent for the five samples, indicating different alignment strengths. Hence, a purely qualitative classification is made. It can be assumed that the sum of i) generalized steric (repulsive, parallel) and ii) directional (attractive, orthogonal) interaromatic forces defines the alignment characteristics of these small aromatic molecules in PS. For toluene, 4methylphenol and naphthalene the directional interaromatic forces prevail (Figure 4-2, green).
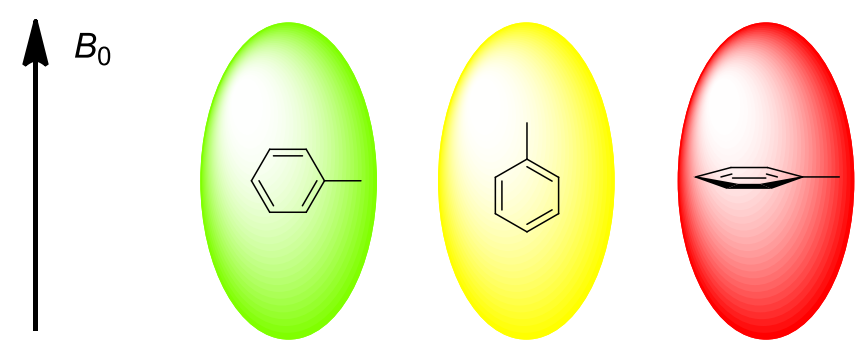

Figure 4-2: Schematic illustration of the most probable (green), less probable (yellow) and least probable orientation of toluene in PS.

Interactions between aromatic compounds are influenced by a wide range of factors that include electrostatic, hydrophobic and van-der-Waals interactions. ${ }^{[137]}$ Attribution of these factors to the situation encountered in the gel-solute mixture of small aromatic molecules and PS ring moieties requires very elaborate and extensive studies that are beyond the scope of this thesis. In particular, the orientation of the polymer ring moieties is not ascertainable by solution state NMR spectroscopic means. Therefore, common structural motifs like the stacked (also face-to-face or sandwich), offset stacked or T-shaped (also edge-to-face) configuration of aromatic rings, which in turn can be attributed to certain interactions, are not traceable. ${ }^{[137]}$

The sterical parallel force becomes clear from the second and least probable orientation (Figure 4-2 yellow and red, respectively). The orthogonal alignment of the ring plane towards the magnetic field (long pore axis) is never preferred, as the associated tensor element $A_{\tilde{Z}}$ is always the most negative tensor element (also see Table 4-3).

In contrast to toluene, in 4-methylphenol and naphthalene the parallel orientation of benzoic acid may be traced back to the formation of long hydrogen-bonded dimers which might overcome a tipping point at which steric demand exceeds the influence of interaromatic orthogonal forces. This hypothesis is limited since methyl benzoate obviously cannot form hydrogen-bonded dimers but is also in parallel orientation to the pore. However, instead of increasing the spatial extension (dimerization), reduction of the additional interaromatic attractive force may also lead to a prevalent parallel alignment, as is the situation for methyl benzoate: one difference between the molecules grouped into the parallel and orthogonal categories is the inductive effect of the substituents and the resulting electronic characteristics. The carboxylic acid and its ester are characterized by a $-\mathrm{M}$ (mesomeric) and $-\mathrm{I}$ (inductive) effect, whereas the other aromats are associated with $\mathrm{a}+\mathrm{M}$ and $+\mathrm{I}$ or no effect. This can be 
illustrated by electrostatic potential (ESP) maps, which were exemplary obtained from B3LYP/ 6$31 \mathrm{G}(\mathrm{d}, \mathrm{p})$ geometry optimization and subsequent analogous DFT calculations and the CHELPG method $^{[138]}$ for toluene and benzoic acid. These surfaces are depicted in Figure 4-3.
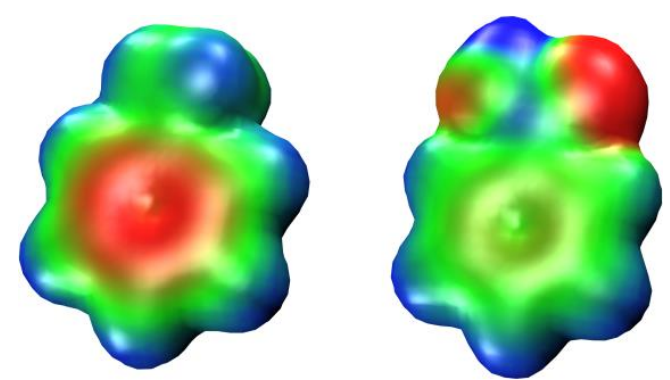

Figure 4-3: ESP maps for toluene (left) and benzoic acid (right) as derived from B3LYP/6-31G(d,p) DFT calculations and the CHELPG method. Red marks areas of high electrostatic potentials (more negative charge), blue corresponds to low electrostatic potentials (less negative charge).

The low electrostatic potential within the ring moiety may signal a decreased interaromatic interaction in the case of benzoic acid and methyl benzoate which allows domination of the steric forces that lead to parallel orientation.

In summary, only vague molecular features have been found that delimit the parallel from the orthogonal orientation: it is very likely that for small molecules the type and strength of (attractive) intermolecular interactions and more generalized steric (repulsive electrostatic) interaction are both responsible for alignment media phenomena. This hypothesis is supported by more distinct results for the orientation of aromats in PBA. The corresponding alignment tensor elements are summarized in Table 4-4.

Table 4-4: Alignment tensor elements $A_{\tilde{x}}, A_{\tilde{y}}$ and $A_{\tilde{Z}}$ for a set of molecules in swollen DCM/PBA as determined by SVD from overdetermined equation systems of (4-3) with values taken from Table 4-1. Additionally, the magnitude of these values and the orientation within the molecular frame are represented by arrows with corresponding length, their sign is represented by their color (blue: positive, red: negative).

\begin{tabular}{l|l|l|l|l|l}
\hline & Toluene & \multicolumn{1}{|c|}{ Benzoic acid } & Methyl benzoate & 4-Methylphenol & Naphthalene \\
\hline $\boldsymbol{A}_{\tilde{\mathbf{x}}} / 10^{-4}$ & 0.9 & 1.1 & \\
$\boldsymbol{A}_{\tilde{\mathbf{y}}} / 10^{-4}$ & 2.8
\end{tabular}

In PBA, the principal values of the alignment tensor for toluene, methyl benzoate and naphthalene indicate parallel orientation in agreement with steric considerations. In contrast, benzoic acid once more behaves differently and orients perpendicular towards the magnetic field as can also be observed to some extend for 4-methylphenol which has a very unique orientation. These latter compounds have the possibility to establish hydrogen bonds towards the ester group of PBA. Hence, the orientation of small molecules in PBA can be strictly derived from either a sterical match between pore and molecular shape (toluene, methyl benzoate, naphthalene), or from the molecule's ability to form hydrogen bonds 
with the polymer (benzoic acid and 4-methylphenol). The results on small molecules alignment in polymeric gels have implications for applications of RDCs and RQCs in structure determination:

i) A priori prediction of alignment based on simple sterical/mechanical models is often not sufficient for small molecules.

ii) The interplay of attractive and repulsive interactions changes the energetic states of solutes within the alignment medium, similar to strong solvent effects. Hence, the population of conformers can be considerably biased compared to isotropic solutions.

The prediction of alignment will be addressed in the following subsection. The effect of conformation will be briefly discussed in section 4.2 .

\subsubsection{Molecular Dynamics Simulations}

Starting from a proposed molecular structure one can either back-calculate the alignment tensor from experimental RDCs (vide supra) or estimate theoretical alignment from first principles such as the moment of inertia (Tramite) ${ }^{[7]}$ or the model's shape combined with steric obstruction (PALES). ${ }^{[81]}$ Prediction of RDCs by the latter means is successfully performed for larger biomolecules, but the results introduced above underline their limits in the case of small to medium sized molecules. In the last few years, some effort has been put into the study of the alignment of small organic solutes in liquid crystals by stochastic molecular dynamics (MD) simulations ${ }^{[80,131]}$, but only very recently attempts were made to transfer this knowledge and simulate solutes in stretched polymeric gels. In an innovative study Frank et al. directly obtained averaged RDCs from trajectories of strychnine in the presence of an elongated PS strand. ${ }^{[132]}$ However, this approach must be followed by further benchmark studies to learn more about its potentials and limitations. Toluene and benzoic acid exhibited unusual, diverse alignment in the experiments described above and were therefore chosen to be evaluated in MD simulations of solute polymer systems within this thesis.

MD simulations were performed with GROMACS 5.1.2. ${ }^{[139]}$ The topology was built with the optimized potentials for liquid simulations/all-atom (OPLS) and tabulated standard OPLS charges (exemplary topology files are included in the Appendix). ${ }^{[140]}$ Similar to the original publication by Frank et al., polymeric PS and PBA strands were mimicked by six atacic styrene and butyl acrylate units, respectively. First attempts revealed that the phenyl moiety at the methylene end of the strand was strongly bent away during MD runs (observed by spatial distribution functions, vide infra). Hence, in this work methyl groups were added to the methylene backbone end which gave a consistent orientation of the phenyl moieties. The strands were positionally restrained by adding strong isotropic forces on the methyl end groups. A typical energy optimized structure for PBA and benzoic acid prior to the start of the simulation is shown in Figure 4-4. 


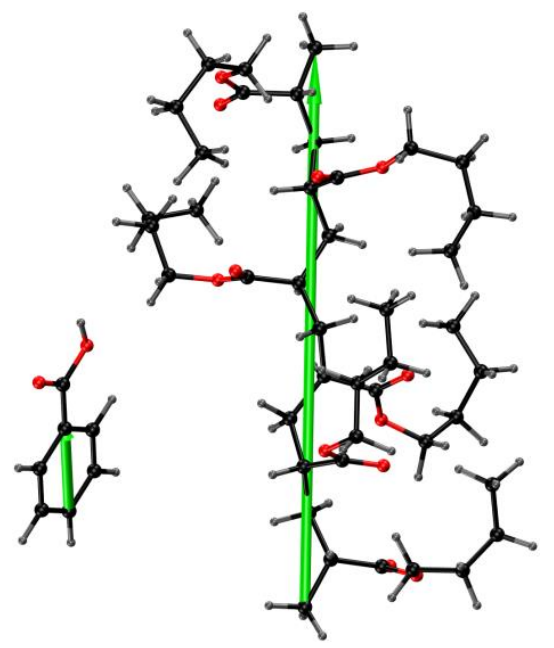

Figure 4-4: Energy-optimized starting position for the PBA strand and a benzoic acid molecule prior to the simulation. The green polymer strand arrow connects both positionally fixed methyl groups. The benzoic acid is characterized by an arrow parallel to the quasi $C_{2}(\mathrm{C} 1-\mathrm{C} 4)$ axis.

MD runs were performed in small cubic boxes with periodic boundary conditions and further options as suggested by Frank et al. Most notably, no solvent molecules were added, since the authors reported that only then alignment became non-zero during the production runs. To mimic solvent effects, Langevin dynamics were applied in which friction terms were added. Analogous to the original proceedings, long $400 \mathrm{~ns}$ production runs were executed directly after energy minimization. The Berendsen thermostat ${ }^{[141]}$ was set to a target temperature of $300 \mathrm{~K}$ but no preceding thermal equilibration run was conducted. ${ }^{a}$ Further details on the MD simulation protocol as well as analyzing tools are described in the experimental section (5.4). The production runs were analyzed by sampling $\cos ^{2} \theta-1 / 3$ where $\theta$ is the polar angle between $\mathrm{C}-\mathrm{H}$ vectors and the vector defined by the two positionally restrained methyl carbons. This term was averaged over time to give $\left\langle\cos ^{2} \theta-1 / 3\right\rangle$. The progression of this averaging for toluene and benzoic acid in PS is depicted in Figure 4-5.
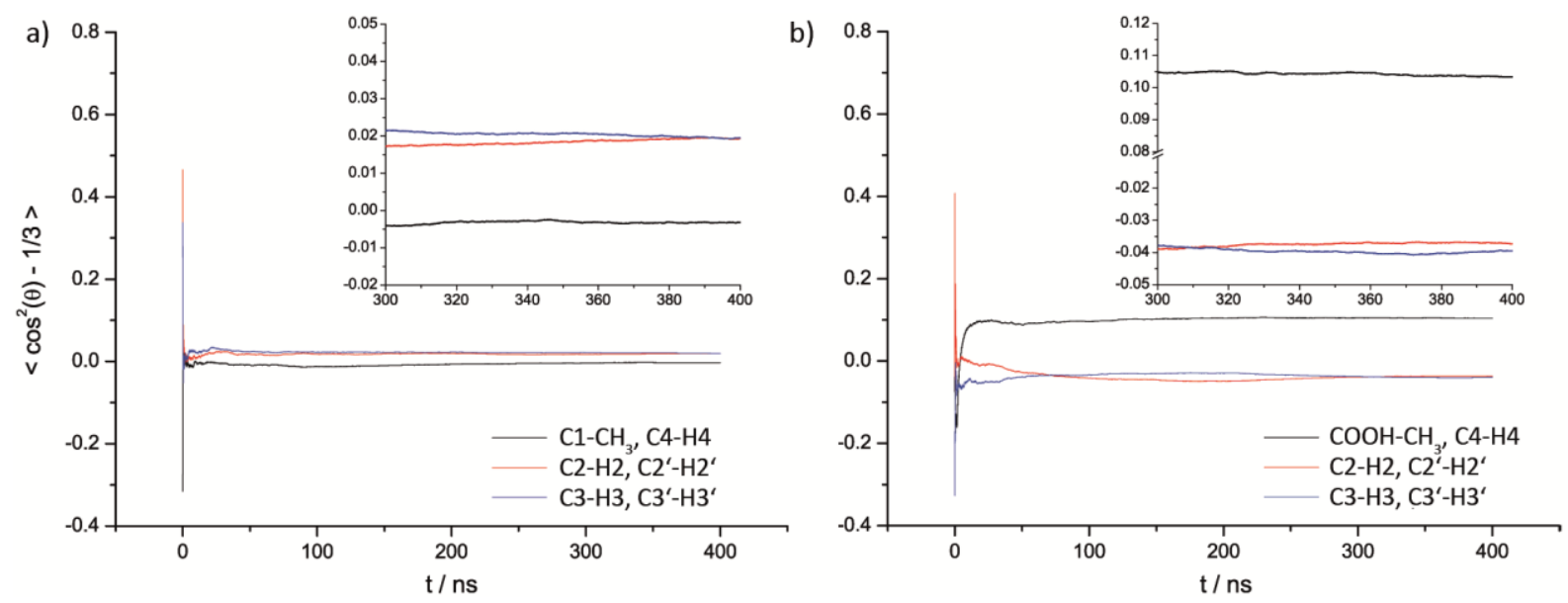

Figure 4-5: Convergence of the averaged angle term $\left\langle\cos ^{2} \theta-1 / 3\right\rangle$ between $\mathrm{C}-\mathrm{H}$ vectors for a) toluene and b) benzoic acid and the vector connecting the fixed methyl groups of the PS polymer strand backbone.

\footnotetext{
a Pressure equilibration is not feasible due to the positional restraints. The extremely long duration of the production run covers up short thermal non-equilibrium contributions at the beginning of the run.
} 
Figure 4-5 a) summarizes the averaged orientation of toluene in PS. Orientational equilibration, which is signaled by constant values $\left\langle\cos ^{2} \theta-1 / 3\right\rangle$, is reached after about $100 \mathrm{~ns}$. The angle between the polymer strand and $\mathrm{C} 4-\mathrm{H} 4\left(\mathrm{C} 1-\mathrm{CH}_{3}\right)$ shows an overall negative value for $\left\langle\cos ^{2} \theta-1 / 3\right\rangle$. For $\mathrm{C} 2-\mathrm{H} 2$ and $\mathrm{C} 3-\mathrm{H} 3$ positive values were obtained. This relationship is inversed for benzoic acid in Figure 4-5 b). Since more positive values for $\left\langle\cos ^{2} \theta-1 / 3\right\rangle$ correspond to a more parallel orientation of the corresponding vectors and more negative values indicate predominantly orthogonal orientations (also see Figure 1-11 and corresponding equation (1-3)), these simulations qualitatively reproduce the experimental findings. As MD simulations are based on a set of classical forces that include electrostatic attraction and (steric) repulsion, the rationalization of orientation through shape and electronic effects is further corroborated. However, note that the utilization of standard OPLS charges implies that benzoic acid and toluene are only distinguished by the charges of their substituents while the ring charges are the same. ${ }^{a}$ Despite this deficiency, divergent experimental results were reproduced. This hints that interactions between the polymer and $\mathrm{COOH} / \mathrm{CH}_{3}$ play a critical role. The analogous treatment of both compounds in PBA yields further insights. Interestingly, these MD simulations first resulted in very weak (benzoic acid) or no (toluene) alignment. After reproducing these observations with different starting geometries, the MD simulation protocol was slightly varied by incorporating 50 DCM molecules. ${ }^{\text {b }}$ This gave pronounced orientations for benzoic acid in PBA as depicted in Figure 4-6.
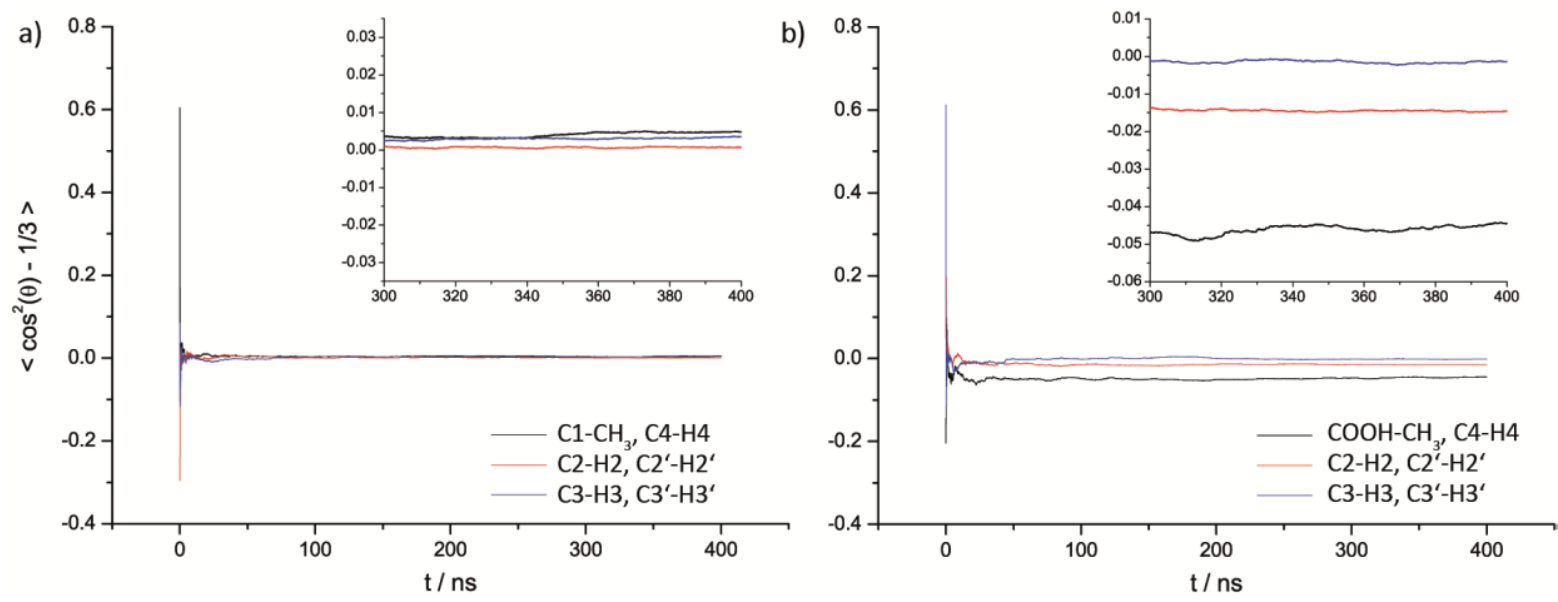

Figure 4-6: Convergence of the averaged angle term $\left\langle\cos ^{2} \theta-1 / 3\right\rangle$ between $\mathrm{C}-\mathrm{H}$ vectors for a) toluene and b) benzoic acid and the vector connecting the fixed methyl group of the PBA polymer strand backbone. The simulation additionally included 50 DCM molecules.

Toluene is still not noticeably ordered within PBA but the orientation of benzoic acid is in accordance with experimental results. The vector $\mathrm{C} 4-\mathrm{H} 4$ exhibits the most negative values for $\left\langle\cos ^{2} \theta-1 / 3\right\rangle$ which corresponds to a predominantly orthogonal orientation between the molecule and the polymer strand.

\footnotetext{
${ }^{a}$ Negative carbon and positive hydrogen charges (OPLS standard)should lead to attraction between polymer ring moieties and the solute aromatic rings.

${ }^{\mathrm{b}}$ Additionally the cut-off scheme for the treatment of molecules in adjacent simulation boxes (periodic boundaries) was changed from group to verlet. This is a technical modification to improve calculation performance as suggested in the updated user guidelines for GROMACS 5.1.2. ${ }^{[139]}$
} 
The MD run involving benzoic acid and PBA/DCM was then further analyzed by spatial distribution functions (SDFs, Figure 4-7). ${ }^{[142]}$

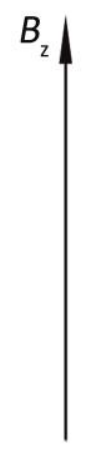

a)

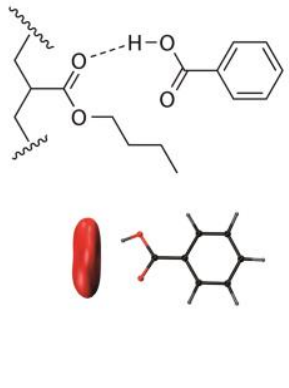

b)

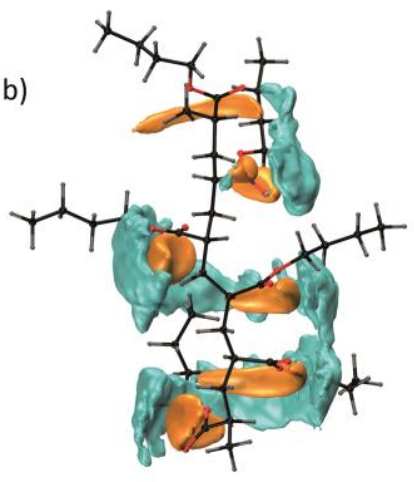

Figure 4-7: a) Top: Scheme of a possible hydrogen bond between a butyl acrylate unit of the polymer and benzoic acid. Bottom: SDF of the six ester oxygen molecules $(=\mathrm{O})$ around the benzoic acid molecule (reference frame) at an isolevel of $3 \AA^{-3}$. b) SDF of the center of mass of the benzoic acid (cyan, $2 \AA^{-3}$ ) and the six ester oxygen molecules $(=\mathrm{O})$ (orange, $7 \AA^{-3}$ ) with the simulation cell as reference frame.

These functions are a measure of the occurrence of an observed particle in a chosen reference frame. SDFs can be illustrated by 3D models which resemble the space in which the particle occurs (during the MD run) at least as often as a specified isolevel value indicates. Figure 4-7 a) bottom depicts an SDF (red) in which benzoic acid is taken as reference frame and the spatial distribution of six ester oxygen atoms $(=\mathrm{O})$ is observed. The SDF is shown at an isolevel of $3 \AA^{-3}$ which shows that during the MD run the carbonyloxygen occurs more than three times per $\AA^{3}$ within the red volume (less than three times per $\AA^{3}$ outside of the volume). In b) the simulation cell is taken as reference and the six ester oxygen atoms (=O, orange) and the center of mass of the benzoic acid (cyan) are depicted at an isolevel of $7 \AA^{-3}$ and $2 \AA^{-3}$, respectively. The SDFs in Figure 4-7 a) and b) clearly indicate the existence of hydrogen bonds by the prevalent spatial association between PBA carbonyl oxygen and benzoic acid. However, these analyses do not directly correlate hydrogen bonds with the preferred orientation of the molecules. This is achieved through so-called combined distribution functions (CDFs). ${ }^{[142]}$ In this study a CDF of a radial and an angular distribution function is derived by sampling at a time i) the distance between the carboxylate oxygen atoms of the polymer and the carboxylic hydrogen atom of the benzoic acid and ii) the associated angle between the benzoic acid C1-C4 axis and the polymer strand axis (also see Figure 44). The CDF of these two functions is given as a heat map in Figure 4-8. 


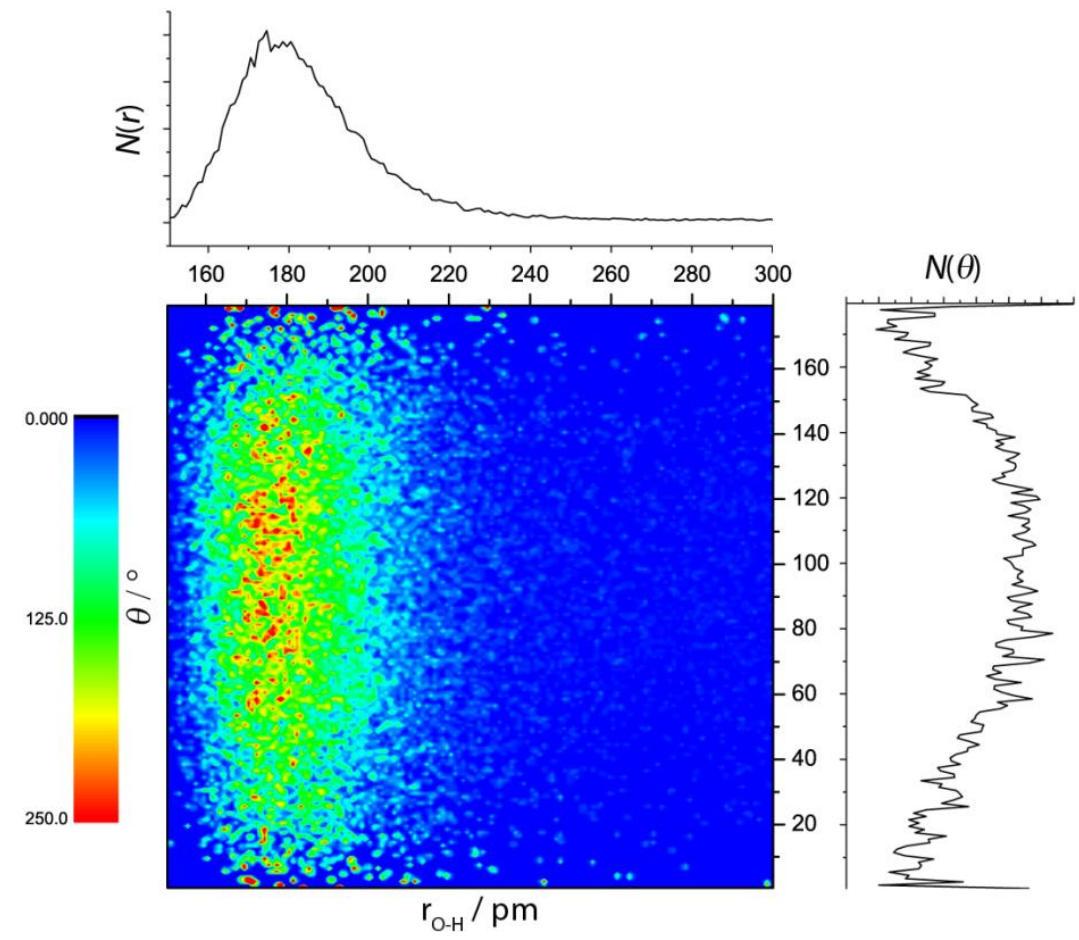

Figure 4-8: $\mathrm{CDF}$ for benzoic acid. Horizontal: radial distribution function describing the abundance of a distance between the carboxylic hydrogen of benzoic acid and the carboxylate oxygen of PBA. Vertical: angular distribution function describing the abundance of the angle between the $\mathrm{C} 1-\mathrm{C} 4$ axis (longitudinal, quasi $\mathrm{C}_{2}$ ) of benzoic acid and the polymer strand. Color-coding is according to the prevalence of the angle-distance combination.

The horizontal scale of the CDF represents the radial distribution and illustrates that short distances between 160 and $200 \mathrm{pm}$ are prevalent. These are typical values for $\mathrm{O}-\mathrm{H}$...O hydrogen bonds. ${ }^{[143]}$ These values are additionally correlated with an increased occurrence of angles around $90^{\circ}$ between polymer strand and the C1-C4 benzoic acid axis as shown by the vertical scale which represents the angular distribution. In conclusion, it is very likely, that the orientation of the molecule not only correlates with the existence of hydrogen bonds, but is caused by this directional force.

This highlights the possibility to estimate molecular alignment a priori and furthermore illustrates computational methods on how to analyse the alignment phenomena and derive the underlying mechanisms. Admittedly, the potential of MD simulations is not fully exploited in the approaches described above and experimental findings were only qualitatively reproduced. Advanced numerical simulations of alignment in nematic liquid crystals involve large ensembles of up to several thousand molecules (e.g. Pizzirusso et al. 2000 molecules with a liquid crystal molecules to solute ratio of 95:5) but which are only covered over a short period of time (e.g. Pizzirusso et al. $40 \mathrm{ns).} \mathrm{In} \mathrm{principle} \mathrm{simulation}$ of a smaller ensemble over a longer period of time is equivalent according to the ergodic hypothesis. However, it is likely that the small fixed styrene strand is only a very simplified reproduction of the real situation within the gel and certainly introduces some bias. Furthermore, improvements such as the accurate charge determination of atoms within single polymer units and incorporation of solute molecules are mandatory. In summary, these steps should lead to a drastically improved quantitative agreement between experimental and theoretical alignment. In this case, MD simulations of small 
molecules in stretched gels will add valuable information on the alignment and open up tremendous possibilities for further studies. One example is the examination of lithium organic aggregates: in standard solution NMR one cannot distinguish between various species from their chemical shift. However, aggregates align very differently and comparison of experimental and computational results can be a powerful method to evaluate the aggregation motif. Similarly, enantiomers cannot be distinguished in homogeneous solution but two enantiomers in chiral alignment media may give two discernible orientations and allow absolute structure determination through two different sets of RDCs.

\subsection{Structure Elucidation of Synthetic Indanes}

The increasing variety of small molecules, whether synthesized in the laboratory or found as natural products, requires suitable methods for their structural characterization. While the chemical constitution (i.e. the connectivity of atoms) of a molecule is often known, the determination of the configuration and conformation (i.e. the 3D structure) can become an intricate task. X-ray crystallography has the ability to doubtlessly solve these stereochemical problems but requires well grown crystals and is usually not able to make dynamical structural changes observable. ${ }^{[144]}$ Alternatively, sophisticated NMR techniques which rely on $\mathrm{NOE}^{[145]}$ as well as the analysis of $J$ couplings $^{[146]}$ are applied. However, these NMR techniques face several disadvantages since they are limited to locally well-connected spins (through bonds or spatial proximity) or depend on complex parameterized Karplus curves. ${ }^{[59,147]}$ Additionally, even if NOE and J-coupling analysis is possible, these may not resolve ambiguities that arise from unfavourable combinations of configuration and conformation as well as from fast conformational averaging. Insufficient distance and angle information from conventional NMR data can be complemented by incorporating structure verification via RDCs which involves five basic steps. ${ }^{[6,70-71,88]}$

i) Experimental determination of RDCs,

ii) Generation of a comprehensive set of possible molecular structures,

iii) Determination of alignment tensors from RDCs and structural proposals,

iv) Back-calculation of RDCs from structural proposals and alignment tensors,

v) Assessment of the agreement between back-calculated and experimental RDCs.

These steps are further explained in the following analysis of two $\beta$-amino acid esters ${ }^{[148]}$ with previously unknown configuration. $\beta$-amino acid esters are frequently employed in the synthesis of non-natural $\beta$ peptides, and are versatile intermediates in organic synthesis. ${ }^{[149]}$ Scheme 4-2 shows the reaction in which a manganese-catalyzed $\mathrm{C}-\mathrm{H}$ functionalization/alkene annulation of ketimines led to the formation of $\beta$-amino acid esters methyl-1-\{(4-methoxyphenyl)amino\}-1,5-dimethyl-2,3-dihydro- $1 H$ indene-2-carboxylate $(\beta$-aa- $\mathrm{H})$ and methyl-1-\{(4-methoxyphenyl)amino\}-1,3,5-trimethyl-2,3-dihydro$1 H$-indene-2-carboxylate $\left(\beta\right.$-aa-Me) comprising a prominent indane scaffold. ${ }^{[148]}$ 


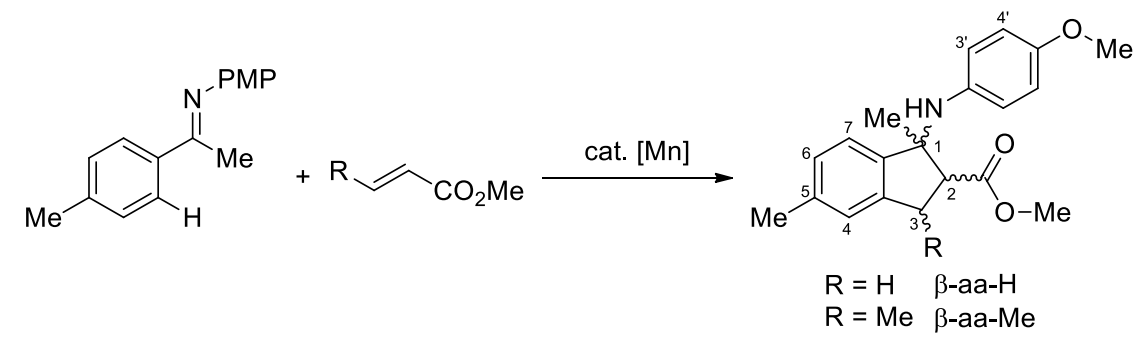

Scheme 4-2: Formation of $\beta$-aa- $\mathrm{H}$ and $\beta$-aa-Me via a manganese-catalyzed $\mathrm{C}-\mathrm{H}$ functionalization/ alkene annulation. $\mathrm{PMP}=$ para-methoxyphenyl.

Note that up to three stereogenic centres are generated in an instant out of two achiral reactants. While the constitutional elucidation of $\beta$-aa-H and $\beta$-aa-Me from common 1D and 2D NMR procedures was straightforward, the derivation of the relative configuration at $\mathrm{C} 1, \mathrm{C} 2$ and $\mathrm{C} 3$ from NOE and J-coupling data was indistinct. Both, hydrogen distances and dihedral angles, rely on the conformation of the 5membered ring which was likewise not known. For example, a pseudo-equatorial substituent on $\mathrm{C} 1$ may actually be closer in space to a trans compared to a cis configured substituent on C3 and vice versa (also see NOE and J-coupling data in the Appendix). The structure verification by RDCs is then performed by five consecutive steps that will be described in detail.

\section{i) Experimental determination of RDCs}

First, $\beta$-aa- $\mathrm{H}$ and $\beta$-aa-Me were dissolved in $\mathrm{CDCl}_{3}$ and isotropic ${ }^{1} J_{\mathrm{CH}}$ couplings were measured by CLIP-HSQC experiments. This gave $12{ }^{1} J_{\mathrm{CH}}$ couplings for $\beta$-aa-H as well as $\beta$-aa-Me. Alignment of the compounds was achieved by immersing a cylindrical PS stick (crosslinked through 0.3 vol\% divinylbenzene) in solutions of $\beta$-aa- $\mathrm{H}$ and $\beta$-aa-Me. Swelling/equilibration over four days led to sufficiently homogeneous alignment within the gel. Further CLIP-HSQC spectra were recorded to yield the couplings ${ }^{1} T_{\mathrm{CH}}$ (Figure 4-9).

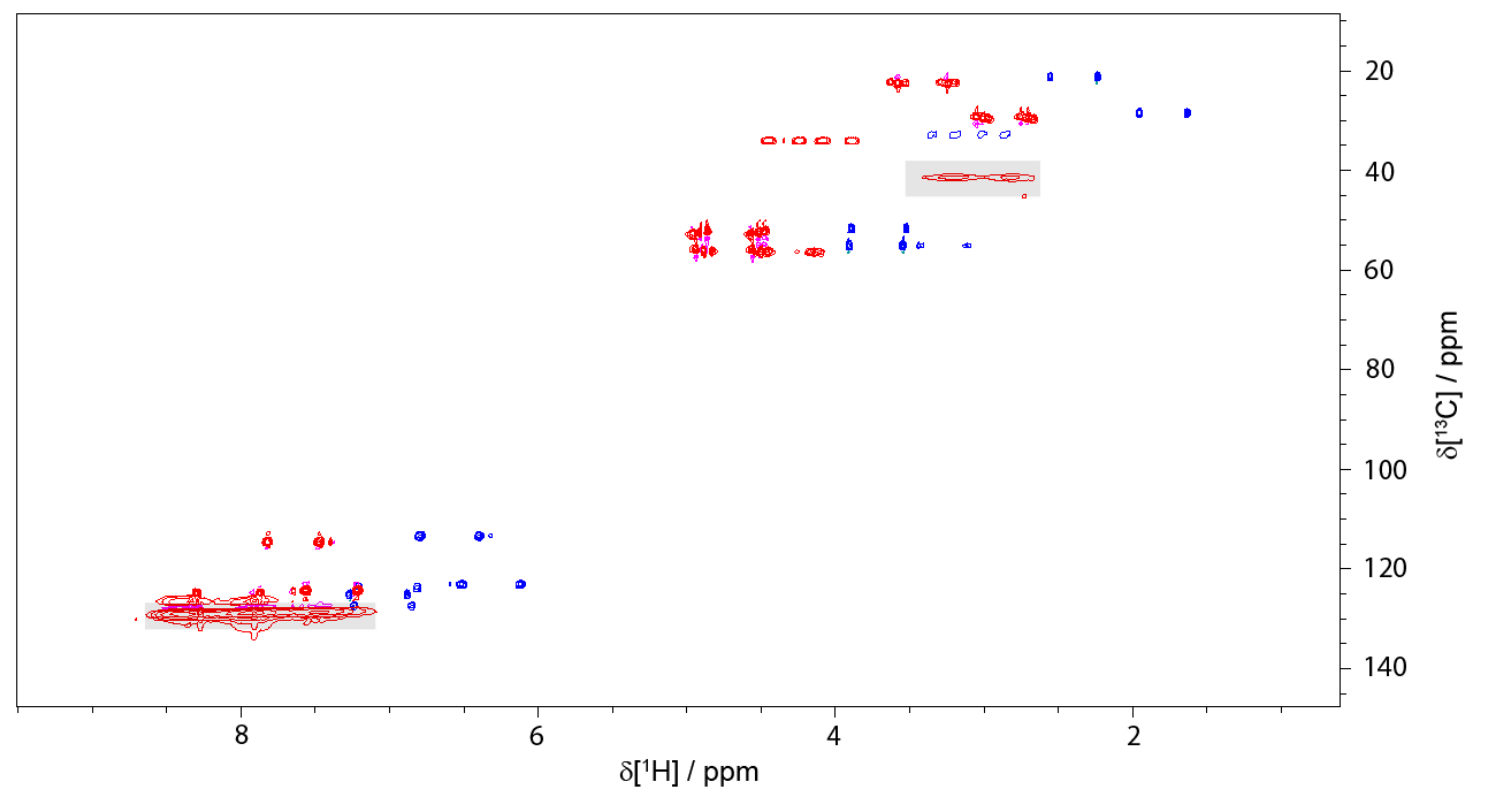

Figure 4-9: CLIP-HSQC spectra of $\beta$-aa-H. Blue: Spectrum in neat DCM (isotropic). Red: Spectrum in DCM/PBA (anisotropic, shifted in F2 dimension for clarity). Note that residual polymer signal is highlighted grey. 
The difference between isotropically and anisotropically measured couplings led to 12 RDCs (Table 4$5)$.

Table 4-5: RDCs ${ }^{1} D_{\mathrm{CH}}$ in $\mathrm{Hz}$ for $\beta$-aa-H and $\beta$-aa-Me as determined from CLIP-HSQC experiments. Numbering is according to Scheme 4-2. Experimental errors were uniformly estimated as $0.5 \mathrm{~Hz}$.

\begin{tabular}{ccc}
\hline & ${ }^{1} D_{\mathrm{CH}} / \mathrm{Hz}$ for $\beta-\mathrm{aa}-\mathrm{H}$ & ${ }^{1} D_{\mathrm{CH}} / \mathrm{Hz}$ for $\beta$-aa-Me \\
\hline $1-\mathrm{CH}_{3}$ & -6.5 & -8.9 \\
$\mathrm{C} 2-\mathrm{H} 2$ & 16.1 & 1.1 \\
$\mathrm{C} 3-\mathrm{H} 3$ & 4.5 & 13.5 \\
$\mathrm{C} 3-\mathrm{H} 3^{\circ}$ & 10.2 & \\
$3-\mathrm{CH} 3$ & & -8.8 \\
$\mathrm{C} 4-\mathrm{H} 4$ & 12.7 & -4.1 \\
$5-\mathrm{CH} 3$ & 6.6 & 10.2 \\
$\mathrm{C} 6-\mathrm{H} 6$ & -11.4 & 12.0 \\
\hline $\mathrm{C} 7-\mathrm{H}^{\mathrm{a}}$ & 11.9 & -0.5 \\
$\mathrm{C} 3-\mathrm{H}^{\mathrm{b}}$ & -18.7 & -14.5 \\
$\mathrm{C}^{\mathrm{b}}-\mathrm{H}^{\mathrm{b}}$ & -21.7 & -16.6 \\
$\mathrm{OCH}_{3}{ }^{\mathrm{b}}$ & 7.3 & 11.5 \\
$\mathrm{COOCH}_{3}{ }^{\mathrm{b}}$ & 7.3 & -2.8 \\
\hline
\end{tabular}

${ }^{a}$ Bonds C7-H7 and C4-H4 are nearly parallel and should therefore exhibit very similar RDCs. Only C4-H4 was further analyzed due to the sharper $\mathrm{H} 4$ resonance (no ortho couplings).

${ }^{\mathrm{b}}$ Non-rigid RDCs from the PMP subunit were not included in the further analysis.

Further details on sample preparation and CLIP-HSQC parameters are described in the experimental sections 5.1 and 5.2. It is most striking, that the RDCs differ quite strongly e.g. 16.1 vs. $1.1 \mathrm{~Hz}$ for C2$\mathrm{H} 2,12.7$ vs $-4.1 \mathrm{~Hz}$ for $\mathrm{C} 4-\mathrm{H} 4$ and -11.4 vs. $12.0 \mathrm{~Hz}$ for C6-H6. The sole substitution of a single hydrogen in $\beta$-aa-H by a methyl group per se should not induce this immense change in alignment that becomes obvious through these values. Instead, a more profound change, e.g. in conformation, must have occurred upon this substitution. This is additional knowledge which can be exploited as soon as the conformation of one of either structure is solved by a more detailed RDC analysis.

\section{ii) Generation of a comprehensive set of possible molecular structures}

The molecules $\beta$-aa- $\mathrm{H}$ and $\beta$-aa-Me were synthesized as racemic mixtures. The configuration of $\beta$-aa- $\mathrm{H}$ may be described as follows: $\mathrm{C} 1$ is $R$ and $\mathrm{C} 2$ is $S$ configured and the molecule is simply called $R S$. Note that in the following discussion the nomenclature of possible configurations will be analogous to this example. The enantiomer of this molecule is $S R$ (ester and amine are trans configured in both cases). Additionally, one can propose the configurations $R R$ and $S S$ (ester and amine are cis configured). Since achiral media like PS do not discriminate between enantiomers ${ }^{[150]}$ one may consider one arbitrary configuration out of these pairings (i.e. $R S$ and $S S$ ). Analogously, the possible configurations for $\beta$-aaMe are SSR (C1: S, C2: S, C3: R), SSS, RSR and RSS (implying RRS, RRR, SRS and SRR, respectively). Since the configuration of the substituents at the indane backbone is of principal interest, one may focus 
on rigid, neutral cut down structures $\beta$-aa- $\mathrm{H}^{\star}$ and $\beta$-aa-Me $\mathrm{e}^{\star}$ containing a primary amine and a carboxylic acid substituent.

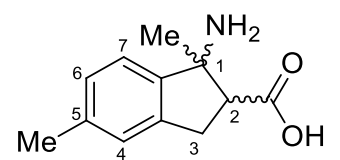

$\beta-$ aa- $H^{*}$

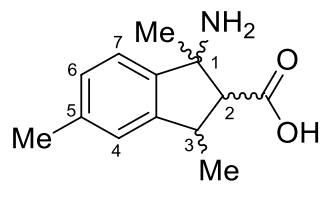

$\beta$-aa-Me*

Scheme 4-3: Cut-down structures of $\beta$-aa- $\mathrm{H}^{*}$ and $\beta$-aa-Me* .

Hence, only the RDCs in the top section of Table 4-5 were exploited. Inclusion of the PMP and ester substituents, which are linked to the indane backbone by several rotatable bonds, would add less essential information (four RDCs that further define the alignment) at the cost of a highly demanding conformational analysis. The indane scaffold of $\beta$-aa- $\mathrm{H}^{\star}$ and $\beta$-aa-Me exhibits two major conformations: the two envelope conformations in which the ester/carboxylic group and its two neighboured trans configured substituents change from pseudo-equatorial ("-eq" is added to the stereodescriptors) to pseudo-axial (“-ax”), Scheme 4-4 summarizes the process of preparing the exhaustive blueprints for possible molecular structures of $\beta$-aa-H and $\beta$-aa-Me.

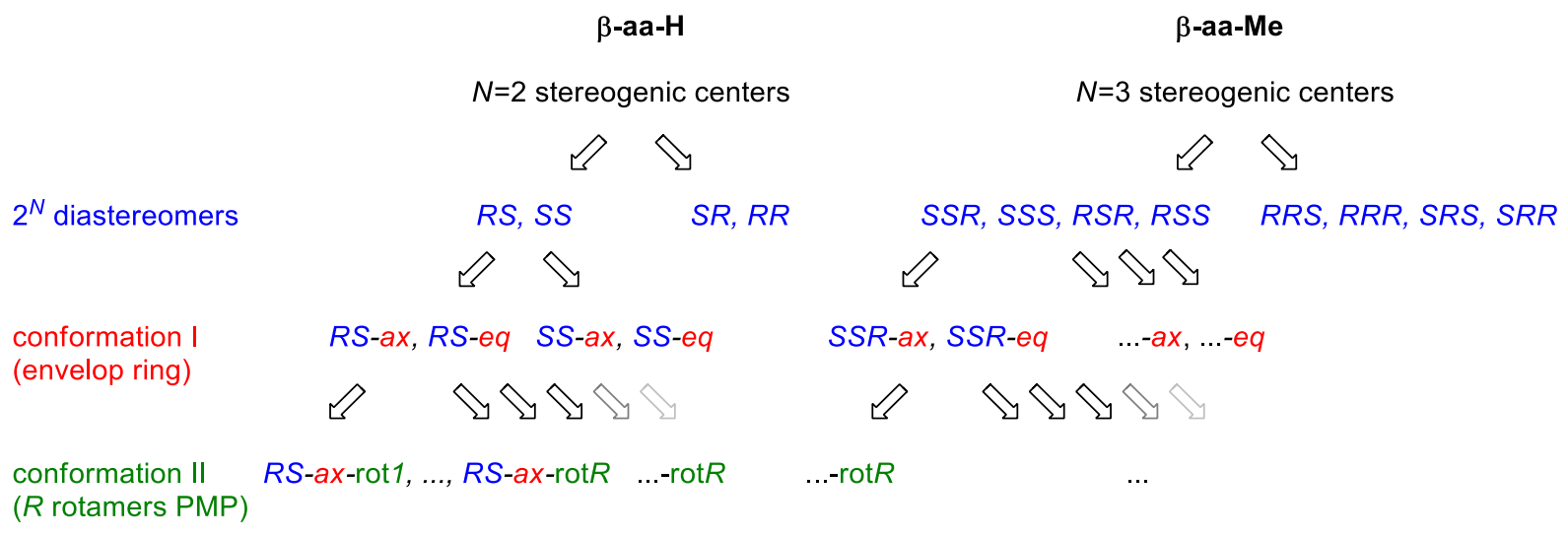

Scheme 4-4: Illustrative branching of molecules $\beta$-aa-H and $\beta$-aa-Me into possible diastereomers through different configurations (only one representative enantiomeric set can be examined since achiral PS does not allow distinction) and conformers. The latter rapidly increases the number of possible structures and should therefore be narrowed to a reasonable conformational space e.g. through cut down structures.

In general, the number of possible structures rapidly increases by a too extensive examination of the configurational and conformational space. This highlights the significance of a smart inspection and choice of structural proposals.

After generation of a comprehensive set of possible molecules with likely combinations of configurations and conformations, (non-optimized) 3D structures were created for both compounds from scratch with Chem3D Pro 14.0. This set of structures was optimized by $\mathrm{MM}^{[151]}$ as well as MMFF94 ${ }^{[152]}$ force fields. This computational level is the common demand on large biomolecules and usually yields sufficient results. ${ }^{[65 c, 65 d, 72 a, 74 a, 153]}$ As required for small molecules, the MM2 optimized set of structures was further geometry optimized on a B3LYP/def2-TZVP level of theory with ORCA 3.0.3 $3^{[154]}$. Stationary points were characterized as minima by computing numerical frequencies. Further 
details on the computational methods are described in the experimental section 5.4. The next three steps are usually wrapped up as one routine in RDC analysis software such as MSpin 2.0 Beta $^{[83]}$ which was also applied in this study.

\section{iii) Determination of alignment tensors from RDCs and structural proposals \\ iv) Back-calculation of RDCs from structural proposals and alignment tensors \\ v) Assessment of the validity of the structural proposal}

Although MSpin is stable and comprehensive with continuous technical support, it is important to understand these steps and avoid merely applying a black-box. Hence the basics of steps iii) to v) are briefly illustrated: The alignment tensor is derived in a very similar fashion to the procedure described in section 4.1. For an arbitrary molecular alignment of a $C_{1}$ symmetric molecule, linear equation systems of (1-7) and (1-4) with five unknown alignment tensor elements have to be solved. Table 4-5 indicates that these linear equation systems are once more overdetermined (seven independent RDCs, five unknown tensor elements). In the case of RDC analysis Losconzi et al. established a method called singular value decomposition ${ }^{\text {a }}$ (SVD) to obtain stable solutions for these problems. ${ }^{[155]}$

Once the alignment tensors are derived for each structural proposal, equation (1-7) can be straightforwardly applied to back-calculate RDCs for these models. The correct structural proposal will then return the best compliance between the experimental RDCs $D_{\exp }$ and back-calculated RDCs $D_{\text {calc. }}$. This is quantified for all $i$ RDCs through the Cornilsecu $Q$ factor. ${ }^{[156]}$

$$
Q=\sqrt{\frac{\sum\left(D_{\text {calc,i }}-D_{\text {exp }, \mathrm{i}}\right)^{2}}{\sum D_{\text {exp }, \mathrm{i}}}}
$$

Hence, the smallest $Q$ factor indicates the most suitable structural proposal. These steps were first performed via MSpin for $\beta$-aa- $\mathrm{H}^{\star}$. Note that for $\beta$-aa- $\mathrm{H}^{\star}$ it is not possible to a priori assign the prochiral hydrogen atoms at C3. Instead, a full analysis was performed in which the involved couplings are interchanged. An additional suffix " $(i)$ " signals that the coupling C3-H3 $(4.5 \mathrm{~Hz})$ is assigned to the pro- $S$ hydrogen and $\mathrm{C} 3-\mathrm{H} 3^{\circ}(10.2 \mathrm{~Hz})$ to the pro- $R$ hydrogen (for "(ii)" vice versa). This results in eight structural proposals from a permutation of two configurations, two conformations and two possible assignments for $\beta$-aa- $\mathrm{H}^{\star}$ (for a detailed exemplary MSpin input see the Appendix). Figure 4-10 shows the resulting $Q$ factors for this set.

\footnotetext{
${ }^{\text {a }}$ Iterative least-square methods as applied in section 4.1 are more suitable if previous knowledge of the alignment tensor is available. This is usually the case for symmetric molecules.
} 


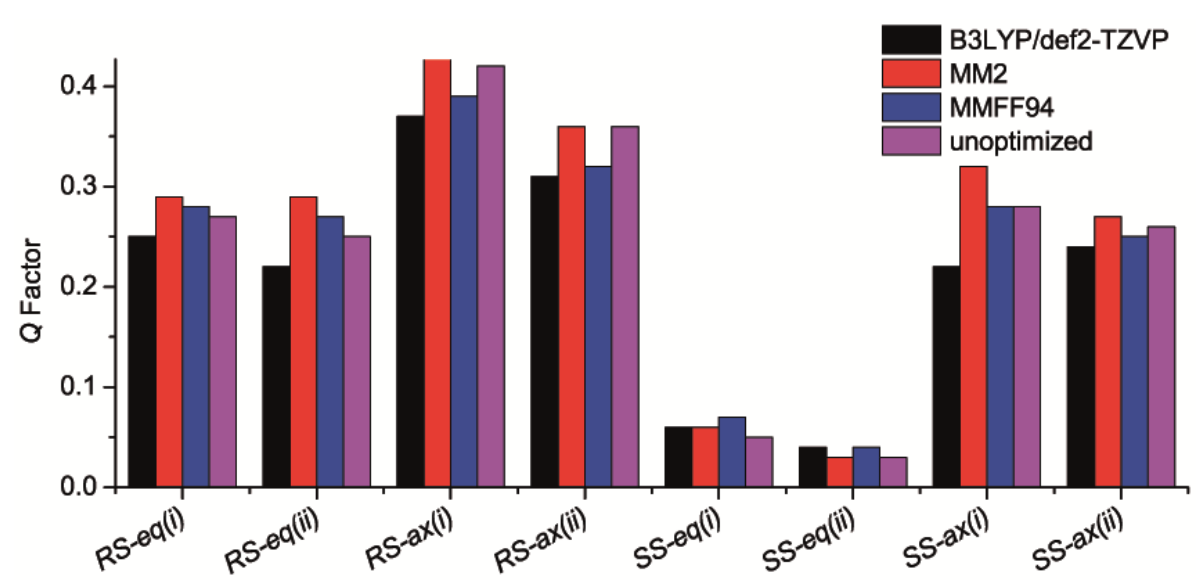

Figure 4-10: $Q$ factors calculated using seven RDCs (Table 4-5) for the set of configurations/conformations/assignments of $\beta$-aa- $\mathrm{H}^{*}$. Each structure was optimized by different theoretical methods (B3LYP/ def2-TZVP, MM2, MMFF94, unoptimized textbook angles). The suffix "(i)" signals that the coupling $\mathrm{C} 3-\mathrm{H} 3(4.5 \mathrm{~Hz})$ is assigned to the pro-S hydrogen and $\mathrm{C} 3-\mathrm{H} 3^{\circ}(10.2 \mathrm{~Hz})$ to the pro- $R$ hydrogen (for "(ii)" vice versa).

According to Figure 4-10, $Q$ factors for SS-eq(i) and SS-eq(ii) ( 0.05) are more than four times smaller than corresponding values for other feasible structures $(\sim 0.22)$. Therefore, the substituents in $\beta$-aa- $\mathrm{H}^{*}$ are cis configured. This is in accordance with the proposed reaction mechanism which suggests chelation of the manganese catalyst by the ester and amine moieties. ${ }^{[148]}$

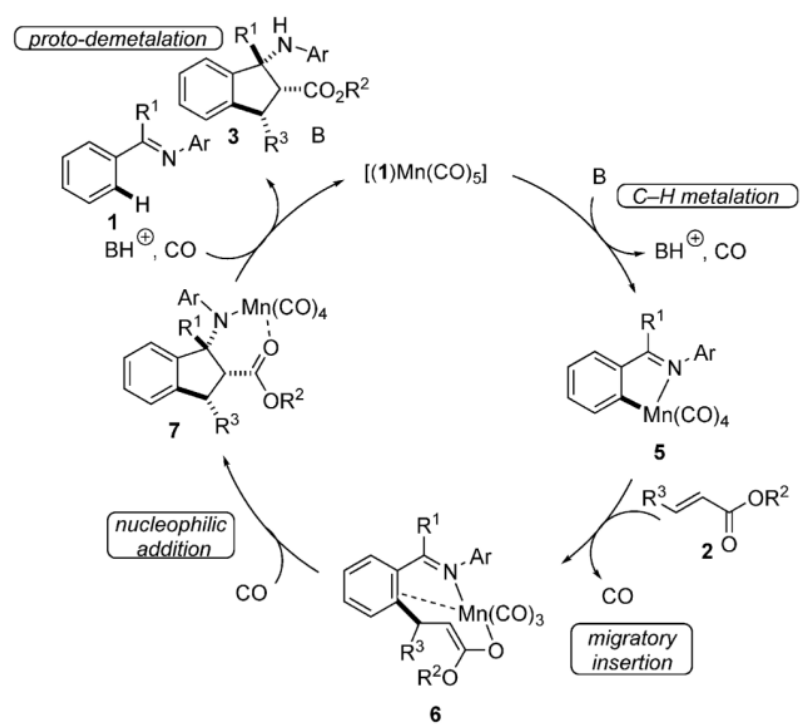

Scheme 4-5: Proposed mechanism for the manganese-catalyzed synthesis of cis- $\beta$-amino acid esters through organometallic $\mathrm{C}-\mathrm{H}$ activation of ketimines. Reprint with permission from reference [148]. Copyright 2016 Wiley $\mathrm{VCH}$.

Although the barrier for a ring flip is usually low for indanes, the pseudo-equatorial orientation of the ester within the five-membered ring is preferred. ${ }^{[157]}$ A more advanced fitting of an equilibrium between $R S-e q / R S$ - $a x$ and SS-eq/SS-ax (DFT structures) with a single tensor approach, ${ }^{a}$ as implemented in

a Since alignment tensors for two different conformers are usually different from each other, the analytic solution depends on 1 population parameter and $2 \times 5$ alignment tensor elements. The number of RDCs available is less 
MSpin, only slightly improved the $Q$ factor at population ratios clearly favouring the equatorial conformer.

Table 4-6: Population fitting of two possible conformers for each diastereomer of $\beta$-aa- $\mathrm{H}^{\star}$ (DFT structures). The single tensor approach as implemented in MSpin 2.0 Beta was applied for this procedure. Note that the reported values for the single conformers result from the adapted alignment tensor and thereby differ from the values reported above.

\begin{tabular}{lllll}
\hline & $\begin{array}{l}\text { Eq-conformer } \\
\text { Q Factor }\end{array}$ & $\begin{array}{l}A x \text {-conformer } \\
Q \text { Factor }\end{array}$ & $\begin{array}{l}\text { Population } \\
(- \text { eq }:-a x)\end{array}$ & $\begin{array}{l}\text { Equilibrium } \\
\text { Q Factor }\end{array}$ \\
\hline$R S($ i $)$ & 0.22 & 0.88 & $1: 0$ & 0.22 \\
$R S($ ii $)$ & 0.22 & 0.74 & $1: 0$ & 0.22 \\
$S S($ i $)$ & 0.05 & 0.71 & $1: 0$ & 0.05 \\
$S S($ ii $)$ & 0.05 & 0.54 & $0.94: 0.06$ & 0.04 \\
\hline
\end{tabular}

It is striking that DFT calculations for the SS configuration gave only a minor energy difference of $-0.8 \mathrm{~kJ} / \mathrm{mol}$ favouring the pseudo-equatorial over the axial conformation. This discrepancy may stem from the simplified single tensor approach which underestimates the axial population. In general, the treatment of highly flexible molecules requires more delicate approaches, yet the indanes are rather rigid. ${ }^{[158]}$ Hence, it seems more likely that approximations which enable reasonably expensive DFT calculations led to this discrepancy between experiment and theoretical prediction. For instance, the incorporation of the oPMP unit in DFT calculations will on one hand change the energy of the system through intramolecular interactions. On the other hand, the oPMP unit as well as the aromatic indane scaffold may both (very weakly) interact with the PS strands. This effect is strongly suggested by the results for attractive interaromatic forces between small aromatic molecules and PS as shown in section 4.1. In the case of $\beta$-aa- $\mathrm{H}$, a combination of interaromatic forces and generalized repulsive/steric forces certainly adds energy terms which change (supposedly increase) the gap between the pseudo-equatorial and the axial conformation. This has a delicate consequence on structure elucidation via RDCs: Interaction between alignment medium and aligned molecule may, contrary to results from former studies, ${ }^{[159]}$ bias the conformation of the observed molecule (configurations are still doubtlessly derived). The conformation of a molecule plays an important role for its reactivity, stereoselectivity etc. Hence, wrong assumptions from biased experimental data must be avoided by supportive high-level theoretical methods.

Although modern RDC studies on small molecules usually employ at least DFT level structure optimization, remarkably accurate results can also be obtained at very low computational cost. As shown by Figure 4-10 MD simulations and most basic textbook distances and angles (unoptimized) can be employed to obtain the correct result. Even the minute distinctions between SS-eq(i) and SS-eq(ii)

than this. Therefore, approximations are made in which atoms of two conformations are superimposed according to least-squares methods. This yields a shared coordinate system with a single alignment tensor. An equation in the form of population weighted sums of (1-7) is then solved. Detailed procedures are described in the MSpin reference manual. 
are likewise reproduced by all four methods and allow a conclusive assignment of the prochiral hydrogen atoms at $\mathrm{C} 3$ in $\beta$-aa- $\mathrm{H}^{*}$. Although only marginally better (smaller) $Q$ values were found for DFT structures of SS-eq(ii) over SS-eq(i), it is safe to assume that SS-eq(ii) is in fact the true assignment. With the knowledge that ester and amine groups are cis configured and the ester is predominantly in a pseudo-equatorial position the correct assignment SS-eq(ii) can be confirmed by the NOE contacts. E.g. the integral for the cross-peak between H4 (aromatic ring plane) and pseudo-axial H3 ${ }^{\text {pro- } R}(-0.44)$ is much smaller than the integral for the cross-peak between $\mathrm{H} 4$ and pseudo-equatorial $\mathrm{H} 3^{\text {pro-s }}(-0.75)$ indicating closer contacts for the latter pair of protons. Further experimental information and data on the supplementary NOESY experiment is given in the Appendix B (Extended NMR Spectroscopy of Synthetic Indanes).

Successful structure verification of $\beta$-aa- $\mathrm{H}^{\star}$ facilitates the subsequent analysis of $\beta$-aa-Me*. Since both compounds result from analogous syntheses, the cis configuration between ester and amine substituents will not be altered. ${ }^{a}$ Consequently, four initially possible configurations are reduced to two stereoisomers that differ in the relative configuration of the methyl group at C3: SSR and SSS (see Figure $4-11$ ). Yet, the full set of permutations was examined via MSpin ( 4 configurations $\times 2$ conformations for $\beta$-aa-Me ${ }^{\star}$.

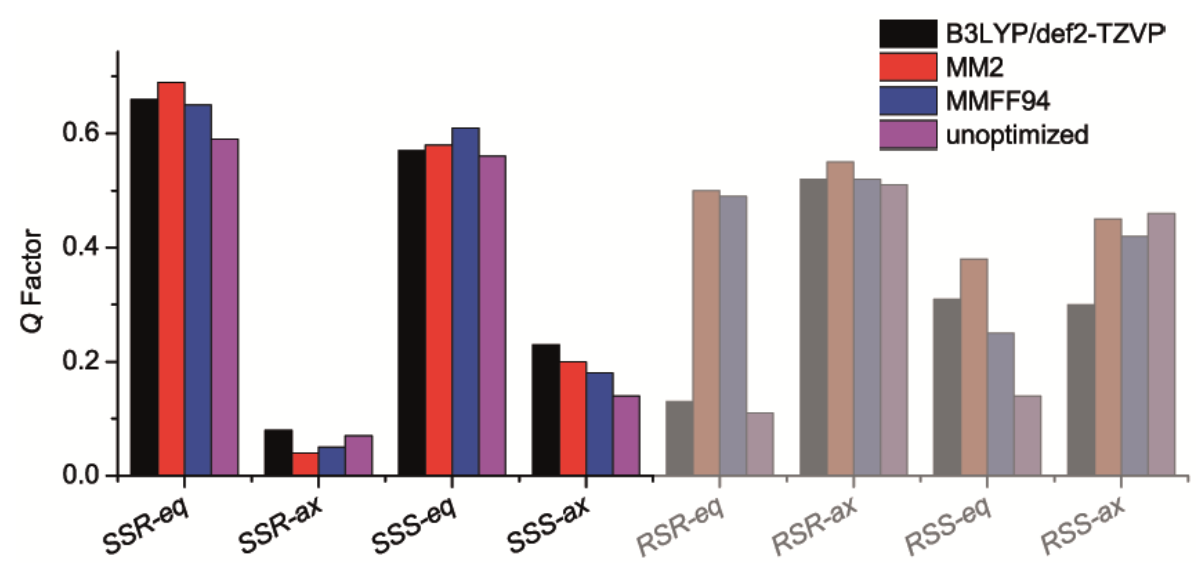

Figure 4-11: $Q$ factors calculated using seven RDCs (Table 4-5) for the set of configurations/conformations of $\beta$ aa-Me* ${ }^{\star}$, each optimized by different theoretical methods. Four structures are greyed out which differ in the substituent at $\mathrm{C} 1$. These can be ignored due to results for the structurally analogous compound $\beta$-aa- $\mathrm{H}^{*}$.

Here, SSR-ax provides the smallest $Q$ values regardless of the optimization method. Population fitting (DFT structures) gave some remarkable results for the full set.

${ }^{\text {a }}$ Chelating of the manganese catalyst by the amine and carboxylic function as shown in Scheme 4-2 will naturally lead to cis configured ester and amine substituents. 
Table 4-7: Population fitting of two possible conformers for each diastereomer of $\beta$-aa-Me* (DFT structures). The single tensor approach as implemented in MSpin 2.0 Beta was applied for this procedure. Note that the reported values for the single conformers result from the adapted alignment tensor and thereby differ from the values reported above.

\begin{tabular}{lllll}
\hline & $\begin{array}{l}\text { Eq-conformer } \\
Q \text { Factor }\end{array}$ & $\begin{array}{l}\text { Ax-conformer } \\
\text { Q Factor }\end{array}$ & $\begin{array}{l}\text { Population } \\
(-e q:-a x)\end{array}$ & $\begin{array}{l}\text { Equilibrium } \\
\text { Q Factor }\end{array}$ \\
\hline$S S R$ & 1.09 & 0.07 & $0: 1$ & 0.07 \\
$S S S$ & 1.02 & 0.23 & $0: 1$ & 0.23 \\
$R S R^{\mathrm{a}}$ & 0.33 & 2.65 & $0.89: 0.11$ & 0.10 \\
$R S S^{\mathrm{a}}$ & 0.83 & 1.27 & $0.60: 0.40$ & 0.07
\end{tabular}

a Structures RSR and RSS can be omitted due to the results from $\beta$-aa-H.

The possible configurations SSR and SSS showed only pseudo-axial conformers and therefore no improvement of the $Q$ factors. RSR and RSS can be excluded as possible configurations but exhibit remarkably good $Q$ values. If no preceding analysis of $\beta$-aa- $\mathrm{H}$ had been made, these structures would impose some additional efforts on the structure validation of $\beta$-aa-Me.

The single conformer solution SSR-ax with no pseudo-equatorial contribution is also supported by DFT calculations which gave an energy difference of $-9.3 \mathrm{~kJ} / \mathrm{mol}$ in favour of the pseudo-axial over the equatorial conformer. Compared to the next best solution which is SSS-ax, Q factors found for SSR-ax are smaller by a factor of more than two. A direct comparison between measured and back-calculated RDCs for SSR-ax and SSS-ax is shown in Figure 4-12.

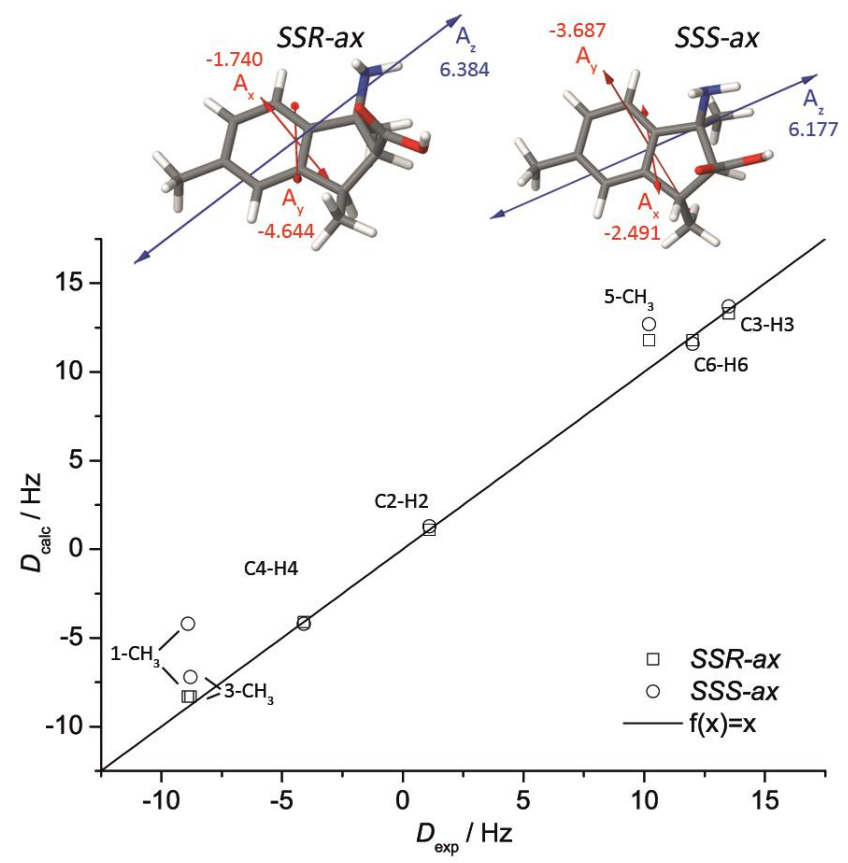

Figure 4-12: Top: B3LYP/def2-TZVP structures for SSR-ax and SSS-ax with vectors representing the principal axes of the alignment tensor (alignment tensor elements are given in units of $\left.10^{-4}\right)$. Bottom: Back-calculated $\left(D_{\text {calc }}\right)$ vs. measured $\left(D_{\text {exp }}\right)$ RDCs for SSR-ax and SSS-ax. Notably 1- $\mathrm{CH}_{3}$ and $3-\mathrm{CH}_{3}$ exhibit stronger deviations between experimental and back-calculated RDCs. 
Due to similar alignment tensors, nearly identical back-calculated RDCs result for C2-H2, C3-H3, C4H4, C6-H6 and C7-H7. However, both isomers are clearly distinguished by the back-calculated RDCs for $1-\mathrm{CH}_{3}, 3-\mathrm{CH}_{3}$ and $5-\mathrm{CH}_{3}$ which in SSR-ax agree much better with the experimental RDCs. These RDCs in particular affect the bonds (configurations) that are under discussion and hence, leave no doubt about the correct structure being SSR-ax.

The axial preference of the ester substituent in $\beta$-aa-Me (as opposed to an equatorial preference in $\beta$-aa$\mathrm{H}$ ) is highly remarkable. This is most likely due to steric repulsion between the large amine substituent at C1 and the methyl group at C3 (both pseudo-axial) in SSR-eq. This was already anticipated by the large deviations between the RDCs of both compounds (see Table 4-5). In summary, from the lowest absolute $Q$ factors and the best agreement between measured and back-calculated RDCs for the crucial bonds it is possible to select SSR-ax as the likely correct structure of $\beta$-aa-Me. However, this conclusion is strongly facilitated by the previous analysis of $\beta$-aa- $\mathrm{H}$ which excluded the configurations $R S R$ and $S S R$. In fact, DFT structures of SSR-ax and RSR-eq give very similar $Q$ factors. Hence, a fast and reliable method to further exclude $R S R$-eq is desirable. Fortunately, results on different geometry optimization methods for RSR-eq already conveyed a strong dependence of the $Q$ factor on (minor) structural changes. This led to the implementation of a further criterion on structure validity: $Q$ factor stability against addition of structural noise.

\subsubsection{Structural Error Analysis}

MSpin allows an estimate of confidence for $Q$ factors through RDC errors (uniformly set to $0.5 \mathrm{~Hz}$ for the study above as indicated in Table 4-5) that are statistically analyzed. ${ }^{[83]}$ Furthermore, within MSpin the stability (validity) of the SVD technique is indicated by so-called condition numbers $(<30$ is suggested to be reliable). Herein, SSR-ax and RSR-eq give similar standard deviations for $Q$ factors (0.012 and 0.013, respectively) and inconspicuous SVD condition numbers (8.9 and 17.4, respectively). From these measures, the DFT-optimized structure RSR-eq is a valid structural proposal. However, results for different optimization methods of RSR-eq have shown that the low $Q$ factor is not stable, but can rapidly increase upon minor structural changes.

In order to obtain an estimate of the reliability of the $Q$ factors, structural noise was added to four B3LYP/def2-TZVP optimized structures that cover a range of $Q$ factors: SSR-ax with $Q=0.08$ (most probable), $R S R-e q$ with $\mathrm{Q}=0.13$ (highly probable), $S S S$ - $a x$ with $Q=0.23$ (fairly probable) and SSR-eq with $Q=0.66$ (highly improbable). All these structures possess SVD conditions number $<30$ which is suggested to be reliable. By using a simple Python script an ensemble of 1000 molecular models was created for each structure in which the atomic coordinates of the substituents on C1, C2 and C3 were randomly varied within individual spheres of a maximum radius $\Delta r_{\max }$ of $0.01 \AA$ (the corresponding script can be found in the Appendix B). 


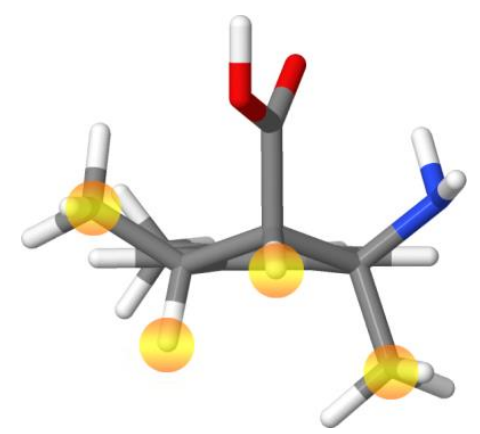

Figure 4-13: Structure SSR-ax optimized by MM2 force fields with illustrations of radially exaggerated spheres in which the corresponding substituent is randomly displaced.

Subsequently, this procedure was repeated for all structures with maximum random displacements of $0.02,0.03$ and $0.04 \AA$ A. Finally, $Q$ values were determined for all these ensemble structures via MSpin. In order to quantify the stability of the $Q$ factors, standard deviations $\sigma$ were determined. The results of this procedure are summarized in Figure 4-14 a).
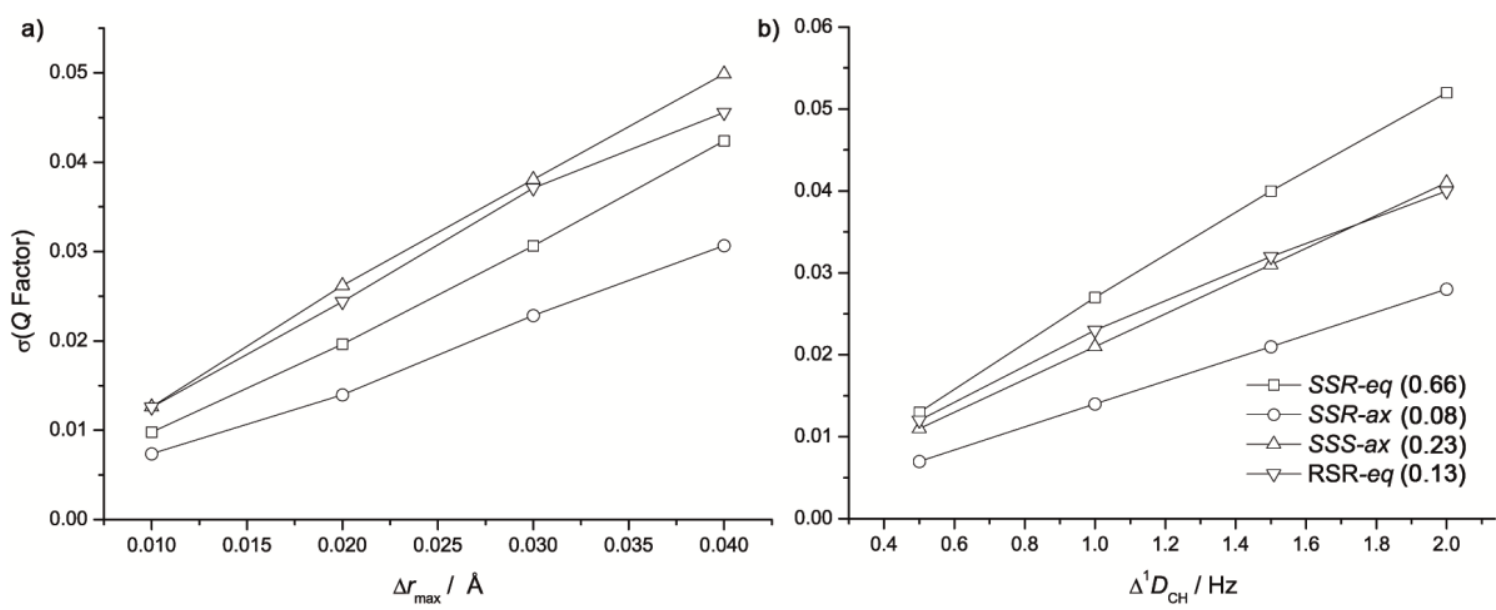

Figure 4-14: a) Standard deviations $\sigma$ of the $Q$ factors for the four exemplary structures SSR-ax, RSR-eq, SSS- $a x$ and SSR-eq against the maximum random displacement $\Delta r_{\max }$ of the coordinates of the substituents on $\mathrm{C} 1, \mathrm{C} 2$, and C3. b) Standard deviation of the $Q$ factors $\sigma$ for the four exemplary structures against the maximum statistical error for the RDCs $\Delta^{1} D_{\mathrm{CH}}$ of the internuclear vectors attached to $\mathrm{C} 1, \mathrm{C} 2$, and $\mathrm{C} 3$.

Figure 4-14 a) shows that structure SSR-ax with the lowest $Q$ factor is also least vulnerable against random displacement of significant atoms. Even though $Q$ factors for the other structures are quite diverse, their standard deviations are agglomerated and about twice as high as for SSR-ax. The only other plausible structure which is SSS-ax (considering the results for $\beta$-aa-H and the analogous synthesis of $\beta$-aa-Me), actually showed the highest standard deviation. Hence, $Q$ factor stability against addition of structural noise is indeed a valuable criterion in structure verification/falsification.

Note that besides this structural error analysis, an elaborate RDC error analysis may yield similar results. To evaluate this complementary approach the experimental errors of the RDC for $\beta$-aa-Me* $\Delta^{1} D_{\mathrm{CH}}$ was set to zero, except for the RDCs associated to C1, C2, C3 and their substituents which were set to $0.5 \mathrm{~Hz}$. Next, the structures were analysed again with MSpin and standard deviations $\sigma$ were directly obtained as MSpin output. This procedure was repeated for $\Delta^{1} D_{\mathrm{CH}} 1.0,1.5$ and $2.0 \mathrm{~Hz}$. The results of this procedure are summarized in Figure 4-14 b). Obviously, this experimental error analysis 
is also a sophisticated criterion in structure verification/falsification, as again the correct structure SSR$a x$ yields the least deviations compared to the other structures. However, the order of validity for a possible configuration differs between structural und experimental noises (validity structural noise: $S S R-a x>S S R-e q>R S R-e q \approx S S S-a x$, experimental noise: SSR-ax $>R S R-e q \approx S S S-a x>S S R-e q)$. Therefore, both methods may be used complementary.

MSpin directly yields the standard deviation of $Q$ factors for the experimental noise without further possibilities to analyse the resulting $Q$ factor distributions. In contrast, the results obtained from random noise can be further examined by binning the values for the 1000 generated structures within steps of 0.005. Results are depicted in Figure 4-15.
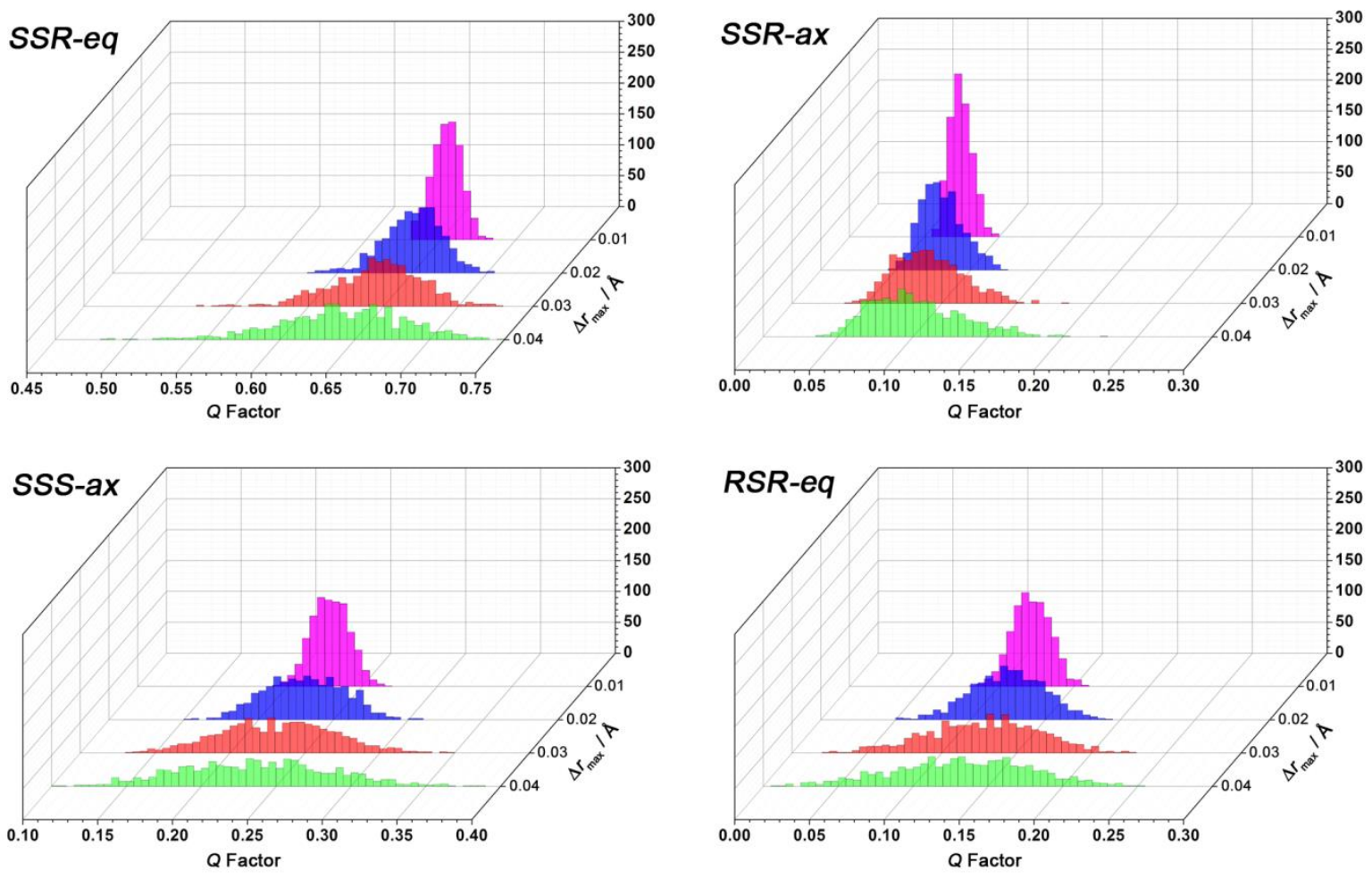

Figure 4-15: Distributions of $Q$ factors for the four exemplary structures SSR-ax, RSR-eq, SSS-ax and SSR-eq resulting from the maximum random displacement $\Delta r_{\max }$ of the coordinates of the substituents on $\mathrm{C} 1, \mathrm{C} 2$, and C3. Values are binned within steps of $\Delta Q=0.005$.

The shapes of these distributions are most noticeable: the three incorrect structures exhibit typical Gaussian-like symmetric distributions centred at the $Q$ value of the parent structure with equally broad tails towards low and high $Q$. For instance, $R S R$-eq gives astonishingly small $Q$ factors. In contrast, the distribution for SSR-ax is generally sharper and and has a higher skewness, in particular, it exhibits a steep decline towards the low $Q$ value cut-off close to the parent $Q$. Hence, the optimized structure for SSR-ax represents the ensemble minimum and the best possible agreement with the experimental RDCs. These observations allow a qualitative discrimination. A simple quantitative discrimination is 
achieved by fitting Gaussian probability functions and inspection of the goodness of these fits e.g. through the reduced chi-squared $\chi_{\mathrm{v}}{ }^{2}$ (Figure $\left.4-16\right){ }^{\mathrm{a}}$
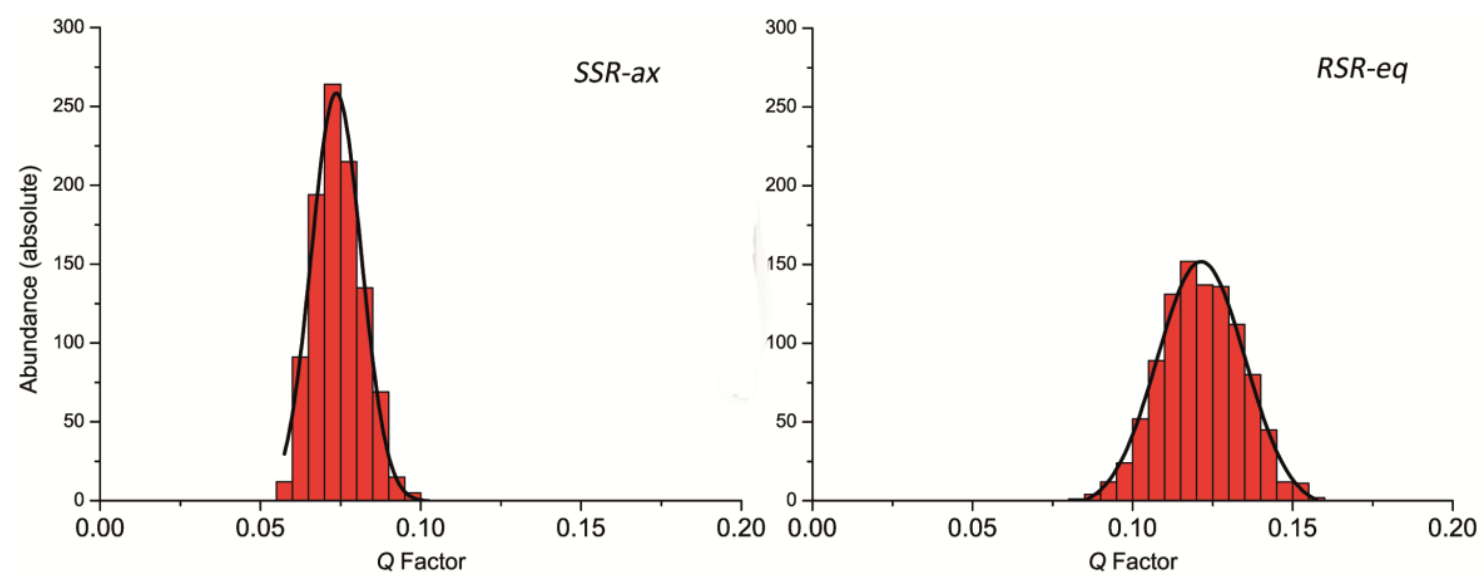

Figure 4-16: Distribution of $Q$ factors and Gaussian fit for SSR-ax and RSR-eq, SSR-ax $\left(Q=0.08, \chi_{\mathrm{v}}{ }^{2}=156.8\right)$ and $R S R-e q\left(Q=0.13, \chi_{\mathrm{v}}^{2}=31.8\right)$ at a maximum random displacement $\Delta r_{\max }=0.01 \AA$.

A summary for $Q$ factors, standard deviations $\sigma\left(\Delta r_{\max }=0.01 \AA\right)$ and reduced chi-squared $\chi_{\mathrm{v}}^{2}$ $\left(\Delta r_{\max }=0.01 \AA\right)$ is given in Table $4-8$.

Table 4-8: $Q$ values, standard deviations $\sigma(Q)$ for $\Delta R_{\max }=0.01 \AA$ and reduced chi-squared $\chi_{\mathrm{v}}^{2}$ for $\Delta R_{\max }=0.01 \AA$ for structures SSR-eq, SSR-ax, SSS-ax and RSR-eq. The latter criterion shows the most distinguished differences between wrong and true structures.

\begin{tabular}{ccccc}
\hline & SSR-eq & SSR-ax & SSS-ax & RSR-eq \\
\hline $\begin{array}{c}Q \\
(\text { MSpin })\end{array}$ & 0.66 & 0.08 & 0.23 & 0.13 \\
\hline $\begin{array}{c}\sigma(Q) \\
\Delta r_{\max }=0.01 \AA\end{array}$ & 0.020 & 0.014 & 0.026 & 0.024 \\
\hline $\begin{array}{c}\chi_{\mathrm{v}}^{2} \text { (Gauss) } \\
\Delta r_{\max }=0.01 \AA\end{array}$ & 37.8 & 156.8 & 21.9 & 31.8 \\
\hline
\end{tabular}

This additional criterion obviously enables a very pronounced discrimination compared to $Q$ factors alone. The peculiar features of $Q$ factor distributions upon addition of structural noise may be explained i) by a structure that gives a large or accidentally low $Q$ factor both of which behave stochastically and give Gaussian (normal) distributions and ii) by the correct structure with a good $Q$ factor that remains largely unchanged upon addition of noise (structural uncertainty is inherent in experimental RDCs due to vibrations etc.) or can only be biased to higher $Q$ factors (resembles a $Q$ factor minimum).

Thus, RDC structure elucidation may significantly benefit from this criterion as it may resemble an absolute criterion, i.e. no full set of structures has to be examined and compared, but as soon as the nonGaussian solution is found the corresponding structure is verified. Further (empirical) studies are

\footnotetext{
${ }^{a}$ A range of specifically designed methods exists to prove normal distributions of populations and base statistically valid descriptions on this (e.g. Shapiro-Wilk test ${ }^{[160]}$ ). The application of these methods was beyond the scope of this work, instead the bell-curve fitting and goodness-of-fit approach was which is largely determined by the skewness of the distribution.
} 
needed to put this hypothesis to the test and (possibly) show its limits according to the number of RDCs.

\subsection{Quantitative Analysis of Residual Quadrupolar Couplings}

Lithium organic compounds show interesting variations in coordination number, ligand identity and molecular symmetry as shown in section 1.1. The clarification of these structural features in solution enables the derivation of structure reactivity relationships which in turn allow the smart design of lithiation reactions. DOSY molecular weight determination is a useful method to distinguish between aggregates of different sizes/MWs (e.g. monomers, dimers, trimers or coordination by one, two, three solvent molecules). ${ }^{[33-34,36]}$ Nevertheless, hypothetical aggregates may exhibit very similar MWs (Scheme 4-6) which cannot be unambiguously assigned to experimental findings from DOSY.

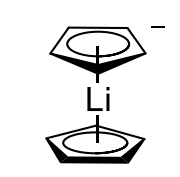

$137 \mathrm{~g} / \mathrm{mol}$

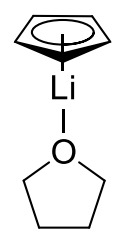

$144 \mathrm{~g} / \mathrm{mol}$

Scheme 4-6: Two hypothetical aggregates of CpLi in THF. Both molecules show molecular weights around $140 \mathrm{~g} / \mathrm{mol}$. These minute differences are not resolvable with the established MW determination by DOSY.

Traditionally, solid state NMR spectroscopy has been invested in the examination of quadrupolar couplings to obtain information about the EFG and thereby clarify structures (also see subsection 1.3.2) ${ }^{[61 a, 161]}$ For example, the aggregates in Scheme $4-6$ will exhibit considerably different EFGs around the lithium nuclei (highly symmetric, $D_{5 d}$ sandwich complexes exhibit only minute charge imbalances around the central nucleus i.e. small EFGs ${ }^{[162]}$ ). In solid state NMR, $\chi$ and $\eta$ are obtained with ease from lineshape-fitting of the quadrupolar coupling as given by equation (1-11). ${ }^{[85-86]}$ Main group elements ${ }^{[163]}$ but also heavy transition metals ${ }^{[164]}$ represent popular objectives for these heteronuclear NMR experiments. Yet, in isotropic solution this valuable structural information gets lost.

Fortunately, NMR spectroscopy in uniaxial media enables an elegant recovery of quadrupolar couplings. These RQCs are straightforwardly correlated to the EFG $\left(V_{\mathrm{ij}}\right)$ by formula (1-13).

$$
v_{\mathrm{rq}}=\frac{3 e Q}{2 I(2 I-1) h} \sum_{\mathrm{i}, \mathrm{j}=\mathrm{x}, \mathrm{y}, \mathrm{z}} V_{\mathrm{ij}} A_{\mathrm{ij}}
$$

Additionally, first principles prediction of EFG parameters by theoretical means can nowadays be achieved with great success. ${ }^{[162 b, 163 a, 163 e, 163 \mathrm{f}, 163 \mathrm{~h}, 164 \mathrm{~d}]}$ Hence, within this work a new method for structure determination of lithium organic aggregates is outlined that involves six basic steps:

i) Experimental determination of RDCs and RQCs

ii) Generation of a comprehensive set of possible molecular structures

iii) Determination of alignment tensors from RDCs and structural proposals 
iv) Determination of EFGs from complementary techniques

v) Calculation of theoretical RQCs from alignment tensor and EFG

vi) Assessment of the agreement between calculated and experimental RQCs

These steps are further explained in the following case study of lithium cyclopentadienide in THF. ${ }^{[17 d, 165]}$

\section{Experimental determination of RDCs and RQCs}

First, CpLi was dissolved in THF and a single isotropic ${ }^{1} J_{\mathrm{CH}}$ coupling was measured by CLIP-HSQC. Alignment of the compound was achieved by immersing a cylindrical PS stick (crosslinked through 0.4 vol\% divinylbenzene) in a solution of CpLi in THF. Swelling/equilibration over eight days led to sufficiently homogeneous alignment within the gels. The magnitude of the RQC $\left|v_{\text {rqeexp }}\right|$ was determined from the chemical shift differences between the central transition and the satellite peaks from a ${ }^{7} \mathrm{Li}$ spectrum (Figure 4-17).

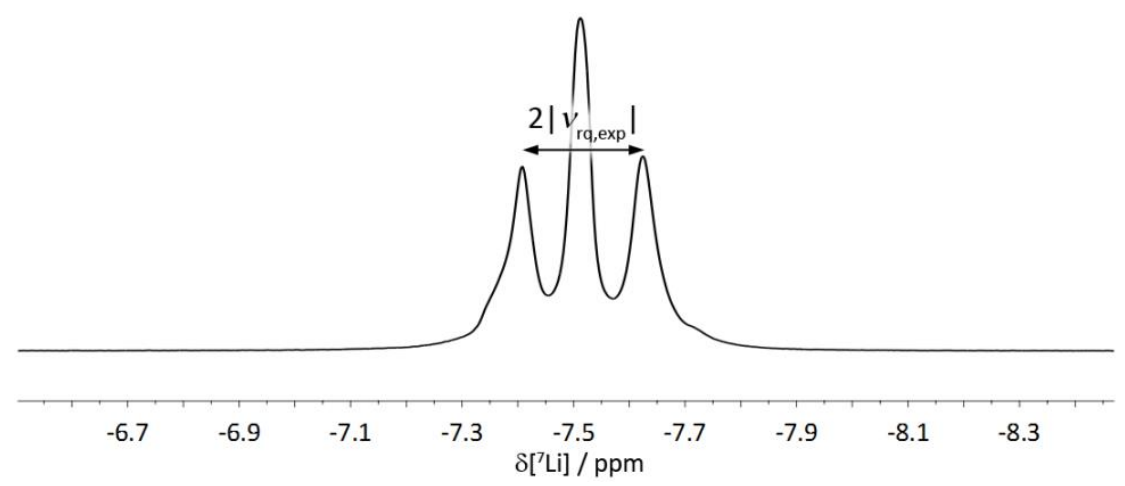

Figure 4-17: ${ }^{7} \mathrm{Li}$ spectrum of CpLi in THF/PS (0.4\% DVB) eight days after sample preparation.

A CLIP-HSQC spectrum was recorded to yield the coupling ${ }^{1} T_{\mathrm{CH}}$. The difference between isotropically and anisotropically measured couplings led to the RDC listed in Table 4-5.

Table 4-9: $\mathrm{RDC}{ }^{1} D_{\mathrm{CH}}$ in $\mathrm{Hz}$ for $\mathrm{CpLi}$ in THF/PS (4\% DVB) as determined from CLIP-HSQC experiments. Experimental errors were determined as standard deviations in repeating manual peak picking attempts.

\begin{tabular}{cc}
\hline$\left|v_{\text {rqeexp }}\right| / \mathrm{Hz}$ & ${ }^{1} D_{\mathrm{CH}} / \mathrm{Hz}$ \\
\hline $16.8 \pm 1.2$ & $11.4 \pm 0.3$ \\
\hline
\end{tabular}

Details on sample preparation, ${ }^{7} \mathrm{Li}$ and CLIP-HSQC parameters are described in the experimental sections 5.1 and 5.2 .

\section{Generation of a comprehensive set of possible molecular structures}

Common structural motifs in metal $(\mathrm{M})$ cyclopentadienide chemistry as found from crystal structures are heteroleptic $\mathrm{CpML}_{\mathrm{x}}\left(\mathrm{L}=\right.$ Ligand) complexes with $\mathrm{x}=1-3$ as well as homoleptic $\mathrm{Cp}_{2} \mathrm{M}$ complexes with varying charges. ${ }^{[17 d, 165-e]}$ Further motifs such as solvent separated ion pairs may exist, but in the case of CpLi in THF the reduced set of structures is reasonable. Corresponding Lewis formulas are depicted in Scheme 4-7. 


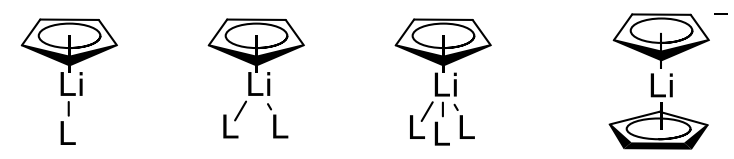

Scheme 4-7: Lewis formulas of possible ligand CpLi complexes and $\left[\mathrm{Cp}_{2} \mathrm{Li}\right]^{-}$.

Starting geometries were built from existing crystal structures of $\mathrm{Cp}$ derivatives. To simplify the geometry optimization of these complexes THF molecules were substituted by $\mathrm{Me}_{2} \mathrm{O}$. This structural cut-down decreases the conformational space while the crucial electronic surrounding of the lithium stays largely unchanged.

The set of structures was geometry optimized by DFT calculations at the B3LYP/def2-TZVP level of theory with ORCA 3.0.3. ${ }^{[154]}$ Stationary points were characterized as minima by computing numerical frequencies.

\section{i) Determination of alignment tensors from RDCs and structural proposals}

The rapid rotation of the Cp ring moiety yields a single RDC. Similar to a methyl group this averaged $\mathrm{RDC}\langle D\rangle$ (attributed to five $\mathrm{C}-\mathrm{H}$ bonds) can be converted into an RDC $D_{\|}$of a virtual bond pointing along the fivefold-symmetry axis of the Cp ring by applying formula (4-1) and an idealized angle of $90^{\circ}$. This is equivalent to a scaling factor of -2 to obtain the virtual RDC. Since only a single RDC can be measured, the alignment tensor must be treated as axially symmetric. According to Table 4-2 this simplification is valid for the symmetries of $\left[\mathrm{CpLi}\left(\mathrm{OMe}_{2}\right)\right],\left[\mathrm{CpLi}\left(\mathrm{OMe}_{2}\right)_{3}\right]$ and $\left[\mathrm{Cp}_{2} \mathrm{Li}\right]^{-}$but requires a more careful analysis of the quasi $C_{2 \mathrm{v}}$ symmetric $\left[\mathrm{CpLi}\left(\mathrm{OMe}_{2}\right)_{2}\right]$ as will be pointed out when appropriate in the following analysis. ${ }^{\mathrm{a}}$

Similar to the alignment tensor determination in section 4.1, the fivefold axis of $\mathrm{Cp}$, i.e. the virtual $\mathrm{C}-\mathrm{H}$ vector $(22.8 \mathrm{~Hz})$, is set in a coordinate system pointing along the $z$ axis. This allows straightforward calculation of values for the alignment tensor as summarized in Table 4-10.

Table 4-10: Alignment tensor elements $A_{\tilde{\mathrm{x}}}, A_{\tilde{\mathrm{y}}}$ and $A_{\tilde{\mathrm{z}}}$ for CpLi in swollen THF/PS (0.4\% DVB) as determined from its RDC. Additionally, the magnitude of these values and the orientation within the molecular frame are represented by arrows with corresponding length, their sign is represented by their color (blue: positive, red: negative).

$\begin{array}{ll}A_{\tilde{\mathbf{x}}} / 10^{-4} & -1.6 \\ A_{\tilde{y}} / 10^{-4} & -1.6 \\ A_{\tilde{\mathrm{z}}} / 10^{-4} & 3.2\end{array}$

Note that the alignment tensor elements are again ordered by increasing magnitude according to conventions. A graphical representation of $\mathbf{A}$ in its PAS is given to illustrate the alignment. The

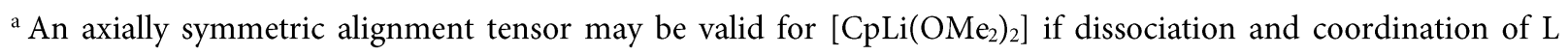
(THF) occurs faster than the molecular rotations. 
orientation of the magnetic field along the Li-Cp bond is in accordance with steric considerations (contrary to the small aromatic molecules discussed in section 4.1). ${ }^{\mathrm{a}}$

\section{Determination of EFGs from complementary techniques}

Absolute values of the EFG can be determined from solid state NMR spectra of quadrupolar nuclei. Subsequent molecular structure determination from X-ray crystallography enables the correlation of EFG values with structural features. In 1998 Johnels, Boman and Edlund conducted a study in which several cyclopentadienide lithium complexes were studied by ${ }^{7} \mathrm{Li}$ solid state NMR. ${ }^{[165 b]}$ Table 4-11 summarizes some of their results:

Table 4-11: CpLi derivatives, added ligand and number of coordinating atoms in parentheses, quadrupolar coupling constants $\chi$ and derived $\left|V_{\tilde{z}}\right|$ and asymmetry parameter $\eta$. Note that the gradient elements are reported in units of a.u. $\left(1\right.$ a.u. $\left.=9.71736 \cdot 10^{21} \mathrm{Vm}^{-2}\right)$. Values taken from reference [165b].

\begin{tabular}{ccccc}
\hline Cp Derivative & Ligand & $\chi / \mathrm{kHz}$ & $\left|V_{\tilde{z}}\right| /$ a.u. & $\eta$ \\
\hline Tris-TMS-CpLi & THF $(1 \mathrm{O})$ & 125 & 0.0133 & 0.1 \\
Tris-TMS-CpLi & Diglyme (2O) & 94 & 0.0100 & 0.1 \\
CpLi & 12 -crown-4 (4O) & 21 & 0.0022 & 0.5 \\
CpLi & PPh $_{4}^{+a}$ & 45 & 0.0048 & 0.3 \\
CpLi & Diglyme $^{\mathrm{a}}$ & 26 & 0.0028 & 0.3 \\
\hline
\end{tabular}

${ }^{a}$ In these cases sandwich complexes are formed.

Most obviously, $\chi$ decreases as the number of oxygen atoms around lithium increases. Also, in the case of sandwich complexes only weak coupling constants are measured (the authors additionally report a "very low" $\chi$ for polymeric CpLi strands). In general, sandwich complexes give only very small quadrupolar coupling constants as the electric field is assumed to be mostly isotropic in complexes with increased hapticity. ${ }^{[162]}$ The EFGs are largely axially symmetric $(\eta \approx 0)$ as can already be anticipated from molecular geometry (slightly higher values for $\eta$ are again usually correlated with lower $\chi$ which indicates increasing spherically symmetric charge distributions). Comparison with crystal structures led to the conclusion that quadrupolar coupling constants "depend on the number and type of donor atoms" rather than on distances and angles. ${ }^{[165 b]}$

This final notion is particularly important as it signals the possibility to transfer results to further $\mathrm{Cp}$ derivatives and different ligands although these are encompassed by (small) structural changes. Hence, the examined system CPLi in THF and the associated structural proposals may show slightly different angles and distances but can nevertheless be directly correlated to the values given in Table 4-11. This correlation of the structural proposals to EFGs with idealized axial symmetry is summarized in Table 412.

\footnotetext{
${ }^{a}$ Note, that a more detailed MD simulation of the alignment of possible aggregates (with different numbers of $\mathrm{L}$ ) would already allow their discrimination by subsequent comparison with experimental alignment. This approach was not further followed here, since, so far, the results in subsection 4.1.1 were not quantitatively matching the experimental benchmark test studies and lithium organics in general have not been very well adapted to molecular dynamics simulations.
} 
Table 4-12: Estimated EFG element $V_{\tilde{z}}$ and asymmetry parameter $\eta$ for $\left[\mathrm{CpLi}\left(\mathrm{OMe}_{2}\right)\right]$, $\left[\mathrm{CpLi}\left(\mathrm{OMe}_{2}\right)_{2}\right]$ $\left[\mathrm{CpLi}\left(\mathrm{OMe}_{2}\right)_{4}\right]$ and $\left[\mathrm{Cp}_{2} \mathrm{Li}\right]^{-}$.

\begin{tabular}{ccc}
\hline Cp Derivative & $\left|V_{\tilde{z}}\right| /$ a.u. & $\eta$ \\
\hline$\left[\mathrm{CpLi}\left(\mathrm{OMe}_{2}\right)\right]$ & 0.013 & 0 \\
{$\left[\mathrm{CpLi}\left(\mathrm{OMe}_{2}\right)_{2}\right]$} & 0.010 & 0 \\
{$\left[\mathrm{CpLi}\left(\mathrm{OMe}_{2}\right)_{4}\right]$} & 0.002 & 0 \\
{$\left[\mathrm{Cp}_{2} \mathrm{Li}\right]^{-}$} & 0.004 & 0 \\
\hline
\end{tabular}

This list lacks the value for a trifold $\mathrm{Me}_{2} \mathrm{O}$ coordination, but it seems reasonable that $\left[\mathrm{CpLi}\left(\mathrm{OMe}_{2}\right)_{3}\right]$ exhibits a quadrupolar coupling constant ranging between $\left[\mathrm{CpLi}\left(\mathrm{OMe}_{2}\right)_{2}\right]$ and $\left[\mathrm{CpLi}\left(\mathrm{OMe}_{2}\right)_{4}\right]$. This dependence on the availability of solid state EFG data is an obvious drawback. Furthermore, information about the orientation of the EFG within the molecular framework cannot be provided by solid state NMR. Yet, this knowledge can be obtained by first principle DFT calculations of the EFG tensor.

$\mathrm{ORCA}^{[154]}$ (for gas phase calculations) or $\mathrm{CASTEP}^{[166]}$ (for solid state calculations) enable a straightforward determination of NMR properties. The B3LYP density functional and Gaussian type basis sets such as $6-311++G^{* *}$ have proven to yield reliable results for the calculation of the EFG of aluminium compounds. In this work the theoretical quadrupolar coupling $\chi$ of trimesityl aluminum $\left(\mathrm{Al}(\mathrm{Mes})_{3}\right)$ was reproduced utilizing ORCA 3.0.3 ${ }^{[154]}$ and the fgrad keyword (for EFG calculation) at the B3LYP/6-311++ $\mathrm{G}^{* *}$ level ${ }^{[167]}$ of theory. This procedure was then repeated for the structural proposals $\left[\mathrm{CpLi}\left(\mathrm{OMe}_{2}\right)\right],\left[\mathrm{CpLi}\left(\mathrm{OMe}_{2}\right)_{2}\right],\left[\mathrm{CpLi}\left(\mathrm{OMe}_{2}\right)_{3}\right]$ and $\left[\mathrm{Cp}_{2} \mathrm{Li}\right]^{-}$. Resulting values are summarized in Table 4-13. Additionally, the table includes graphical tensor representations that illustrate the EFG orientation and asymmetry.

Table 4-13: Theoretical values for $\left|V_{\tilde{z}}\right|$ and $\eta$ and their graphical representation by spherical polar plots ${ }^{[162 b]}$ for $\mathrm{Al}(\mathrm{Mes})_{3},\left[\mathrm{CpLi}\left(\mathrm{OMe}_{2}\right)\right],\left[\mathrm{CpLi}\left(\mathrm{OMe}_{2}\right)_{2}\right]\left[\mathrm{CpLi}\left(\mathrm{OMe}_{2}\right)_{3}\right]$ and $\left[\mathrm{Cp}_{2} \mathrm{Li}\right]^{-}$.

\begin{tabular}{cccc}
\hline$V_{\tilde{z}} \mid$ & $\eta$ & \\
\hline $\left.\mathrm{CpLi}\left(\mathrm{OMe}_{2}\right)\right]$ & 0.015 & 0.5 & \\
{$\left[\mathrm{CpLi}\left(\mathrm{OMe}_{2}\right)_{2}\right]$} & 0.018 & 0.9 & \\
{$\left[\mathrm{CpLi}\left(\mathrm{OMe}_{2}\right)_{3}\right]$} & 0.005 & 0.4 & \\
{$\left[\mathrm{Cp}_{2} \mathrm{Li}\right]^{-}$} & 0.005 & 0.2 & \\
\hline
\end{tabular}

The computational values $\left|V_{\tilde{z}}\right|$ for $\left[\mathrm{CpLi}\left(\mathrm{OMe}_{2}\right)\right]$ and $\left[\mathrm{Cp}_{2} \mathrm{Li}\right]^{-}$agree well with experimental data provided by Edlund et al. but the asymmetry is larger than anticipated. In both cases the axial 
component is largely oriented along the $\mathrm{Li}-\mathrm{Cp}$ bond. $\left[\mathrm{CpLi}\left(\mathrm{OMe}_{2}\right)_{3}\right]$ gives a reasonable, i.e. small, $\left|V_{\tilde{z}}\right|$ similar to $\left[\mathrm{Cp}_{2} \mathrm{Li}\right]^{-}$. This is in accordance with the trend provided by experimental data in Table 4-12 and, therefore, this value can, with reservations, be further evaluated. This is different for $\left[\mathrm{CpLi}\left(\mathrm{OMe}_{2}\right)_{2}\right]$ which additionally exhibits EFG tensor elements that are strongly tilted away from the $\mathrm{Li}-\mathrm{Cp}$ bond. The calculated values for $\left[\mathrm{CpLi}\left(\mathrm{OMe}_{2}\right)_{2}\right]$ largely exceed the experimental $\left|V_{\tilde{z}}\right|$ and $\eta$. This may be explained from the effects of crystal packing and therefore one should very carefully interpret proceed with the following discussions related to this case..

\section{Calculation of theoretical RQCs from alignment tensor and EFG and assessment of the agreement between calculated and experimental RQCs}

Three major simplifications are made to obtain preliminary results:

i) Principal axes belonging to the axial components of $\mathbf{A}$ and $\mathbf{V}\left(A_{\tilde{\mathrm{z}}}\right.$ and $\left.V_{\tilde{\mathrm{Z}}}\right)$ are collinear and lie within the $\mathrm{Li}-\mathrm{Cp}$ bond axis.

ii) $\quad$ V is axially symmetric

iii) $\quad \mathbf{A}$ is axially symmetric

According to these assumptions formula (1-13) can be simplified to equation (4-5) for ${ }^{7} \mathrm{Li}$ RQCs.

$$
v_{\mathrm{rq}}=\frac{3 e Q}{4 h} A_{\tilde{\mathbf{z}}} V_{\tilde{\mathbf{z}}}
$$

The resulting theoretical RQCs $\left|v_{\mathrm{rq}, \mathrm{cal}}\right|$ for the hypothetical CpLi structures are summarized in Table 414.

Table 4-14: Alignment tensor element $A_{\tilde{z}}$, EFG tensor element $\left|V_{\tilde{z}}\right|$ and theoretical quadrupolar couplings $\mid v_{\text {rq }}$ call $\mid$ for $\left[\mathrm{CpLi}\left(\mathrm{OMe}_{2}\right)\right],\left[\mathrm{CpLi}\left(\mathrm{OMe}_{2}\right)_{2}\right]\left[\mathrm{CpLi}\left(\mathrm{OMe}_{2}\right)_{3}\right]$ and $\left[\mathrm{Cp}_{2} \mathrm{Li}\right]^{-}$and experimental value $\left|v_{\text {rq,exp }}\right|$.

\begin{tabular}{ccccc}
\hline Cp Derivative & $A_{\tilde{z}} / 10^{-4}$ & $\left|V_{\tilde{z}}\right| /$ a.u. & $\mid v_{\text {rq } q \text {,ald }} / \mathrm{Hz}$ & $\left|v_{\text {rq }, \text { exp }}\right| / \mathrm{Hz}$ \\
\hline$\left[\mathrm{CpLi}\left(\mathrm{OMe}_{2}\right)\right]$ & & 0.013 & 29.4 & \\
{$\left[\mathrm{CpLi}\left(\mathrm{OMe}_{2}\right)_{2}\right]$} & 3.2 & 0.010 & 22.6 & 16.8 \\
{$\left[\mathrm{CpLi}\left(\mathrm{OMe}_{2}\right)_{3}\right]^{\mathrm{a}}$} & & 0.005 & 11.3 & \\
{$\left[\mathrm{Cp}_{2} \mathrm{Li}^{-}\right.$} & & 0.004 & 9.00 & \\
\hline
\end{tabular}

${ }^{\text {a }}$ Value for $\left|V_{\tilde{z}}\right|$ is taken from DFT calculations.

The difference between values $\left|v_{\mathrm{rq}, \text { cal }}\right|$ and $\left|v_{\mathrm{rq}, \text { exp }}\right|$ for $\left[\mathrm{CpLi}\left(\mathrm{OMe}_{2}\right)\right]$, [CpLi( $\left.\left(\mathrm{OMe}_{2}\right)_{2}\right]\left[\mathrm{CpLi}\left(\mathrm{OMe}_{2}\right)_{3}\right]$ and $\left[\mathrm{Cp}_{2} \mathrm{Li}\right]^{-}$is additionally illustrated in Figure 4-18. 


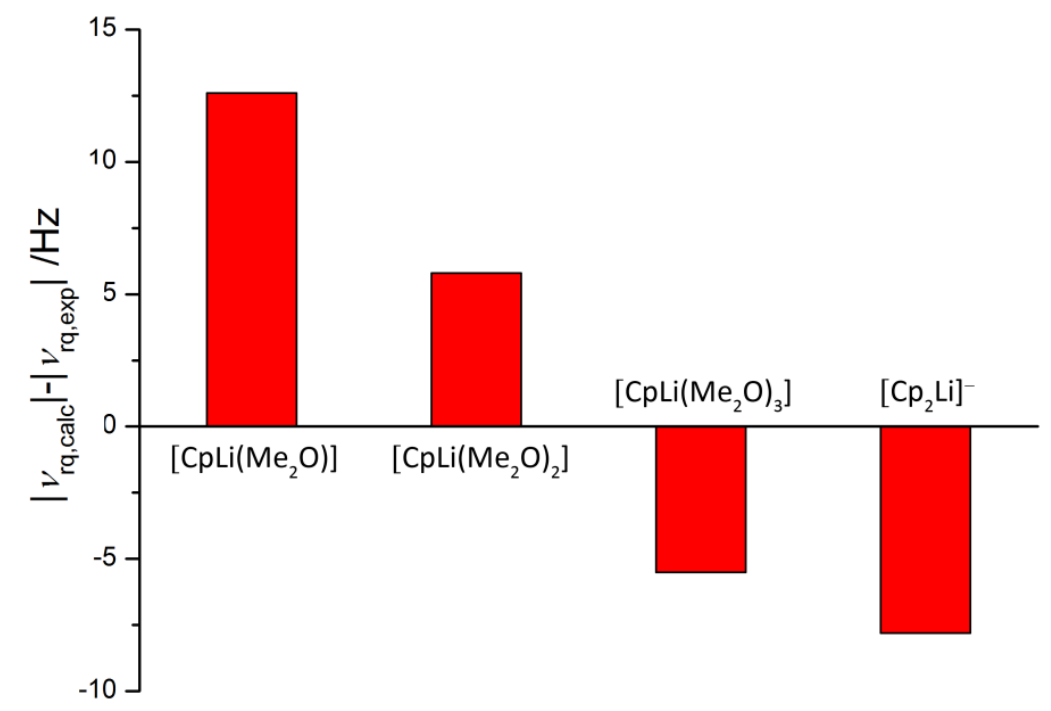

Figure 4-18: Difference between values $\left|v_{r q, \text { cal }}\right|$ and $\left|v_{r q, \text { exp }}\right|$ for $\left[\mathrm{CpLi}\left(\mathrm{OMe}_{2}\right)\right]$, $\left[\mathrm{CpLi}\left(\mathrm{OMe}_{2}\right)_{2}\right]\left[\mathrm{CpLi}\left(\mathrm{OMe}_{2}\right)_{3}\right]$ and $\left[\mathrm{Cp}_{2} \mathrm{Li}\right]^{-}$.

It must be emphasized that differentiation between structures must, so far, be based on matching absolute values, since only the magnitude of the experimental RQC can be measured. This has to be addressed in future attempts to develop the RQC structure verification method. However, a quantitative match is a strong indicator and supports complementary methods such as DOSY MW determination. Indeed, [CpLi(THF $\left.)_{2}\right]$ has been confirmed to be the predominant species by DOSY. ${ }^{[165 a]}$ This result can be confirmed in the following discussion:

$\left[\mathrm{CpLi}\left(\mathrm{OMe}_{2}\right)\right]$ and $\left[\mathrm{Cp}_{2} \mathrm{Li}\right]^{-}$show large deviations from the experimental coupling. Additionally, the assumptions made about axial symmetry of $\mathbf{A}$ and $\mathbf{V}$ are most valid for both structures. Hence, they are far less probable than $\left[\mathrm{CpLi}\left(\mathrm{OMe}_{2}\right)_{2}\right]$ or $\left[\mathrm{CpLi}\left(\mathrm{OMe}_{2}\right)_{3}\right]$. In turn, one cannot unambiguously differentiate between the latter proposals as both deviate by $\sim 6 \mathrm{~Hz}$ from the experimental value. A more nuanced inspection of the initial approximations is therefore required for $\left[\mathrm{CpLi}\left(\mathrm{OMe}_{2}\right)_{2}\right]$ and $\left[\mathrm{CpLi}\left(\mathrm{OMe}_{2}\right)_{3}\right]$.

Especially, assumption i) (collinear tensor elements $A_{\tilde{Z}}$ and $V_{\tilde{z}}$ ) is insufficient since results from DFT calculations (Table 4-13) indicate an orthogonal orientation of the principal axes belonging to the axial components (Figure 4-19).
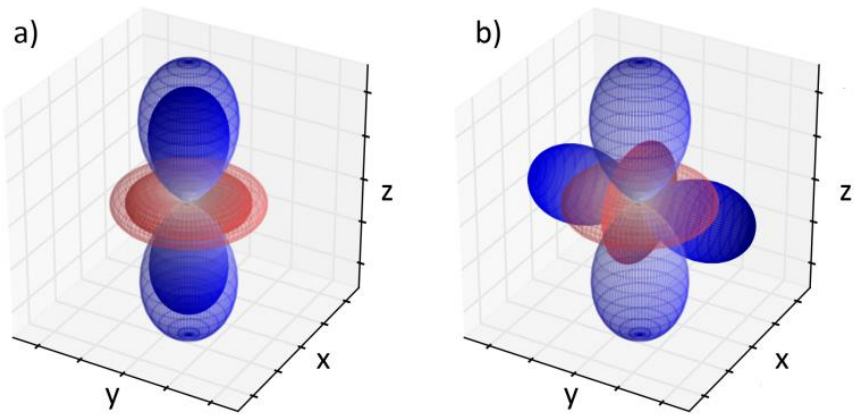

Figure 4-19: Two possible orientations of the alignment tensor (bigger, lighter) and the EFG tensor (smaller, darker) represented by spherical plots. a) Initial assumption that axial components are parallel to each other. b) Adapted assumption that axial components are orthogonal to each other. 
According to the original formula (1-13), the tilt towards orthogonal orientation will result in smaller sums and the theoretical $\left|v_{\text {rq,all }}\right|$ will decrease. This leads to a bigger disagreement between theory and experiment for a hypothetical aggregate $\left[\mathrm{CpLi}\left(\mathrm{OMe}_{2}\right)_{3}\right]$ but the hypothetical RQC for [CpLi( $\left.\left(\mathrm{OMe}_{2}\right)_{2}\right]$ will approach the experimental value. Retaining assumptions ii) and iii) (axially symmetric tensors $\mathbf{A}$ and V), the RQCs can be calculated for the adapted EFG tensor orientation (Figure 4-19 b). The appropriate Cartesian tensor elements are summarized in Table 4-15.

Table 4-15: Adapted $\mathbf{V}$ and $\mathbf{A}$ values in a shared Cartesian coordinate system in which the $\mathrm{Li}-\mathrm{Cp}$ bond is aligned along $\mathrm{z}$ and the $\mathrm{O}-\mathrm{Li}-\mathrm{O}$ bonds are set within the $\mathrm{x}-\mathrm{z}$ plane.

\begin{tabular}{cccc}
\hline $\mathrm{i}=\mathrm{j}$ & $V_{\mathrm{i}}\left[\mathrm{CpLi}\left(\mathrm{OMe}_{2}\right)_{2}\right] /$ a.u. $^{\mathrm{a}}$ & $V_{\mathrm{i}}\left[\mathrm{CpLi}\left(\mathrm{OMe}_{2}\right)_{3}\right] /$ a.u. $^{\text {a }}$ & $A_{\mathrm{i}} / 10^{-4}$ \\
\hline $\mathrm{x}$ & -0.005 & -0.0025 & -1.6 \\
$\mathrm{y}$ & 0.010 & 0.0050 & -1.6 \\
$\mathrm{z}$ & -0.005 & -0.0025 & 3.2 \\
\hline
\end{tabular}

${ }^{a}$ Note the distinction from the ordering (naming) convention of the EFG tensor.

In this coordinate system, the $\mathrm{Li}-\mathrm{Cp}$ bond is aligned along $z$ and the $\mathrm{O}-\mathrm{Li}-\mathrm{O}$ bonds are set within the $\mathrm{x}$ $\mathrm{z}$ plane. This leads to adapted theoretical $\left|v_{\text {rq } q \text { cal }}\right|$ as presented in Table 4-16.

Table 4-16: Values $\left|v_{\mathrm{rq}, \mathrm{cal}}\right|$ as obtained from an orthogonal orientation of axially symmetric $\mathbf{A}$ and $\mathbf{V}$ for $\left[\mathrm{CpLi}\left(\mathrm{OMe}_{2}\right)_{2}\right]$ and $\left[\mathrm{CpLi}\left(\mathrm{OMe}_{2}\right)_{3}\right]$.

\begin{tabular}{lcc}
\hline & $\mid v_{\text {rq, cal }} / / \mathrm{Hz}$ & $\left|v_{\text {rq,exp }}\right| / \mathrm{Hz}$ \\
\hline$\left[\mathrm{CpLi}\left(\mathrm{OMe}_{2}\right)_{2}\right]$ & $11.3 \mathrm{~Hz}$ & 16.8 \\
{$\left[\mathrm{CpLi}\left(\mathrm{OMe}_{2}\right)_{3}\right]$} & $5.6 \mathrm{~Hz}$ & \\
\hline
\end{tabular}

In accordance with the qualitatively anticipated decrease of theoretical $\left|v_{\mathrm{rq} \text { cal }}\right|$, the result for $\left[\mathrm{CpLi}\left(\mathrm{OMe}_{2}\right)_{2}\right]$ is closer to the experimental value, yet, it is not a particularly good match. Application of the EFG tensor values from DFT calculations (Table 4-13) does not improve the agreement $\left(\left|v_{\mathrm{rq}, \mathrm{cal}}\right|=2.7 \mathrm{~Hz}\right)$. Simplification iii) can be further examined by decreasing the symmetry of $\mathbf{A}$. In any case, $A_{\mathrm{z}}$ is constant $\left(3.2 \cdot 10^{-4}\right)$, but $A_{\mathrm{x}}$ can range between 0 and $-A_{\mathrm{z}}$ while $A_{\mathrm{y}}$ changes dependently according to $A_{\mathrm{y}}=-A_{\mathrm{z}}-A_{\mathrm{x}}$ (Table 4-17).

Table 4-17: Adapted $\mathbf{V}$ and changing $\mathbf{A}$ values in a shared Cartesian system, in which $\mathrm{Li}-\mathrm{Cp}$ bond is aligned along $z$ and the $\mathrm{O}-\mathrm{Li}-\mathrm{O}$ bonds are set within the $\mathrm{x}-\mathrm{z}$ plane.

\begin{tabular}{ccc}
\hline $\mathrm{i}=\mathrm{j}$ & $V_{\mathrm{i}}\left[\mathrm{CpLi}\left(\mathrm{OMe}_{2}\right)_{2}\right] /$ a.u. $^{\mathrm{a}}$ & $A_{\mathrm{i}} / 10^{-4}$ \\
\hline $\mathrm{x}$ & -0.005 & $0 \rightarrow-3.2$ \\
$\mathrm{y}$ & 0.010 & $-3.2 \rightarrow 0$ \\
$\mathrm{z}$ & -0.005 & 3.2 \\
\hline
\end{tabular}

${ }^{a}$ Note the distinction from the ordering (naming) convention of the EFG tensor. 
This leads to new values $\left|v_{\mathrm{rq}, \mathrm{cal}}\right|$. The corresponding differences $\left\|v_{\mathrm{rq}, \mathrm{cal}}|-| v_{\mathrm{rq} \text { exp }}\right\|$ for varying $A_{\mathrm{x}}$ is illustrated in Figure 4-20.

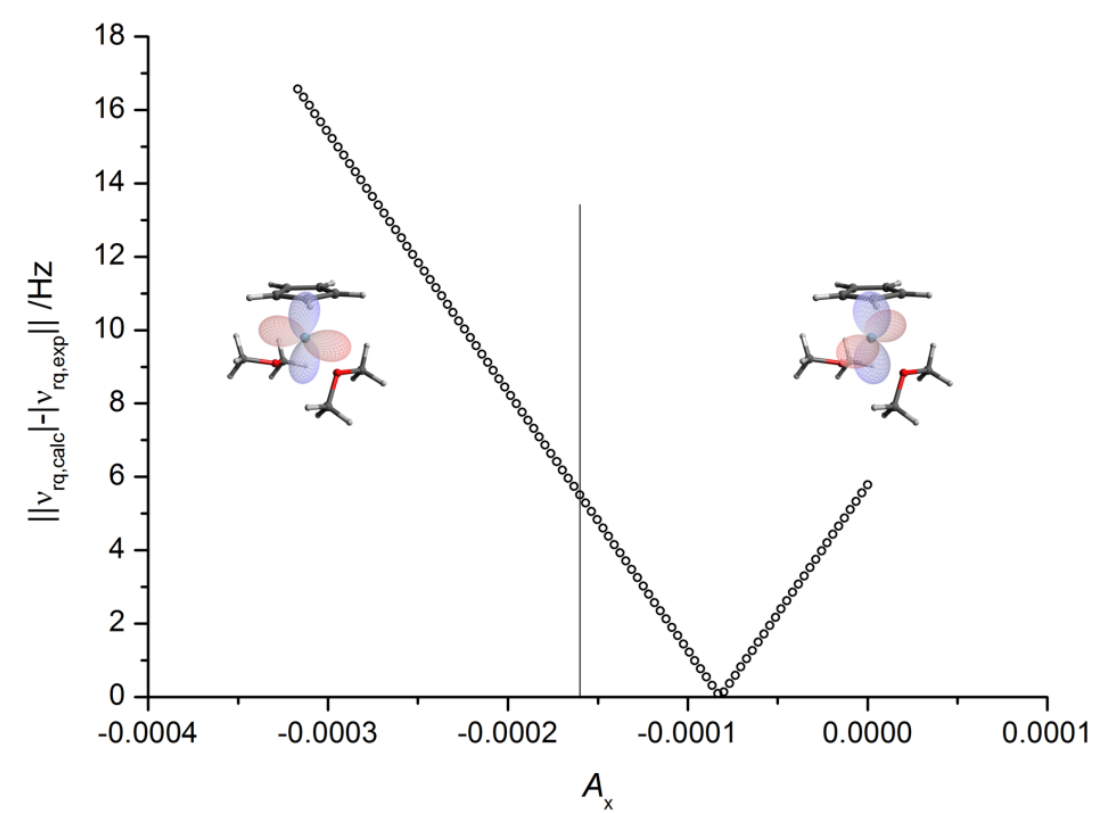

Figure 4-20: Resulting values $\| v_{\mathrm{rq}, \mathrm{cal}}|-| v_{\mathrm{rq} \text { exp }}||$ for $\left[\mathrm{CpLi}\left(\mathrm{OMe}_{2}\right)_{2}\right]$ and varying $A_{\mathrm{x}}$. The vertical line marks the axially symmetric alignment tensor with $A_{\mathrm{y}}=A_{\mathrm{x}}=-1 \cdot 6 \cdot 10^{-4}$. Additionally, the boundary alignment tensors for $A_{\mathrm{x}}=-A_{\mathrm{z}}$, $A_{\mathrm{y}}=0$ (left) and $A_{\mathrm{x}}=0, A_{\mathrm{y}}=-A_{\mathrm{z}}$ (right) are illustrated by polar plots.

Recall, that a more negative alignment tensor element indicates a less preferred orientation of the related axis along the magnetic field/elongated pore. According to the obvious spatial dimensions of the molecule, the axis that is perpendicular to the $\mathrm{O}-\mathrm{Li}-\mathrm{O}$ plane is least likely aligned with the magnetic field vector. This corresponds to the right side of Figure 4-20. Hence, under more realistic assumptions, the theoretical $\left|v_{\text {rq, cal }}\right|$ approaches the experimental value.

In summary, the results suggest that $\left[\mathrm{CpLi}(\mathrm{THF})_{2}\right]$ is the correct structure of $\mathrm{CpLi}$ in solutions of THF. Furthermore, Figure 4-18 shows, that RQCs can unambiguously resolve issues such as exemplified in Scheme 4-6: The two structures $[\mathrm{CpLi}(\mathrm{THF})]$ and $\left[\mathrm{Cp}_{2} \mathrm{Li}\right]^{-}$have very similar weight and thus cannot be distinguished by DOSY but give very different RQCs which can be quantitatively matched to structural proposals. Moreover, a detailed estimation of the EFG by first principles enables a more detailed application of equation (1-13) taking account of the relative orientation of the tensors. As such, structure verification by RQCs is a valuable tool for the examination of molecules bearing quadrupolar nuclei.

Finally, note that according to formula (1-13) measurement of an arbitrary molecule in five different alignment media could provide five different alignment tensors which do not linearly depend on each other (this number is decreased if the position of the quadrupolar atom coincides with symmetry elements). In this case, a linear equation system would provide the full set of EFG tensor elements in the chosen coordinate frame (typically the alignment tensor PAS). The full EFG and information on the asymmetry parameter $\eta$ would per se provide subtle structural information. 


\subsection{Summary and Outlook Anisotropic NMR Spectroscopy}

The studies described above show that anisotropic NMR spectroscopy is an effective tool to

i) observe and understand alignment phenomena in uniaxial environments,

ii) determine the configuration and conformation of small, rigid molecules,

iii) determine the aggregation state of organometallic compounds.
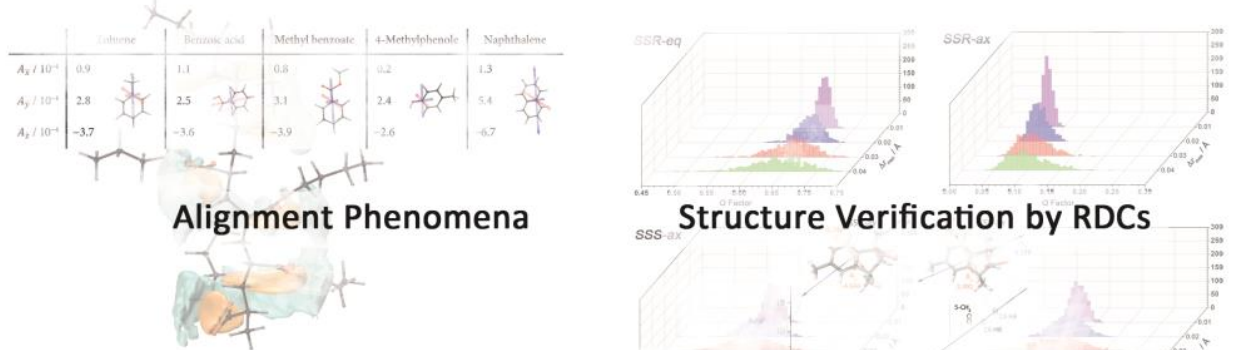

Structure Verification by RDCs

Anisotropic NMR Spectroscopy

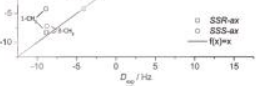

Structure Verification by RQCs

Figure 4-21: Anisotropic NMR spectroscopy and its applications: i) Alignment of small molecules in stretched polymer gels. ii) Structure determination of small rigid molecules by RDCs. iii) Structure determination of organometallic compounds by RQCs.

Alignment phenomena were observed by RDC measurements of small aromatic molecules in PS and PBA. These results showed surprising alignment phenomena which are characterized by a mismatched orientation of molecules considering their spatial extensions and the pore geometry. An orthogonal orientation (between longest pore and longest molecule axis) was found for toluene, 4-methylphenol and naphthalene in PS (Figure 4-22) and for benzoic acid and 4-methylphenol in PBA.

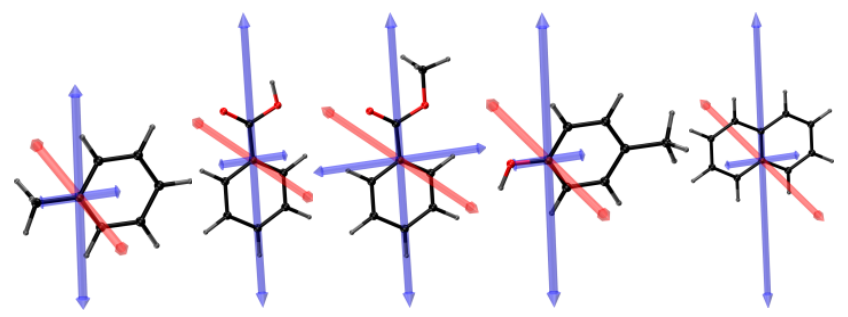

Figure 4-22: Alignment tensor representation of small aromatic molecules in PS. From left to right: toluene, benzoic acid, methyl benzoate, 4-methylpenol and naphthalene.

This provides evidence that the steric orientation (parallel orientation of longest axes) which is the basic assumption for a range of first principle alignment tensor prediction methods (Tramite ${ }^{[7]]}$, PALES ${ }^{[81]}$ ) must always be questioned for small molecules. 
The alignment phenomena were rationalized by an interplay of repulsive and attractive interactions between the alignment medium and the aligned solute: The repulsive interaction induces an, intuitive, parallel orientation since the elongated polymer strains function as barriers. The attractive interaction can be further described as a rather unspecific interaromatic interaction in the case of PS and as a hydrogen bridge bonds in the case of PBA.

These alignment phenomena were qualitatively reproduced by MD simulations, which will become an increasingly important tool for alignment tensor prediction, considering the failure of traditional (sterical) prediction methods in cases like the ones described above. ${ }^{[80,132]}$ In contrast to traditional prediction methods, MD simulations can additionally account for dynamic features such as conformational changes. Finally, the results on small molecule alignment showed that the solvent effects of anisotropic media cannot be neglected. Unlike in a former study, the observations clearly showed that anisotropic media can have profound impact on solutes. ${ }^{[159]}$ E.g. interactions leading to the orthogonal and parallel orientation may both be present within different rotatable moieties of a molecule and hence change the conformational landscape of the observed substance within uniaxial environments. This has to be considered in studies concerning small molecules in polymers and may again be best addressed through comparative MD simulations (alignment) as well as high level theoretical calculations (population of conformers).

Structure verification by RDCs has been successfully applied to two synthetic $\beta$-amino acid esters both resulting from the same catalytic $\mathrm{C}-\mathrm{H}$ bond activation cycle. In accordance with the proposed reaction mechanism, the cis configuration of the amine and ester were unambiguously determined as the true structure. Furthermore, RDC-based conformation analysis showed mono-populated conformations with a switch from the pseudo-equatorially oriented ester to a pseudo-axial conformation upon the substitution of a hydrogen atom by a methyl group at the $\mathrm{C} 3$ ring position. This change is remarkable and may play a vital role in the discussion of these amino acid esters as $\beta$-peptide building blocks with varying secondary structures.

Furthermore, the traditional $Q$ factor for the assessment of structural proposal was shown to be insufficient in special cases, but the addition of structural noise emerged as a useful further criterion that complements the traditional approach: The addition of structural noise to critical $\mathrm{C}-\mathrm{H}$ and $\mathrm{C}-\mathrm{Me}$ bonds generated a thermally accessible ensemble of molecules and enables the generation of $Q$ factor distributions. The shape of the distribution for the true structure (asymmetric, steep) differs from the distributions of wrong structures (symmetric, broad). In the case of only few RDCs, it is possible that wrong structural proposals accidentally give a sufficiently good $Q$ factor. These wrong structures behave stochastically and hence are well described by Gaussian distributions. In contrast, true structures show a $Q$ factor minimum i.e. an asymmetric, narrower distribution. The validation process by structural noise can be easily implemented and adds a straightforward and very reliable figure to amend the traditional $Q$ factor, which will become especially useful in automated structure elucidation by algorithms. ${ }^{[168]}$ Structure verification by RQCs was finally presented as a novel approach to derive structural information on lithium organic compounds. This technique fundamentally relies on the alignment tensor determination from RDCs and the a priori determination of the EFG either by first principles (DFT methods $)^{[162 b]}$ or solid state NMR spectroscopy ${ }^{[61 c]}$. While this method is far from being fully developed 
i.e. various technical issues, in particular the sign determination of experimental RQCs, have to be addressed in future studies, its potential was shown in a case study on CpLi in THF. The combination of RQC/RDC and theoretical calculations of the EFG led to $\left[\mathrm{CpLi}(\mathrm{THF})_{2}\right]$ as the solution structure of CpLi in THF. This is in accordance with DOSY MW methods.
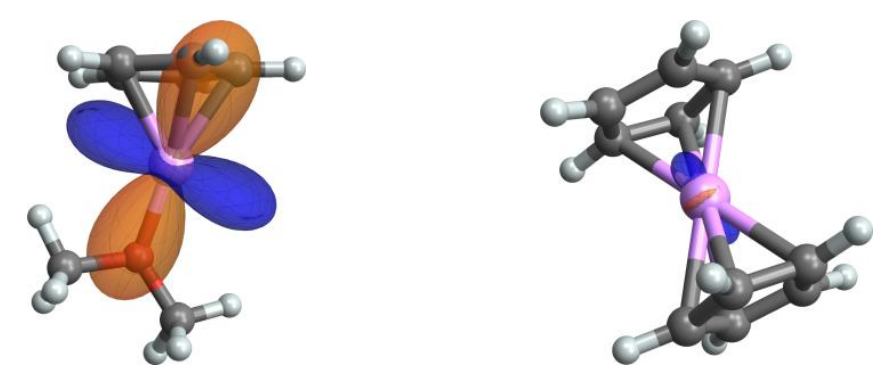

Figure 4-23: Spherical polar plot EFG tensor representation around the lithium atom in hypothetical $\left[\mathrm{CpLi}\left(\mathrm{OMe}_{2}\right)\right]$ (left) and $\left[\mathrm{Cp}_{2} \mathrm{Li}\right]^{-}$(right).

It is important to mention that RQCs are, in contrast to DOSY, also able to clarify the distinction between molecular aggregates with small variations in $M W$. For example the hypothetical [CpLi(THF)] and $\left[\mathrm{Cp}_{2} \mathrm{Li}\right]^{-}$have very similar MW but can be clearly distinguished by the size of the reproduction of RQCs through theoretical EFGs and experimental RDCs. 


\section{EXPERIMENTAL PART}

\subsection{Sample Preparation}

\section{Synthesis and Handling of Air- and Moisture-Sensitive Compounds}

All experimental standard Schlenk procedures took place under inert gas atmosphere (purified, dry argon) in a glove box. Glassware was dried at $130^{\circ} \mathrm{C}$, assembled hot and cooled down under vacuum. All solvents were dried and distilled over sodium-potassium alloy and finally degassed (three pump-freeze cycles) prior to use. Chemicals were purchased as commercially available products or synthesized according to known literature. $n$ BuLi was kindly donated by Rockwood Lithium.

Methyl-1-\{(4-methoxyphenyl)amino\}-1,5-dimethyl-2,3-dihydro- $1 H$-indene-2-carboxylate

Formula:

$\mathrm{C}_{20} \mathrm{H}_{23} \mathrm{NO}_{3}$

Molecular weight:

$339.2 \mathrm{~g} / \mathrm{mol}$<smiles>COC(=O)[C@]1(Nc2ccc(OC)cc2)C(=O)[C@H](C)c2ccc(C)cc21</smiles>

Methyl-1-\{(4-methoxyphenyl)amino\}-1,3,5-trimethyl-2,3-dihydro- $1 H$-indene-2-carboxylate

Formula:

$\mathrm{C}_{21} \mathrm{H}_{25} \mathrm{NO}_{3}$

Molecular weight:

$367.2 \mathrm{~g} / \mathrm{mol}$<smiles>COC(=O)C1(C)C(=O)c2cc(C)ccc2N1c1ccc(OC)cc1</smiles>

The indane compounds were synthesized by Liu and Zell from the Ackermann group and then employed as obtained. Experimental characterization has already been performed in reference [148].

Cyclopentadienide-lithium
Formula:
$\mathrm{C}_{5} \mathrm{H}_{5} \mathrm{Li}$
Molecular weight:
$72.0 \mathrm{~g} / \mathrm{mol}$

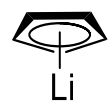

CpLi was synthesized by Bachmann from the Stalke group and then employed as obtained. Experimental characterization has already been performed in reference [165a]. 


\section{Preparation of Polymer Sticks}

Styrene, DVB (industrial grade, $\sim 80 \% m$-DVB), butyl acrylate and 1,4-butanediol diacrylate were separated from inhibitors by flash column chromatography through neutral aluminium oxide $(50 \mathrm{~g})$. Mixtures of DVB in styrene and 1,4-butanediol diacrylate in butyl acrylate were prepared according to the desired volume percentage (0.2\%-0.5\%). Under argon atmosphere, these monomer/linker solutions were degassed (three pump-freeze cycles) and filled into glass tubes sealed at one end $(10 \mathrm{~cm}$ length, $3.8 \mathrm{~mm}$ inner diameter). These tubes were then heated to $115^{\circ} \mathrm{C}$ over three days to accomplish the polymerization.

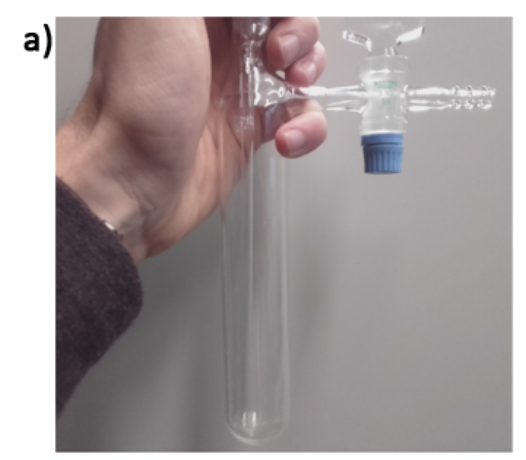

b)
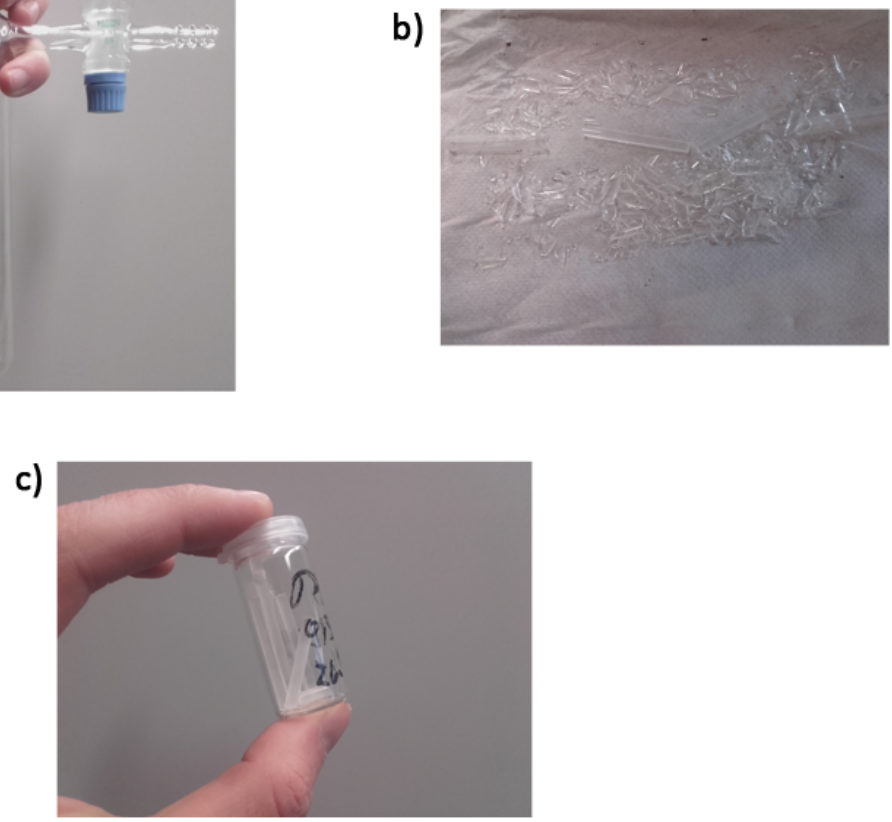

Figure 5-1: Preparation of the polymer sticks: a) Filling of glass tubes with mixtures of PS and DVB under inert gas atmosphere within a Schlenk tube and subsequent heating at $115^{\circ} \mathrm{C}$ over three days. b) Careful breaking of the glass tubes gives the ready-to-use polymer sticks (c).

Note that swollen samples of polymers were usually shimmed on the FID i.e. the FID area was maximized by manual shim routines. 


\section{Observation of Reactions}

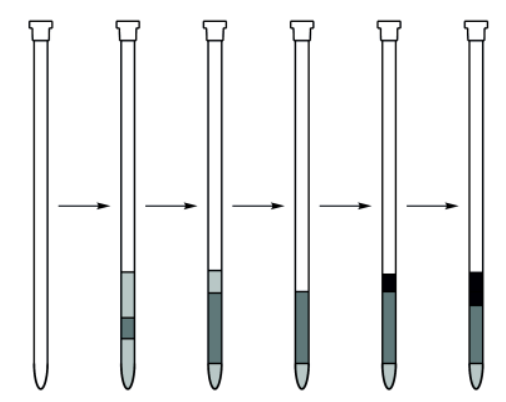

Scheme 5-1: Basic steps of sample preparation: 1. A crosslinked polymer is immersed in a solution which contains the reactive (lithium organic) species. 2. The polymer is swollen/equilibrated over a sufficiently long time period. This can be evaluated through homogeneous signal intensities via SSE as shown by Pöppler et al. 3. The supernatant solution above the polymer is removed. 4. A solution containing a further substrate is added on top of the polymer and diffuses into the gel. 5 . This is then followed by SSE over an appropriate time period.

Due to the high reactivity of organolithium reagents and to prevent rapid convection and/or diffusion out of the active sample volume, $n \mathrm{BuLi}$ was first diffused into a PS gel matrix, and all steps of the preparation were carried out strictly under argon atmosphere. For this purpose, a solution of $n \mathrm{BuLi}$ in $n$-hexane $(0.4 \mathrm{ml}, 1.9 \mathrm{M}, 0.74 \mathrm{mmol})$ was concentrated in vacuo and toluene- $d_{8}(0.40 \mathrm{ml})$ was added. This solution was then transferred into a $5 \mathrm{~mm}$ NMR tube, and a cylindrical PS stick $(10 \times 3.8 \mathrm{~mm}$, crosslinked through 0.2 vol\% divinylbenzene) was immersed in the solution approximately $1 \mathrm{~cm}$ above the bottom of the tube. After 7 days (the PS stick had swollen to a length of $\sim 3 \mathrm{~cm}$ ) the supernatant $n \mathrm{BuLi} /$ toluene solution above the polymer was removed and replaced by a solution of PMDTA $(0.12 \mathrm{ml}$, $0.60 \mathrm{mmol})$ in toluene- $d_{8}(0.15 \mathrm{ml})$. Slice-selective ${ }^{1} \mathrm{H}$ and ${ }^{7} \mathrm{Li}$ NMR measurements started after $\sim 3 \mathrm{~h}$ and went on for three days.

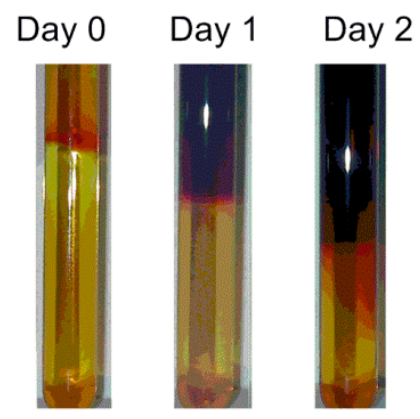

Figure 5-2: Visual tracing of the PMDTA diffusing into the $n \mathrm{BuLi} / \mathrm{PS}$ gel over the course of three days. 


\section{Single-Shot Titration}

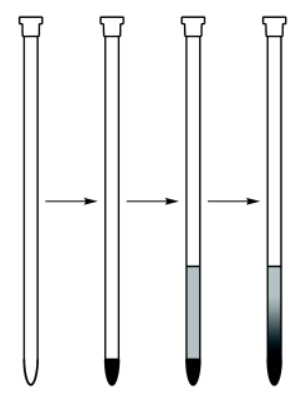

Scheme 5-2: Basic steps of sample preparation: 1. The first compound (neat) is filled into the NMR tube. 2. The second compound, immersed in deuterated solvent, is carefully added on top of the first compound. The compound at the bottom then diffuses into the solution above. 3. This can be readily observed through SSE.

12-crown-4 (50 $\mu \mathrm{L}, 3.1 \mathrm{mmol}$, m.p. $\left.16^{\circ} \mathrm{C}\right)$ was filled into a standard $5 \mathrm{~mm} \mathrm{NMR}$ tube and cooled to $5^{\circ} \mathrm{C}$. Then a solution of $\mathrm{LiClO}_{4}(24 \mathrm{mg}, 2.3 \mathrm{mmol})$ in acetonitrile-d $3(0.45 \mathrm{ml})$ was layered on top of the solid ether. This procedure prevents initial mixing of both components prior to the measurements. Inside the NMR magnet $\left(25^{\circ} \mathrm{C}\right.$, standing tube) the ether melts and slowly diffuses into the $\mathrm{LiClO}_{4}$ solution resulting in a smooth concentration gradient along the tube axis. Approximately 3,6 and $9 \mathrm{~h}$ after sample preparation slice-selective ${ }^{1} \mathrm{H}$ and ${ }^{7} \mathrm{Li}$ NMR spectra were recorded. 


\section{Qualitative Chromatography}

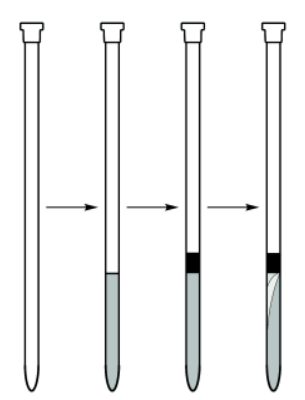

Scheme 5-3: Basic steps of sample preparation: 1. The stationary phase is prepared within the NMR tube. 2. A mixture of compounds to separate is added on top of the stationary phase. 3 . The compounds start diffusing into the mixture at different rates. 4 . The diffusion progress can be readily observed through SSE.

As a case study the separation of amino acids L-Asp acid, L-His, L-Tyr and L-Val within agar hydrogel was examined. This gel matrix has lately been described as a beneficial diffusion controlling medium in an elegant titration experiment by Mitrev et al. Agar $(7.8 \mathrm{mg}$, $2 \mathrm{wt} \%)$ was immersed in $\mathrm{D}_{2} \mathrm{O}(0.35 \mathrm{ml})$ in an NMR tube, and the suspension was heated until a homogeneous gel resulted. A mixture of L-aspartic acid (asp, $7.3 \mathrm{mg}$ ), L-His (his, $8.3 \mathrm{mg}$ ), L-Tyr (tyr, $8.0 \mathrm{mg}$ ) and L-Val (val, $6.5 \mathrm{mg}$ ) in slightly alkaline $\mathrm{D}_{2} \mathrm{O}(0.15 \mathrm{ml})$ was added on top of the gel sample and SSE spectra (19 slices, $1 \mathrm{~mm}$ thickness each) were recorded immediately afterwards as well as 2, 6 and $10 \mathrm{~h}$ later. This procedure was repeated for gel samples with varying amounts of agar weight-percentages ( $3 \mathrm{wt} \%$ and $5 \mathrm{wt} \%$ ). 


\section{Anisotropic NMR Spectroscopy}

In general, about $20 \mathrm{mg}$ of the target compound were dissolved in an appropriate deuterated solvent (small molecules: $\mathrm{CD}_{2} \mathrm{Cl}_{2}$, indanes: $\mathrm{CDCl}_{3}$, lithium-compounds: THF- $d_{8}$ ). Alignment of the compounds was achieved by strain-induced alignment in a gel $(S A G)^{[75 b, 91,95,169]}$ : Either a cylindrical PS stick $(10 \times 3.8 \mathrm{~mm}$, crosslinked through 0.3 vol\% divinylbenzene $)$ or a cylindrical PBA stick $(10 \times 3.8 \mathrm{~mm}$, crosslinked through $0.6 \mathrm{vol} \%$ 1,4-butanediol diacrylate) was immersed in the isotropic solutions. Swelling/equilibration over several days (small molecules: three days for PBA and four days for PS, indanes: four days, lithium compounds: eight days) led to sufficiently homogeneous alignment within the gel. The polymer homogeneity was evaluated by eye as well as the ${ }^{2} \mathrm{H}$ quadrupolar splitting of solvent signals in deuterium SSE experiments.

\subsection{NMR Experiments}

Measurements were performed at $25^{\circ} \mathrm{C}$ unless stated otherwise. Measurements were always performed on non-spinning samples. Spectra were recorded and processed with TopSpin 2.1 (Bruker Biospin, Rheinstetten, Germany). Further processing was done with MestreNova 10.0 (Mestrelab Research, Santiago de Compostela, Spain). Chemical shifts are reported in ppm relative to TMS $\left({ }^{1} \mathrm{H},{ }^{2} \mathrm{H}\right)$ and $\mathrm{LiCl}$ ( $\left.{ }^{7} \mathrm{Li}\right) . J$-couplings, RDCs and residual quadrupolar couplings are given in units of $\mathrm{Hz}$.

Special care was taken for the shimming of heterogeneous samples. Automated gradient shimming is usually not possible due to low signal-to-noise ratios or split deuterium signals. Likewise manual shimming on the lock signal proved impracticable due to low signal-to-noise ratios as well. Hence, shimming on the FID involved continuous acquisition of FIDs without saving but with parallel integration of the FID area. Homogeneously shimmed samples display larger integrals thereby enabling a quantitative observable to estimate and improve the field homogeneity.

\section{Imaging Experiment}

Imaging experiments were performed on a Bruker Avance III 400 spectrometer with a standard BBI probe with $z$ gradient. The pulse program echo_image.gau written in Bruker Avance syntax is given in the Appendix. After a $90^{\circ}$ hard pulse the magnetization develops under a gradient applied at $20 \%$ maximum strength for $10 \mathrm{~ms}$. This is followed by a $180^{\circ}$ pulse and signal acquisition under a gradient applied at $20 \%$ maximum strength. 


\section{SSE}

SSE NMR spectra were measured on a Bruker Avance III 400 spectrometer with a standard BBI probe with $z$ gradient. The pulse program $z g \_$slice.gau written in Bruker Avance syntax is given in the Appendix.

Slice-selective ${ }^{1} \mathrm{H}$ experiments were performed at $400.13 \mathrm{MHz}$ with a gradient strength of $25.6 \mathrm{G} / \mathrm{cm}$. Gauss-Cascade G4 shaped pules were used to excite a bandwidth of $\sim 11000 \mathrm{~Hz}$ which corresponds to $\sim 1 \mathrm{~mm}$ thick slices. Frequency offsets for these pulses ranged between $98209 \mathrm{~Hz}$ and $-98209 \mathrm{~Hz}$ with steps of $10912 \mathrm{~Hz}$. This resulted in 19 slices with $1 \mathrm{~mm}$ distance between each slice.

Slice-selective ${ }^{2} \mathrm{H}$ experiments were performed at $61.4 \mathrm{MHz}$ with a gradient strength of $51.1 \mathrm{G} / \mathrm{cm}$. G4 shaped pules were used to excite a bandwidth of $\sim 1700 \mathrm{~Hz}$ which corresponds to $\sim 0.5 \mathrm{~mm}$ thick slices. Frequency offsets for these pulses ranged between $23560 \mathrm{~Hz}$ and $-23560 \mathrm{~Hz}$ with steps of $3366 \mathrm{~Hz}$. This resulted in 15 slices with $1 \mathrm{~mm}$ distance between each slice.

Slice-selective ${ }^{7} \mathrm{Li}$ experiments were performed at $155.5 \mathrm{MHz}$ with a gradient strength of $51.1 \mathrm{G} / \mathrm{cm}$. G4 shaped pules were used to excite a bandwidth of $\sim 8500 \mathrm{~Hz}$ which corresponds to $\sim 1 \mathrm{~mm}$ thick slices. Frequency offsets for these pulses ranged between $76723 \mathrm{~Hz}$ and $-76723 \mathrm{~Hz}$ with steps of $8525 \mathrm{~Hz}$. This resulted in 19 slices with $1 \mathrm{~mm}$ distance between each slice.

The Bruker Topspin automation program poptau offers a simple method to vary different parameters, among them are frequency offsets. Additionally, results obtained by poptau can be saved as pseudo-2D spectra. Further customization of this AU can easily achieve a standardized and fast acquisition procedure.

\section{CPMG}

CPMG NMR spectra were measured on a Bruker Avance III 400 spectrometer with a standard BBI probe with $z$ gradient. The echo time before and after the $\pi$ pulse $(19.08 \mu \mathrm{s})$ was $2 \mathrm{~ms}$.

Transverse relaxation was monitored by echo trains of $1,2,5,10,20,40,80,150,250,450$ and 700 cycles (corresponding to a maximum of $1.82 \mathrm{~s}$ between $\pi /$ pulse and signal acquisition).

\section{DOSY}

DOSY measurements were performed on a Bruker Avance III 400 spectrometer equipped with a standard BBI probe with $z$ gradient. The pulse program dstebpgp3s was applied in the Bruker Topspin automation program xau dosy.

Herein, the pulsed gradient strength was incremented in 16 steps from $2 \%$ to $98 \%$. The diffusion time (50 ms) and the gradient (1.38 ms) were optimized beforehand to achieve a $\sim 100: 5$ signal attenuation between maximum and minimum gradient strength. 


\section{CLIP-HSQC}

CLIP-HSQC spectra were recorded using an INEPT delay of $1 /\left(4 \times{ }^{1} J_{\mathrm{CH}}\right)$ with ${ }^{1} J_{\mathrm{CH}}=145 \mathrm{~Hz}$. For the small molecules in the F1 dimension a total of 128 data points were sampled over a spectral width of $200 \mathrm{ppm}$. For the indanes a total of 256 data points were sampled over a spectral with of $200 \mathrm{ppm}$. In the F2 dimension 2048 data points were recorded with a spectral width of $15 \mathrm{ppm}$. The number of scans was 4 for the isotropic as well as anisotropic sample.

\section{NOESY}

NOESY spectra were recorded on a Bruker Avance III $500 \mathrm{MHz}$ instrument, using a relaxation delay of $2 \mathrm{~s}$. In the F1 dimension a total of 1024 data points were sampled over a spectral width of $8 \mathrm{ppm}$. In the F2 dimension 2048 data points were recorded with a spectral width of 8 ppm. Number of scans was 16 .

\section{HMBC}

Conventional HMBC spectra were recorded on a Bruker Avance III $500 \mathrm{MHz}$ instrument, using a relaxation delay of $2 \mathrm{~s}$. In the $\mathrm{F} 1$ dimension a total of 512 data points were sampled over a spectral width of $180 \mathrm{ppm}$. In the F2 dimension 4096 data points were recorded with a spectral width of $8 \mathrm{ppm}$, corresponding to an acquisition time of $0.5 \mathrm{~s}$. Number of scans was 8 . The $J$-couplings were determined from the peak multiplets along F2 with an accuracy of $\sim 1 \mathrm{~Hz}$. 


\subsection{Processing of NMR Data}

\section{Homogeneous Reference Samples}

Homogeneous reference samples for ${ }^{7} \mathrm{Li}$ were prepared by dissolving $\mathrm{LiClO}_{4}$ in toluene- $d_{8}$ to obtain three different solutions with known concentration $(0.49,0.58,0.73 \mathrm{~mol} / \mathrm{L})$ were prepared and measured via SSE. The signals were integrated and corrected according to the sensitivity profile. Homogeneous reference samples for ${ }^{1} \mathrm{H}$ were prepared by solving acetone in toluene- $d_{8}$ to obtain three different solutions with known concentration $(1.97,2.19,2.46 \mathrm{~mol} / \mathrm{L})$ were prepared and measured via SSE. The signals were integrated and corrected according to the sensitivity profile. Concentration $c$ can then be obtained from intensities $I$ through $c=\mathrm{m} \cdot I$ with $\mathrm{m}(\mathrm{H})=8.4 \cdot 10^{-4} \mathrm{~mol} / \mathrm{L}$ and $\mathrm{m}(\mathrm{Li})=1.71 \mathrm{~mol} / \mathrm{L}$. The obtained calibration curves are depicted in Figure 5-3.
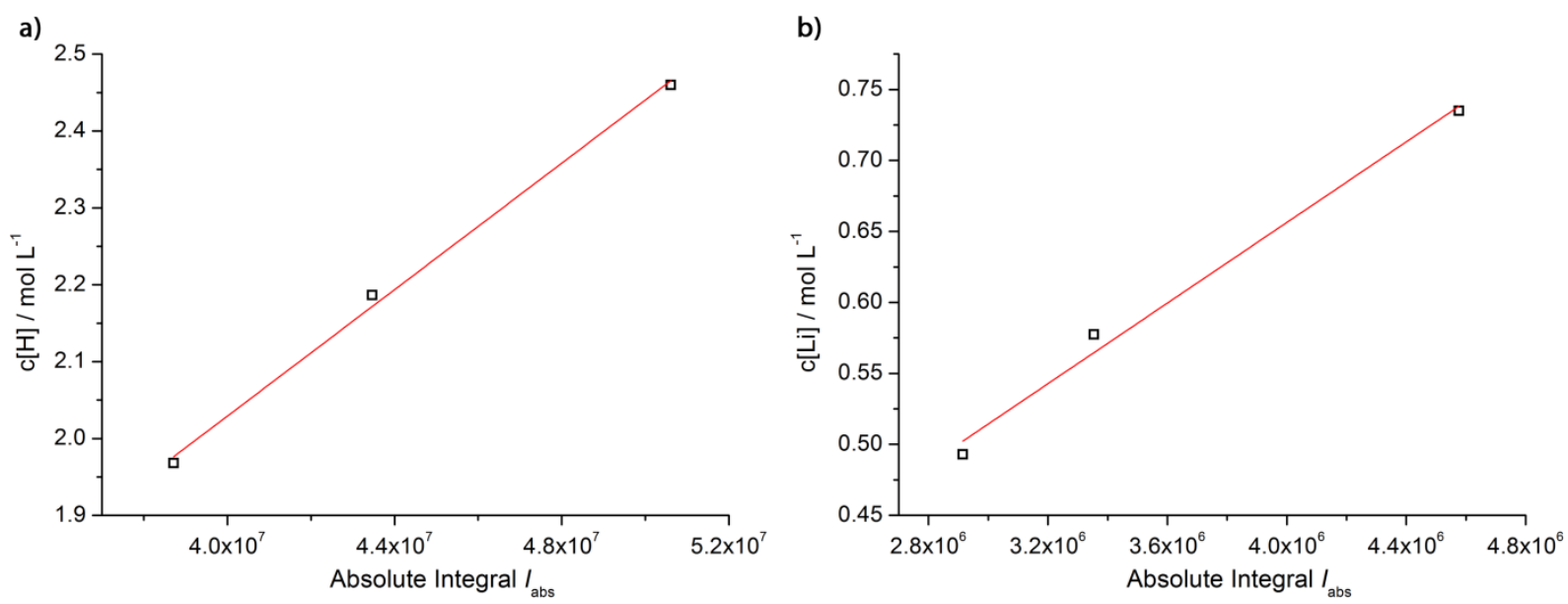

Figure 5-3: Calibration curves for the concentration determination of a) hydrogen-containing molecules and b) lithium-containing molecules. 


\section{RDC Data Analysis}

Isotropic measurements yielded ${ }^{1} J_{\mathrm{CH}}$ couplings by CLIP-HSQC ${ }^{[68]}$ Further CLIP-HSQC spectra of anisotropic samples were recorded to yield couplings ${ }^{1} T_{\mathrm{CH}}$ that are the sums of scalar and RDCs $J$ and $D$, respectively: ${ }^{1} T_{\mathrm{CH}}={ }^{1} J_{\mathrm{CH}}+{ }^{1} D_{\mathrm{CH}}$.

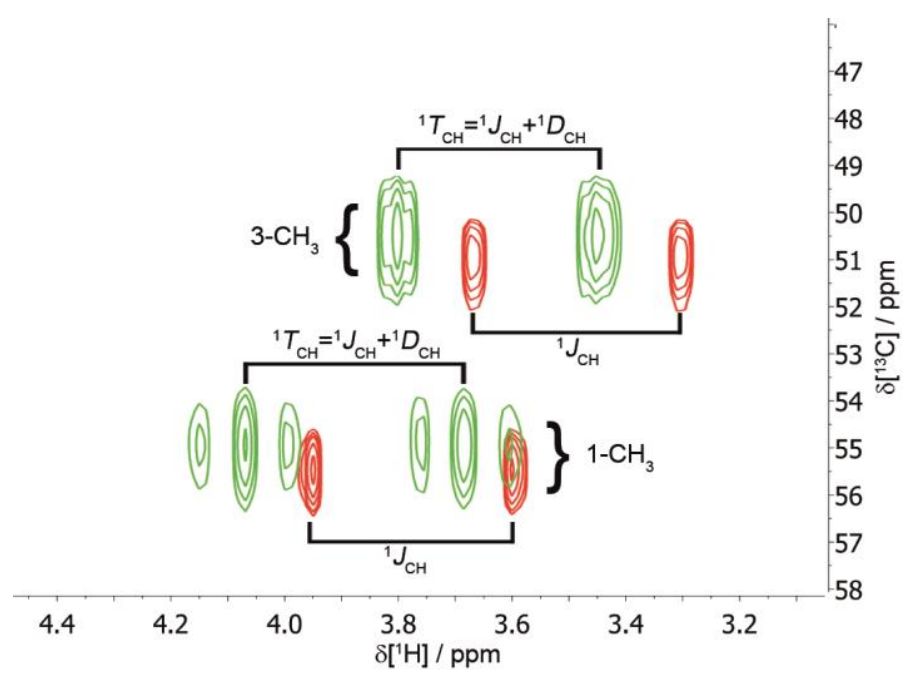

Figure 5-4: Excerpt of a CLIP-HSQC spectrum of $\beta$-aa-Me. Red: Isotropic ${ }^{1} J_{\mathrm{CH}}$ couplings. Green: Anisotropic ${ }^{1} T_{\mathrm{CH}}$ couplings. Note that for methyl groups an additional triplett appears through ${ }^{2} D_{\mathrm{HH}}$ couplings.

RDCs were saved as *.lab file. These contain the atom numbers of the $\mathrm{C}-\mathrm{H}$ pairs and their RDC values in $\mathrm{Hz}$. In the case of prochiral $\mathrm{H}$ atoms with unclear assignments a swapping of these RDCs can be achieved by the command "permutations". An exemplary *.lab file is included in the Appendix.

Within MSpin errors were computed with an estimated standard deviation of $0.5 \mathrm{~Hz}$. Furthermore methyl was treated as freely rotating and otherwise the defaults were applied. MSpin computes values for back-calculated RDCs and additionally gives a more detailed output concerning the alignment of the molecules. An exemplary output file is included in Appendix B Exemplary MSpin Input and Output. 


\section{DOSY Data Analysis}

Spectra as acquired via the xau dosy routine were processed with the help of the Topspin $T_{1} / T_{2}$ relaxation module (Figure 5-5).

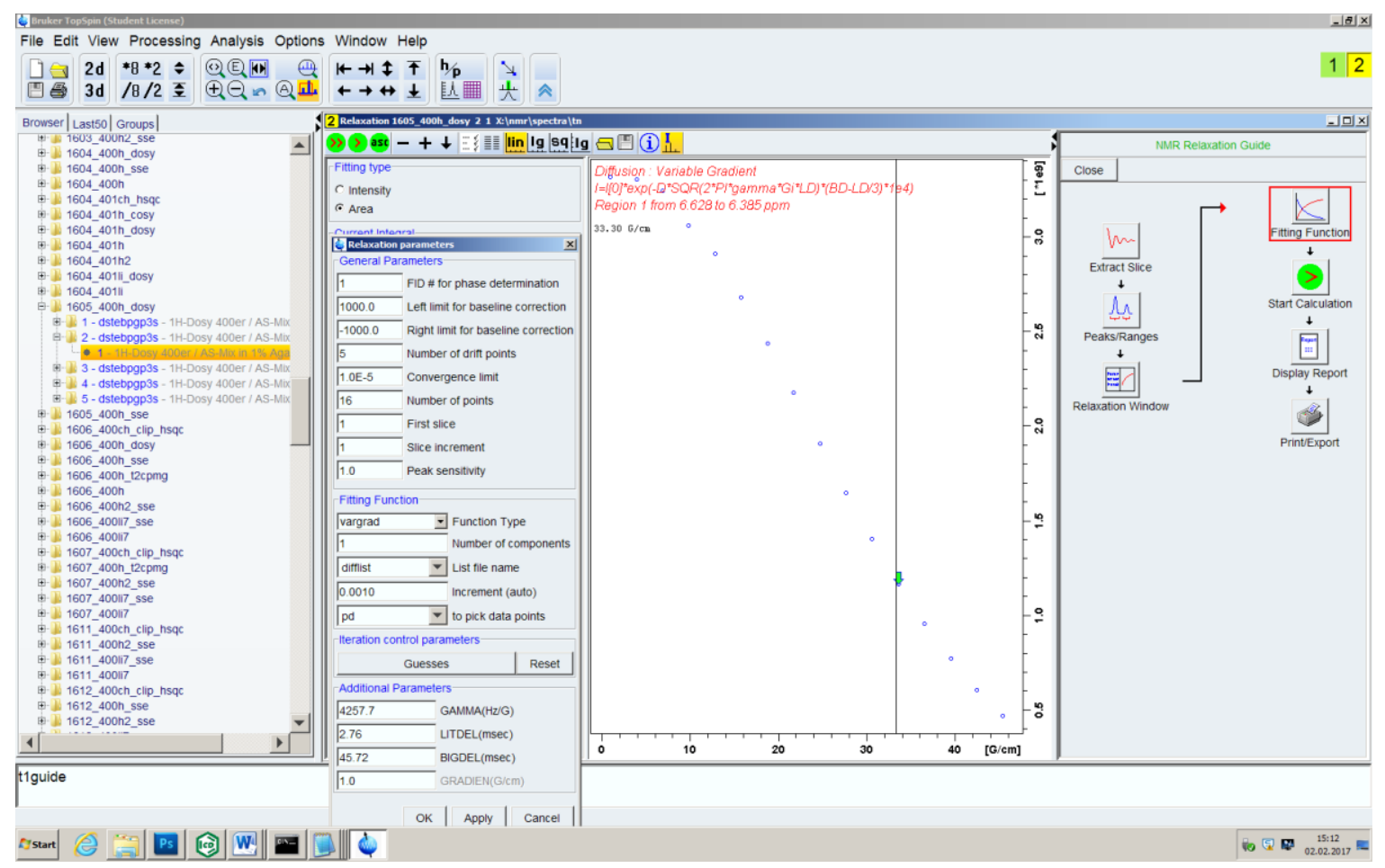

Figure 5-5: Screenshot of DOSY data processing for the extraction of diffusion coefficients from Gaussian fitting of the signal intensity or area decay.

This module reads either intensity or integral data for chosen signals and directly yields diffusion coefficients from Gaussian decay fitting. 


\subsection{Computational Techniques}

\section{Simulations and Analysis}

MD Simulations were performed using GROMACS 5.1.2 $2^{[139]}$ and the established OPLS-AA force field. ${ }^{[140,170]}$ Topologies were built according to GROMACS guidelines and with OPLS standard charges. Prior to the production run, systems were energy optimized. The corresponding production run file is given in Appendix B Exemplary MD Simulation Run File. MD production runs were performed in small cubic boxes with periodic boundary conditions (PS and PBA without DCM: $1.95 \mathrm{~nm}$, PS and PBA with DCM: $2.05 \mathrm{~nm}$, Figure 5-6).

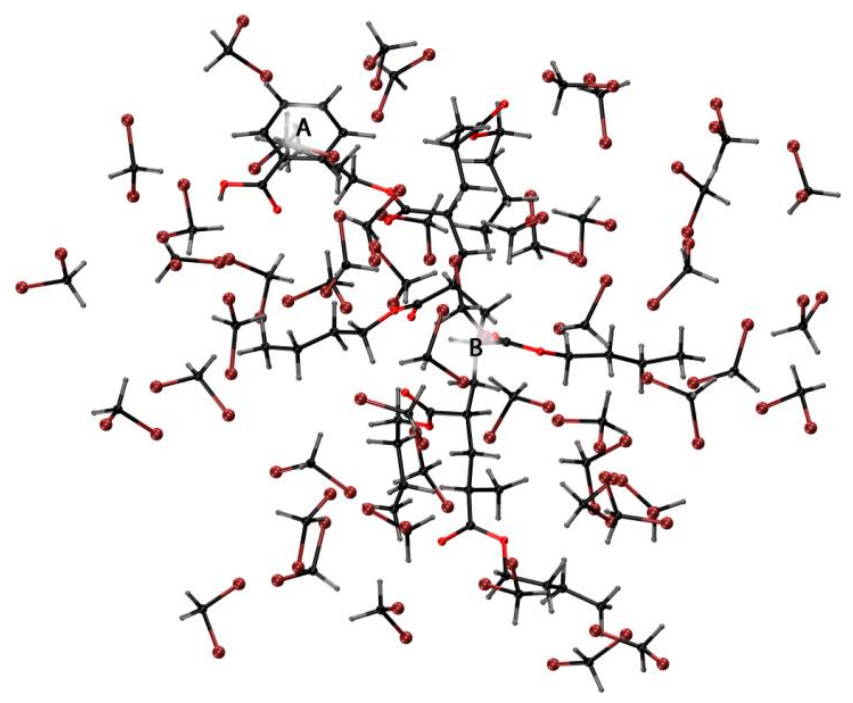

Figure 5-6: Screenshots of MD simulation box containing benzoic acid (A), PBA (B) and DCM molecules.

To mimic solvent effects in cases where no DCM molecules were added, Langevin dynamics were set on to add friction terms. During $400 \mathrm{~ns}$ production runs the Berendsen thermostat ${ }^{[141]}$ was set to a target temperature of $300 \mathrm{~K}$ with no preceding thermal equilibration. MD runs were analyzed with the GROMACS built-in utility gmx angle, the $\mathrm{VMD}^{[171]}$ molecular dynamics visualizing software and $M$. Brehm's Travis ${ }^{[138]}$ as a further tool to analyze trajectories from MD runs. In particular, Travis allowed the determination of spatial distribution functions as well as combined distribution functions.

\section{DFT Calculations of Optimized Structures and EFG Analysis}

DFT calculations were performed using ORCA 3.0.1. ${ }^{[154 a]}$ Exemplary input files are provided in Appendix B Exemplary ORCA input files geometry optimization and numerical frequencies. Optimized structures were evaluated as minima by numerically calculating vibrational frequencies which in turn must not include (large) imaginary wavenumbers. Electrostatic potentials were calculated through the CHELPG $^{[138]}$ routine as implemented in ORCA. EFG tensors were calculated using the fgrad keyword. Graphical representation of the EFG tensors by spherical polar plots was achieved by a Mathematica ${ }^{[172]}$ script provided publically ${ }^{[173]}$ by J. Autschbach (Figure 5-7). 


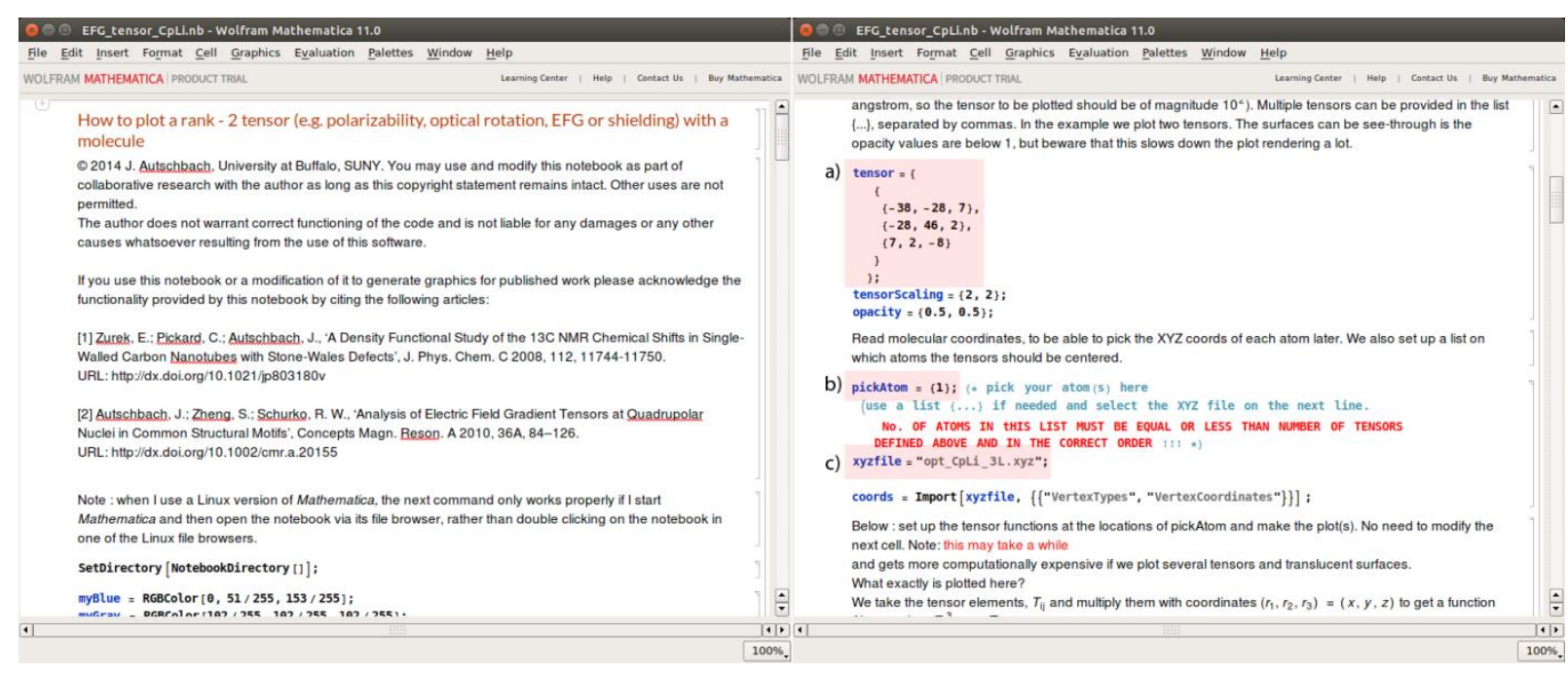

Figure 5-7: Screenshots of Mathematica script for tensor representation. Right window: a) Multiple of EFG as determined from ORCA. b) Number of atom with EFG in xyz file. c) xyz file correlated to ORCA EFG calculation. 


\section{APPENDIX}

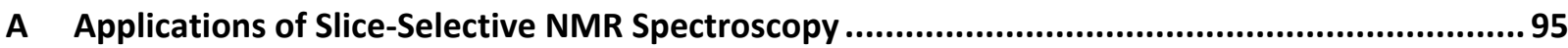

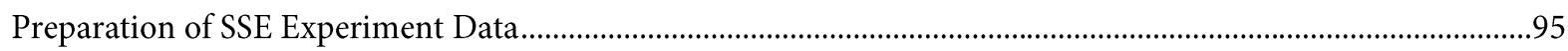

Imaging Pulse Sequence ................................................................................................................................95

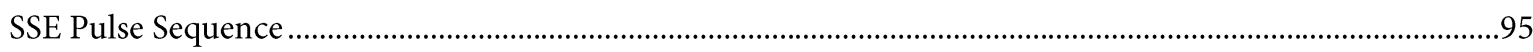

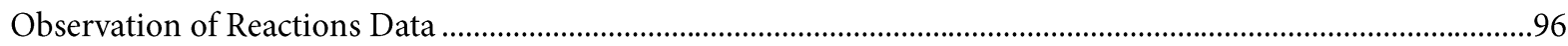

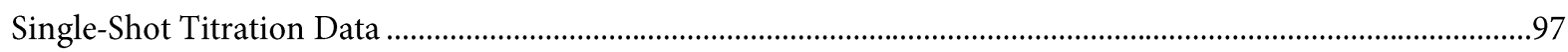

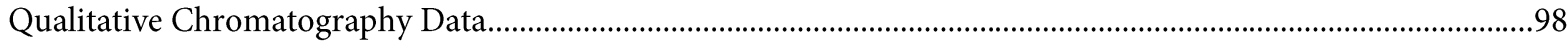

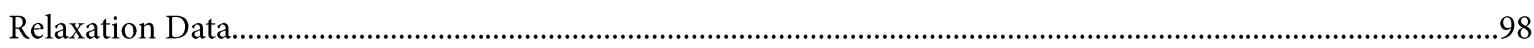

B Anisotropic NMR Spectroscopy in Polymeric Gels ....................................................................99

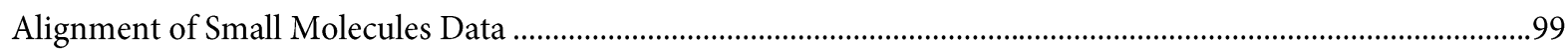

Least-Square Solution Script..............................................................................................................99

MD Energy Optimization Run File ......................................................................................................99

Exemplary MD Simulation Run File .....................................................................................................99

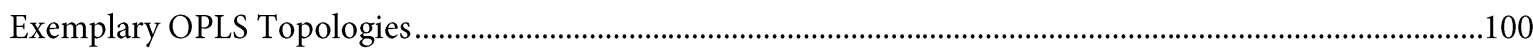

Structural Elucidation of Synthetic Indanes Data............................................................................................105

Exemplary ORCA input files geometry optimization and numerical frequencies ...............................................105

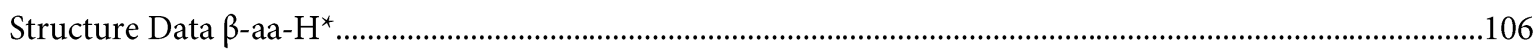

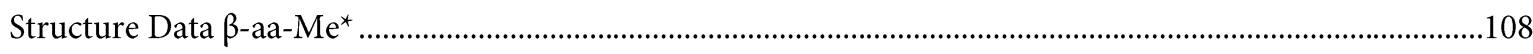

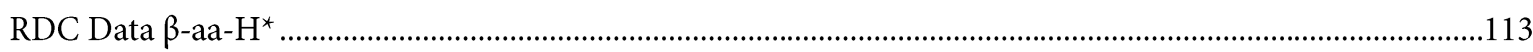

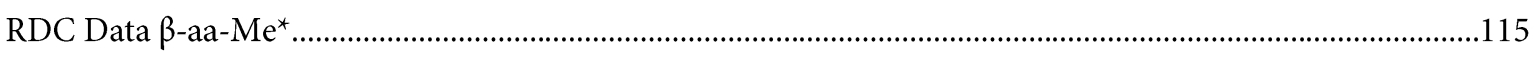

Exemplary MSpin Input and Output ....................................................................................................117

Structural Noise Script..........................................................................................................................117

Extended NMR Spectroscopy of Synthetic Indanes....................................................................................119

Quantitative Analysis of Residual Quadrupolar Couplings Data...........................................................................123

Exemplary ORCA input files EFG tensor calculation.......................................................................................123

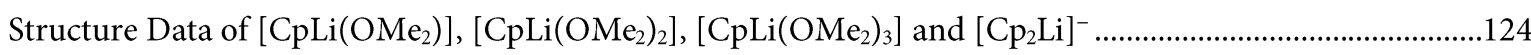




\section{A Applications of Slice-Selective NMR Spectroscopy}

\section{Preparation of SSE Experiment Data}

\section{Imaging Pulse Sequence}

Pulse program echo_image.gau in Bruker Avance syntax.

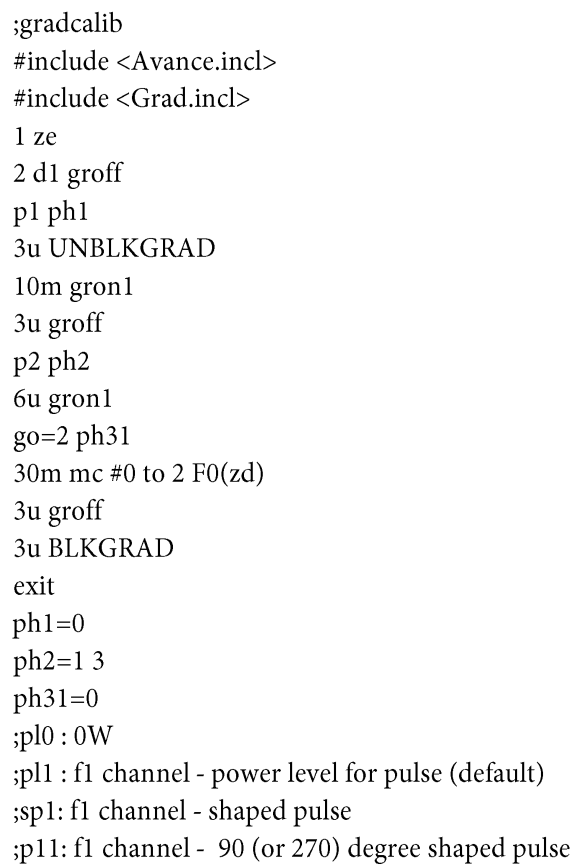

\section{SSE Pulse Sequence}

Pulse program zg_slice.gau in Bruker Avance syntax.

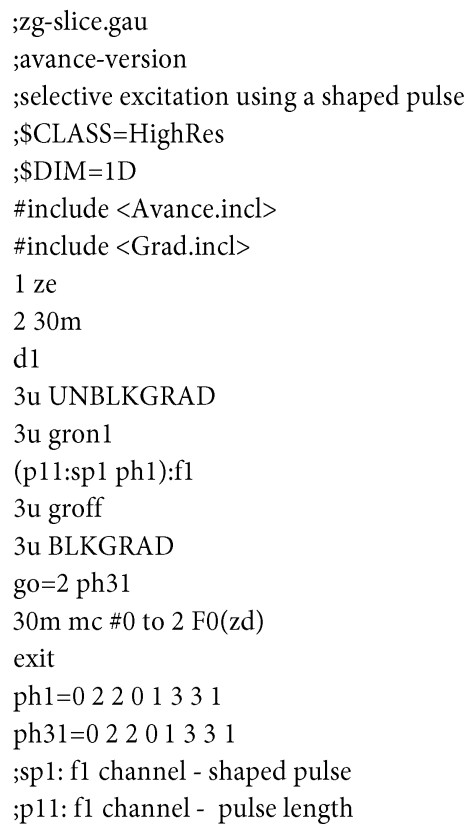


; 1 : relaxation delay; $1-5 * \mathrm{~T} 1$

;ns: $1^{*} \mathrm{n}$, total number of scans: NS * TD0

;choose p11 according to desired selectivity i.e. slice-width the flip-angle is determined by the amplitude

;choose frequency offsets according to desired slice distance for a given gradient strength

\section{Observation of Reactions Data}

Exemplary set of spectra:

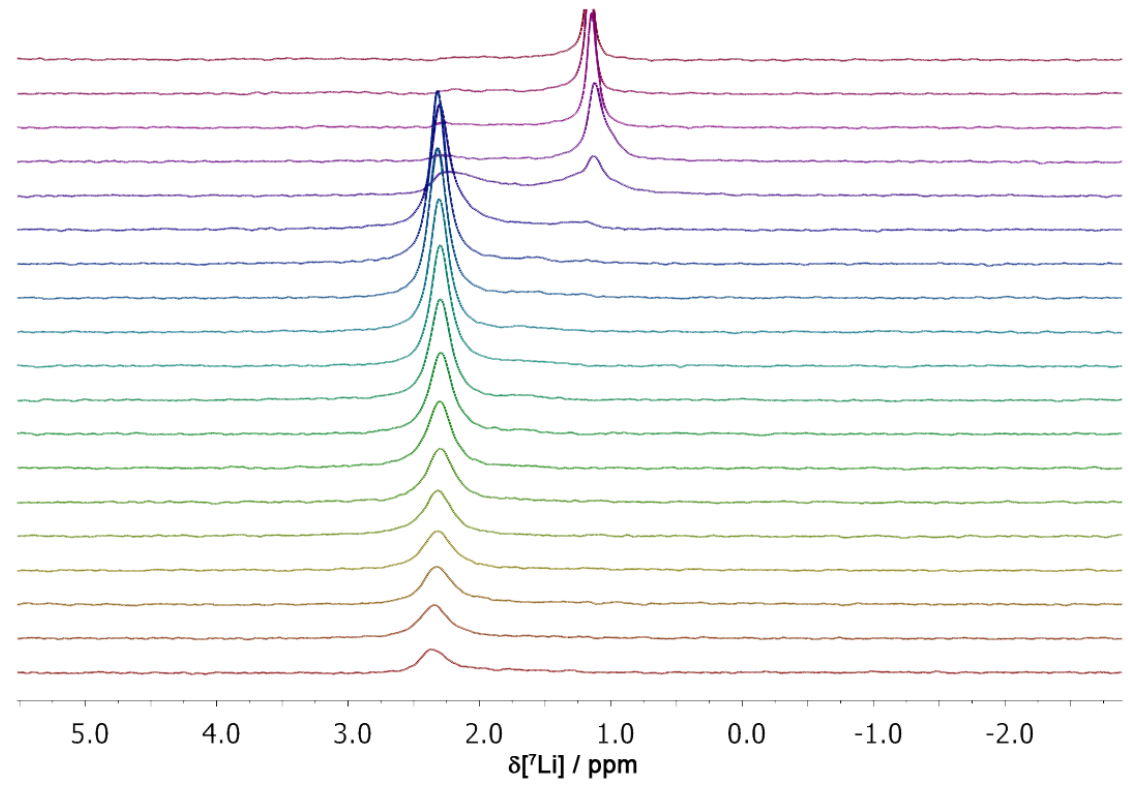

Figure A-1: SSE ${ }^{7} \mathrm{Li}$ spectra for the lithiation of PMDTA by $n \mathrm{BuLi}$. Spectra were taken approximately $3 \mathrm{~h}$ after addition of PMDTA.

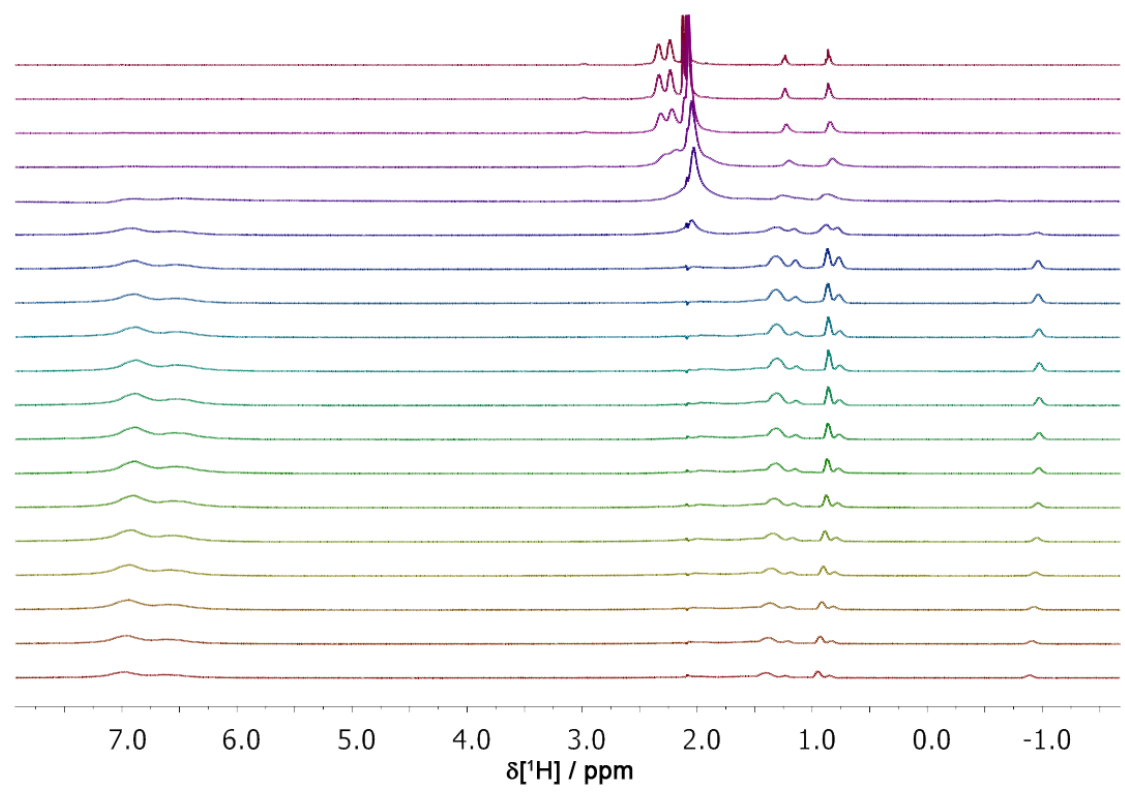

Figure A-2: SSE ${ }^{1} \mathrm{H}$ spectra for the lithiation of PMDTA by $n$ BuLi. Spectra were taken approximately $3 \mathrm{~h}$ after addition of PMDTA.

The full set of spectra is stored at the Stalke group server and can be obtained upon request. 


\section{Single-Shot Titration Data}

Exemplary set of spectra:

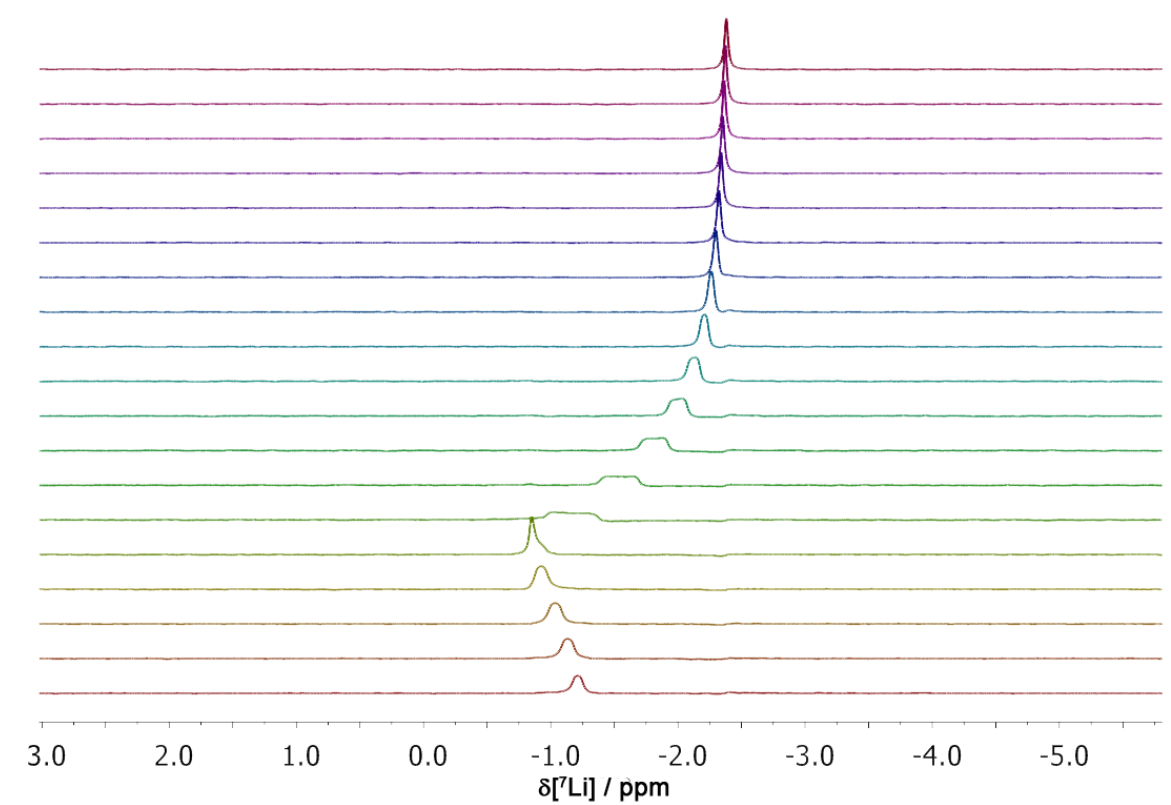

Figure A-3: SSE ${ }^{7} \mathrm{Li}$ spectra for the titration of $\mathrm{Li}^{+}$by 12 -crown-4. Spectra were taken approximately $3 \mathrm{~h}$ after preparation of the sample.

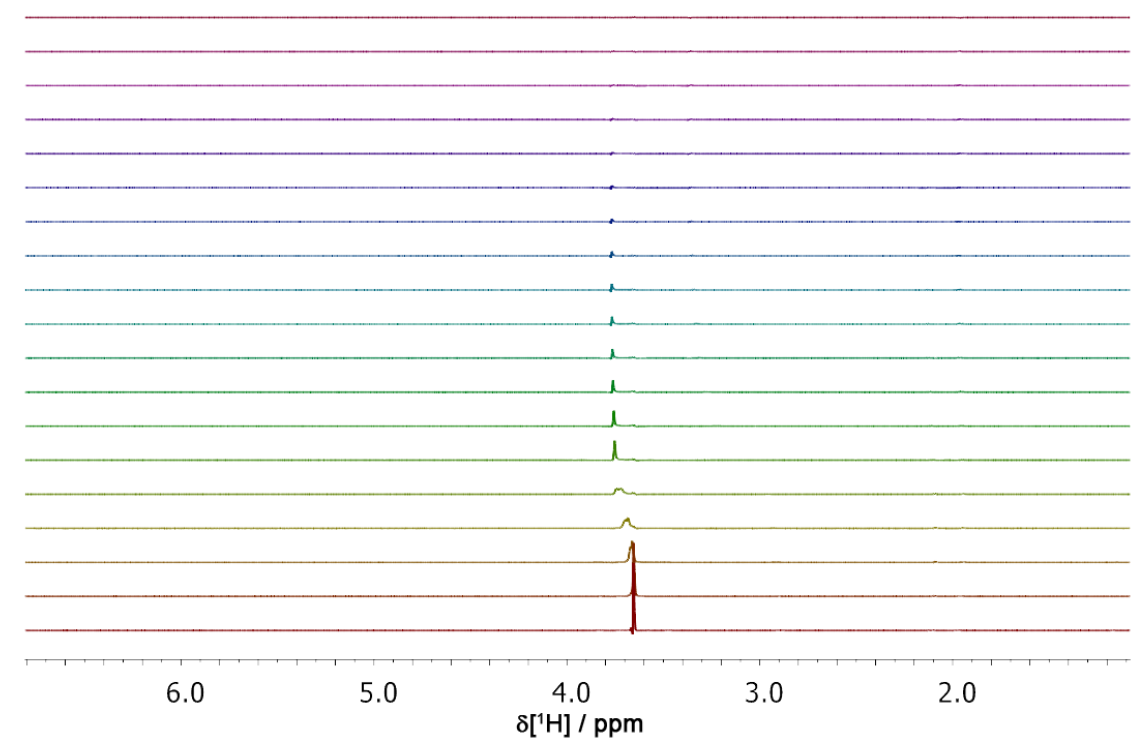

Figure A-4: SSE ${ }^{1} \mathrm{H}$ spectra for the titration of $\mathrm{Li}^{+}$by 12 -crown-4. Spectra were taken approximately $3 \mathrm{~h}$ after preparation of the sample.

The full set of spectra is stored at the Stalke group server and can be obtained upon request. 


\section{Qualitative Chromatography Data}

The full set of spectra is stored at the Stalke group server and can be obtained upon request. Exemplary output file from the $T_{1} / T_{2}$ relaxation module and analysis of the signal of L-His at the low field limit of the spectrum.

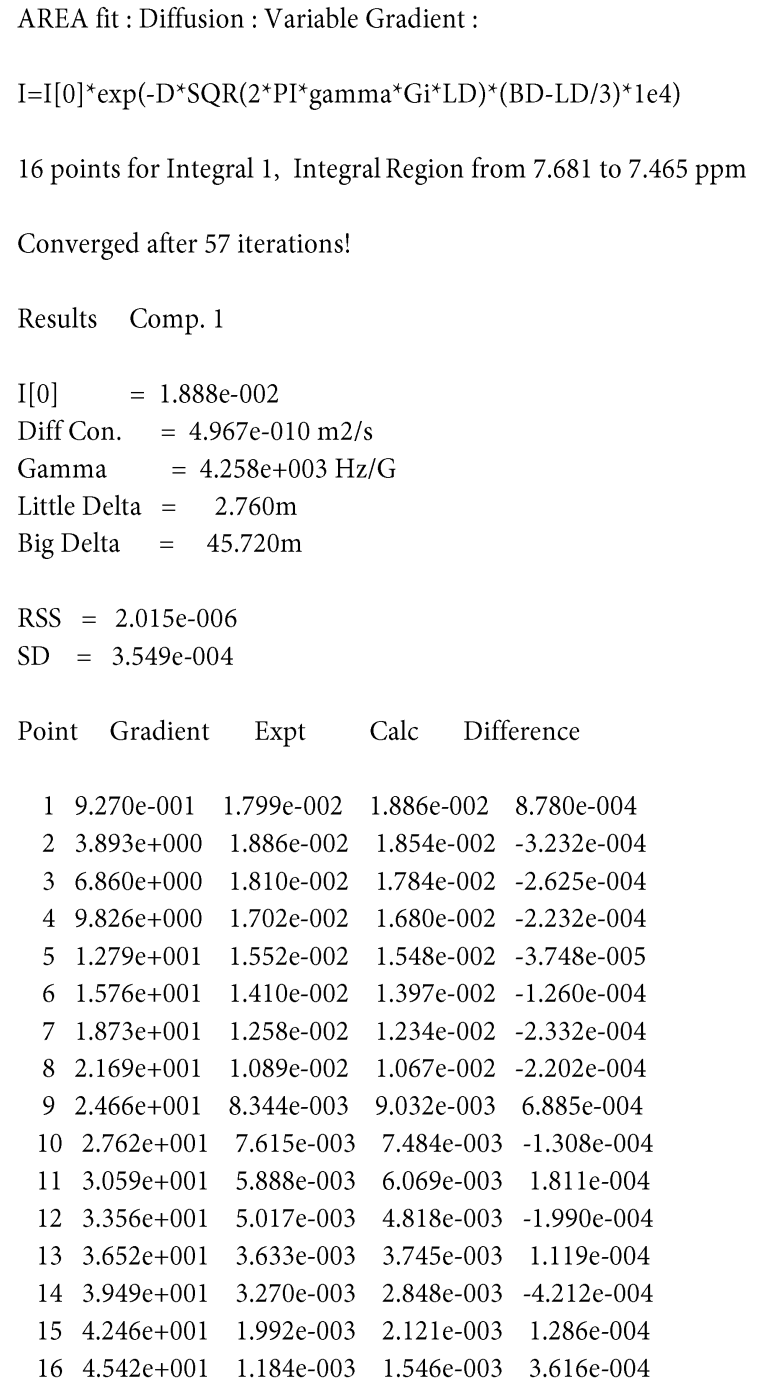

\section{Relaxation Data}

Table A-1: Relaxation data from CPMG experiments.

\begin{tabular}{cccc}
\hline$t / s$ & $2 w t \%$ Agarose & 3wt\% Agarose & 5wt\% Agarose \\
\hline 0.00804 & 1 & 1 & 1 \\
0.01206 & 0.9294 & 0.91439 & 0.9074 \\
0.02412 & 0.86402 & 0.82709 & 0.768 \\
0.04422 & 0.75438 & 0.69152 & 0.58333 \\
0.08442 & 0.56542 & 0.4788 & 0.33846 \\
0.16481 & 0.32781 & 0.23659 & 0.11695 \\
0.3256 & 0.1129 & 0.05845 & 0.01509 \\
0.60699 & 0.01809 & 0.00549 & $6.58 \mathrm{E}-04$ \\
1.00897 & 0.00148 & $2.40 \mathrm{E}-04$ & $5.94 \mathrm{E}-05$ \\
1.81293 & $2.00 \mathrm{E}-05$ & $9.45 \mathrm{E}-06$ & $2.37 \mathrm{E}-05$ \\
\hline
\end{tabular}




\section{B Anisotropic NMR Spectroscopy in Polymeric Gels}

\section{Alignment of Small Molecules Data}

\section{Least-Square Solution Script}

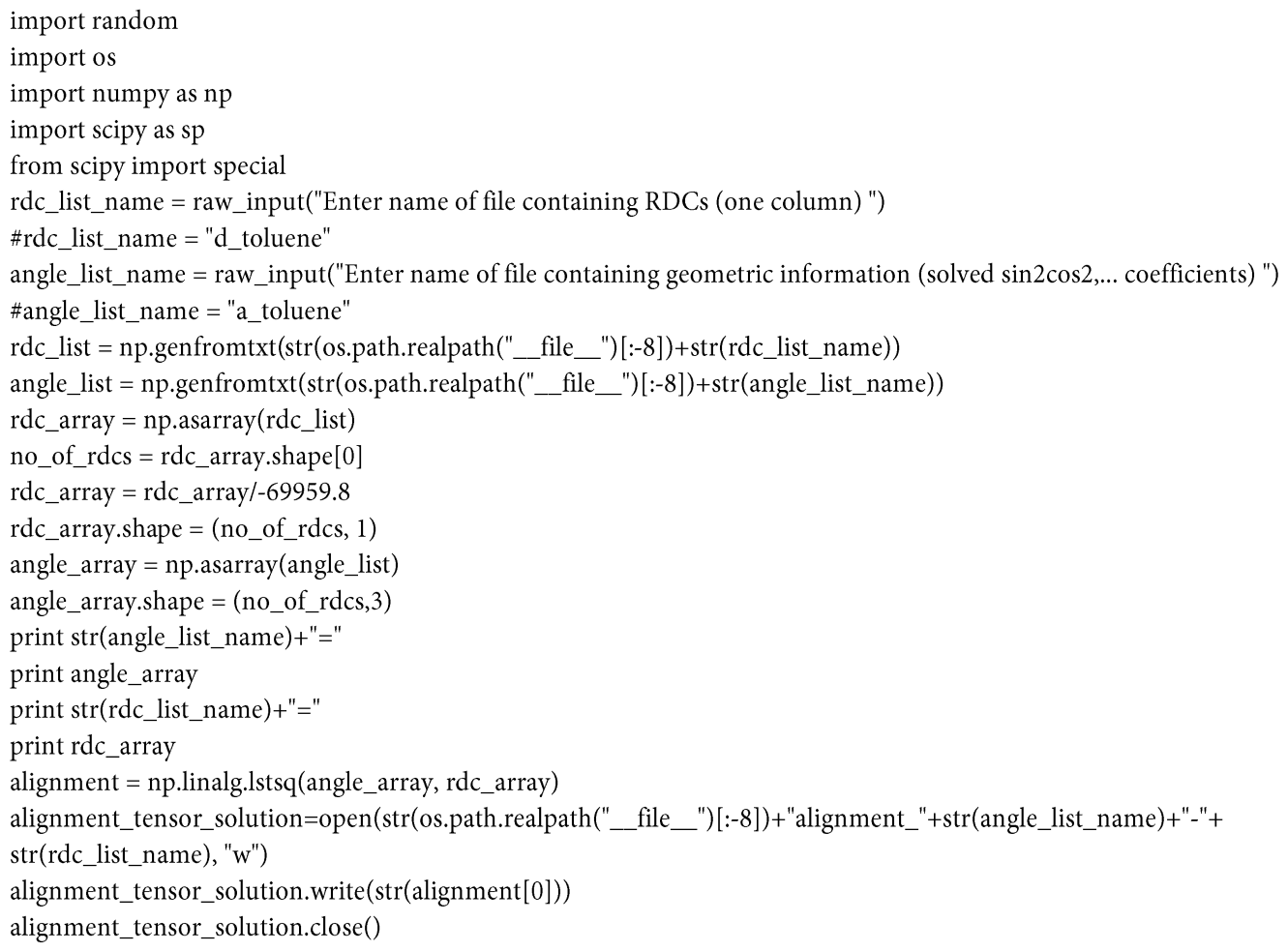

\section{Energy Optimization Run File}

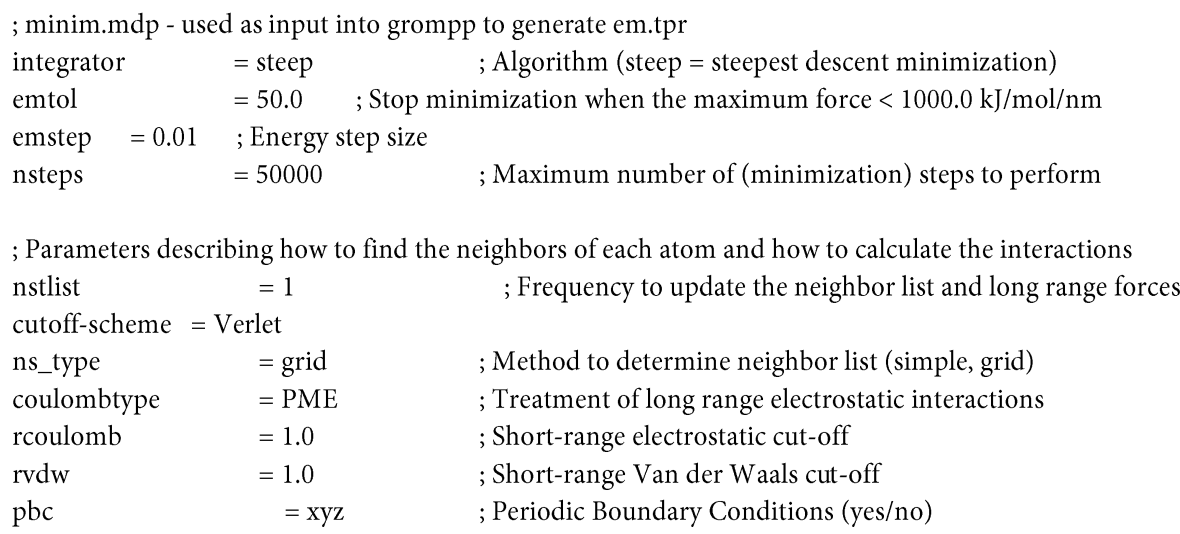

\section{Exemplary MD Simulation Run File}

Adapted MD simulation script with comments:

; run.mdp - used as input into grompp to generate run.tpr

; Run parameters

integrator $\quad$ md leap-frog integrator 


\begin{tabular}{|c|c|c|}
\hline nsteps & $=200000000$ & $; 2 * 200000000=400000 \mathrm{ps}(400 \mathrm{~ns})$ \\
\hline $\mathrm{dt}$ & $=0.002$ & $; 2$ fs \\
\hline \multicolumn{3}{|l|}{; Output control } \\
\hline nstxout & $=5000$ & ; save coordinates every $10.0 \mathrm{ps}$ \\
\hline nstvout & $=5000$ & ; save velocities every $10.0 \mathrm{ps}$ \\
\hline nstenergy & $=5000$ & ; save energies every $10.0 \mathrm{ps}$ \\
\hline nstlog & $=5000$ & ; update log file every $10.0 \mathrm{ps}$ \\
\hline \multicolumn{3}{|c|}{ nstxout-compressed $=5000 \quad ;$ save compressed coordinates every $10.0 \mathrm{ps}$} \\
\hline \multicolumn{3}{|c|}{ compressed-x-grps $=$ System $\quad ;$ replaces xtc-grps } \\
\hline \multicolumn{3}{|c|}{; Bond parameters } \\
\hline continuation & $=$ yes & ; Restarting after NPT \\
\hline \multicolumn{3}{|c|}{ constraint_algorithm $=$ lincs $\quad ;$ holonomic constraints } \\
\hline \multicolumn{3}{|c|}{ constraints $\quad=$ all-bonds $\quad$; all bonds (even heavy atom- $\mathrm{H}$ bonds) constrained } \\
\hline \multicolumn{3}{|c|}{$=1 \quad ;$ accuracy of LINCS } \\
\hline \multicolumn{3}{|c|}{ lincs_order } \\
\hline \multicolumn{3}{|c|}{$\begin{array}{l}\text {; Neighborsearching } \\
\text { cutoff-scheme = Verlet }\end{array}$} \\
\hline ns_type & = grid & ; search neighboring grid cells \\
\hline nstlist & $=10$ & largely irrelevant with Verlet scheme \\
\hline rcoulomb & $=1.0$ & ; short-range electrostatic cutoff (in nm) \\
\hline $\mathrm{rvdw}$ & $=1.0$ & ; short-range van der Waals cutoff (in $\mathrm{nm}$ ) \\
\hline \multicolumn{3}{|l|}{; Electrostatics } \\
\hline coulombtype & $=\mathrm{PME}$ & ; Particle Mesh Ewald for long-range electrostatics \\
\hline pme_order & $=4$ & ; cubic interpolation \\
\hline fourierspacing & $=0.16$ & ; grid spacing for FFT \\
\hline \multicolumn{3}{|c|}{; Temperature coupling is on } \\
\hline tcoupl & $=\mathrm{v}$-rescale & ; modified Berendsen thermostat \\
\hline tc_grps & \multicolumn{2}{|c|}{$=$ System $;$ One group } \\
\hline tau_t & $=0.1$ & ; time constant, in ps \\
\hline ref_t & $=300$ & ; reference temperature, one for each group, in $\mathrm{K}$ \\
\hline \multicolumn{3}{|c|}{; Pressure coupling is on } \\
\hline \multirow{2}{*}{\multicolumn{3}{|c|}{$\begin{array}{l}\text { pcoupl = no ; no pressure coupling } \\
\text {; Periodic boundary conditions }\end{array}$}} \\
\hline & & \\
\hline & $=x y z$ & ; 3-D PBC \\
\hline \multicolumn{3}{|c|}{; Dispersion correction } \\
\hline DispCorr & $=$ EnerPres & ; account for cut-off vdW scheme \\
\hline \multicolumn{3}{|c|}{; Velocity generation } \\
\hline gen_vel & $=$ no & ; Velocity generation is off \\
\hline
\end{tabular}

\section{Exemplary OPLS Topologies}

\section{Polybutylacrylate Topology}

See reference [132] for original simulation and topology on PS. Note that below only shortened topologies of PBA, toluene and benzoic acid are shown. These do not include entries for bonds, pairs and proper dihedrals. The full topologies are stored at the Stalke group server and can be obtained upon request.

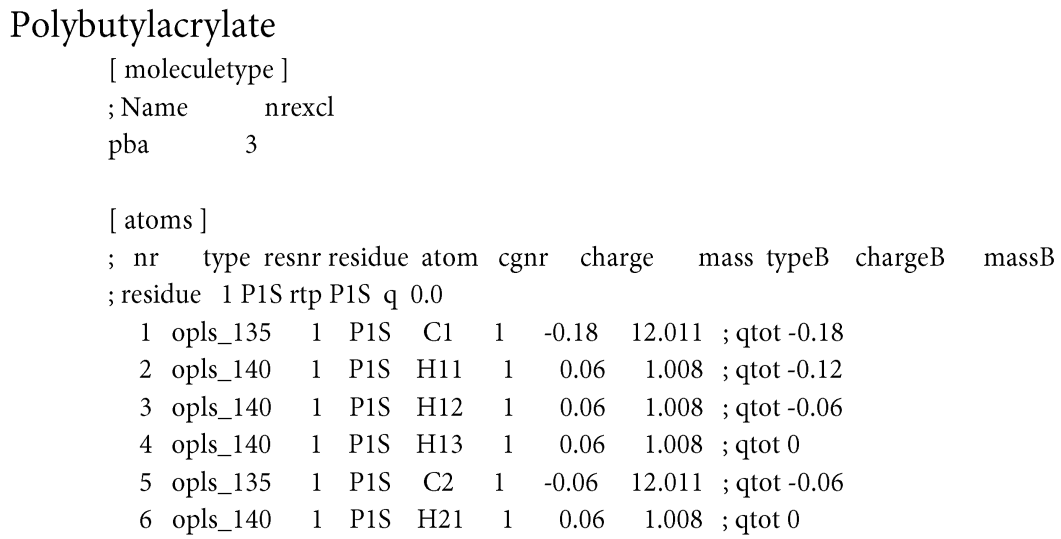




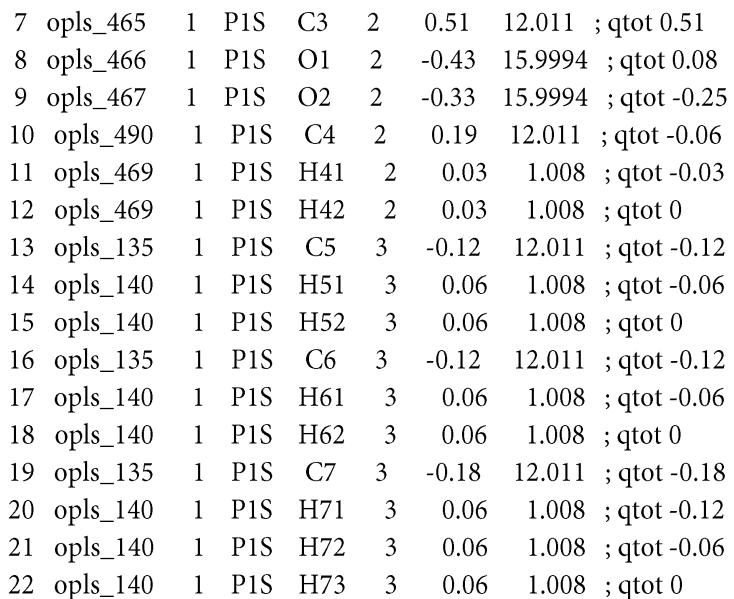

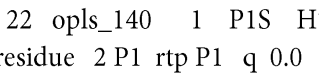

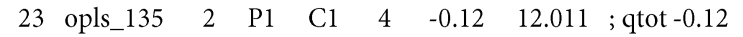

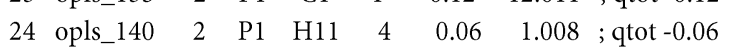

25 opls_140 2 P1 $\mathrm{H} 12 \quad 4 \quad 0.06 \quad 1.008$; qtot 0

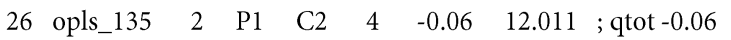

27 opls_140 2 P1 $\quad$ H21 $4 \quad 0.06 \quad 1.008$; qtot 0

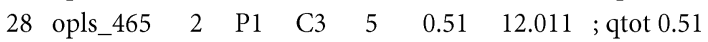

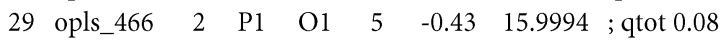

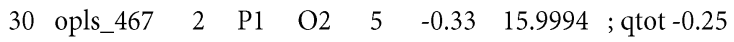

31 opls_490 22 P1 $\quad$ C4 $5 \quad 5 \quad 0.19 \quad 12.011$; qtot -0.06

32 opls_469 2 P1 $\mathrm{H} 41 \quad 5 \quad 0.03 \quad 1.008 ;$ qtot -0.03

33 opls_469 2 P1 $\mathrm{H} 42 \quad 5 \quad 0.03 \quad 1.008$; qtot 0

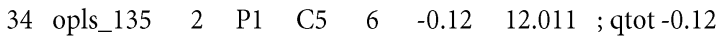

35 opls_140 2 2 1 P1 $\mathrm{H} 51 \quad 6 \quad 0.06 \quad 1.008 ;$ qtot -0.06

36 opls_140 2 P1 $\mathrm{H} 52 \quad 6 \quad 0.06 \quad 1.008$; qtot 0

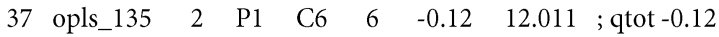

38 opls_140 2 P1 $\mathrm{H} 61 \quad 6 \quad 0.06 \quad 1.008 ;$ q qtot -0.06

39 opls_140 2 P1 $\mathrm{H6} 6{ }^{2} \quad 6 \quad 0.06 \quad 1.008$; qtot 0

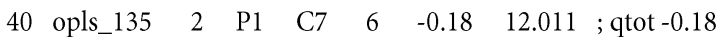

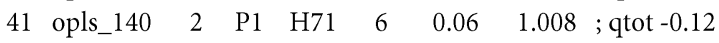

42 opls_140 22 P1 $\quad$ H72 $6 \quad 6 \quad 0.06 \quad 1.008$; qtot -0.06

43 opls_140 2 P1 $\mathrm{H73} \quad 6 \quad 0.06 \quad 1.008$; qtot 0

; residue $3 \mathrm{P} 1 \mathrm{rtp} P 1 \quad \mathrm{q} 0.0$

$\begin{array}{llllllll}44 & \text { opls_135 } & 3 & \text { P1 } & \text { Cl } & 7 & -0.12 & 12.011 ; \text { q tot }-0.12\end{array}$

45 opls_140 3 P1 $\mathrm{H} 11 \quad 7 \quad 0.06 \quad 1.008 ;$ qtot -0.06

46 opls_140 3 P1 $\mathrm{H} 12 \quad 7 \quad 0.06 \quad 1.008$; qtot 0

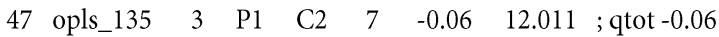

48 opls_140 3 P1 $321 \quad 7 \quad 0.06 \quad 1.008$; qtot 0

49 opls_465 $3 \begin{array}{llllll}3 & \text { P1 } & \text { C3 } & 8 & 0.51 & 12.011 ; q \text { qtot } 0.51\end{array}$

$\begin{array}{llllllll}50 & \text { opls_466 } & 3 & \text { P1 } & \text { O1 } & 8 & -0.43 & 15.9994 ; \text { q } q \text { tot } 0.08\end{array}$

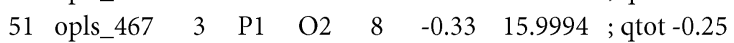

52 opls_490 33 P1 $\quad$ C4 $\quad 8 \quad 0.19 \quad 12.011$; qtot -0.06

53 opls_469 3 P1 $\quad$ H41 $8 \quad 0.03 \quad 1.008$; qtot -0.03

54 opls_469 3 P1 $\mathrm{H} 42 \quad 8 \quad 0.03 \quad 1.008$; qtot 0

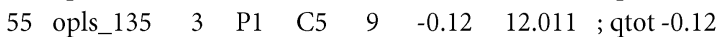

56 opls_140 3 P1 $\quad$ H51 $90.06 \quad 1.008$; qtot -0.06

57 opls_140 3 P1 $\quad$ H52 $900.06 \quad 1.008$; qtot 0

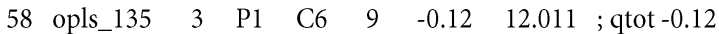

59 opls_140 3 P1 $\mathrm{H} 61 \quad 9 \quad 0.06 \quad 1.008$; qtot -0.06

60 opls_140 3 P1 $\quad$ H62 $900.06 \quad 1.008$; qtot 0

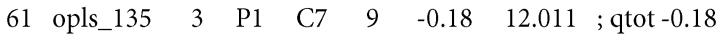

62 opls_140 3 P1 $\quad$ H71 $90.06 \quad 1.008 ;$ qtot -0.12

63 opls_140 3 P1 $\mathrm{H72} \quad 9 \quad 0.06 \quad 1.008$; qtot -0.06

64 opls_140 3 P1 $\quad$ H73 $90.06 \quad 1.008 ;$ qtot 0

; residue 4 P1 rtp P1 q 0.0

65 opls_135 44 P1 $\quad$ C1 $10 \quad-0.12 \quad 12.011$; qtot -0.12

66 opls_140 4 P1 H11 $10 \quad 0.06 \quad 1.008$; qtot -0.06

67 opls_140 4 P1 $\mathrm{H12} \quad 10 \quad 0.06 \quad 1.008$; qtot 0

68 opls_135 4 P1 $\quad$ C2 $10 \quad-0.06 \quad 12.011$; qtot -0.06

69 opls_140 $\quad 4 \quad$ P1 $\quad$ H21 $10 \quad 0.06 \quad 1.008$; qtot 0 


\begin{tabular}{|c|c|c|c|c|c|c|c|}
\hline 70 & opls_465 & 4 & $\mathrm{P} 1$ & $\mathrm{C} 3$ & 11 & 0.51 & $12.011 ;$ qtot 0.51 \\
\hline 71 & opls_466 & 4 & P1 & O1 & 11 & -0.43 & 15.9994 ; qtot 0.08 \\
\hline 72 & opls_467 & 4 & P1 & $\mathrm{O} 2$ & 11 & -0.33 & $15.9994 ; \mathrm{qt}$ \\
\hline 73 & opls_490 & 4 & P1 & $\mathrm{C} 4$ & 11 & 0.19 & $12.011 ;$ qto \\
\hline 74 & opls_469 & 4 & P1 & $\mathrm{H} 41$ & 11 & 0.03 & $1.008 ;$ qtot -0.03 \\
\hline 75 & opls_469 & 4 & P1 & $\mathrm{H} 42$ & 11 & 0.03 & $1.008 ;$ qtot 0 \\
\hline 76 & opls_135 & 4 & P1 & C5 & 12 & -0.12 & $12.011 ;$ qtot -0.12 \\
\hline 77 & opls_140 & 4 & P1 & $\mathrm{H} 51$ & 12 & 0.06 & $1.008 ; \mathrm{q}$ \\
\hline 78 & opls_140 & 4 & P1 & H52 & 12 & 0.06 & $1.008 ;$ qtot 0 \\
\hline 79 & opls_135 & 4 & P1 & $\mathrm{C} 6$ & 12 & -0.12 & $12.011 ;$ qtot -0.12 \\
\hline 80 & opls_140 & 4 & P1 & H61 & 12 & 0.06 & $1.008 ;$ qto \\
\hline 81 & opls_140 & 4 & P1 & H62 & 12 & 0.06 & $1.008 ; \mathrm{q}$ \\
\hline 82 & opls_135 & 4 & P1 & C7 & 12 & -0.18 & $12.011 ; \mathrm{qt}$ \\
\hline 83 & opls & 4 & P1 & H71 & 12 & 0.06 & $1.008 ;$ qto \\
\hline 84 & opls_140 & 4 & P1 & H72 & 12 & 0.06 & $1.008 ; \mathrm{qtc}$ \\
\hline 8 & opls & 4 & P1 & $\mathrm{H} 73$ & 12 & 0.06 & $1.008 ; \mathrm{q}$ \\
\hline \multicolumn{8}{|c|}{; residue 5 P1 rtp P1 q 0.0} \\
\hline 86 & opls_135 & 5 & P1 & $\mathrm{C} 1$ & 13 & -0.12 & $12.011 ;$ qtot -0.12 \\
\hline & opls & 5 & P1 & H11 & 13 & 0.06 & $1.008 ; \mathrm{qt}$ \\
\hline & opls & 5 & P1 & $\mathrm{H} 12$ & 13 & 0.06 & $1.008 ; \mathrm{qtc}$ \\
\hline 89 & opls & 5 & P1 & $\mathrm{C} 2$ & 13 & -0.06 & $12.011 ;$ qtot -0.06 \\
\hline 90 & opls & 5 & P1 & $\mathrm{H} 21$ & 13 & 0.06 & $1.008 ; \mathrm{q}$ \\
\hline 9 & & 5 & P1 & $\mathrm{C} 3$ & 14 & 0.51 & $12.011 ; \mathrm{q}$ \\
\hline 9 & & 5 & P1 & O1 & 14 & -0.43 & $15.9994 ;$ \\
\hline 9 & opls_467 & 5 & P1 & $\mathrm{O} 2$ & 14 & -0.33 & $15.9994 ;$ qtc \\
\hline 94 & opls & 5 & P1 & $\mathrm{C} 4$ & 14 & 0.19 & $12.011 ;$ qto \\
\hline 95 & opls & 5 & P1 & $\mathrm{H} 41$ & 14 & 0.03 & $1.008 ; \mathrm{q}$ \\
\hline 96 & opls_469 & 5 & P1 & $\mathrm{H} 42$ & 14 & 0.03 & $1.008 ; \mathrm{qt}$ \\
\hline 97 & opl & 5 & P1 & C5 & 15 & -0.12 & $12.011 ; \mathrm{qtc}$ \\
\hline 9 & opls & 5 & P1 & $\mathrm{H} 51$ & 15 & 0.06 & $1.008 ; \mathrm{qt}$ \\
\hline 99 & opls_140 & 5 & P1 & $\mathrm{H} 52$ & 15 & 0.06 & $1.008 ;$ qtot \\
\hline 100 & 135 & 5 & P1 & C6 & 15 & -0.12 & $12.011 ; \mathrm{c}$ \\
\hline 101 & 140 & 5 & P1 & H61 & 15 & 0.06 & $1.008 ; \mathrm{q}$ \\
\hline 102 & 140 & 5 & P1 & H62 & 15 & 0.06 & $1.008 ; \mathrm{qtc}$ \\
\hline 103 & opls_135 & 5 & P1 & $\mathrm{C} 7$ & 15 & -0.18 & $12.011 ;$ qtot -0.18 \\
\hline 104 & 140 & 5 & P1 & $\mathrm{H} 71$ & 15 & 0.06 & 1.008 ; qto \\
\hline 105 & opls_140 & 5 & P1 & $\mathrm{H} 72$ & 15 & 0.06 & $1.008 ;$ qtot -0.06 \\
\hline 106 & opls_140 & 5 & P1 & H73 & 15 & 0.06 & 1.008 ; qtot 0 \\
\hline \multicolumn{8}{|c|}{; residue 6 P1E rtp P1E q 0.0} \\
\hline 107 & opls_135 & 6 & P1E & $\mathrm{C} 1$ & 16 & -0.12 & $12.011 ;$ qtot -0.12 \\
\hline 108 & opls_140 & 6 & P1E & H11 & 16 & 0.06 & $1.008 ;$ qtot -0.06 \\
\hline 109 & opls_140 & 6 & P1E & H12 & 16 & 0.06 & 1.008 ; qtot \\
\hline 110 & opls_135 & 6 & P1E & $\mathrm{C} 2$ & 16 & -0.06 & $12.011 ;$ qtot -0.0 \\
\hline 111 & opl & 6 & P1E & $\mathrm{H} 21$ & 16 & 0.06 & 1.008 ; qto \\
\hline 112 & opl & 6 & P1E & C3 & 17 & 0.51 & $12.011 ;$ qtot 0.51 \\
\hline 113 & opls_466 & 6 & P1E & O1 & 17 & -0.43 & 15.9994 ; qtot 0.08 \\
\hline 114 & opls_467 & 6 & P1E & $\mathrm{O} 2$ & 17 & -0.33 & $15.9994 ;$ qtot -0.2 \\
\hline 115 & opls & 6 & P1E & $\mathrm{C} 4$ & 17 & 0.19 & $12.011 ;$ qtot -0.06 \\
\hline 116 & opl & 6 & P1E & $\mathrm{H} 4 \mathrm{I}$ & 17 & 0.03 & $1.008 ; \mathrm{q}$ \\
\hline 117 & opls & 6 & P1E & $\mathrm{H} 42$ & 17 & 0.03 & 1.008 ; qto \\
\hline 118 & opls_135 & 6 & P1E & $\mathrm{C} 5$ & 18 & -0.12 & $12.011 ;$ qtot -0.12 \\
\hline 119 & opls_140 & 6 & P1E & $\mathrm{H} 51$ & 18 & 0.06 & $1.008 ;$ qtot -0.06 \\
\hline 120 & opls_140 & 6 & P1E & H52 & 18 & 0.06 & $1.008 ;$ qtot 0 \\
\hline 121 & opls_135 & 6 & P1E & $\mathrm{C} 6$ & 18 & -0.12 & $12.011 ;$ qtot -0.1 \\
\hline 122 & opls_140 & 6 & P1E & H61 & 18 & 0.06 & $1.008 ;$ qtot -0.0 \\
\hline 123 & opls_140 & 6 & P1E & H62 & 18 & 0.06 & 1.008 ; qtot 0 \\
\hline 124 & opls_135 & 6 & P1E & $\mathrm{C} 7$ & 18 & -0.18 & $12.011 ;$ qtot -0.18 \\
\hline 125 & opls_140 & 6 & P1E & H71 & 18 & 0.06 & $1.008 ;$ qtot -0.12 \\
\hline 126 & -140 & 6 & P1E & H72 & 18 & 0.0 & $1.008 ;$ qtot -0.06 \\
\hline 127 & opls_140 & 6 & P1E & H73 & 18 & 0.06 & $1.008 ;$ qtot 0 \\
\hline 128 & opls_135 & 6 & P1E & $\mathrm{C} 8$ & 19 & -0.18 & $12.011 ;$ qtot -0.1 \\
\hline 129 & opls_140 & 6 & P1E & H81 & 19 & 0.06 & $1.008 ;$ qtot -0.12 \\
\hline 130 & opls_140 & 6 & P1E & H82 & 19 & 0.06 & $1.008 ;$ qtot -0.06 \\
\hline 131 & opls_140 & 6 & P1E & H83 & 19 & 0.06 & $1.008 ;$ qtot 0 \\
\hline
\end{tabular}

[ bonds ] 


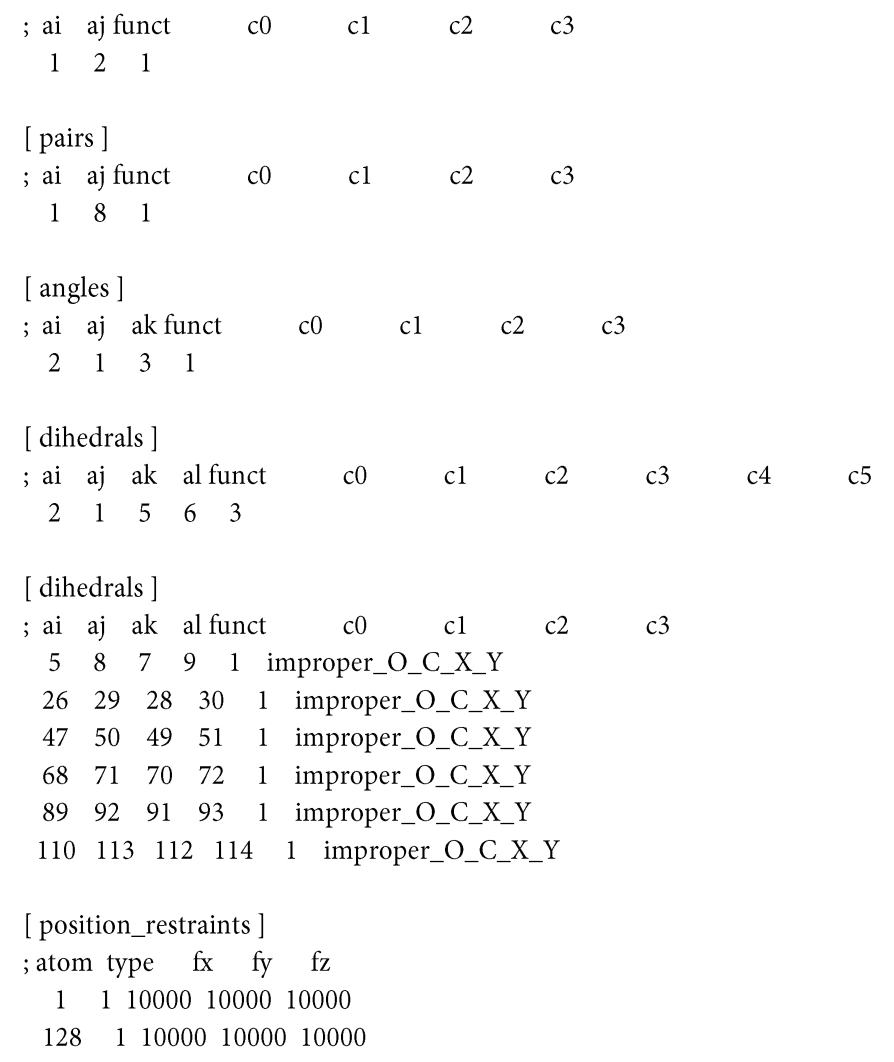

\section{Toluene}

\begin{tabular}{|c|c|c|c|c|c|c|}
\hline \multicolumn{7}{|l|}{ [ moleculetype ] } \\
\hline \multicolumn{7}{|c|}{; Name nrexcl } \\
\hline \multicolumn{7}{|l|}{ tol 3} \\
\hline \multicolumn{7}{|c|}{ [ atoms ] } \\
\hline \multicolumn{3}{|c|}{$\begin{array}{l}\text {; } \mathrm{nr} \text { type resnr residue atom } \\
\text {; residue } 1 \text { TOL } \mathrm{rtp} \text { TOL q } 0.0\end{array}$} & cgnr & r charg & mas & ass typeB ch \\
\hline 1 opls_148 & 1 TOL & $\mathrm{Cl}$ & 1 & -0.065 & 12.011 & ; qtot -0.065 \\
\hline 2 opls_140 & 1 TOL & H11 & 1 & 0.06 & 1.008 & ; qtot -0.005 \\
\hline 3 opls_140 & $1 \mathrm{TOL}$ & H12 & 1 & 0.06 & $1.008 ;$ & ; qtot 0.055 \\
\hline 4 opls_140 & $1 \mathrm{TOL}$ & $\mathrm{H} 13$ & 1 & 0.06 & $1.008 ;$ & ; qtot 0.115 \\
\hline 5 opls_145 & $1 \mathrm{TOL}$ & $\mathrm{C} 2$ & 2 & -0.115 & 12.011 & $;$ qtot 0 \\
\hline 6 opls_145 & $1 \mathrm{TOL}$ & $\mathrm{C} 3$ & 3 & -0.115 & 12.011 & ; qtot -0.115 \\
\hline 7 opls_146 & 1 TOL & $\mathrm{H} 31$ & 3 & 0.115 & 1.008 & ; qtot 0 \\
\hline 8 opls_145 & $1 \mathrm{TOL}$ & $\mathrm{C} 4$ & 4 & -0.115 & 12.011 & ; qtot -0.115 \\
\hline 9 opls_146 & $1 \mathrm{TOL}$ & H41 & 4 & 0.115 & 1.008 & ; qtot 0 \\
\hline 10 opls_145 & 1 TOL & $\mathrm{C} 5$ & 5 & -0.115 & 12.011 & ; qtot -0.115 \\
\hline 11 opls_146 & $1 \mathrm{TOL}$ & H51 & 5 & 0.115 & 1.008 & $3 ;$ gtot 0 \\
\hline 12 opls_145 & 1 TOL & C6 & 6 & -0.115 & 12.011 & ; q tot -0.115 \\
\hline 13 opls_146 & 1 TOL & $\mathrm{H} 61$ & 6 & 0.115 & 1.008 & $3 ;$ qtot 0 \\
\hline 14 opls_145 & 1 TOL & C7 & 7 & -0.115 & 12.011 & ; qtot -0.115 \\
\hline 15 opls_146 & 1 TOL & H71 & 7 & 0.115 & 1.008 & ; qtot 0 \\
\hline
\end{tabular}

[ bonds ]

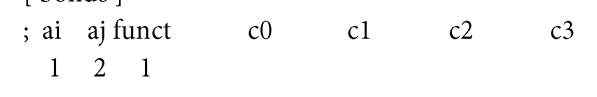

[ pairs ]

$\begin{array}{llllll}\text {; ai aj funct } & \text { c0 } & \text { cl } & \text { c2 } & \text { c3 }\end{array}$

[ angles ]

$\begin{array}{lllllll}\text {; ai aj ak funct } & \text { c0 } & \text { cl } & \text { c2 } & \text { c3 }\end{array}$

$\begin{array}{llll}2 & 1 & 3 & 1\end{array}$ 
[ dihedrals ]

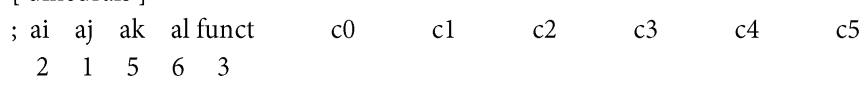

[ dihedrals ]

; ai aj ak al funct $\quad c 0 \quad c 1 \quad$ c2 $\quad c 3$

$\begin{array}{lllllll}1 & 5 & 6 & 7 & 1 & \text { improper_Z_CA_X_Y }\end{array}$

$\begin{array}{lllllll}1 & 5 & 14 & 12 & 1 & \text { improper_Z_CA_X_Y }\end{array}$

$\begin{array}{lllllll}5 & 6 & 8 & 9 & 1 & \text { improper_Z_CA_X_Y }\end{array}$

$\begin{array}{lllllll}6 & 8 & 10 & 11 & 1 & \text { improper_Z_CA_X_Y }\end{array}$

$\begin{array}{llllll}8 & 10 & 12 & 13 & 1 & \text { improper_Z_CA_X_Y }\end{array}$

$\begin{array}{llllll}10 & 12 & 14 & 15 & 1 & \text { improper_Z_CA_X_Y }\end{array}$

\section{Benzoic Acid}

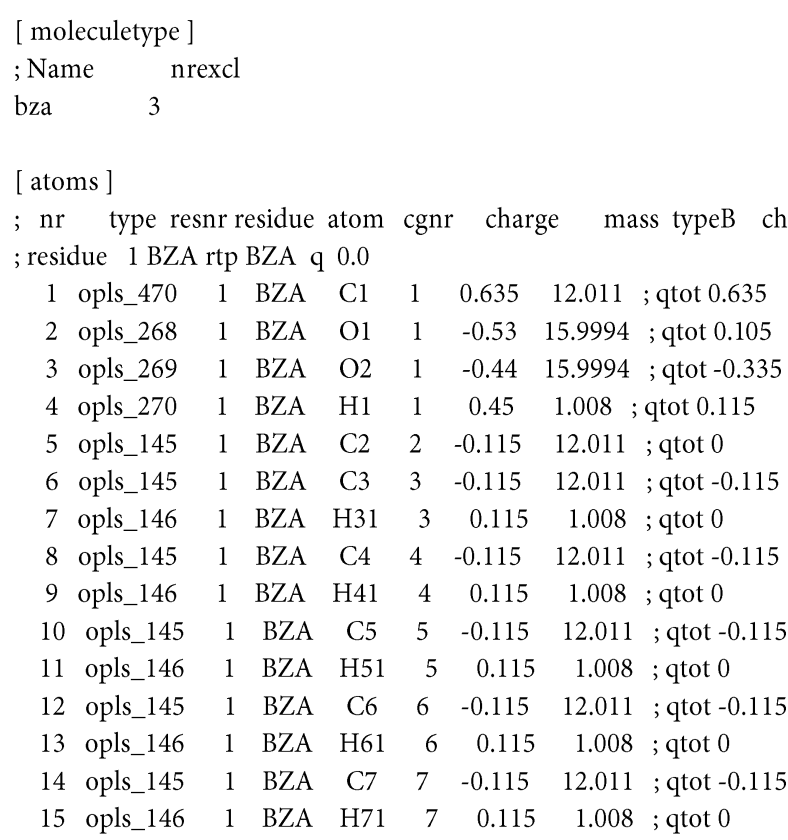

[ bonds ]

$\begin{array}{rllllll}\text {; ai } & \text { aj funct } & \text { c0 } & \text { cl } & c 2 & \text { c3 } \\ 1 & 2 & 1 & & & & \end{array}$

[ pairs ]

$\begin{array}{rrrrrrr}\text {; ai } & \text { aj funct } & \text { c0 } & \text { c1 } & \text { c2 } & \text { c3 } \\ 1 & 7 & 1 & & & & \end{array}$

[ angles ]

; ai aj ak funct $\quad$ c0 $\quad$ c1 $\quad$ c2 $\quad$ c3

$\begin{array}{llll}2 & 1 & 3 & 1\end{array}$

[ dihedrals ]

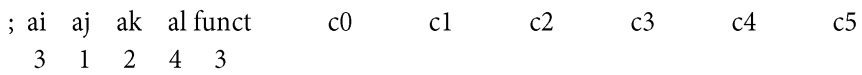

[ dihedrals ]

; ai aj ak al funct $\quad$ c0 cl $\quad$ c2 $\quad$ c3

$\begin{array}{lllllll}1 & 5 & 6 & 7 & 1 & \text { improper_Z_CA_X_Y }\end{array}$

$\begin{array}{llllll}1 & 5 & 14 & 12 & 1 & \text { improper_Z_CA_X_Y }\end{array}$

$\begin{array}{lllllll}2 & 5 & 1 & 3 & 1 & \text { improper_O_C_X_Y }\end{array}$

$\begin{array}{llllll}5 & 6 & 8 & 9 & 1 & \text { improper_Z_CA_X_Y }\end{array}$

$\begin{array}{lllllll}6 & 8 & 10 & 11 & 1 & \text { improper_Z_CA_X_Y }\end{array}$

$\begin{array}{lllllll}8 & 10 & 12 & 13 & 1 & \text { improper_Z_CA_X_Y }\end{array}$

$\begin{array}{llllll}10 & 12 & 14 & 15 & 1 & \text { improper_Z_CA_X_Y }\end{array}$ 


\section{Structural Elucidation of Synthetic Indanes Data}

\section{Exemplary ORCA input files geometry optimization and numerical frequencies}

Optimization:

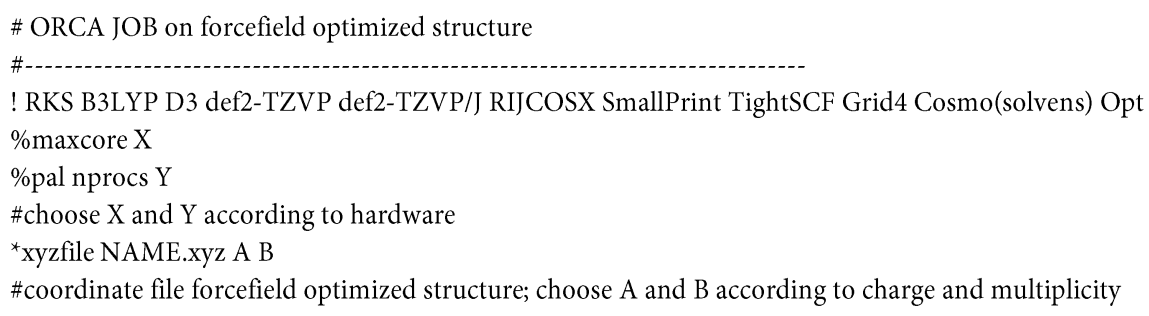

Numerical calculation of vibrational frequencies:

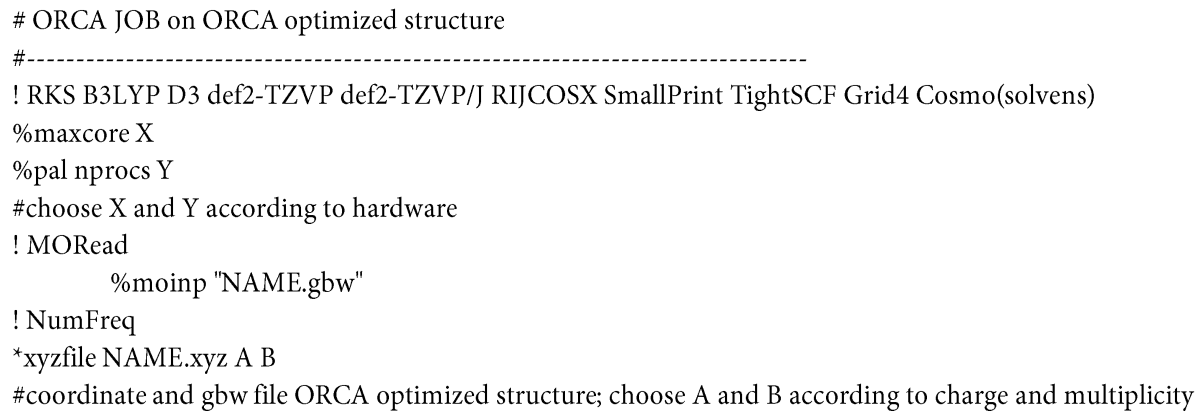




\section{Structure Data $\beta$-aa-H*}

\begin{tabular}{|c|c|c|c|}
\hline B3LYP/def2-TZVP & MM2 & MMFF9 & unoptimized \\
\hline C - $2.92510 .3532-0.2972$ & C - $2.82230 .3442-0.3608$ & C - $2.91090 .3597-0.3481$ & C - $2.86020 .4367-0.1465$ \\
\hline C - $2.9267-1.0442-0.3460$ & C $-2.8382-1.0030-0.3722$ & C - $2.8864-1.0480-0.3925$ & C - $2.8603-0.9673-0.1465$ \\
\hline C - $1.7014-1.7187-0.3393$ & C - $1.6704-1.6698-0.3095$ & C - $1.6593-1.7217-0.3461$ & C - $1.6589-1.6794-0.1402$ \\
\hline C - $0.5158-1.0018-0.2859$ & $C-0.5216-0.9845-0.2380$ & C - $0.4908-0.9773-0.2914$ & C - $0.4730-0.9652-0.1465$ \\
\hline C $-0.52890 .3891-0.2372$ & C $-0.51940 .3531-0.2348$ & C - $0.51650 .4224-0.2572$ & C $-0.47290 .4341-0.1465$ \\
\hline C - $1.73361 .0751-0.2413$ & C - $1.67151 .0334-0.2912$ & C - $1.72751 .1004-0.2773$ & C - $1.65861 .1486-0.1401$ \\
\hline C $-4.2181-1.8149-0.4316$ & C - $-4.1548-1.7396-0.4516$ & C - $-4.1765-1.8147-0.4318$ & C - $4.1610-1.7083-0.1533$ \\
\hline C $0.8981-1.5181-0.2607$ & C $0.8707-1.5475-0.1631$ & C $0.9121-1.4931-0.2009$ & C $0.9250-1.4975-0.1036$ \\
\hline C $1.7266-0.2773-0.6327$ & C $1.7107-0.3163-0.5798$ & C $1.7134-0.2457-0.6187$ & C $1.7268-0.2657-0.5483$ \\
\hline C $0.88120 .9648-0.1534$ & C $0.87460 .9168-0.1477$ & C $0.88780 .9855-0.1501$ & C $0.92520 .9662-0.1035$ \\
\hline N $1.15382 .1025-1.0286$ & N $1.04482 .0296-1.0937$ & N $1.08622 .1291-1.0467$ & N $1.09872 .0645-1.0153$ \\
\hline C $3.1249-0.2974-0.0835$ & C $3.1442-0.3238-0.0849$ & C $3.1122-0.2984-0.0700$ & C $3.0858-0.26590 .1075$ \\
\hline H $0.70432 .9389-0.6712$ & H $0.48852 .8453-0.7855$ & H $0.48302 .8964-0.7579$ & H $0.43392 .8038-0.7874$ \\
\hline H $0.77011 .9348-1.9532$ & Н $0.65121 .7715-2.0151$ & Н $0.78701 .8731-1.9874$ & Н $0.83941 .7692-1.9566$ \\
\hline H $4.86560 .3451-0.4389$ & H $4.79870 .5410-0.3754$ & H $4.88070 .1042-0.4476$ & H 4.74710 .52450 .3755 \\
\hline O $3.99470 .3621-0.8706$ & O $3.89400 .5959-0.7247$ & O $4.03350 .1677-0.9352$ & O $3.95010 .7241-0.1438$ \\
\hline O $3.4611-0.82470 .9527$ & O $3.6198-1.04510 .7578$ & O $3.4559-0.71821 .0232$ & O $3.3935-1.15980 .8595$ \\
\hline C 1.18601 .38311 .2896 & C 1.17061 .38681 .2833 & C 1.15891 .42781 .2943 & C 1.33971 .52641 .2507 \\
\hline H -3.8700 $0.8840-0.3059$ & H -3.7752 $0.8976-0.4084$ & H -3.8640 $0.8853-0.3604$ & H -3.8158 $0.9814-0.1516$ \\
\hline H - $1.6838-2.8025-0.3832$ & H - $1.6426-2.7705-0.3144$ & H - $1.6185-2.8073-0.3510$ & H - $1.6551-2.7793-0.1305$ \\
\hline H - $1.75782 .1585-0.2075$ & H - $1.67282 .1346-0.2793$ & H - $1.76792 .1837-0.2289$ & $\mathrm{H}-1.65462 .2485-0.1302$ \\
\hline H -4.1936 -2.6962 0.2119 & $\mathrm{H}-4.0283-2.8453-0.4495$ & H $-4.0126-2.8535-0.7369$ & H -3.9645-2.8038 -0.1523 \\
\hline H -5.0670 - $1.1982-0.1357$ & H -4.7987 -1.4751 0.4180 & H -4.6432 -1.8131 0.5580 & H -4.7471 -1.4362 0.7529 \\
\hline H $-4.3992-2.1678-1.4514$ & H - $-4.6944-1.4664-1.3869$ & H - $4.8660-1.3665-1.1548$ & H - $4.7381-1.4360-1.0652$ \\
\hline H $1.0679-2.3446-0.9515$ & H $1.0207-2.4162-0.8428$ & H $1.0869-2.3227-0.8932$ & H $1.0591-2.3475-0.8095$ \\
\hline Н $1.1709-1.86860 .7378$ & H $1.0660-1.85320 .8903$ & H $1.1274-1.82120 .8214$ & H $1.2278-1.90700 .8860$ \\
\hline H $1.7934-0.1927-1.7180$ & H $1.7551-0.3322-1.6992$ & H $1.7770-0.2654-1.7184$ & H $1.8829-0.2657-1.6503$ \\
\hline H 2.19321 .79491 .3735 & Н 2.19971 .80281 .3675 & Н 2.17151 .83131 .4091 & H 1.64522 .59031 .1349 \\
\hline H 0.47422 .14991 .6024 & H 0.46442 .19071 .5926 & H 0.45932 .21101 .6117 & H 0.48151 .46211 .9566 \\
\hline H 1.09530 .53931 .9723 & H 1.07690 .55282 .0151 & H 1.04750 .59662 .0008 & H 2.19470 .93631 .6501 \\
\hline
\end{tabular}

RS-ax

\begin{tabular}{|c|c|c|c|}
\hline B3LYP/def2-TZVP & MM2 & MMFF9 & unoptimized \\
\hline C - 1.98690 .98570 .7836 & C - 1.87010 .96030 .7311 & C - 1.94200 .99350 .7360 & C - 1.73091 .09620 .7185 \\
\hline C - $1.8547-0.40620 .7849$ & C - $1.7482-0.38120 .7533$ & C - $1.8236-0.40890 .7896$ & C - $1.7309-0.30780 .7185$ \\
\hline C - $0.6028-1.01600 .7264$ & C - $0.5438-0.97640 .7483$ & C - $0.5704-1.02870 .7686$ & C - $0.5295-1.01980 .7248$ \\
\hline C $0.5344-0.22270 .6624$ & C $0.5552-0.21150 .7156$ & C $0.5647-0.23470 .6926$ & C $0.6564-0.30560 .7184$ \\
\hline C $0.4150 \quad 1.16410 .6539$ & C 0.44571 .12200 .7054 & C 0.45021 .16160 .6512 & C 0.65641 .09380 .7185 \\
\hline C -0.8299 1.76910 .7192 & C -0.75551 .71440 .7071 & C -0.78721 .78570 .6826 & C -0.52941 .80810 .7249 \\
\hline C $1.9949-0.65490 .6751$ & C $1.9954-0.65340 .7178$ & C $2.0189-0.65270 .7052$ & C $2.0544-0.83770 .7613$ \\
\hline C 2.72600 .66780 .2141 & C 2.69090 .63370 .1939 & C 2.70530 .64160 .1821 & C 2.85620 .39410 .3166 \\
\hline C 1.76821 .82040 .5898 & C 1.78781 .79730 .6705 & C 1.79931 .81620 .6089 & C 2.05441 .62600 .7614 \\
\hline C $2.98380 .6543-1.2704$ & C $2.80420 .6459-1.3261$ & C $2.88330 .6554-1.3223$ & C $3.01920 .3942-1.1835$ \\
\hline O $4.23180 .2390-1.5620$ & O $4.05640 .3640-1.7387$ & O $4.08170 .1633-1.7015$ & O $3.69001 .3842-1.7835$ \\
\hline O $2.18810 .9631-2.1268$ & O $1.92130 .8735-2.1179$ & O $2.09951 .0523-2.1673$ & O $2.5441-0.4997-1.8428$ \\
\hline N $2.4752-0.96312 .0404$ & N $2.3941-0.89042 .1159$ & N $2.4239-0.90992 .1021$ & $\mathrm{~N} 2.4194-1.25222 .0890$ \\
\hline C $2.2713-1.8775-0.1911$ & C $2.2713-1.9125-0.1128$ & C $2.3108-1.9150-0.1106$ & C $2.2794-2.0742-0.0990$ \\
\hline C -3.3448 1.63460 .8227 & C -3.2260 1.6262 0.7339 & C -3.2934 1.64400 .7895 & C -3.0315 1.8374 0.7115 \\
\hline H - $2.7454-1.02210 .8306$ & H -2.6510 - 1.01380 .7784 & H - $2.7165-1.02770 .8501$ & H - $2.6866-0.85240 .7134$ \\
\hline H -0.5305 -2.0974 0.7322 & H - $0.4636-2.07420 .7741$ & H - $0.4996-2.11040 .8179$ & H - $0.5256-2.11970 .7345$ \\
\hline H - 0.91362 .85050 .7108 & H - 0.82142 .81360 .6885 & Н - 0.85732 .86930 .6580 & H - 0.52542 .90810 .7348 \\
\hline
\end{tabular}




\begin{tabular}{|c|c|c|c|}
\hline H 3.68500 .73110 .7223 & H 3.71830 .74300 .6154 & H 3.70480 .78670 .6143 & H 3.87370 .39400 .7676 \\
\hline H $1.80512 .6324-0.1360$ & H $1.81492 .6787-0.0077$ & H $1.82262 .6557-0.0932$ & H 2.18872 .47610 .0557 \\
\hline Н 2.04672 .24191 .5594 & H 2.05522 .11861 .7042 & H 2.06332 .17081 .6112 & H 2.35732 .03531 .7511 \\
\hline H $4.32610 .2184-2.5295$ & H $4.06450 .3799-2.7098$ & H $4.06780 .2320-2.6796$ & H $3.67131 .1848-2.7347$ \\
\hline H $1.9596-1.76132 .3998$ & H $1.9094-1.72682 .4838$ & H $1.8854-1.70032 .4577$ & H $1.7631-1.96112 .4164$ \\
\hline Н $2.2599-0.19202 .6658$ & H $2.0646-0.10982 .7098$ & H $2.1456-0.11782 .6827$ & H $2.2959-0.47232 .7347$ \\
\hline H $1.7365-2.74060 .2102$ & H $1.8083-2.81360 .3488$ & H $1.8332-2.79980 .3284$ & H $2.6493-2.90810 .5385$ \\
\hline Н $1.9300-1.7259-1.2153$ & H $1.8703-1.8284-1.1476$ & H $1.9310-1.8365-1.1355$ & H $1.3199-2.3717-0.5782$ \\
\hline Н $3.3357-2.1130-0.1948$ & H $3.3665-2.1041-0.1778$ & Н $3.3871-2.1158-0.1586$ & H $3.0330-1.8464-0.8857$ \\
\hline H -4.1038 $0.9422 \quad 1.1883$ & H -4.0645 0.89450 .7331 & H -4.0823 0.94420 .4944 & H -3.8737 1.10970 .7070 \\
\hline H -3.3440 2.5138 1.4691 & H -3.3395 2.2652 1.6391 & H -3.5000 1.99451 .8054 & H -3.0987 2.4762 1.6204 \\
\hline H -3.6492 $1.9677-0.1741$ & H -3.3429 2.2686-0.1685 & H -3.3384 2.4945 0.1011 & H -3.0891 $2.4768-0.1976$ \\
\hline
\end{tabular}

SS-eq

\begin{tabular}{|c|c|c|c|}
\hline B3LYP/def2-TZVP & MM2 & MMFF9 & unoptimized \\
\hline C - $2.78150 .3272-0.0226$ & C - $2.77370 .2922-0.1036$ & C - $2.84500 .3121-0.0849$ & C - $2.86020 .3204-0.2065$ \\
\hline C - $-2.8209-1.0664-0.1415$ & C - $2.8091-1.0542-0.0920$ & C -2.8563 - $1.0959-0.1188$ & C - $-2.8603-1.0836-0.2065$ \\
\hline C - $1.6151-1.7705-0.1897$ & C - $1.6480-1.7333-0.0492$ & C - $1.6452-1.7991-0.0879$ & C - $1.6589-1.7957-0.2002$ \\
\hline C - $0.4084-1.0864-0.1355$ & C - $0.4891-1.0615-0.0228$ & C - $0.4578-1.0833-0.0627$ & C - $0.4730-1.0815-0.2065$ \\
\hline C $-0.38480 .3000-0.0393$ & C - $0.46300 .2756-0.0456$ & C - $0.44790 .3166-0.0448$ & C $-0.47290 .3178-0.2065$ \\
\hline C - 1.57241 .01440 .0350 & C - $1.61150 .9652-0.0786$ & C - $1.64211 .0229-0.0442$ & C - $1.65861 .0322-0.2001$ \\
\hline C $-4.1350-1.7991-0.2074$ & C -4.1366 -1.7745 - 0.1197 & C $-4.1645-1.8318-0.1214$ & C $-4.1610-1.8247-0.2133$ \\
\hline C $0.9853-1.6511-0.1587$ & C $0.8904-1.65010 .0303$ & C $0.9323-1.62960 .0050$ & C $0.9250-1.6138-0.1636$ \\
\hline C $1.8630-0.4307-0.4773$ & C $1.7616-0.4433-0.3930$ & C $1.7585-0.4089-0.4453$ & C $1.7268-0.3821-0.6083$ \\
\hline C $1.02850 .8549-0.0174$ & C $0.94450 .8225-0.0082$ & C 0.96990 .85140 .0311 & C $0.92520 .8499-0.1635$ \\
\hline N 1.32571 .34581 .3310 & N 1.25941 .24431 .3675 & N 1.23511 .28231 .4083 & N 1.29021 .26411 .1643 \\
\hline C $3.2143-0.47320 .1704$ & C $3.1847-0.53300 .1294$ & C $3.1809-0.54530 .0294$ & C $3.0858-0.38220 .0475$ \\
\hline O $4.11840 .2831-0.4868$ & O 3.84930 .63090 .0034 & O 3.86080 .61390 .0891 & O $3.95010 .6078-0.2038$ \\
\hline O $3.4888-1.06661 .1883$ & O $3.7217-1.50840 .5965$ & O $3.7588-1.59290 .2748$ & O $3.3935-1.27620 .7995$ \\
\hline C $1.22362 .0061-0.9998$ & C $1.14811 .9904-0.9848$ & C $1.18992 .0413-0.9120$ & C $1.15032 .0864-1.0236$ \\
\hline H -3.71200.88140.0180 & H -3.71990.8582 -0.1320 & H -3.7835 $0.8630-0.0785$ & H -3.8158 0.8651-0.2116 \\
\hline H - $1.6263-2.8511-0.2825$ & $\mathrm{H}-1.6340-2.8342-0.0324$ & $\mathrm{H}-1.6317-2.8854-0.0823$ & H - $1.6551-2.8957-0.1904$ \\
\hline H -1.5639 2.0933 0.1299 & $\mathrm{H}-1.60682 .0661-0.0820$ & H - 1.64902 .10740 .0059 & H - $1.65462 .1322-0.1902$ \\
\hline H -4.9396 - $1.1393-0.5336$ & $\mathrm{H}-4.0246-2.8811-0.0833$ & H -4.0396 -2.8602 -0.4762 & H -3.9645 -2.9202 -0.2123 \\
\hline H -4.0816 -2.6422 -0.8979 & H -4.7591 -1.4729 0.7533 & H - $4.5802-1.85960 .8905$ & H - $4.7471-1.55260 .6929$ \\
\hline H -4.4102 -2.2017 0.7722 & H -4.6914 -1.5242 -1.0526 & H - $4.8801-1.3412-0.7894$ & H - $4.7381-1.5523-1.1252$ \\
\hline H $1.1140-2.4445-0.8960$ & H $1.0107-2.5240-0.6489$ & H $1.0721-2.4738-0.6775$ & H $1.0591-2.4639-0.8695$ \\
\hline H $1.2507-2.07170 .8143$ & H $1.0928-1.95581 .0831$ & H $1.1668-1.94321 .0284$ & H $1.2278-2.02330 .8260$ \\
\hline H $2.0050-0.3492-1.5546$ & Н $1.8439-0.4749-1.5089$ & H $1.8031-0.4139-1.5454$ & H $1.8829-0.3820-1.7103$ \\
\hline H 2.25681 .74741 .3787 & H 2.25151 .52451 .4529 & Н 2.21891 .53641 .4961 & H 2.28971 .46521 .1950 \\
\hline H 1.28340 .59672 .0135 & H 1.13220 .45142 .0202 & H 1.06410 .51292 .0534 & H 1.16660 .48411 .8098 \\
\hline H 4.95510 .25370 .0071 & H 4.75910 .49740 .3155 & H 4.77220 .34350 .3341 & H 4.74710 .40820 .3155 \\
\hline H $0.61042 .8574-0.7034$ & H $0.55122 .8811-0.6861$ & Н $0.57972 .9059-0.6241$ & H $1.52032 .9202-0.3860$ \\
\hline H $2.26812 .3258-1.0169$ & H $2.21082 .3166-1.0170$ & H $2.23472 .3689-0.9158$ & H $1.90451 .8585-1.8098$ \\
\hline Н $0.93751 .7018-2.0064$ & Н $0.84711 .7121-2.0202$ & Н $0.91741 .7884-1.9437$ & Н $0.19122 .3840-1.5035$ \\
\hline
\end{tabular}

SS-ax

\begin{tabular}{llll}
\hline B3LYP/def2-TZVP & MM2 & MMFF9 & unoptimized \\
\hline C -1.82400 .83520 .7568 & C -1.79000 .69780 .6095 & C -1.84940 .71960 .5821 & C -1.73080 .87340 .6749 \\
C $-1.7578-0.56120 .7448$ & C $-1.7616-0.64870 .6485$ & C $-1.8330-0.68820 .6367$ & C $-1.7309-0.53070 .6749$ \\
C $-0.5355-1.22820 .6831$ & C $-0.6011-1.32540 .6585$ & C $-0.6275-1.39530 .6353$ & C $-0.5296-1.24270 .6812$ \\
C $0.6341-0.48540 .6310$ & C $0.5462-0.63550 .6260$ & C $0.5612-0.68410 .5788$ & C $0.6563-0.52860 .6748$ \\
C 0.58410 .90390 .6455 & C 0.53160 .70160 .5952 & C 0.54950 .71570 .5322 & C 0.65640 .87080 .6749 \\
C - 0.63181 .56540 .7084 & C -0.62651 .37440 .5825 & C -0.63981 .42750 .5472 & C -0.52931 .58520 .6813 \\
C $2.0594-1.00150 .6005$ & C $1.9490-1.18470 .6353$ & C $1.9782-1.20760 .6102$ & C $2.0543-1.06080 .7177$ \\
\hline
\end{tabular}




\begin{tabular}{|c|c|c|c|}
\hline C 2.87290 .31310 .1961 & C 2.73980 .04580 .1097 & C 2.75770 .03170 .0620 & C 2.85610 .17090 .2730 \\
\hline C 1.96671 .49710 .5945 & C 1.91921 .27800 .5631 & C 1.94461 .26500 .5181 & C 2.05451 .40280 .7179 \\
\hline C $3.13900 .3024-1.2823$ & C $2.87480 .0798-1.4085$ & C $2.91060 .0767-1.4475$ & C $3.01920 .1710-1.2271$ \\
\hline O $4.3799-0.1397-1.5704$ & O $3.94790 .8020-1.7893$ & O $3.58821 .1708-1.8602$ & O $3.69001 .1610-1.8271$ \\
\hline O $2.34890 .6138-2.1436$ & O $2.1568-0.4475-2.2233$ & O $2.5730-0.7625-2.2657$ & O $2.5440-0.7228-1.8863$ \\
\hline N $2.1812-2.1476-0.2924$ & N $2.0729-2.3600-0.2422$ & N $2.1380-2.4617-0.1254$ & N $2.2276-2.1591-0.1942$ \\
\hline C $2.5005-1.44002 .0023$ & C $2.3695-1.56122 .0645$ & C $2.3836-1.49382 .0690$ & C $2.4687-1.62122 .0719$ \\
\hline C - 3.14871 .54750 .8287 & C - 3.09741 .45490 .5960 & C - -3.15281 .46230 .6248 & C -3.0314 1.61460.6679 \\
\hline H - $2.6770-1.13460 .7773$ & H - $2.7057-1.21760 .6737$ & H - $-2.7675-1.24320 .6845$ & H -2.6867 -1.0752 0.6699 \\
\hline H -0.4986 -2.31050.6648 & $\mathrm{H}-0.5890-2.42620 .6935$ & H - $-0.6212-2.48040 .6819$ & H - $0.5258-2.34270 .6909$ \\
\hline H -0.6650 2.64960.7064 & $\mathrm{H}-0.61732 .47520 .5504$ & H - 0.63252 .51340 .5302 & H - 0.52522 .68520 .6912 \\
\hline H 3.83080 .32740 .7112 & Н 3.77190 .05690 .5331 & Н 3.77940 .05460 .4631 & Н 3.87370 .17080 .7240 \\
\hline H $2.04122 .3270-0.1084$ & H $2.00682 .1431-0.1311$ & Н $2.02492 .1231-0.1558$ & Н 2.18882 .25300 .0121 \\
\hline H $2.26311 .8780 \quad 1.5754$ & Н 2.20451 .59891 .5917 & Н 2.22961 .57101 .5304 & Н 2.35741 .81221 .7075 \\
\hline Н $4.4744-0.1664-2.5379$ & Н $3.98390 .8055-2.7598$ & Н $3.63681 .0647-2.8343$ & Н $3.67140 .9616-2.7782$ \\
\hline H $3.1445-2.4525-0.3751$ & H $3.0425-2.4752-0.5834$ & Н $3.1197-2.7309-0.1474$ & Н $3.2186-2.3907-0.2631$ \\
\hline H $1.8339-1.9356-1.2210$ & H $1.5000-2.2173-1.0921$ & H $1.8932-2.2908-1.1085$ & Н $1.9684-1.8636-1.1354$ \\
\hline H $3.5279-1.81301 .9825$ & Н $3.4217-1.92582 .0840$ & Н $3.4339-1.80202 .1337$ & Н $2.7741-2.68521 .9560$ \\
\hline H $2.4502-0.60632 .7039$ & H $2.2960-0.69482 .7598$ & H $2.2602-0.61472 .7122$ & Н $3.3242-1.03152 .4707$ \\
\hline H $1.8484-2.23632 .3613$ & H $1.7243-2.37422 .4691$ & Н $1.7728-2.29402 .5059$ & Н $1.6110-1.55642 .7782$ \\
\hline H -3.97130.8784 0.5746 & H -3.98390.78220.5907 & H -3.9734 0.84070.2514 & H -3.8737 0.8871 0.6634 \\
\hline H -3.3315 1.93761 .8346 & H -3.1749 2.1051 1.4970 & Н -3.3789 1.76211 .6527 & H -3.0986 2.2535 1.5768 \\
\hline H -3.1762 2.39850.1458 & H -3.1621 $2.0988-0.3106$ & H -3.1093 2.3562 -0.0063 & H -3.0889 2.2541 -0.2412 \\
\hline
\end{tabular}

\section{Structure Data $\beta$-aa-Me*}

\begin{tabular}{|c|c|c|c|}
\hline B3LYP/def2-TZVP & MM2 & MMFF9 & unoptimized \\
\hline C - $2.73990 .2892-0.4703$ & C - $2.67300 .2478-0.4685$ & C - $2.75210 .2246-0.5278$ & C - 2.33241 .05910 .2337 \\
\hline C - $2.7775-0.67890 .5424$ & C - $-2.7095-0.65760 .5273$ & C - $-2.7632-0.73760 .4997$ & C - $1.94131 .5725-1.0132$ \\
\hline C - $1.5852-1.03001 .1777$ & C - $1.5636-0.97551 .1575$ & C - $1.5759-1.03991 .1805$ & C $-0.73321 .1838-1.5960$ \\
\hline C - $0.3898-0.42690 .8020$ & C $-0.4132-0.39760 .7836$ & C -0.3995 -0.4104 0.7962 & C $0.07040 .2915-0.9071$ \\
\hline C - $-0.36990 .5264-0.2079$ & C - $-0.38890 .4972-0.2110$ & C - $-0.39310 .5377-0.2401$ & C $-0.3194-0.22020 .3356$ \\
\hline C - $1.54710 .8957-0.8461$ & C - $1.52290 .8319-0.8416$ & C - $1.56900 .8679-0.8991$ & C - 1.52090 .14960 .9154 \\
\hline C $0.9902-0.65511 .3738$ & C $0.9535-0.61961 .3746$ & C $0.9762-0.55371 .3943$ & C $1.3773-0.2962-1.3384$ \\
\hline C $1.8998-0.01930 .3035$ & C 1.87080 .00280 .2902 & C 1.83520 .00270 .2342 & C $1.9147-0.8383-0.0060$ \\
\hline C $1.02511 .0684-0.4600$ & C $0.99611 .0396-0.4684$ & C $0.99211 .1256-0.4366$ & C $0.6910-1.19720 .8495$ \\
\hline C $1.36391 .1421-1.9461$ & C $1.32481 .1208-1.9670$ & C $1.36961 .2980-1.9116$ & C $0.9663-0.98652 .3325$ \\
\hline C 3.18390 .53660 .8486 & C 3.18640 .52460 .8433 & C 3.18460 .46770 .7055 & C $2.7584-2.0660-0.2470$ \\
\hline C $1.3196-2.11351 .6827$ & C $1.2844-2.08581 .6753$ & C $1.3588-1.98231 .7646$ & C $2.30060 .7654-1.9215$ \\
\hline O $4.02540 .9211-0.1345$ & O $3.90151 .1792-0.0911$ & O $4.13850 .3326-0.2365$ & O $3.3267-2.70030 .7850$ \\
\hline O 3.46950 .65902 .0170 & O 3.60860 .39151 .9665 & O 3.46190 .93721 .7964 & O $2.9206-2.4762-1.3716$ \\
\hline N 1.10962 .42780 .0876 & N 1.11612 .36980 .1532 & N 1.03582 .43890 .2147 & N $0.2849-2.57320 .7511$ \\
\hline H -3.6600 0.5684 -0.9709 & H -3.6072 $0.5219-0.9869$ & H -3.6771 $0.4872-1.0374$ & C - $-2.82812 .5461-1.7250$ \\
\hline C - $4.0830-1.32480 .9304$ & C $-4.0232-1.28940 .9238$ & C - $4.0564-1.37960 .9108$ & H -3.2874 1.37630 .6780 \\
\hline H - $1.5989-1.77801 .9618$ & H - $1.5658-1.70321 .9830$ & H - $1.5795-1.75301 .9999$ & H - $0.42801 .5765-2.5772$ \\
\hline H - $1.54121 .6426-1.6301$ & $\mathrm{H}-1.51731 .5780-1.6512$ & H - $1.57951 .6263-1.6759$ & H - $1.8285-0.26231 .8879$ \\
\hline H $1.0890-0.08212 .3009$ & Н $0.9954-0.01392 .3124$ & H 1.04170 .08412 .2844 & H $1.2911-1.0618-2.1418$ \\
\hline H $2.1687-0.7828-0.4308$ & Н $2.1361-0.8117-0.4285$ & H $2.0033-0.8142-0.4857$ & Н $2.5535-0.08370 .5051$ \\
\hline H $0.67701 .8245-2.4461$ & Н $0.68801 .8722-2.4853$ & Н $0.72582 .0304-2.4133$ & Н $0.7764-1.93442 .8840$ \\
\hline H $2.37921 .5143-2.0958$ & Н $2.37991 .4273-2.1388$ & H $2.40411 .6425-2.0207$ & H $2.0278-0.68252 .4727$ \\
\hline H $1.27730 .1594-2.4096$ & H $1.17000 .1399-2.4710$ & H $1.27100 .3556-2.4630$ & Н $0.2962-0.18892 .7243$ \\
\hline Н $0.6378-2.51532 .4337$ & Н $0.6391-2.49442 .4853$ & Н $0.7301-2.35812 .5790$ & H $1.88111 .1347-2.8840$ \\
\hline H $2.3341-2.21102 .0746$ & Н $2.3403-2.19172 .0132$ & Н $2.3999-2.03132 .1001$ & Н $3.30630 .3236-2.1010$ \\
\hline
\end{tabular}




\begin{tabular}{|c|c|c|c|}
\hline H $1.2311-2.72820 .7840$ & Н $1.1479-2.72540 .7740$ & H $1.2422-2.66570 .9162$ & Н $2.38931 .6133-1.2060$ \\
\hline H 4.82291 .29210 .2791 & H 4.74441 .45240 .3063 & H 4.95720 .65910 .1937 & H $3.8151-3.44980 .4048$ \\
\hline H 0.89282 .44401 .0783 & Н 0.90572 .30991 .1647 & H 0.75902 .34761 .1935 & H $0.0795-2.7983-0.2223$ \\
\hline H $2.03972 .8149-0.0345$ & Н 2.08542 .72540 .0862 & H 1.98582 .80660 .2246 & H $1.0703-3.17750 .9928$ \\
\hline H -3.9386 -2.0673 1.7151 & H -3.9149-2.0170 1.7589 & H -3.8809 -2.3608 1.3641 & H - $2.35772 .8405-2.6898$ \\
\hline H - $4.7956-0.58211 .2972$ & $\mathrm{H}-4.7444-0.50701 .2531$ & H -4.5755 - -0.74451 .6350 & H - $3.81512 .0716-1.9236$ \\
\hline H - $4.5442-1.82510 .0751$ & $\mathrm{H}-4.4650-1.83590 .0596$ & H $-4.7057-1.53460 .0428$ & H - $2.97083 .4498-1.0911$ \\
\hline
\end{tabular}

SSS- $a x$

\begin{tabular}{|c|c|c|c|}
\hline B3LYP/def2-TZVP & MM2 & MMFF9 & unoptimized \\
\hline C - $2.33640 .0138-0.8948$ & C - $2.2270-0.0209-0.9261$ & C - $2.2821-0.0070-0.9699$ & C - 2.08291 .58110 .8278 \\
\hline C - $2.1862-0.92730 .1278$ & C - $2.1270-0.89000 .0982$ & C - $2.2040-0.93710 .0826$ & C - $1.69182 .0945-0.4191$ \\
\hline C - $0.9288-1.28850 .6001$ & C - $0.9349-1.21520 .6247$ & C -0.9711 -1.2961 0.6325 & C - $0.48371 .7058-1.0019$ \\
\hline C $0.1932-0.69910 .0373$ & C $0.1725-0.66210 .1127$ & C $0.1855-0.72220 .1218$ & C $0.31990 .8135-0.3130$ \\
\hline C $0.06550 .2338-0.9833$ & C $0.08630 .2041-0.9022$ & C $0.11320 .2074-0.9200$ & C - 0.06990 .30180 .9297 \\
\hline C - $1.19120 .5948-1.4521$ & C - $1.10400 .5278-1.4253$ & C - $1.10850 .5723-1.4709$ & C - 1.27140 .67161 .5095 \\
\hline C $1.6303-0.91450 .4575$ & C $1.5902-0.88880 .5660$ & C $1.6071-0.92870 .5866$ & C $1.62680 .2258-0.7443$ \\
\hline C $2.4313-0.1385-0.6931$ & C $2.3967-0.2433-0.5976$ & C $2.4169-0.2897-0.5905$ & C $2.1642-0.31630 .5881$ \\
\hline C $1.40640 .7709-1.4253$ & C $1.43070 .7214-1.3362$ & C $1.47310 .7060-1.3215$ & C $0.9405-0.67521 .4436$ \\
\hline C $1.58012 .2564-1.0923$ & C $1.62542 .1947-0.9580$ & C $1.70982 .1601-0.9296$ & C $0.4967-2.11281 .2066$ \\
\hline C $3.0607-1.1407-1.6181$ & C $2.9198-1.2659-1.6006$ & C $2.9236-1.2743-1.6266$ & C 2.99210 .73741 .2817 \\
\hline N $1.9073-2.34030 .6065$ & N $1.8667-2.32790 .7179$ & N $1.8914-2.33280 .8908$ & N $2.49861 .2281-1.2948$ \\
\hline O $4.3187-1.4565-1.2430$ & O $4.1356-0.9246-2.0723$ & O $3.8531-0.7177-2.4352$ & O 3.55790 .47212 .4648 \\
\hline O $2.5254-1.6621-2.5696$ & O $2.3644-2.2720-1.9705$ & O $2.6077-2.4411-1.7839$ & O 3.14401 .82060 .7689 \\
\hline H $4.6527-2.1385-1.8498$ & Н $4.4124-1.5966-2.7166$ & Н $4.0925-1.4402-3.0543$ & C -3.38262 .01281 .4325 \\
\hline C -3.71190.3886 - 1.3808 & C - $3.56800 .3447-1.5178$ & C - $3.61080 .3815-1.5506$ & C $1.5088-0.8218-1.8435$ \\
\hline H -3.0707 -1.3806 0.5593 & H -3.0362 -1.34770.5219 & Н -3.1092 -1.3896 0.4827 & H - $-2.34352 .8099-0.9421$ \\
\hline H -0.8244 -2.0182 1.3928 & H -0.8653-1.9238 1.4652 & H - $0.9174-2.01351 .4470$ & H -0.1785 2.0985 -1.9831 \\
\hline H -1.2894 $1.3230-2.2507$ & H - $1.15541 .2419-2.2622$ & H - $1.15251 .2946-2.2818$ & H - 1.57900 .25972 .4820 \\
\hline C $1.9027-0.24871 .8112$ & C $1.8490-0.19011 .9103$ & C $1.8383-0.15391 .8972$ & H $2.8143-1.20500 .4260$ \\
\hline Н $3.23190 .4451-0.2420$ & H $3.28110 .3011-0.1913$ & Н $3.31630 .2036-0.1967$ & H $1.1167-0.61572 .5409$ \\
\hline H 2.5700 2.6064 -1.3936 & H $2.64412 .5437-1.2433$ & Н $2.73312 .4621-1.1761$ & H $1.2700-2.80991 .6002$ \\
\hline Н $0.83772 .8539-1.6236$ & Н $0.88952 .8447-1.4843$ & H $1.02642 .8232-1.4719$ & H -0.4675 -2.2942 1.7320 \\
\hline H $1.45422 .4461-0.0244$ & H 1.49972 .36410 .1339 & H 1.55062 .33830 .1372 & Н $0.3636-2.28460 .1150$ \\
\hline H $1.6971-2.8521-0.2428$ & Н $1.4669-2.8447-0.0846$ & Н $1.7525-2.88260 .0340$ & Н $2.63101 .9751-0.6130$ \\
\hline Н $2.8789-2.50500 .8454$ & H $2.8822-2.52250 .6910$ & Н $2.8760-2.45051 .1209$ & H $3.43010 .8337-1.4257$ \\
\hline H $1.2529-0.68472 .5699$ & Н $1.2144-0.62362 .7166$ & Н $1.2306-0.55922 .7164$ & H 4.03551 .27862 .7221 \\
\hline H $2.9426-0.40322 .1110$ & Н $2.9117-0.31152 .2209$ & Н $2.8891-0.20532 .2068$ & H -3.8877 2.73390 .7516 \\
\hline H 1.70990 .82361 .7640 & H 1.62880 .89891 .8653 & H 1.57160 .90351 .8089 & H -4.03551 .12381 .5810 \\
\hline H $1.51910 .6526-2.5049$ & H $1.52940 .6196-2.4438$ & H $1.57000 .6210-2.4110$ & H -3.1907 2.50142.4139 \\
\hline H -4.4805 - $0.1653-0.8423$ & H -4.4124 -0.1979-1.0373 & H $-4.4253-0.2112-1.1220$ & H $2.1161-0.5084-2.7221$ \\
\hline H -3.9033 $1.4555-1.2360$ & H -3.7576 $1.4356-1.3965$ & H -3.8154 $1.4372-1.3465$ & H $1.8821-1.7996-1.4650$ \\
\hline H -3.8267 $0.1738-2.4467$ & H -3.5905 $0.1014-2.6046$ & H -3.6130 $0.2163-2.6329$ & Н $0.4427-0.9228-2.1467$ \\
\hline
\end{tabular}

SSR-eq

\begin{tabular}{llll}
\hline B3LYP/def2-TZVP & MM2 & MMFF9 & unoptimized \\
\hline C $-2.5961-0.2124-0.8864$ & C $-2.5464-0.2766-0.8809$ & C $-2.6140-0.2774-0.9162$ & C -2.33241 .05910 .4445 \\
C $-2.6425-1.06130 .2237$ & C $-2.5833-1.03910 .2286$ & C $-2.6341-1.09570 .2297$ & C $-1.94131 .5725-0.8024$ \\
C $-1.4541-1.34640 .9031$ & C $-1.4370-1.27240 .8939$ & C $-1.4470-1.32710 .9365$ & C $-0.73321 .1838-1.3852$ \\
C $-0.2566-0.79180 .4689$ & C $-0.2881-0.75010 .4431$ & C $-0.2669-0.76280 .4707$ & C $0.07040 .2915-0.6963$ \\
C $-0.23130 .0546-0.6341$ & C $-0.26190 .0006-0.6645$ & C $-0.24740 .0327-0.6840$ & C $-0.3194-0.22020 .5464$ \\
C $-1.40120 .3528-1.3169$ & C $-1.39610 .2485-1.3333$ & C $-1.42270 .2909-1.3755$ & C -1.52090 .14961 .1262 \\
C $1.1120-0.92981 .0997$ & C $1.0743-0.91031 .0648$ & C $1.1030-0.87911 .0861$ & C $1.3773-0.2962-1.1276$ \\
C $2.0271-0.4140-0.0508$ & C $1.9805-0.4418-0.1026$ & C $1.9823-0.4498-0.1160$ & C $1.9147-0.83830 .2048$ \\
C $1.16030 .5759-0.9297$ & C $1.12310 .5035-0.9923$ & C $1.14660 .5556-0.9714$ & C $0.6910-1.19721 .0603$ \\
\hline
\end{tabular}




\begin{tabular}{|c|c|c|c|}
\hline C $1.49130 .4810-2.4179$ & C $1.47990 .3751-2.4809$ & C $1.51840 .4509-2.4572$ & C $0.9663-0.98652 .5433$ \\
\hline C 3.40430 .07210 .3149 & C $3.3798-0.03740 .3232$ & C $3.3835-0.07310 .2913$ & C $2.7584-2.0660-0.0361$ \\
\hline C $1.1534-0.19452 .4469$ & C $1.1991-0.07882 .3478$ & C $1.2201-0.03392 .3522$ & C $1.1781-1.4128-2.1441$ \\
\hline O $4.04530 .6213-0.7423$ & O $3.71131 .2166-0.0319$ & O $3.85191 .0742-0.2275$ & O $3.3267-2.70030 .9958$ \\
\hline O $3.9468-0.03101 .3891$ & O $4.1562-0.73720 .9288$ & O $4.1076-0.74261 .0134$ & O $2.9206-2.4762-1.1607$ \\
\hline N $1.22991 .9912-0.5395$ & N $1.21511 .9174-0.5902$ & N $1.21091 .9681-0.5864$ & $\mathrm{~N} 0.2849-2.57320 .9620$ \\
\hline H -3.5150 0.0131-1.4145 & H - $3.4810-0.0738-1.4303$ & H -3.5377 - $0.0728-1.4539$ & C - $2.82812 .5461-1.5141$ \\
\hline C -3.9519-1.6574 0.6652 & C -3.8969-1.6101 0.7084 & C -3.9349 - 1.66620 .7145 & H -3.2874 1.37630 .8888 \\
\hline H -1.4724 -1.9972 1.7712 & H - $1.4279-1.88811 .8068$ & H - $1.4454-1.93711 .8355$ & H -0.4280 $1.5765-2.3663$ \\
\hline H - $1.39181 .0168-2.1719$ & H - $1.38800 .8774-2.2371$ & H - $1.42490 .9353-2.2493$ & H - $1.8285-0.26232 .0987$ \\
\hline H $1.3476-1.97861 .2936$ & Н $1.2644-1.98481 .3044$ & H $1.3132-1.92771 .3311$ & Н $2.05700 .4243-1.6352$ \\
\hline H $2.2213-1.2802-0.6920$ & Н $2.1607-1.3569-0.7265$ & Н $2.1259-1.3490-0.7396$ & Н $2.5535-0.08370 .7159$ \\
\hline H $0.77321 .0680-2.9896$ & Н $0.87061 .0630-3.1092$ & Н $0.89451 .1038-3.0797$ & Н $0.7764-1.93443 .0948$ \\
\hline H $2.48820 .8691-2.6231$ & H $2.54940 .6341-2.6520$ & H $2.56290 .7335-2.6296$ & H $2.0278-0.68252 .6835$ \\
\hline H $1.4396-0.5544-2.7569$ & H $1.3137-0.6597-2.8571$ & H $1.3828-0.5699-2.8340$ & Н $0.2962-0.18892 .9352$ \\
\hline H $0.4001-0.62713 .1083$ & Н $0.4558-0.40843 .1092$ & Н $0.4970-0.36683 .1056$ & H $0.7889-0.9846-3.0948$ \\
\hline H $2.1265-0.28782 .9221$ & H $2.2066-0.18872 .8079$ & H $2.2150-0.12432 .7986$ & Н $2.1516-1.9170-2.3360$ \\
\hline Н 0.91820 .86692 .3370 & H 1.02701 .00372 .1594 & H 1.02721 .02682 .1701 & Н $0.4486-2.1523-1.7444$ \\
\hline H $4.92900 .8921-0.4419$ & H 4.62591 .38090 .2492 & H 4.77301 .12540 .1091 & H $3.8151-3.44980 .6157$ \\
\hline H 0.97132 .11980 .4319 & H 1.05692 .01820 .4258 & Н 0.83282 .09460 .3495 & Н $0.0795-2.7983-0.0114$ \\
\hline H $2.16972 .3524-0.6634$ & Н $2.16572 .2879-0.7594$ & H $2.19522 .2436-0.5418$ & H $1.0703-3.17751 .2036$ \\
\hline H -4.1042 -1.5233 1.7389 & H -3.7916 -2.1968 1.6482 & H -3.7707 -2.4833 1.4248 & H -2.3577 $2.8405-2.4789$ \\
\hline H -4.7881 -1.1925 0.1433 & H -4.6259 -0.7915 0.9060 & H -4.5231 -0.88811 .2107 & H -3.8151 2.0716-1.7128 \\
\hline H -3.9852 -2.7310 0.4569 & $\mathrm{H}-4.3273-2.2879-0.0637$ & H $-4.5107-2.0723-0.1236$ & H - $2.97083 .4498-0.8802$ \\
\hline
\end{tabular}

SSR- $a x$

\begin{tabular}{|c|c|c|c|}
\hline B3LYP/def2-TZVP & MM2 & MMFF9 & unoptimized \\
\hline C - $2.17600 .5025-0.5766$ & C - $2.06580 .4423-0.5899$ & C - $2.13250 .4448-0.6279$ & C -2.0829 1.07590 .4382 \\
\hline C - $2.0534-0.41640 .4678$ & C - $1.9507-0.40500 .4508$ & C - $-2.0314-0.42550 .4738$ & C - $1.69181 .5893-0.8087$ \\
\hline C $-0.8071-0.77730 .9747$ & C $-0.7550-0.68320 .9953$ & C $-0.7900-0.73111 .0358$ & C -0.4837 $1.2006-1.3915$ \\
\hline C $0.3284-0.21100 .4200$ & C $0.3381-0.10460 .4816$ & C $0.3501-0.16030 .4881$ & C $0.31990 .3083-0.7026$ \\
\hline C $0.22870 .6929-0.6319$ & C $0.24000 .7415-0.5506$ & C $0.25810 .7148-0.6051$ & C - $-0.0699-0.20340 .5401$ \\
\hline C - $1.01401 .0546-1.1326$ & C - $0.95581 .0183-1.0884$ & C - $0.97491 .0339-1.1575$ & C - 1.27140 .16641 .1199 \\
\hline C $1.7597-0.36680 .8921$ & C $1.7610-0.27600 .9456$ & C $1.7841-0.31820 .9422$ & C $1.6268-0.2794-1.1339$ \\
\hline C $2.57790 .2241-0.3235$ & C $2.53350 .2410-0.3005$ & C $2.54860 .1792-0.3287$ & C $2.1642-0.82150 .1985$ \\
\hline C $1.59161 .2105-1.0404$ & C $1.58721 .2796-0.9604$ & C $1.61971 .2438-0.9766$ & C $0.9405-1.18041 .0540$ \\
\hline C $1.79271 .3894-2.5432$ & C $1.77291 .4791-2.4680$ & C $1.82701 .5078-2.4629$ & C $1.2158-0.96962 .5370$ \\
\hline C $3.0656-0.8333-1.2773$ & C $2.8763-0.8874-1.2659$ & C $2.8886-0.9354-1.2998$ & C 2.99210 .23230 .8921 \\
\hline N $2.0478-1.73711 .2961$ & N $2.0579-1.68601 .2488$ & N $2.0873-1.67911 .3907$ & N $2.49860 .7230-1.6844$ \\
\hline O $4.3087-0.5667-1.7311$ & O $4.0831-0.7084-1.8397$ & O $3.9860-0.6497-2.0346$ & O $3.5579-0.03302 .0752$ \\
\hline O $2.4529-1.8129-1.6374$ & O $2.2007-1.8550-1.5214$ & O $2.3168-2.0000-1.4601$ & O 3.14401 .31540 .3793 \\
\hline H $4.5547-1.2670-2.3595$ & Н $4.2438-1.4485-2.4476$ & H $4.0900-1.4323-2.6170$ & C -3.3826 1.5076 1.0429 \\
\hline C -3.5386 0.8976 -1.0827 & C -3.4118 $0.7561-1.2001$ & C -3.4787 $0.7930-1.1931$ & C $1.5088-1.3269-2.2331$ \\
\hline H - $2.9505-0.85010 .8940$ & H - $2.8498-0.88270 .8737$ & H - $2.9268-0.87010 .9025$ & $\mathrm{H}-2.34352 .3048-1.3317$ \\
\hline H -0.7252 -1.4847 1.7899 & H -0.6714 - 1.37331 .8497 & H - $0.7182-1.40231 .8867$ & H - $0.17851 .5933-2.3727$ \\
\hline H - $1.09441 .7645-1.9487$ & H - $1.03221 .7180-1.9347$ & H - $1.04761 .7311-1.9868$ & H - $1.5790-0.24552 .0924$ \\
\hline C 1.98450 .54142 .1089 & C 2.03260 .58702 .1873 & C 2.05150 .61462 .1378 & H $2.8143-1.71020 .0364$ \\
\hline H 3.45270 .76580 .0319 & H 3.49460 .71590 .0092 & Н $3.50610 .6287-0.0314$ & Н $0.6262-2.24540 .9779$ \\
\hline Н $2.80811 .7228-2.7658$ & Н $2.82231 .7733-2.6978$ & Н $2.86291 .7991-2.6643$ & Н $1.9695-1.71142 .8840$ \\
\hline H $1.10442 .1415-2.9301$ & H $1.11652 .2916-2.8539$ & H $1.18572 .3285-2.8031$ & H $0.2721-1.10383 .1117$ \\
\hline H $1.60510 .4590-3.0822$ & H $1.53370 .5569-3.0436$ & H $1.58230 .6347-3.0772$ & H 1.60670 .05982 .6989 \\
\hline H $1.9652-2.36590 .5061$ & H $1.6277-2.29160 .5277$ & H $1.8597-2.32710 .6286$ & H $2.63101 .4700-1.0026$ \\
\hline H $2.9890-1.81931 .6643$ & Н $3.0736-1.87631 .2006$ & Н $3.0857-1.78301 .5552$ & Н $3.43010 .3286-1.8153$ \\
\hline H 3.01520 .45672 .4634 & H 3.09600 .49732 .5060 & Н 3.10510 .57822 .4392 & H 4.03550 .77352 .3325 \\
\hline H 1.78881 .58461 .8577 & H 1.82281 .66381 .9982 & H 1.81191 .65951 .9084 & H -3.8877 2.2288 0.3620 \\
\hline
\end{tabular}




\begin{tabular}{|c|c|c|c|}
\hline H 1.31170 .24812 .9149 & H 1.39690 .26533 .0436 & H 1.44490 .33533 .0085 & H -4.03550.6186 1.1914 \\
\hline H $1.74432 .1871-0.5698$ & H $1.71502 .2662-0.4491$ & H $1.75592 .1947-0.4436$ & H -3.1907 1.9963 2.0243 \\
\hline H - $4.32300 .3422-0.5691$ & H -4.2438 0.1959- -0.7181 & H - $4.2022-0.0078-1.0080$ & Н $2.1161-1.0136-3.1117$ \\
\hline H -3.7225 $1.9636-0.9189$ & H -3.6364 1.8425 -1.0994 & H -3.8493 $1.7152-0.7353$ & Н $1.8821-2.3048-1.8546$ \\
\hline H -3.6360 $0.7067-2.1546$ & H -3.4164 0.4936-2.2826 & H -3.4193 $0.9322-2.2777$ & Н $0.4427-1.4280-2.5363$ \\
\hline
\end{tabular}

RSR-eq

\begin{tabular}{|c|c|c|c|}
\hline B3LYP/def2-TZVP & MM2 & MMFF9 & unoptimized \\
\hline C 2.67380 .20841 .0688 & C 2.60170 .24131 .0742 & C 2.68130 .18651 .0599 & C $2.0975-1.6400-0.9184$ \\
\hline C 2.59141 .35670 .2753 & C 2.55131 .27150 .2078 & C 2.60721 .33270 .2456 & C $1.7064-2.15340 .3285$ \\
\hline C $1.39281 .7467-0.3154$ & C $1.41271 .5971-0.4259$ & C $1.40851 .7014-0.3699$ & C $0.4983-1.76470 .9113$ \\
\hline C $0.25640 .9829-0.0989$ & C $0.30870 .8782-0.1837$ & C $0.27800 .9255-0.1533$ & C $-0.3053-0.87240 .2224$ \\
\hline C $0.3114-0.14030 .7208$ & C $0.3462-0.15210 .6701$ & C $0.3438-0.21000 .6643$ & C $0.0845-0.3607-1.0203$ \\
\hline C $1.5112-0.53371 .2978$ & C $1.4841-0.47401 .3003$ & C $1.5317-0.58501 .2776$ & C $1.2860-0.7305-1.6001$ \\
\hline C - $1.10071 .1344-0.7633$ & C - $1.05601 .0719-0.7959$ & C - $1.11291 .1226-0.7221$ & C - $1.6122-0.28470 .6537$ \\
\hline C - 1.96980 .24540 .1896 & C - 1.91330 .24540 .2024 & C - 1.92770 .23010 .2666 & C - $2.14960 .2574-0.6787$ \\
\hline C - $1.0312-0.82720 .8325$ & C - $0.9885-0.84500 .8061$ & C - $0.9885-0.90920 .7490$ & C $-0.92590 .6163-1.5342$ \\
\hline C - $1.0438-2.21710 .1904$ & C - $-0.9541-2.20950 .1048$ & C $-0.9776-2.2070-0.0552$ & C - $0.48222 .0538-1.2972$ \\
\hline C $-3.2827-0.2693-0.3267$ & C -3.3219-0.1024-0.2340 & C -3.2906 -0.1981-0.2105 & C - $2.99331 .4851-0.4377$ \\
\hline $\mathrm{N}-1.53872 .5306-0.8233$ & $\mathrm{~N}-1.46532 .4876-0.7756$ & $\mathrm{~N}-1.51652 .5338-0.6518$ & $\mathrm{~N}-2.4841-1.28701 .2042$ \\
\hline O -3.9633 -0.9117 0.6471 & O -3.8470 - 1.11830 .4763 & O -3.8311-1.1782 0.5405 & O -3.5617 2.1193-1.4697 \\
\hline O -3.7416 - $0.1282-1.4355$ & O -3.9743 $0.4762-1.0688$ & O -3.9641 0.3313-1.0786 & O -3.1556 1.89530 .6869 \\
\hline H $-4.8076-1.21480 .2730$ & H - $4.7688-1.24100 .1960$ & H -4.7334 - 1.29340 .1731 & C $3.3971-2.0717-1.5230$ \\
\hline C $3.9905-0.21561 .6629$ & C $3.8856-0.12551 .7809$ & C $3.9709-0.17161 .7394$ & C - 1.49430 .76281 .7530 \\
\hline Н 3.48871 .93890 .1016 & Н 3.45841 .86530 .0069 & H 3.49171 .94600 .0853 & Н $2.3580-2.86890 .8515$ \\
\hline H $1.34992 .6251-0.9469$ & H $1.38402 .4393-1.1352$ & H $1.36012 .5834-1.0026$ & Н $0.1931-2.15741 .8925$ \\
\hline H $1.5530-1.42261 .9188$ & H $1.4967-1.32332 .0014$ & H $1.5669-1.46431 .9150$ & H $1.5935-0.3186-2.5726$ \\
\hline C - $0.97690 .6428-2.2154$ & C - $1.05730 .5756-2.2498$ & C - $1.13210 .7005-2.1976$ & H - $2.7885-0.4972-1.1898$ \\
\hline H - 2.25160 .90731 .0148 & H -2.1161 0.94501 .0589 & H -2.1388 0.8415 1.1619 & H - $1.10220 .5568-2.6315$ \\
\hline H - $2.0204-2.68970 .3042$ & H - $1.9122-2.76290 .2067$ & H - $1.9430-2.7182-0.0133$ & H - $1.25542 .7510-1.6908$ \\
\hline H - $0.3090-2.85510 .6839$ & H $-0.1690-2.86110 .5522$ & H - $0.2328-2.90110 .3522$ & Н $0.48212 .2353-1.8226$ \\
\hline H - $0.7916-2.1799-0.8707$ & H - $0.7284-2.1261-0.9789$ & H $-0.7222-2.0539-1.1057$ & H -0.3491 2.2257-0.2056 \\
\hline H -1.6935 2.9011 0.1086 & $\mathrm{H}-1.42362 .86110 .1882$ & H - 1.47292 .85600 .3136 & $\mathrm{H}-2.6165-2.03410 .5224$ \\
\hline H -2.4181 2.5966 -1.3251 & H -2.4541 2.5778 -1.0684 & H -2.4915 2.6149-0.9441 & H -3.4156 -0.8927 1.3351 \\
\hline H - $1.91850 .7514-2.7488$ & H - $2.05160 .6941-2.7325$ & H - $2.11880 .8305-2.6532$ & H - $4.05002 .8689-1.0895$ \\
\hline H 4.82080 .30711 .1883 & H 4.76880 .41291 .3703 & H 4.83000 .13761 .1349 & H $3.9023-2.7929-0.8422$ \\
\hline H $4.1485-1.28941 .5365$ & H $4.0910-1.21581 .6831$ & H $4.0486-1.25431 .8838$ & H $4.0500-1.1827-1.6716$ \\
\hline H 4.03040 .00132 .7348 & Н 3.80830 .12292 .8639 & H 4.02840 .32092 .7149 & Н $3.2053-2.5604-2.5045$ \\
\hline H - $0.6704-0.4017-2.2608$ & H - $0.7667-0.4921-2.3374$ & H - $-0.8376-0.3417-2.3403$ & H -2.1015 0.4495 2.6315 \\
\hline H -0.2149 $1.2427-2.7137$ & H -0.3348 $1.1602-2.8639$ & H -0.4303 $1.3029-2.7891$ & H - 1.86761 .74071 .3744 \\
\hline H - $-1.3074-0.95141 .8817$ & H - $1.2272-1.00261 .8864$ & H - $1.2173-1.14041 .7968$ & H - 0.42820 .86392 .0561 \\
\hline
\end{tabular}

RSR-ax

\begin{tabular}{|c|c|c|c|}
\hline B3LYP/def2-TZVP & MM2 & MMFF9 & unoptimized \\
\hline C 2.29490 .34490 .2319 & C $2.1895-0.25580 .2771$ & C $2.2771-0.29690 .2836$ & C $2.3179-0.9230-0.2337$ \\
\hline C 2.24240 .59550 .8049 & C $2.11780 .6257-0.7386$ & C $2.19940 .6506-0.7552$ & C $1.9268-1.43641 .0132$ \\
\hline C 0.99500 .96191 .3137 & C $0.91660 .8976-1.2816$ & C $0.95450 .9655-1.3164$ & C $0.7187-1.04771 .5960$ \\
\hline C 0.16260 .39630 .7909 & C $-0.18090 .2939-0.8048$ & C - $-0.18550 .3574-0.8090$ & C $-0.0849-0.15540 .9071$ \\
\hline C 0.09310 .53380 .2371 & C - $-0.0957-0.57700 .2091$ & C $-0.1017-0.57840 .2361$ & C $0.30490 .3563-0.3356$ \\
\hline C 1.13780 .91440 .7539 & C $1.0925-0.86420 .7564$ & C $1.1284-0.91740 .7804$ & C $1.5064-0.0135-0.9154$ \\
\hline C 1.58940 .60891 .2437 & C - $1.60440 .4674-1.2689$ & C - $1.61180 .5098-1.2706$ & C - 1.39180 .43231 .3384 \\
\hline C 2.42930 .01980 .0935 & C - $2.3714-0.0537-0.0252$ & C - $2.3870-0.0397-0.0442$ & C - 1.92920 .97440 .0060 \\
\hline C 1.46081 .08380 .5956 & C - $1.4430-1.14550 .5773$ & C - $1.4681-1.13140 .5850$ & C $-0.70551 .3333-0.8495$ \\
\hline C 1.68211 .24832 .0968 & C - $1.6537-1.39892 .0753$ & C - $1.6866-1.37232 .0819$ & C - $0.98081 .1226-2.3325$ \\
\hline C 2.94140 .98430 .9055 & C - 2.65551 .06460 .9718 & C - -2.73961 .04940 .9515 & C - $-2.7571-0.0793-0.6876$ \\
\hline
\end{tabular}




\begin{tabular}{|c|c|c|c|}
\hline C 1.93272 .03751 .6628 & C - $1.98681 .8735-1.7405$ & C -2.0130 1.9021-1.7405 & C - $-2.3152-0.62931 .9215$ \\
\hline O 4.20230 .68911 .2983 & O -3.9677 1.1498 1.2680 & $\mathrm{O}-4.05721 .05671 .2470$ & O -3.3229 0.1860-1.8707 \\
\hline O 2.34091 .93231 .3563 & $\mathrm{O}-1.85361 .81551 .4741$ & O - 1.99851 .85981 .4784 & O - $2.9091-1.1625-0.1747$ \\
\hline N 1.58872 .41610 .0120 & $\mathrm{~N}-1.6347-2.4093-0.1591$ & $\mathrm{~N}-1.6386-2.4302-0.0933$ & $\mathrm{~N}-0.29942 .7093-0.7511$ \\
\hline H 3.25840 .63730 .6341 & H $3.1702-0.48810 .7251$ & Н $3.2444-0.56040 .7069$ & C $2.8136-2.41001 .7250$ \\
\hline C 3.51021 .19121 .3613 & C $3.37551 .2883-1.2502$ & C $3.45651 .2768-1.2851$ & Н $3.2729-1.2402-0.6780$ \\
\hline H 0.93811 .68642 .1173 & Н $0.82911 .6110-2.1149$ & Н $0.88441 .6752-2.1356$ & Н $0.4135-1.44042 .5772$ \\
\hline H 1.20381 .64811 .5475 & Н $1.1711-1.58811 .5821$ & H $1.2054-1.65891 .5694$ & Н $1.81400 .3984-1.8879$ \\
\hline H 1.75710 .02722 .1205 & $\mathrm{H}-1.7603-0.2463-2.1165$ & H - $1.7556-0.1838-2.1105$ & H -1.3056 1.1979 2.1418 \\
\hline H 3.29490 .54880 .4863 & H -3.3493 - $0.4940-0.3348$ & H -3.3357 -0.4759-0.3880 & H -2.5794 1.86310 .1681 \\
\hline Н 0.97551 .98092 .4853 & H - $1.0275-2.24682 .4339$ & H -1.0643 -2.1971 2.4513 & H - $0.79102 .0705-2.8840$ \\
\hline H 2.69101 .61362 .3027 & H - $2.7162-1.66422 .2788$ & H - $2.7310-1.63012 .2930$ & H - $2.04230 .8186-2.4727$ \\
\hline H 1.53320 .31032 .6293 & H - $1.3912-0.51442 .6967$ & H - $1.4219-0.49702 .6850$ & $\mathrm{H}-0.31070 .3250-2.7243$ \\
\hline H 1.34132 .31982 .5352 & $\mathrm{H}-1.47732 .1312-2.6967$ & H - $1.50712 .1506-2.6802$ & H - $1.8956-0.99862 .8840$ \\
\hline H 2.98612 .12411 .9396 & H -3.0838 1.9371-1.9239 & H -3.0915 $1.9530-1.9229$ & H -3.3208 -0.1874 2.1010 \\
\hline H 1.72432 .74660 .8641 & H - $1.71382 .6581-1.0009$ & H -1.7445 2.6814-1.0203 & H - $2.4038-1.47721 .2060$ \\
\hline H 4.46761 .34241 .9674 & H -4.0880 1.87021 .9080 & H -4.1477 1.79121 .8915 & $\mathrm{H}-3.8006-0.6205-2.1280$ \\
\hline H 1.40162 .38001 .0086 & H - $1.3224-2.2924-1.1386$ & H - $1.3997-2.3386-1.0803$ & H - 0.09402 .93440 .2223 \\
\hline H 2.53182 .77270 .1086 & H - $2.6373-2.6581-0.2147$ & H - $2.6137-2.7204-0.0504$ & H - $1.08483 .3137-0.9928$ \\
\hline H 3.29541 .92092 .1418 & Н $3.17732 .0027-2.0802$ & H $3.23542 .1547-1.9011$ & Н $2.3432-2.70442 .6898$ \\
\hline H 4.15090 .41801 .7929 & H $4.08800 .5216-1.6312$ & H $4.00560 .5544-1.8969$ & Н $3.8006-1.93551 .9236$ \\
\hline H 4.08671 .69350 .5808 & Н $3.87291 .8572-0.4318$ & H $4.09521 .6082-0.4599$ & Н $2.9562-3.31371 .0911$ \\
\hline
\end{tabular}

\begin{tabular}{|c|c|c|c|}
\hline B3LYP/def2-TZVP & MM2 & MMFF9 & unoptimized \\
\hline C 2.57580 .44070 .9448 & C $2.5053-0.43000 .9184$ & C $2.5665-0.44200 .9541$ & C $2.0975-1.6400-0.4382$ \\
\hline C 2.54650 .40870 .1627 & C $2.48320 .4040-0.1391$ & C $2.55680 .4203-0.1585$ & C $1.7064-2.15340 .8087$ \\
\hline C 1.35340 .70400 .8187 & C $1.34030 .6754-0.7900$ & C $1.36650 .7169-0.8260$ & C $0.4983-1.76471 .3915$ \\
\hline C 0.18030 .13680 .3537 & C $0.20560 .1047-0.3659$ & C $0.18530 .1460-0.3723$ & C - $0.3053-0.87240 .7026$ \\
\hline C 0.18790 .72110 .7440 & C $0.2108-0.72840 .6821$ & C $0.1865-0.71840 .7310$ & C $0.0845-0.3607-0.5401$ \\
\hline C 1.37761 .00981 .3960 & C $1.3550-0.99871 .3255$ & C $1.3694-1.03031 .3880$ & C $1.2860-0.7305-1.1199$ \\
\hline C 1.20760 .29390 .9371 & C - $1.17000 .2605-0.9610$ & C - $1.20400 .2886-0.9557$ & C - $1.6122-0.28471 .1339$ \\
\hline C 2.07860 .20070 .2921 & C - $2.0390-0.22980 .2254$ & C - $2.0575-0.17880 .2610$ & C - $-2.14960 .2574-0.1985$ \\
\hline C 1.20851 .23371 .0366 & C - $1.1691-1.26050 .9865$ & C - $1.2011-1.24110 .9995$ & C $-0.92590 .6163-1.0540$ \\
\hline C 1.54511 .39442 .5153 & C - $1.4973-1.34582 .4812$ & C - $1.5472-1.33822 .4826$ & C - $1.20120 .4055-2.5370$ \\
\hline C 3.45910 .59500 .1426 & C $-3.4473-0.6140-0.1822$ & C -3.4332 - $0.6321-0.1535$ & C - 2.99331 .48510 .0425 \\
\hline N 1.46391 .66291 .3600 & $\mathrm{~N}-1.47561 .6638-1.2844$ & $\mathrm{~N}-1.48871 .6748-1.3399$ & $\mathrm{~N}-2.4841-1.28701 .6844$ \\
\hline O 3.69311 .91950 .1231 & O -3.7539-1.8926 0.1024 & $\mathrm{O}-3.7446-1.89650 .1752$ & O -3.5617 2.1193-0.9895 \\
\hline O 4.29250 .19900 .5252 & $\mathrm{O}-4.23610 .1239-0.7222$ & $\mathrm{O}-4.25220 .0617-0.7382$ & O -3.1556 1.89531 .1670 \\
\hline C 1.36510 .62652 .1541 & C - $1.3094-0.6132-2.2164$ & C - $1.3290-0.6070-2.1985$ & C - 1.49430 .76282 .2331 \\
\hline C 3.87900 .74411 .6342 & C $3.7924-0.73701 .6477$ & C $3.8615-0.78441 .6311$ & C $3.3971-2.0717-1.0429$ \\
\hline H 3.47250 .84950 .5126 & H $3.41700 .8746-0.4886$ & Н $3.48480 .8619-0.5150$ & Н $2.3580-2.86891 .3317$ \\
\hline H 1.34101 .37161 .6706 & H $1.33281 .3516-1.6595$ & H $1.36551 .3777-1.6882$ & Н $0.1931-2.15742 .3727$ \\
\hline H 1.38781 .66762 .2580 & H $1.3554-1.68732 .1843$ & H $1.3717-1.72252 .2248$ & H $1.5935-0.3186-2.0924$ \\
\hline H 2.20400 .67490 .9334 & $\mathrm{H}-2.17360 .64210 .9160$ & H - 2.21290 .66490 .9530 & H - $2.7885-0.4972-0.7096$ \\
\hline H 1.32612 .20720 .5513 & $\mathrm{H}-1.2440-2.26920 .5144$ & H - $1.2946-2.22990 .5330$ & H -0.6116 1.6813 -0.9779 \\
\hline H 0.88102 .11802 .9900 & H - $0.8713-2.11062 .9937$ & H -0.9274 -2.0911 2.9811 & $\mathrm{H}-1.95501 .1473-2.8840$ \\
\hline H 2.56841 .75382 .6456 & H -2.5610 -1.6344 2.6388 & H -2.5935 -1.6255 2.6251 & H - $0.25760 .5397-3.1117$ \\
\hline H 1.43870 .44363 .0424 & H - $1.3302-0.37002 .9911$ & H - $1.3849-0.38482 .9982$ & H - $1.5921-0.6239-2.6989$ \\
\hline H 1.35312 .30120 .5791 & H - $1.26372 .2692-0.4728$ & H - $1.34962 .2845-0.5347$ & H -2.6165 -2.0341 1.0026 \\
\hline H 2.41921 .75551 .6904 & H -2.4890 1.7827 - 1.4596 & H -2.4780 $1.7554-1.5849$ & H -3.4156 -0.8927 1.8153 \\
\hline H 4.59102 .06860 .4649 & H -4.6615 - $2.0632-0.1981$ & H -4.6590 -2.0169-0.1599 & $\mathrm{H}-4.05002 .8689-0.6094$ \\
\hline Н 2.35870 .52412 .5967 & H - $2.3210-0.5165-2.6704$ & H -2.3234 - $0.5369-2.6533$ & H -2.1015 0.4495 3.1117 \\
\hline H 1.21181 .67041 .8784 & H - $1.1386-1.6896-1.9888$ & H -1.1474 -1.6615 -1.9599 & H - 1.86761 .74071 .8546 \\
\hline Н 0.62610 .35662 .9080 & H - $0.5714-0.3102-2.9937$ & H - $-0.6007-0.3238-2.9689$ & Н - 0.42820 .86392 .5363 \\
\hline
\end{tabular}




\begin{tabular}{llll}
\hline H 4.6978 0.1731 1.1976 & H 4.6615 -0.1720 1.2428 & H 4.5882 0.0275 1.5220 & H 3.9023 -2.7929 -0.3620 \\
H 4.1277 1.8057 1.5487 & H 4.0304 -1.8222 1.5675 & H 4.2822 - 1.69551 .1946 & H 4.0500 - $1.1827-1.1914$ \\
H 3.8295 0.5018 2.6992 & H 3.6985 -0.4751 2.7262 & H 3.7091 -0.9413 2.7042 & H 3.2053 -2.5604-2.0243 \\
\hline
\end{tabular}

RSS-ax

\begin{tabular}{|c|c|c|c|}
\hline B3LYP/def2-TZVP & MM2 & MMFF9 & unoptimized \\
\hline C 2.38600 .12200 .5755 & C 2.26980 .00200 .6197 & C $2.3648-0.04650 .6241$ & C $2.3179-0.9230-0.4445$ \\
\hline C 2.34360 .81990 .4628 & C $2.20880 .8808-0.3991$ & C $2.29660 .8838-0.4301$ & C $1.9268-1.43640 .8024$ \\
\hline C 1.10111 .21550 .9562 & C $1.00751 .1818-0.9258$ & C $1.05071 .2272-0.9703$ & C $0.7187-1.04771 .3852$ \\
\hline C 0.06580 .67800 .4186 & C - $-0.09750 .6089-0.4295$ & C $-0.09520 .6421-0.4464$ & C $-0.0849-0.15540 .6963$ \\
\hline C 0.00550 .25050 .6074 & C - $-0.0259-0.25750 .5876$ & C - $-0.0244-0.27300 .6117$ & C $0.30490 .3563-0.5464$ \\
\hline C 1.22380 .66111 .1115 & C $1.1632-0.57381 .1174$ & C $1.2068-0.63131 .1442$ & C $1.5064-0.0135-1.1262$ \\
\hline C 1.48540 .94950 .8522 & C - $1.51110 .8331-0.8954$ & C - $1.51250 .8729-0.8914$ & C - 1.39180 .43231 .1276 \\
\hline C 2.34870 .23000 .2176 & C - 2.31240 .24590 .2967 & C - -2.32140 .23110 .2688 & C - $1.92920 .9744-0.2048$ \\
\hline C 1.37120 .75241 .0250 & C - $1.3784-0.79570 .9781$ & C - $1.3920-0.79670 .9854$ & C $-0.70551 .3333-1.0603$ \\
\hline C 1.55660 .71082 .5409 & C - $1.5852-0.93422 .4928$ & C - $1.6037-0.91542 .4994$ & C - $-0.98081 .1226-2.5433$ \\
\hline C 3.04631 .20041 .1316 & C - 2.70891 .36611 .2542 & C - -2.80631 .32401 .2006 & C - $2.7571-0.0793-0.8985$ \\
\hline C 1.76050 .45372 .2768 & C - $-1.78620 .1237-2.2275$ & C - $-1.80320 .2364-2.2476$ & C - -1.19261 .54892 .1441 \\
\hline O 4.17840 .67381 .6483 & O -3.9748 1.2236 1.6954 & O -4.0254 1.05041 .7126 & O -3.3229 0.1860-2.0816 \\
\hline O 2.67012 .31381 .4183 & O -2.0230 2.2941 1.6111 & O -2.2370 2.3558 1.5099 & O - $2.9091-1.1625-0.3856$ \\
\hline N 1.50612 .15230 .6077 & $\mathrm{~N}-1.5520-2.11780 .3476$ & $\mathrm{~N}-1.5489-2.15090 .4239$ & $\mathrm{~N}-0.29942 .7093-0.9620$ \\
\hline C 3.62051 .38161 .0323 & C $3.47591 .5067-0.9324$ & C $3.55921 .4954-0.9621$ & C $2.8136-2.41001 .5141$ \\
\hline Н 3.34710 .43710 .9663 & H $3.2500-0.25611 .0544$ & H $3.3300-0.32351 .0422$ & Н $3.2729-1.2402-0.8888$ \\
\hline H 1.04911 .94091 .7604 & H $0.91711 .8941-1.7605$ & Н $0.97601 .9425-1.7840$ & Н $0.4135-1.44042 .3663$ \\
\hline Н 1.27901 .39481 .9060 & H $1.2351-1.29771 .9438$ & H $1.2769-1.36291 .9430$ & H $1.81400 .3984-2.0987$ \\
\hline H 1.68802 .02150 .8173 & H - $1.71211 .9234-1.0243$ & H - $1.70381 .9506-0.9645$ & H - $-2.0715-0.28821 .6352$ \\
\hline H 3.12100 .38340 .2421 & H -3.2533 -0.2308 - 0.0656 & H -3.2254 - $0.2500-0.1301$ & H - $2.57941 .8631-0.0428$ \\
\hline Н 0.80621 .34543 .0100 & H - $0.9429-1.73732 .9192$ & H - $0.9536-1.68212 .9392$ & H - $0.79102 .0705-3.0948$ \\
\hline Н 2.54151 .08472 .8263 & H -2.6409-1.2067 2.7201 & H - $2.6373-1.19252 .7367$ & H - $2.04230 .8186-2.6835$ \\
\hline H 1.43900 .30072 .9291 & H -1.3389 0.00323 .0395 & H - 1.37050 .02023 .0205 & $\mathrm{H}-0.31070 .3250-2.9352$ \\
\hline H 1.09480 .94582 .9875 & H - $1.14420 .5355-3.0395$ & H -1.1994 $0.7043-3.0331$ & H - 0.80351 .12073 .0948 \\
\hline Н 2.78970 .67022 .5719 & H - $2.84740 .2623-2.5366$ & $\mathrm{H}-2.85700 .3669-2.5146$ & H - 2.16612 .05312 .3360 \\
\hline H 1.59370 .62342 .3580 & H - $1.5907-0.9696-2.1674$ & $\mathrm{H}-1.5797-0.8346-2.2631$ & $\mathrm{H}-0.46312 .28841 .7444$ \\
\hline H 4.56091 .32352 .2616 & H -4.1680 1.9548 2.3048 & H -4.2149 1.8190 2.2926 & H -3.8006 - $0.6205-2.3388$ \\
\hline H 1.35712 .24740 .3910 & $\mathrm{H}-1.0421-2.1417-0.5520$ & H - $1.2799-2.1520-0.5579$ & $\mathrm{H}-0.09402 .93440 .0114$ \\
\hline H 2.44002 .49460 .8111 & H - $2.5432-2.29410 .1103$ & H - $2.5256-2.43380 .4658$ & H - $1.08483 .3137-1.2036$ \\
\hline Н 3.41772 .10741 .8195 & Н $3.28542 .2113-1.7725$ & Н $3.35502 .1860-1.7869$ & Н $2.3432-2.70442 .4789$ \\
\hline H 4.24120 .58911 .4581 & H $4.16800 .7185-1.3073$ & H $4.22800 .7139-1.3370$ & H $3.8006-1.93551 .7128$ \\
\hline Н 4.21191 .87890 .2597 & Н $3.99302 .0774-0.1277$ & H $4.06782 .0568-0.1717$ & H $2.9562-3.31370 .8802$ \\
\hline
\end{tabular}

\section{RDC Data $\beta$-aa-H*}

B3LYP/def2-TZVP

\begin{tabular}{|c|c|c|c|c|c|c|c|c|c|}
\hline \multirow{2}{*}{ Coupling } & \multirow{2}{*}{$D_{\text {Exp }} / \mathrm{Hz}$} & \multicolumn{2}{|c|}{$D_{\text {Calc }}(R S-e q) / \mathrm{Hz}$} & \multicolumn{2}{|c|}{$D_{\text {Calc }}(R S-a x) / \mathrm{Hz}$} & \multicolumn{2}{|c|}{$D_{\text {Calc }}(S S-e q) / \mathrm{Hz}$} & \multicolumn{2}{|c|}{$D_{\text {Calc }}(S S-a x) / \mathrm{Hz}$} \\
\hline & & $(-i)$ & $(-i i)$ & $(-i)$ & $(-i i)$ & $(-i)$ & $(-i i)$ & $(-i)$ & $(-i i)$ \\
\hline $1-\mathrm{CH}_{3}$ & -6.5 & -0.1 & -0.1 & 1.3 & -0.5 & -7.9 & -6.2 & -5.1 & -5.8 \\
\hline $\mathrm{C} 2-\mathrm{H} 2$ & 16.1 & 15.6 & 15.6 & 15.5 & 15.6 & 16.0 & 16.2 & 15.9 & 15.9 \\
\hline C3-H3(I) & 4.5 & 5.2 & 5.1 & 5.4 & 4.3 & 4.6 & 4.4 & 5.1 & 5.2 \\
\hline C3-H3(II) & 10.2 & 10.9 & 10.9 & 10.0 & 10.9 & 10.2 & 10.1 & 10.8 & 10.7 \\
\hline C4-H4 & 12.7 & 12.1 & 12.0 & 13.3 & 13.1 & 12.6 & 12.3 & 12.3 & 12.4 \\
\hline $5-\mathrm{CH}_{3}$ & 6.6 & 6.5 & 6.6 & 0.0 & 0.6 & 6.1 & 5.8 & 0.8 & 0.2 \\
\hline C6-H6 & -11.4 & -11.9 & -11.7 & -11.2 & -11.0 & -11.4 & -11.3 & -11.1 & -11.4 \\
\hline$Q$ factor & & 0.25 & 0.22 & 0.37 & 0.31 & 0.06 & 0.04 & 0.24 & 0.22 \\
\hline
\end{tabular}


MM2

\begin{tabular}{ccccccccccc}
\multirow{2}{*}{ Coupling } & $\begin{array}{c}D_{\operatorname{Exp}} \\
\text { /Hz }\end{array}$ & \multicolumn{2}{c}{$D_{\text {Calc }}(R S-e q) / \mathrm{Hz}$} & \multicolumn{2}{c}{$D_{\text {Calc }}(R S-a x) / \mathrm{Hz}$} & \multicolumn{2}{c}{$D_{\text {Calc }(R S-e q) / H z}$} & \multicolumn{2}{c}{$D_{\text {Calc }}(R S-a x) / \mathrm{Hz}$} \\
& $(-i)$ & $(-i i)$ & $(-i)$ & $(-i i)$ & $(-i)$ & $(-i i)$ & $(-i)$ & $(-i i)$ \\
\hline 1-CH3 & -6.5 & 1.0 & 1.1 & 1.3 & -0.4 & -7.9 & -6.7 & -5.6 & -5.3 \\
C2-H2 & 16.1 & 14.9 & 14.8 & 15.3 & 15.4 & 15.9 & 16.1 & 15.7 & 15.8 \\
C3-H3(I) & 4.5 & 5.6 & 5.6 & 5.4 & 4.2 & 4.6 & 4.4 & 5.3 & 4.9 \\
C3-H3(II) & 10.2 & 11.4 & 11.3 & 9.9 & 11.0 & 10.2 & 10.2 & 10.6 & 11.0 \\
C4-H4 & 12.7 & 11.7 & 11.6 & 13.4 & 13.1 & 12.4 & 12.7 & 12.6 & 12.7 \\
5-CH3 & 6.6 & 7.6 & 7.6 & -2.0 & -1.1 & 7.1 & 7.0 & -1.9 & -1.2 \\
C6-H6 & -11.4 & -12.0 & -11.9 & -10.8 & -10.8 & -11.4 & -11.5 & -10.7 & -10.9 \\
Q factor & & 0.29 & 0.29 & 0.43 & 0.36 & 0.06 & 0.03 & 0.32 & 0.27
\end{tabular}

MMFF9

\begin{tabular}{|c|c|c|c|c|c|c|c|c|c|}
\hline \multirow{2}{*}{ Coupling } & \multirow{2}{*}{$D_{\text {Exp }} / \mathrm{Hz}$} & \multicolumn{2}{|c|}{$D_{\text {Calc }}(R S-e q) / \mathrm{Hz}$} & \multicolumn{2}{|c|}{$D_{\text {Calc }}(R S-a x) / \mathrm{Hz}$} & \multicolumn{2}{|c|}{$D_{\text {Calc }}(S S-e q) / \mathrm{Hz}$} & \multicolumn{2}{|c|}{$D_{\text {Calc }}(S S-a x) / \mathrm{Hz}$} \\
\hline & & $(-i)$ & $(-i i)$ & $(-i)$ & $(-i i)$ & $(-i)$ & $(-i i)$ & $(-i)$ & $(-i i)$ \\
\hline $1-\mathrm{CH}_{3}$ & -6.5 & 0.8 & 0.8 & 1.5 & -0.2 & -8.3 & -7.4 & -4.9 & -4.7 \\
\hline $\mathrm{C} 2-\mathrm{H} 2$ & 16.1 & 15.2 & 15.2 & 15.5 & 15.6 & 15.8 & 16.0 & 15.8 & 15.9 \\
\hline C3-H3(I) & 4.5 & 5.5 & 5.4 & 5.4 & 4.3 & 4.6 & 4.5 & 5.3 & 5.0 \\
\hline C3-H3(II) & 10.2 & 11.1 & 11.2 & 9.9 & 11.0 & 10.3 & 10.3 & 10.7 & 11.0 \\
\hline $\mathrm{C} 4-\mathrm{H} 4$ & 12.7 & 11.9 & 11.8 & 13.4 & 13.2 & 12.4 & 12.6 & 12.7 & 12.8 \\
\hline $5-\mathrm{CH}_{3}$ & 6.6 & 6.9 & 6.6 & -0.4 & 0.5 & 6.8 & 6.6 & -0.8 & 0.0 \\
\hline C6-H6 & -11.4 & -11.9 & -11.8 & -11.0 & -10.9 & -11.4 & -11.5 & -10.8 & -11.0 \\
\hline$Q$ factor & & 0.28 & 0.27 & 0.39 & 0.32 & 0.07 & 0.04 & 0.28 & 0.25 \\
\hline
\end{tabular}

Unoptimized

\begin{tabular}{cccccccccc}
\multirow{2}{*}{ Coupling } & \multicolumn{2}{c}{$D_{\text {Exp }} / \mathrm{Hz}$} & \multicolumn{2}{c}{$D_{\text {Calc }}(R S-e q) / \mathrm{Hz}$} & \multicolumn{2}{c}{$D_{\text {Calc }(R S-a x) / \mathrm{Hz}}$} & \multicolumn{2}{c}{$D_{\text {Calc }}(S S-e q) / \mathrm{Hz}$} & \multicolumn{2}{c}{$D_{\text {Calc }}(S S-a x) / \mathrm{Hz}$} \\
& & $(-i)$ & $(-i i)$ & $(-i)$ & $(-i)$ & $(-i)$ & $(-i i)$ & $(-i)$ & $(-i i)$ \\
\hline 1-CH & -6.5 & 0.8 & 0.2 & 1.9 & 0.1 & -7.5 & -6.1 & -7.7 & -6.8 \\
C2-H2 & 16.1 & 15.4 & 15.5 & 15.3 & 15.4 & 16.0 & 16.2 & 15.6 & 15.8 \\
C3-H3(I) & 4.5 & 5.4 & 5.1 & 5.4 & 4.3 & 4.5 & 4.4 & 5.1 & 4.9 \\
C3-H3(II) & 10.2 & 10.9 & 11.1 & 9.9 & 11.0 & 10.2 & 10.1 & 10.6 & 10.9 \\
C4-H4 & 12.7 & 11.9 & 11.8 & 13.3 & 13.1 & 12.5 & 12.8 & 12.7 & 12.8 \\
5-CH3 & 6.6 & 6.5 & 6.2 & -1.0 & -0.5 & 6.7 & 6.5 & -1.0 & -0.5 \\
C6-H6 & -11.4 & -12.0 & -11.8 & -11.1 & -10.9 & -11.3 & -11.5 & -10.7 & -10.9 \\
Q factor & & 0.27 & 0.25 & 0.42 & 0.36 & 0.05 & 0.03 & 0.28 & 0.26
\end{tabular}




\section{RDC Data $\beta$-aa-Me*}

B3LYP/def2-TZVP

\begin{tabular}{|c|c|c|c|c|c|}
\hline Coupling & $D_{\operatorname{Exp}} / \mathrm{Hz}$ & $D_{\text {Calc }}(S S R-e q) / \mathrm{Hz}$ & $D_{\text {Calc }}(S S R-a x / \mathrm{Hz}$ & $D_{\text {Calc }}(S S S-e q) / \mathrm{Hz}$ & $D_{\text {Calc }}(S S S-a x) / \mathrm{Hz}$ \\
\hline $1-\mathrm{CH}_{3}$ & -8.9 & 1.4 & -8.3 & -0.0 & -4.2 \\
\hline $\mathrm{C} 2-\mathrm{H} 2$ & 1.1 & 4.3 & 1.1 & 2.6 & 1.3 \\
\hline $\mathrm{C} 3-\mathrm{H} 3$ & 13.5 & 11.5 & 13.3 & 13.4 & 13.7 \\
\hline $3-\mathrm{CH}_{3}$ & -8.8 & 1.4 & -8.3 & 0.6 & -7.2 \\
\hline $\mathrm{C} 4-\mathrm{H} 4$ & -4.1 & -1.5 & -4.1 & -2.4 & -4.2 \\
\hline $5-\mathrm{CH}_{3}$ & 10.2 & 4.9 & 11.8 & 5.3 & 12.7 \\
\hline C6-H6 & 12.0 & 12.9 & 11.8 & 12.5 & 11.6 \\
\hline$Q$ factor & & 0.66 & 0.08 & 0.57 & 0.23 \\
\hline Coupling & $D_{\operatorname{Exp}} / \mathrm{Hz}$ & $D_{\text {Calc }}(R S R-e q) / \mathrm{Hz}$ & $D_{\text {Calc }}(R S R-a x) / \mathrm{Hz}$ & $D_{\text {Calc }}(R S S-e q) / \mathrm{Hz}$ & $D_{\text {Calc }}(R S S-a x) / \mathrm{Hz}$ \\
\hline $1-\mathrm{CH}_{3}$ & -8.9 & -11.1 & 0.8 & -11.1 & -2.8 \\
\hline $\mathrm{C} 2-\mathrm{H} 2$ & 1.1 & 1.3 & 0.5 & 2.4 & 0.8 \\
\hline $\mathrm{C} 3-\mathrm{H} 3$ & 13.5 & 13.2 & 14.0 & 13.0 & 12.8 \\
\hline $3-\mathrm{CH}_{3}$ & -8.8 & -7.0 & -1.1 & -6.5 & -8.9 \\
\hline $\mathrm{C} 4-\mathrm{H} 4$ & -4.1 & -3.8 & -3.1 & -3.1 & -4.0 \\
\hline $5-\mathrm{CH}_{3}$ & 10.2 & 9.4 & 8.0 & 3.7 & 14.1 \\
\hline C6-H6 & 12.0 & 12.1 & 12.0 & 12.8 & 11.5 \\
\hline$Q$ factor & & 0.13 & 0.52 & 0.31 & 0.30 \\
\hline
\end{tabular}

MM2

\begin{tabular}{|c|c|c|c|c|c|}
\hline Coupling & $D_{\operatorname{Exp}} / \mathrm{Hz}$ & $D_{\text {Calc }}(S S R-e q) / \mathrm{Hz}$ & $D_{\text {Calc }}(S S R-a x / H z$ & $D_{\text {Calc }}(S S S-e q) / \mathrm{Hz}$ & $D_{\text {Calc }}(S S S-a x) / \mathrm{Hz}$ \\
\hline $1-\mathrm{CH}_{3}$ & -8.9 & 3.4 & -9.1 & 0.1 & -5.1 \\
\hline $\mathrm{C} 2-\mathrm{H} 2$ & 1.1 & 3.9 & 1.1 & 2.6 & 1.3 \\
\hline $\mathrm{C} 3-\mathrm{H} 3$ & 13.5 & 11.6 & 13.4 & 13.4 & 13.6 \\
\hline $3-\mathrm{CH}_{3}$ & -8.8 & 0.7 & -8.5 & 0.8 & -7.1 \\
\hline $\mathrm{C} 4-\mathrm{H} 4$ & -4.1 & -1.7 & -4.1 & -2.4 & -4.2 \\
\hline $5-\mathrm{CH}_{3}$ & 10.2 & 4.5 & 10.5 & 5.0 & 12.8 \\
\hline C6-H6 & 12.0 & 12.5 & 11.9 & 12.5 & 11.6 \\
\hline$Q$ factor & & 0.69 & 0.04 & 0.58 & 0.20 \\
\hline Coupling & $D_{\operatorname{Exp}} / \mathrm{Hz}$ & $D_{\text {calc }}(R S R-e q) / \mathrm{Hz}$ & $D_{\text {Calc }}(R S R-a x) / \mathrm{Hz}$ & $D_{\text {Calc }}($ RSS-eq $) / \mathrm{Hz}$ & $D_{\text {calc }}(R S S-a x) / \mathrm{Hz}$ \\
\hline $1-\mathrm{CH}_{3}$ & -8.9 & -7.3 & 0.9 & -10.8 & 1.7 \\
\hline $\mathrm{C} 2-\mathrm{H} 2$ & 1.1 & 3.3 & 0.3 & 2.9 & 0.7 \\
\hline $\mathrm{C} 3-\mathrm{H} 3$ & 13.5 & 12.9 & 13.9 & 12.7 & 12.5 \\
\hline $3-\mathrm{CH}_{3}$ & -8.8 & -0.2 & -0.4 & -4.5 & -8.7 \\
\hline $\mathrm{C} 4-\mathrm{H} 4$ & -4.1 & -2.8 & -3.1 & -2.9 & -3.5 \\
\hline $5-\mathrm{CH}_{3}$ & 10.2 & 2.0 & 6.8 & 2.5 & 13.2 \\
\hline C6-H6 & 12.0 & 12.9 & 12.2 & 13.0 & 11.5 \\
\hline$Q$ factor & & 0.50 & 0.55 & 0.38 & 0.45 \\
\hline
\end{tabular}

MMFF9

\begin{tabular}{cccccc} 
Coupling & $D_{\text {Exp }} / \mathrm{Hz}$ & $D_{\text {Calc }}(S S R-e q) / \mathrm{Hz}$ & $D_{\text {Calc }}(S S R-a x / \mathrm{Hz}$ & $D_{\text {Calc }}(S S S-e q) / \mathrm{Hz}$ & $D_{\text {calc }}(S S S-a x) / \mathrm{Hz}$ \\
\hline $1-\mathrm{CH}_{3}$ & -8.9 & -0.4 & -8.7 & -1.6 & -5.9 \\
$\mathrm{C} 2-\mathrm{H} 2$ & 1.1 & 3.9 & 1.1 & 3.4 & 1.3 \\
$\mathrm{C} 3-\mathrm{H} 3$ & 13.5 & 11.9 & 13.4 & 12.5 & 13.5 \\
$3-\mathrm{CH}_{3}$ & -8.8 & 3.0 & -8.6 & 2.6 & -7.2 \\
$\mathrm{C} 4-\mathrm{H} 4$ & -4.1 & -1.3 & -4.1 & -2.2 & -4.1 \\
$5-\mathrm{CH}_{3}$ & 10.2 & 5.4 & 11.0 & 4.7 & 12.9 \\
$\mathrm{C} 6-\mathrm{H} 6$ & 12.0 & 12.6 & 11.9 & 12.8 & 11.6 \\
Q factor & & 0.65 & 0.05 & 0.61 & 0.18 \\
\hline
\end{tabular}




\begin{tabular}{cccccc}
\hline Coupling & $D_{\text {Exp }} / \mathrm{Hz}$ & $D_{\text {Calc }}(R S R-e q) / \mathrm{Hz}$ & $D_{\text {Calc }}(R S R-a x) / \mathrm{Hz}$ & $D_{\text {Calc }}(R S S-e q) / \mathrm{Hz}$ & $D_{\text {Calc }}(R S S-a x) / \mathrm{Hz}$ \\
\hline $1-\mathrm{CH}_{3}$ & -8.9 & -7.4 & 0.6 & -11.5 & 0.6 \\
$\mathrm{C} 2-\mathrm{H} 2$ & 1.1 & 3.4 & 0.4 & 2.0 & 0.7 \\
$\mathrm{C} 3-\mathrm{H} 3$ & 13.5 & 12.9 & 13.9 & 13.2 & 12.5 \\
$3-\mathrm{CH}_{3}$ & -8.8 & -1.2 & -0.7 & -6.3 & -8.5 \\
$\mathrm{C} 4-\mathrm{H} 4$ & -4.1 & -2.7 & -3.1 & -3.3 & -3.7 \\
$5-\mathrm{CH}_{3}$ & 10.2 & 1.4 & 7.6 & 5.4 & 13.8 \\
$\mathrm{C} 6-\mathrm{H} 6$ & 12.0 & 13.0 & 12.2 & 12.6 & 11.5 \\
Qfactor & & 0.49 & 0.52 & 0.25 & 0.42
\end{tabular}

Unoptimized

\begin{tabular}{|c|c|c|c|c|c|}
\hline Coupling & $D_{\text {Exp }} / \mathrm{Hz}$ & $D_{\text {Calc }}(S S R-e q) / \mathrm{Hz}$ & $D_{\text {Calc }}(S S R-a x / \mathrm{Hz}$ & $D_{\text {Calc }}(S S S-e q) / \mathrm{Hz}$ & $D_{\text {Calc }}(S S S-a x) / \mathrm{Hz}$ \\
\hline $1-\mathrm{CH}_{3}$ & -8.9 & 5.0 & -8.5 & 0.8 & -6.7 \\
\hline $\mathrm{C} 2-\mathrm{H} 2$ & 1.1 & 2.6 & 1.1 & 2.4 & 1.3 \\
\hline $\mathrm{C} 3-\mathrm{H} 3$ & 13.5 & 11.8 & 13.3 & 13.6 & 13.6 \\
\hline $3-\mathrm{CH}_{3}$ & -8.8 & -7.3 & -8.3 & -0.2 & -7.8 \\
\hline $\mathrm{C} 4-\mathrm{H} 4$ & -4.1 & -1.8 & -4.1 & -2.4 & -4.2 \\
\hline $5-\mathrm{CH}_{3}$ & 10.2 & 9.1 & 11.6 & 5.9 & 12.3 \\
\hline C6-H6 & 12.0 & 12.2 & 11.8 & 12.4 & 11.6 \\
\hline$Q$ factor & & 0.59 & 0.07 & 0.56 & 0.14 \\
\hline Coupling & $D_{\text {Exp }} / \mathrm{Hz}$ & $D_{\text {Calc }}(R S R-e q) / \mathrm{Hz}$ & $D_{\text {Calc }}(R S R-a x) / \mathrm{Hz}$ & $D_{\text {Calc }}(R S S-e q) / \mathrm{Hz}$ & $D_{\text {Calc }}(R S S-a x) / \mathrm{Hz}$ \\
\hline $1-\mathrm{CH}_{3}$ & -8.9 & -10.7 & 1.3 & -10.8 & 1.8 \\
\hline $\mathrm{C} 2-\mathrm{H} 2$ & 1.1 & 1.4 & 0.3 & 1.4 & 0.5 \\
\hline $\mathrm{C} 3-\mathrm{H} 3$ & 13.5 & 13.3 & 14.0 & 13.4 & 12.2 \\
\hline $3-\mathrm{CH}_{3}$ & -8.8 & -7.9 & -2.1 & -7.2 & -8.9 \\
\hline $\mathrm{C} 4-\mathrm{H} 4$ & -4.1 & -3.7 & -3.5 & -3.7 & -3.3 \\
\hline $5-\mathrm{CH}_{3}$ & 10.2 & 8.8 & 8.1 & 8.1 & 13.3 \\
\hline C6-H6 & 12.0 & 12.2 & 12.0 & 12.3 & 11.6 \\
\hline$Q$ factor & & 0.11 & 0.51 & 0.14 & 0.46 \\
\hline
\end{tabular}




\section{Exemplary MSpin Input and Output}

Input file:

rdc_data \{

$216-11.4$

31711.9

61812.7

92110.2

9204.5

81916.1

$1425-6.5$

$1426-6.5$

$1427-6.5$

15286.6

15296.6

15306.6

\}

permutations \{

2021

\}

Output file:

Solution 1

Conformer 1

Distribution size $=128$

Distribution type $=$ Gaussian

RDC general Std.Dev $=0.5 \mathrm{~Hz}$

Alignment tensor

$\left\langle\mathrm{A}^{\prime} \mathrm{x}\right\rangle=-1.369 \mathrm{e}-04$ Std.Dev $=6.718 \mathrm{e}-06$

$\left\langle\mathrm{A}^{\prime} \mathrm{y}\right\rangle=-3.696 \mathrm{e}-04$ Std.Dev $=9.399 \mathrm{e}-06$

$\left\langle\mathrm{A}^{\prime} \mathrm{z}\right\rangle=5.065 \mathrm{e}-04$ Std.Dev $=8.449 \mathrm{e}-06$

Quality factors statistic

$<\mathrm{Q}>=0.203$

$\operatorname{StdDev}(\mathrm{Q})=0.012$

Highest $\mathrm{Q}=0.231$

Lowest $\mathrm{Q}=0.169$

\section{Structural Noise Script}

filename = raw_input("Enter xyz file name ")

n_fixed_atoms = input ("Enter number of coordinates to vary (one block at the end of the file) ")

n_distances = input ("Enter number of radii you want to apply ")

distances $=[]$

for_in range (n_distances):

random_distance = input ("Enter radius of sphere in Angstrom (random displacement of atoms within this sphere) ") distances.append(random_distance)

molecules_varied = input ("Enter desired number of molecules for each distance ")

for $\mathrm{k}$ in range (n_distances):

atoms $=[]$

coordinates $=[]$

xyz_startmolecule = open $(\operatorname{str}($ os.path.realpath("_file__") $[:-8])+\operatorname{str}($ filename $)+" . x y z "$, "r")

n_atoms $=$ int $\left(x y z \_s t a r t m o l e c u l e . r e a d l i n e()\right)$

title $=$ xyz_startmolecule.readline ()

for line in xyz_startmolecule:

atom,x_coordinate,y_coordinate,z_coordinate $=$ line.split ()

atoms.append(atom)

coordinates.append([float( $x_{-}$coordinate), float(y_coordinate), float (z_coordinate) $]$)

xyz_varied = open(str(os.path.realpath("_file_")[:-8])+str(filename)+"_var_"+str(distances[k])+".xyz", "a")

for_in range (int(molecules_varied)):

xyz_varied.write(str(n_atoms)+"In|n")

for $\mathrm{i}$ in range ((n_atoms)): 
xyz_varied.write(str(atoms[i])+" ")

if $\mathrm{i}<$ (n_atoms $-\mathrm{n} \_$fixed_atoms):

for $\mathrm{j}$ in range (3):

old_coods $="\{0.5 f\} "$.format $($ coordinates $[\mathrm{i}][\mathrm{j}])$

xyz_varied.write(str(old_coods)+" ")

xyz_varied.write("In")

else:

$\mathrm{u}=$ random.uniform $(0,1)$

$\mathrm{r}=\operatorname{distances}[\mathrm{k}]^{*}$ sp.special.cbrt $(\mathrm{u})$

costheta $=$ random.uniform $(-1,1)$

theta $=\mathrm{np} \cdot \arccos (\cos$ theta $)$

$\mathrm{phi}=$ random.uniform $\left(0,2^{\star} \mathrm{np} \cdot \mathrm{pi}\right)$

new_x $=$ "\{0:.5f $\}$ ".format (float $($ coordinates $\left.[\mathrm{i}][0])+\mathrm{r}^{*} \mathrm{np} \cdot \sin (\text { theta })^{*} \mathrm{np} \cdot \cos (\mathrm{phi})\right)$

new_y $="\{0: 5 f\} "$.format (float (coordinates $[\mathrm{i}][1])+\mathrm{r}^{*} \mathrm{np} \cdot \sin (\text { theta })^{*} \mathrm{np} \cdot \sin ($ phi $\left.)\right)$

new $\_z="\{0: 5 f\}$ ".format(float (coordinates $\left.[i][2]\right)+r^{*} n p \cdot \cos ($ theta $\left.)\right)$

xyz_varied.write("|n")

xyz_varied.write(str(new_x)+" " $+\operatorname{str}\left(n e w \_y\right)+" \quad$ "+str(new_z)+"|n")

xyz_varied.close()

xyz_startmolecule.close() 


\section{Extended NMR Spectroscopy of Synthetic Indanes}

\section{$J$-coupling Analysis of $\beta$-aa-H and $\beta$-aa-Me}

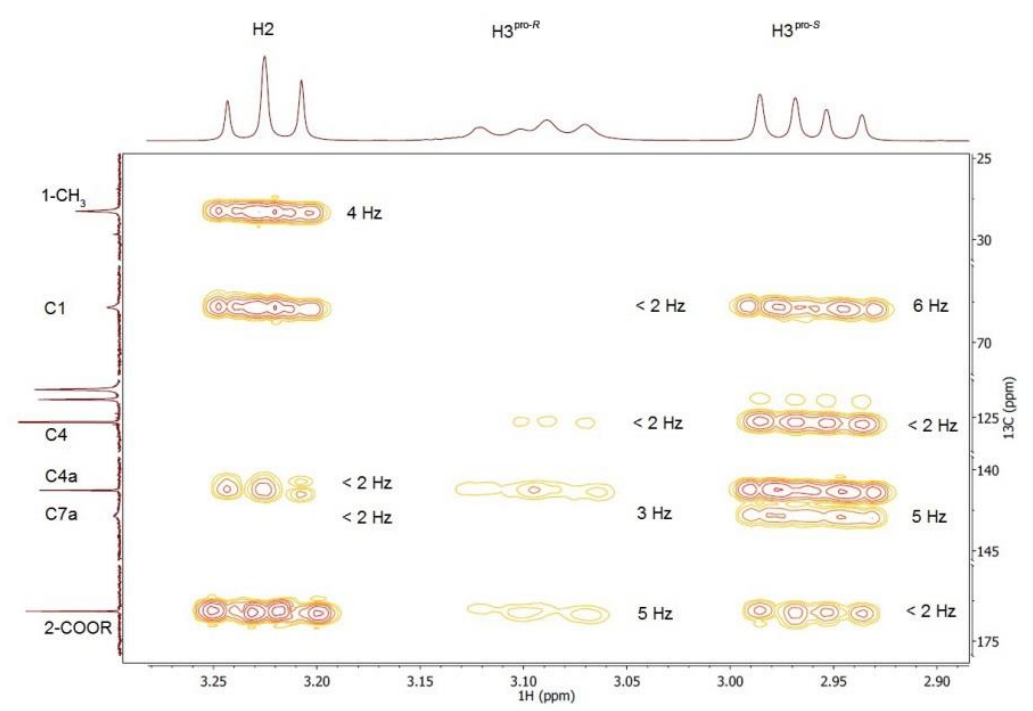

Figure B-5: Excerpt of HMBC spectrum of $\beta$-aa-H. For listed $J$-couplings see Table B-2.

Table B-2: Comparison of dihedral angles (in ${ }^{\circ}$ ) in the two conformations (ester group on C2 equatorial or axial) of the $S S$ and $R S$ isomers of $\beta$-aa-H with experimental 3-bond coupling constants as estimated from highresolution HMBC experiments. The structures were generated with MM2 geometry optimization. ${ }^{[151]}$ Angles that are at variance with the experiment are labeled red. Angles that are at variance with the experiment if the assignment of $\mathrm{H} 3^{\text {pro-s }}(2.96 \mathrm{ppm})$ and $\mathrm{H} 3^{\text {pro- } R}(3.09 \mathrm{ppm})$ is swapped are labeled blue.

\begin{tabular}{|c|c|c|c|c|c|}
\hline \multirow{2}{*}{ Atoms } & \multicolumn{2}{|c|}{ Dihedral Angle } & \multicolumn{2}{|c|}{ Dihedral Angle } & \multirow{2}{*}{${ }^{3} J_{\mathrm{HH}}$ or ${ }^{3} J_{\mathrm{CH}} / \mathrm{Hz}$} \\
\hline & SS-eq & $S S-a x$ & $R S-e q$ & $R S-a x$ & \\
\hline $\mathrm{H} 2-\mathrm{C} 2-\mathrm{C} 3-\mathrm{H} 3^{\text {pro-S }}$ & 31.8 & -33.1 & 34.2 & -34.0 & 8.5 \\
\hline $\mathrm{H} 2-\mathrm{C} 2-\mathrm{C} 3-\mathrm{H} 3^{\text {pro- } R}$ & 156.1 & 90.7 & 158.7 & 89.6 & 9.3 \\
\hline H2-C2-C3-C4a & -89.0 & -148.7 & -86.3 & -149.3 & $<2$ \\
\hline H2-C2-C1-C7a & 88.5 & 149.8 & 84.8 & 149.1 & $<2$ \\
\hline $\mathrm{H} 2-\mathrm{C} 2-\mathrm{C} 1-\left(1-\mathrm{CH}_{3}\right)$ & -31.9 & 32.6 & -157.3 & -87.8 & $\sim 5$ \\
\hline $\mathrm{H} 3^{\text {pro-s}}-\mathrm{C} 3-\mathrm{C} 2-\mathrm{CO}_{2} \mathrm{Me}$ & -84.2 & -151.2 & -83.6 & -152.0 & $<2$ \\
\hline $\mathrm{H} 3^{\text {pro-R}}-\mathrm{C} 3-\mathrm{C} 2-\mathrm{CO}_{2} \mathrm{Me}$ & 40.2 & -27.4 & 40.9 & -29.4 & $\sim 5$ \\
\hline $\mathrm{H} 3^{\text {pro-s }}-\mathrm{C} 3-\mathrm{C} 2-\mathrm{C} 1$ & 146.7 & 86.9 & 148.8 & 86.2 & $\sim 6$ \\
\hline $\mathrm{H} 3^{\mathrm{pro}-\mathrm{R}}-\mathrm{C} 3-\mathrm{C} 2-\mathrm{C} 1$ & -89.0 & -149.4 & -86.7 & -150.3 & $<2$ \\
\hline $\mathrm{H} 3^{\text {pro-s }}-\mathrm{C} 3-\mathrm{C} 4 \mathrm{a}-\mathrm{C} 4$ & 42.7 & 81.4 & 41.5 & 81.8 & $<2$ \\
\hline $\mathrm{H} 3^{\text {pro-R}}-\mathrm{C} 3-\mathrm{C} 4 \mathrm{a}-\mathrm{C} 4$ & -78.9 & -40.2 & -80.2 & -39.9 & $<2$ \\
\hline $\mathrm{H} 3^{\text {pro-s }}-\mathrm{C} 3-\mathrm{C} 4 \mathrm{a}-\mathrm{C} 7 \mathrm{a}$ & -137.2 & -99.3 & -138.2 & -99.2 & $\sim 5$ \\
\hline $\mathrm{H} 3^{\text {pro-R }}-\mathrm{C} 3-\mathrm{C} 4 \mathrm{a}-\mathrm{C} 7 \mathrm{a}$ & 101.2 & 139.1 & 100.2 & 139.2 & $\sim 3$ \\
\hline
\end{tabular}




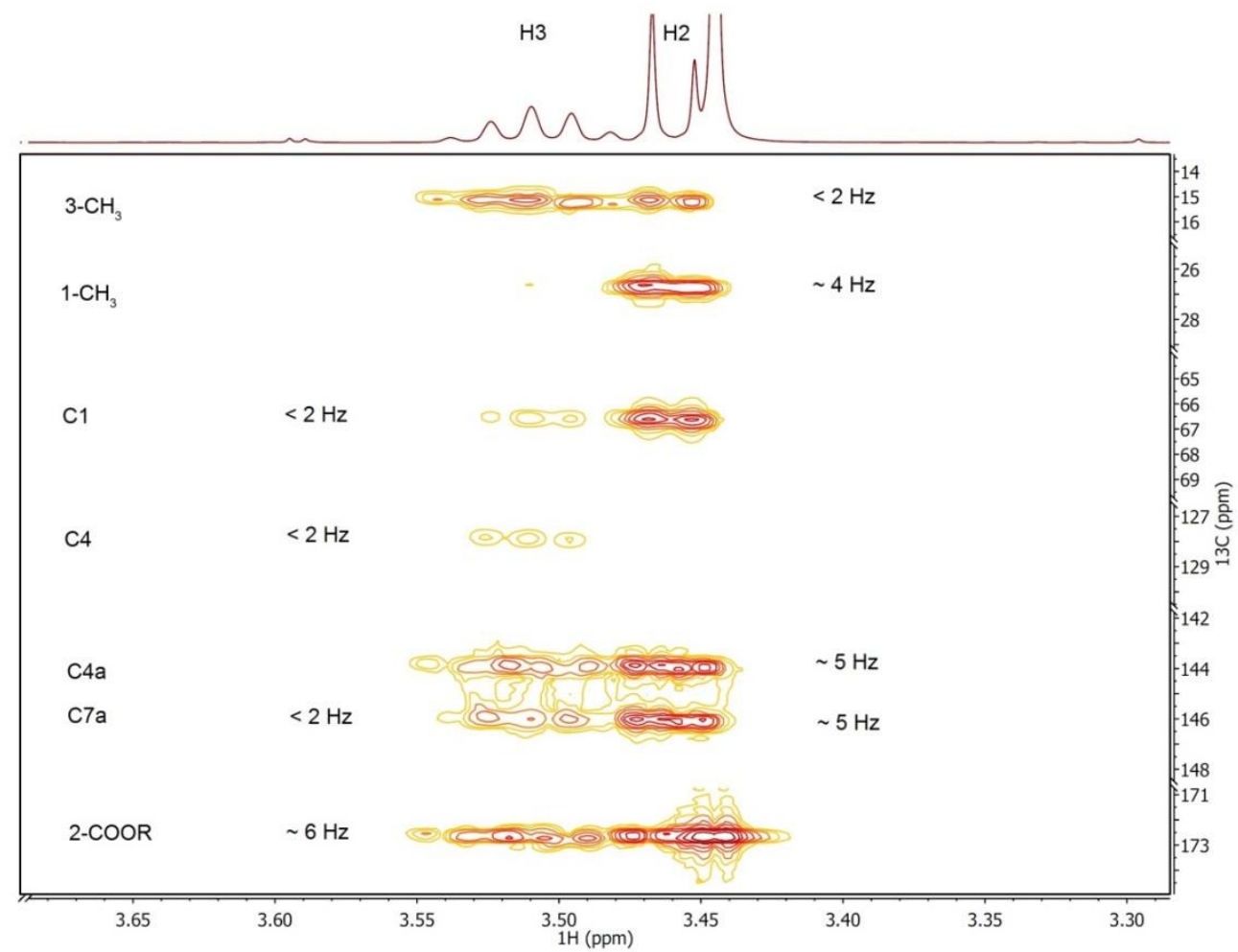

Figure B-6: Excerpt of HMBC spectrum of $\beta$-aa-Me. For listed $J$-couplings see Table B-3.

Table B-3: Comparison of dihedral angles (in ${ }^{\circ}$ ) in the two conformations (ester group on C2 equatorial or axial) of SSR, SSS, RSR and RSS isomers of $\beta$-aa-Me with experimental 3-bond coupling constants as estimated from high-resolution HMBC experiments. The structures were generated with MM2 geometry optimization. ${ }^{[151]}$ Angles that are at variance with the experiment are labeled red.

\begin{tabular}{|c|c|c|c|c|c|c|c|c|c|}
\hline \multirow{2}{*}{ Atoms } & \multicolumn{2}{|c|}{ Dihedral Angle } & \multicolumn{2}{|c|}{ Dihedral Angle } & \multicolumn{2}{|c|}{ Dihedral Angle } & \multicolumn{2}{|c|}{ Dihedral Angle } & \multirow{2}{*}{${ }^{3} J_{\mathrm{HX}} / \mathrm{Hz}$} \\
\hline & SSR-eq & SSR-ax & SSS-eq & SSS-ax & RSR-eq & RSR-ax & RSS-eq & RSS-ax & \\
\hline $\mathrm{H} 2-\mathrm{C} 2-\mathrm{C} 3-\mathrm{H} 3$ & 29.3 & -35.6 & 156.7 & 103.6 & 31.6 & -38.5 & 162.6 & 96.6 & 7.4 \\
\hline $\mathrm{H} 2-\mathrm{C} 2-\mathrm{C} 3-\left(3-\mathrm{CH}_{3}\right)$ & 153.4 & 87.2 & 32.7 & -19.4 & 156.9 & 83.9 & 37.9 & -26.4 & $<2$ \\
\hline $\mathrm{H} 2-\mathrm{C} 2-\mathrm{C} 3-\mathrm{C} 4 \mathrm{a}$ & -87.2 & -148.1 & -90.6 & -139.8 & -84.0 & -150.7 & -84.5 & -146.0 & $\sim 5$ \\
\hline $\mathrm{H} 2-\mathrm{C} 2-\mathrm{C} 1-\mathrm{C} 7 \mathrm{a}$ & 86.9 & 148.6 & 90.4 & 140.9 & 83.4 & 150.6 & 83.6 & 145.9 & $\sim 5$ \\
\hline $\mathrm{H} 2-\mathrm{C} 2-\mathrm{Cl}-\left(1-\mathrm{CH}_{3}\right)$ & -33.6 & 31.1 & -30.0 & 22.6 & -158.6 & -85.9 & -159.0 & -91.3 & $\sim 4$ \\
\hline $\mathrm{H} 3-\mathrm{C} 3-\mathrm{C} 2-\mathrm{CO}_{2} \mathrm{Me}$ & -83.0 & -155.0 & 39.8 & -14.0 & -80.9 & -158.4 & 162.6 & -21.5 & $\sim 6$ \\
\hline $\mathrm{H} 3-\mathrm{C} 3-\mathrm{C} 2-\mathrm{C} 1$ & 141.3 & 83.6 & -88.3 & -136.3 & 142.6 & 80.4 & -83.8 & -142.6 & $<2$ \\
\hline $\mathrm{H} 3-\mathrm{C} 3-\mathrm{C} 4 \mathrm{a}-\mathrm{C} 4$ & 47.8 & 84.2 & -79.4 & -49.9 & 47.3 & 86.5 & -82.2 & -46.2 & $<2$ \\
\hline H3-C3-C4a-C7a & -132.1 & -95.7 & 100.0 & 130.0 & -137.7 & -93.9 & 97.8 & 133.5 & $<2$ \\
\hline
\end{tabular}


NOE Analysis of $\beta$-aa-H and $\beta$-aa-Me

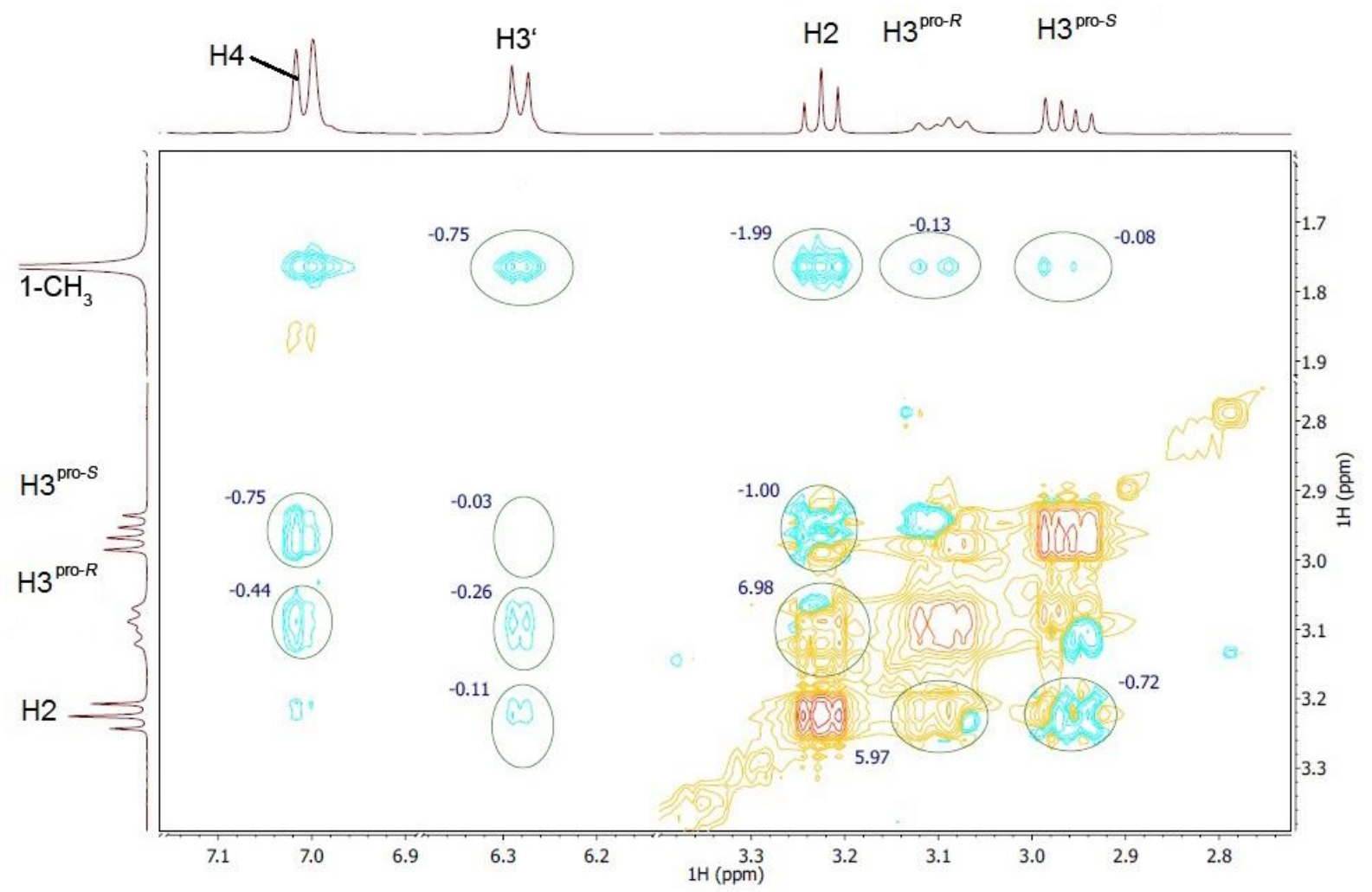

Figure B-7: Excerpt of NOESY spectrum of $\beta$-aa-H. For listed NOE contacts see Table B-4.

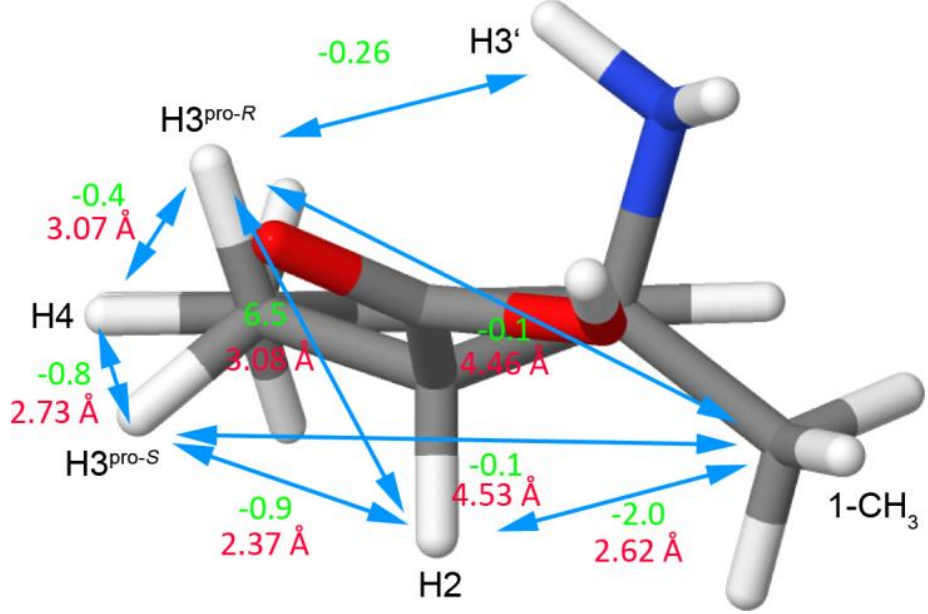

Figure B-8: Correct structure SS-eq (MM2 optimized $\left.{ }^{[151]}\right)$. Green: NOE Integral. Red: Distances. 
Table B-4: Comparison of inter-proton distances in the two conformations (ester group on C2 equatorial or axial) of the $S S$ and $R S$ isomers of $\beta-$ aa-H with experimental NOE contacts as estimated from NOESY integrals. The structures were generated with MM2 geometry optimization. ${ }^{[151]}$ For methyl groups, the position of the carbon atom was used.

\begin{tabular}{|c|c|c|c|c|c|}
\hline & \multicolumn{2}{|c|}{ Distances $/ \AA ̊$} & \multicolumn{2}{|c|}{ Distances $/ \AA$} & \multirow[t]{2}{*}{ NOE Integra } \\
\hline & SS-eq & SS- $a x$ & $R S-e q$ & $R S-a x$ & \\
\hline$\left(1-\mathrm{CH}_{3}\right)-\mathrm{H} 2$ & 2.62 & 2.63 & 3.49 & 3.11 & -2.0 \\
\hline$\left(1-\mathrm{CH}_{3}\right)-\mathrm{H} 3^{\text {pro-S }}$ & 4.53 & 3.20 & 4.36 & 4.43 & -0.1 \\
\hline$\left(1-\mathrm{CH}_{3}\right)-\mathrm{H} 3^{\text {pro- } R}$ & 4.46 & 4.32 & 3.27 & 4.62 & -0.1 \\
\hline $\mathrm{H} 2-\mathrm{H} 3^{\text {pro-s }}$ & 2.37 & 2.44 & 2.37 & 2.42 & -0.9 \\
\hline $\mathrm{H} 2-\mathrm{H} 3^{\mathrm{pro}-\mathrm{R}}$ & 3.08 & 2.81 & 3.08 & 2.79 & 6.5 \\
\hline $\mathrm{H} 3^{\text {pro-s}}-\mathrm{H} 4$ & 2.73 & 3.13 & 2.74 & 3.13 & -0.8 \\
\hline $\mathrm{H} 3^{\text {pro-R }}-\mathrm{H} 4$ & 3.07 & 2.73 & 3.10 & 2.73 & -0.4 \\
\hline
\end{tabular}

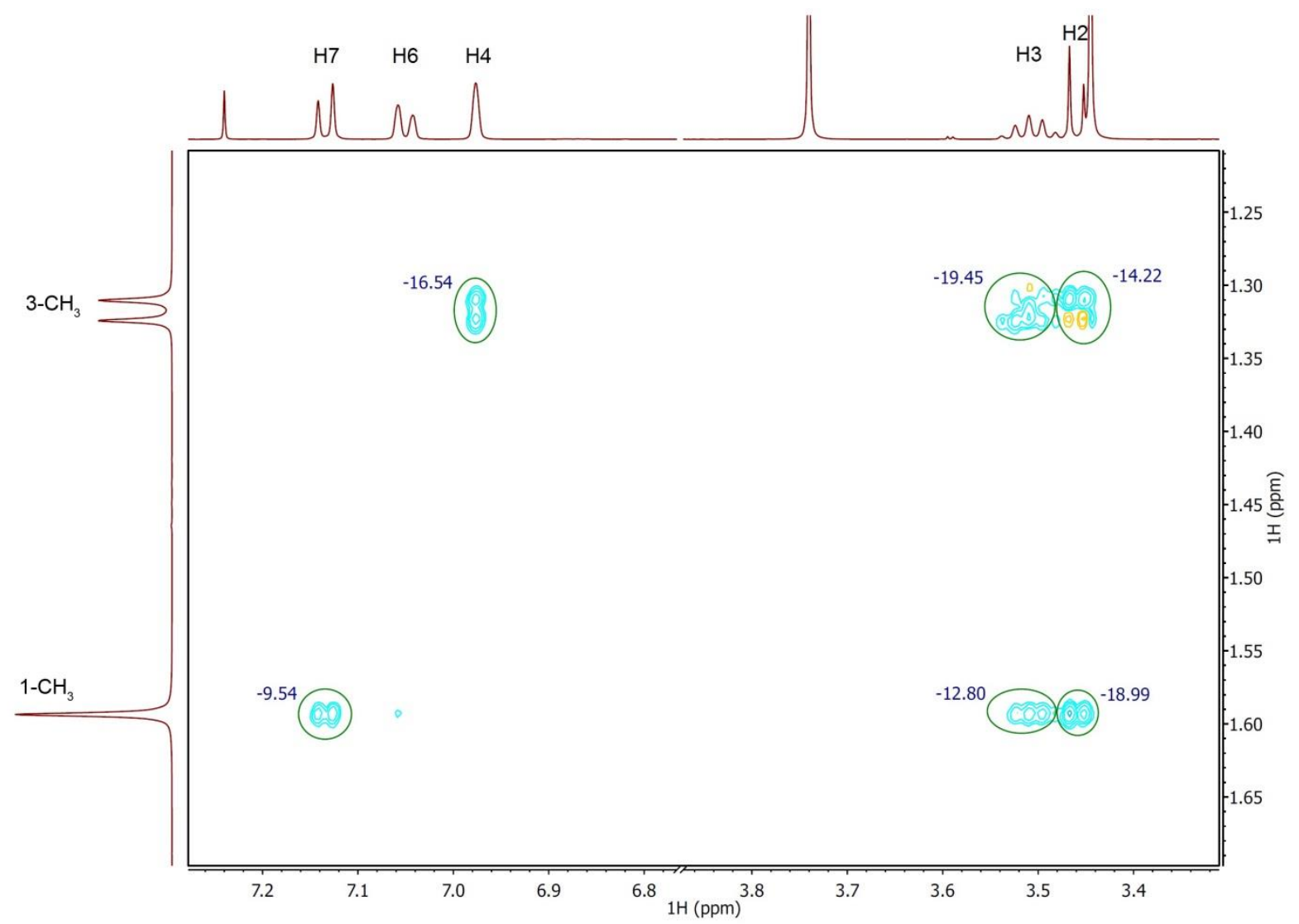

Figure B-9: Excerpt of NOESY spectrum of $\beta$-aa-Me. For listed NOE contacts see Table B-5. 


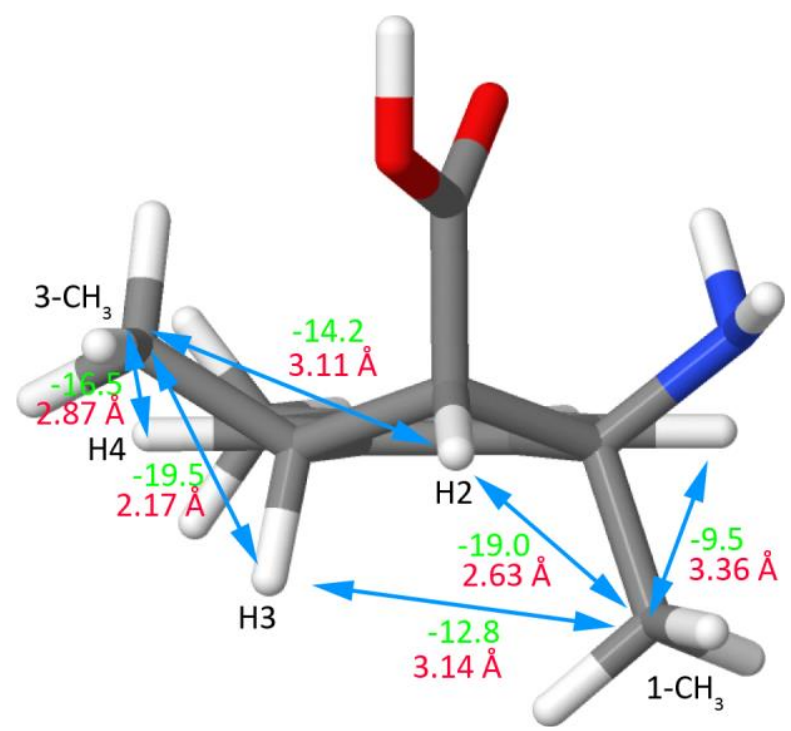

Figure B-10: Correct structure SSR-ax (MM2 optimized $\left.{ }^{[151]}\right)$. Green: NOE Integral. Red: Distances.

Table B-5: Comparison of inter-proton distances in the two conformations (ester group on C2 equatorial or axial) of the SSR, SSS, RSR and RSS isomers of $\beta$-aa-Me with experimental NOE contacts as estimated from NOESY integrals. The structures were generated with MM2 geometry optimization. For methyl groups, the position of the carbon atom was used.

\begin{tabular}{cccccccccc} 
& \multicolumn{2}{c}{ Distances $/ \AA$} & \multicolumn{2}{c}{ Distances $/ \AA$} & \multicolumn{2}{c}{ Distances $/ \AA$} & \multicolumn{2}{c}{ Distances $/ \AA$} & NOE \\
& SSR-eq & SSR-ax & SSS-eq & SSS-ax & RSR-eq & RSR-ax & RSS-eq & RSS-ax & Integral \\
$\left(1-\mathrm{CH}_{3}\right)-\mathrm{H} 2$ & 2.56 & 2.63 & 2.60 & 2.59 & 3.49 & 3.08 & 3.48 & 3.13 & -19.0 \\
$\left(1-\mathrm{CH}_{3}\right)-\mathrm{H} 3$ & 4.47 & 3.14 & 4.44 & 4.40 & 4.43 & 4.35 & 3.19 & 4.53 & -12.8 \\
$\left(1-\mathrm{CH}_{3}\right)-\mathrm{H} 7$ & 2.92 & 3.36 & 2.90 & 3.25 & 3.27 & 2.87 & 3.34 & 2.90 & -9.5 \\
$\left(3-\mathrm{CH}_{3}\right)-\mathrm{H} 2$ & 3.47 & 3.11 & 2.60 & 2.63 & 3.49 & 3.07 & 2.62 & 2.64 & -14.2 \\
$\left(3-\mathrm{CH}_{3}\right)-\mathrm{H} 3$ & 2.17 & 2.17 & 2.19 & 2.17 & 2.17 & 2.17 & 2.19 & 2.17 & -19.5 \\
$\left(3-\mathrm{CH}_{3}\right)-\mathrm{H} 4$ & 3.24 & 2.87 & 2.89 & 3.22 & 3.22 & 2.85 & 2.89 & 3.27 & -16.5
\end{tabular}

\section{Quantitative Analysis of Residual Quadrupolar Couplings Data}

\section{Exemplary ORCA input files EFG tensor calculation}

EFG gradient:

\# ORCA JOB on ORCA optimized structure

$\% \operatorname{maxcore} X$

\%pal nprocs $Y$

\#choose $\mathrm{X}$ and $\mathrm{Y}$ according to hardware

! RKS NORI B3LYP 6-311++G(d,p) Decontract Grid5 NOFINALGRID VERYTIGHTSCF SlowConv Cosmo(solvens)

${ }^{*}$ xyzfile A B NAME.xyz

\#coordinate file forcefield optimized structure; choose A and B according to charge and multiplicity

\%eprnmr nuclei = all Q $\{$ fgrad $\}$

\#choose nuclei Q to calculate EFG for

end 


\section{Structure Data of [CpLi(OMe $)],\left[\mathrm{CpLi}\left(\mathrm{OMe}_{2}\right)_{2}\right],\left[\mathrm{CpLi}\left(\mathrm{OMe}_{2}\right)_{3}\right]$ and $\left[\mathrm{Cp}_{2} \mathrm{Li}^{-}\right.$}

\begin{tabular}{|c|c|c|c|}
\hline$\left[\mathrm{CpLi}\left(\mathrm{OMe}_{2}\right)\right]$ & {$\left[\mathrm{CpLi}\left(\mathrm{OMe}_{2}\right)_{2}\right]$} & {$\left[\mathrm{CpLi}\left(\mathrm{OMe}_{2}\right)_{3}\right]$} & {$\left[\mathrm{Cp}_{2} \mathbf{L i}\right]^{-}$} \\
\hline C - 1.75665 .69084 .7673 & O 7.72736 .82893 .0828 & Li 12.54451 .88709 .1681 & $\mathrm{Li}-0.0001-0.00020 .0000$ \\
\hline C - 2.06696 .97585 .2737 & O 7.95405 .18350 .5477 & O 11.15780 .33799 .5584 & C - 0.85560 .59712 .0554 \\
\hline C - 2.81146 .80786 .4655 & C 5.05244 .39242 .9735 & O 12.51362 .388211 .1430 & C - 0.60461 .77021 .3074 \\
\hline C - 2.96255 .41996 .6951 & C 4.75085 .71283 .3770 & O 14.41720 .92009 .5003 & C - 1.44311 .75040 .1687 \\
\hline C - 2.30674 .72965 .6473 & C 4.42856 .45912 .2243 & C 12.94043 .94908 .1842 & C - 2.21340 .56420 .2144 \\
\hline Li -3.9368 5.9480 4.8130 & C 4.51245 .59671 .1045 & C 13.46172 .97007 .3101 & C - $1.8510-0.14621 .3810$ \\
\hline O -5.7251 6.49604 .3190 & C 4.89094 .31661 .5693 & C 12.38362 .19736 .8231 & H - 0.35030 .30242 .9650 \\
\hline C -6.3574 6.34043.0509 & C 8.26676 .30524 .2965 & C 11.19282 .71017 .3845 & Н 0.12252 .53361 .5483 \\
\hline C -6.6395 6.46695 .4170 & C 8.62264 .02951 .0516 & C 11.53503 .78638 .2300 & $\mathrm{H}-1.47002 .4959-0.6141$ \\
\hline H -3.1994 7.6005 7.0888 & C $7.47064 .9713-0.7772$ & C $10.9786-0.69988 .6083$ & H - $2.93040 .2406-0.5273$ \\
\hline $\mathrm{H}-2.22553 .65675 .5416$ & C 8.66497 .66412 .4035 & C 9.93200 .719410 .1687 & H - $2.2473-1.10261 .6929$ \\
\hline H - 1.18625 .47723 .8747 & Li 6.53495 .75221 .9701 & C 12.76721 .390612 .1251 & C $0.8574-0.5939-2.0553$ \\
\hline H - 1.77807 .91754 .8290 & H 5.34483 .58073 .6248 & C 12.28913 .665411 .7229 & C $0.6041-1.7686-1.3108$ \\
\hline H -3.4819 4.9678 7.5280 & H 4.79896 .09704 .3864 & C 15.45671 .724210 .0421 & C $1.4411-1.7527-0.1709$ \\
\hline H -5.5815 6.4211 2.2922 & H 4.17157 .50912 .2001 & C $14.9203-0.00618 .5476$ & C $2.2127-0.5671-0.2125$ \\
\hline H -7.0990 7.1287 2.9014 & H 4.31865 .87520 .0783 & H 13.51674 .67408 .7421 & C $1.85270 .1466-1.3777$ \\
\hline H -6.8452 5.36392 .9792 & H 5.04483 .43780 .9606 & H 14.50662 .81577 .0799 & Н $0.3535-0.2962-2.9648$ \\
\hline H -6.0585 6.63056 .3227 & H 8.59357 .11894 .9503 & H 12.45951 .35436 .1507 & H - $0.1236-2.5305-1.5545$ \\
\hline H -7.1420 5.4973 5.4719 & H 7.47995 .73274 .7813 & H 10.19712 .32187 .2220 & H $1.4660-2.50020 .6101$ \\
\hline \multirow[t]{19}{*}{ H -7.3835 7.2601 5.3109 } & H 7.93063 .18511 .1270 & H 10.84414 .36828 .8244 & Н $2.9288-0.24610 .5312$ \\
\hline & H 9.45863 .75540 .4017 & H $11.9538-0.94268 .1928$ & H $2.25051 .1033-1.6866$ \\
\hline & H $6.70874 .1875-0.7913$ & H $10.3181-0.37577 .7982$ & \\
\hline & H 7.0293 5.9066 -1.1139 & Н $10.5547-1.58989 .0863$ & \\
\hline & Н 9.00408 .46743 .0637 & Н 10.15291 .526710 .8612 & \\
\hline & H 9.52857 .08382 .0654 & H $9.4952-0.125310 .7126$ & \\
\hline & H 9.12275 .65294 .0930 & H 9.22111 .07289 .4157 & \\
\hline & H 9.00354 .27682 .0408 & H 12.92070 .452511 .6003 & \\
\hline & H $8.29514 .6879-1.4379$ & H 11.91821 .299612 .8103 & \\
\hline & H 8.15858 .09151 .5410 & H 13.66201 .637212 .7063 & \\
\hline & & H 12.06754 .354410 .9145 & \\
\hline & & H 13.17844 .001912 .2667 & \\
\hline & & H 11.44463 .628012 .4193 & \\
\hline & & H 14.99822 .433310 .7248 & \\
\hline & & H 15.97532 .27219 .2487 & \\
\hline & & H 16.17981 .102310 .5812 & \\
\hline & & H $14.0784-0.56188 .1422$ & \\
\hline & & H $15.6215-0.70109 .0221$ & \\
\hline & & Н 15.42880 .51657 .7313 & \\
\hline
\end{tabular}




\section{References}

[1] T. Niklas, D. Stalke, M. John, Chem. Commun. 2015, 51, 1275-1277.

[2] T. Niklas, C. Steinmetzger, W. Liu, D. Zell, D. Stalke, L. Ackermann, M. John, Eur. J. Org. Chem. 2015, 2015, 6801-6805.

[3] W. Schlenk, J. Holtz, Ber. Dtsch. Chem. Ges. 1917, 50, 262-274.

[4] C. W. Kamienski, Ind. Eng. Chem. 1965, 57, 38-55.

[5] G. Wu, M. Huang, Chem. Rev. 2006, 106, 2596-2616.

[6] J. N. Reed, V. Snieckus, Tetrahedron Lett. 1983, 24, 3795-3798.

[7] J. N. Reed, Ph. D. Thesis, University of Waterloo (Canada), 1985.

[8] M. P. Sibi, S. Chattopadhyay, J. W. Dankwardt, V. Snieckus, J. Am. Chem. Soc. 1985, 107, 6312-6315.

[9] R. J. Mills, N. J. Taylor, V. Snieckus, J. Org. Chem. 1989, 54, 4372-4385.

[10] P. Beak, R. A. Brown, J. Org. Chem. 1982, 47, 34-46.

[11] P. E. Eaton, R. M. Martin, J. Org. Chem. 1988, 53, 2728-2732.

[12] H. Gilman, R. L. Bebb, J. Am. Chem. Soc. 1939, 61, 109-112.

[13] G. Wittig, G. Fuhrmann, Ber. Dtsch. Chem. Ges. 1940, 73, 1197-1218.

[14] T. Kottke, D. Stalke, J. Appl. Crystallogr. 1993, 26, 615-619.

[15] a) V. H. Gessner, C. Däschlein, C. Strohmann, Chem. Eur. J. 2009, 15, 3320-3334 b) H. J. Reich, Chem. Rev. 2013, 113, 7130-7178 c) E. Carl, D. Stalke, in Lithium Compounds in Organic Synthesis, Wiley-VCH, Weinheim, 2014, pp. 1-32.

[16] T. Kottke, D. Stalke, Angew. Chem. 1993, 105, 619-621, Angew. Chem. Int. Ed. 1993, 32, 580-582.

[17] a) S. Merkel, D. Stern, J. Henn, D. Stalke, Angew. Chem. 2009, 121, 6468-6471, Angew. Chem. Int. Ed. 2009, 48, 6350-6353 b) A.-C. Pöppler, M. Granitzka, R. Herbst-Irmer, Y.-S. Chen, B. B. Iversen, M. John, R. A. Mata, D. Stalke, Angew. Chem. 2014, 126, 13498-13503, Angew. Chem. Int. Ed. 2014, 53, 13282-13287 c) D. Pinchuk, J. Mathew, A. Kaushansky, D. Bravo-Zhivotovskii, Y. Apeloig, Angew. Chem. 2016, 128, 1041410418, Angew. Chem. Int. Ed. 2016, 55, 10258-10262 d) R. Michel, R. Herbst-Irmer, D. Stalke, Organometallics 2010, 29, 6169-6171 e) R. Michel, R. Herbst-Irmer, D. Stalke, Organometallics 2011, 30, 4379-4386.

[18] M. A. Nichols, P. G. Williard, J. Am. Chem. Soc. 1993, 115, 1568-1572.

[19] C. Strohmann, T. Seibel, K. Strohfeldt, Angew. Chem. 2003, 115, 4669-4671, Angew. Chem. Int. Ed. 2003, 42, 4531-4533.

[20] a) J. Doulcet, G. R. Stephenson, Chem. Eur. J. 2015, 21, 18677-18689 b) J. D. Firth, P. O’Brien, L. Ferris, J. Am. Chem. Soc. 2016, 138, 651-659 c) P. J. Rayner, J. C. Smith, C. Denneval, P. O'Brien, P. A. Clarke, R. A. J. Horan, Chem. Commun. 2016, 52, 1354-1357. 
[21] a) J. S. DePue, D. B. Collum, J. Am. Chem. Soc. 1988, 110, 5524-5533 b) J. Liang, A. C. Hoepker, A. M. Bruneau, Y. Ma, L. Gupta, D. B. Collum, J. Org. Chem. 2014, 79, 11885-11902 c) E. H. Tallmadge, J. Jermaks, D. B. Collum, J. Am. Chem. Soc. 2016, 138, 345-355.

[22] a) D. B. Collum, Acc. Chem. Res. 1992, 25, 448-454 b) P. Zhao, D. B. Collum, J. Am. Chem. Soc. 2003, 125, 4008-4009.

[23] E. Weiss, E. A. C. Lucken, J. Organomet. Chem. 1964, 2, 197-205.

[24] M. Sebban, L. Guilhaudis, H. Oulyadi, in Lithium Compounds in Organic Synthesis, Wiley-VCH Weinheim, 2014, pp. 85-122.

[25] a) N. Sharma, J. K. Ajay, K. Venkatasubbaiah, U. Lourderaj, Phys. Chem. Chem. Phys. 2015, 17, 22204-22209 b) M. Casimiro, J. Garcia-Lopez, M. J. Iglesias, F. Lopez-Ortiz, Dalton Trans. 2014, 43, 14291-14301 c) V. Capriati, S. Florio, R. Luisi, B. Musio, I. Alkorta, F. Blanco, J. Elguero, Struct. Chem. 2008, 19, 785-792 d) P. O'Brien, K. B. Wiberg, W. F. Bailey, J.-P. R. Hermet, M. J. McGrath, J. Am. Chem. Soc. 2004, 126, 1548015489.

[26] W. Bauer, F. Hampel, J. Chem. Soc., Chem. Commun. 1992, 903-905.

[27] R. M. Gschwind, P. R. Rajamohanan, M. John, G. Boche, Organometallics 2000, 19, 2868-2873.

[28] a) G. Hamdoun, B. Gouilleux, M. Sebban, G. Barozzino-Consiglio, A. Harrison-Marchand, P. Giraudeau, J. Maddaluno, H. Oulyadi, Chem. Commun. 2017, 53, 220-223 b) G. Hamdoun, M. Sebban, V. Tognetti, A. Harrison-Marchand, L. Joubert, J. Maddaluno, H. Oulyadi, Organometallics 2015, 34, 1932-1941.

[29] a) J. F. McGarrity, J. Prodolliet, T. Smyth, Org. Magn. Resonance 1981, 17, 59-65 b) S. E. Denmark, B. J. Williams, B. M. Eklov, S. M. Pham, G. L. Beutner, J. Org. Chem. 2010, 75, 5558-5572 c) M. D. Christianson, E. H. P. Tan, C. R. Landis, J. Am. Chem. Soc. 2010, 132, 11461-11463.

[30] K. F. Morris, C. S. Johnson, J. Am. Chem. Soc. 1992, 114, 3139-3141.

[31] A. C. Jones, Rapid-injection NMR and Organolithium Reactivity, University of Wisconsin, Wisconsin, 2007.

[32] A. Einstein, Ann. Phys. 1905, 322, 549-560.

[33] I. Keresztes, P. G. Williard, J. Am. Chem. Soc. 2000, 122, 10228-10229.

[34] S. Bachmann, R. Neufeld, M. Dzemski, D. Stalke, Chem. Eur. J. 2016, 22, 8462-8465.

[35] D. Li, G. Kagan, R. Hopson, P. G. Williard, J. Am. Chem. Soc. 2009, 131, 5627-5634.

[36] R. Neufeld, D. Stalke, Chem. Sci. 2015, 6, 3354-3364.

[37] A.-C. Pöppler, S. Frischkorn, D. Stalke, M. John, ChemPhysChem 2013, 14, 3103-3107.

[38] A.-C. Pöppler, H. Keil, D. Stalke, M. John, Angew. Chem. 2012, 124, 7963-7967, Angew. Chem. Int. Ed. 2012, $51,7843-7846$.

[39] a) K. K. Kwong, J. W. Belliveau, D. A. Chesler, I. E. Goldberg, R. M. Weisskoff, B. P. Poncelet, D. N. Kennedy, B. E. Hoppel, M. S. Cohen, R. Turner, Proc. Natl. Acad. Sci. U. S. A. 1992, 89, 5675-5679 b) R. R. Edelman, S. Warach, N. Engl. J. Med. 1993, 328, 708-716 c) N. Weiskopf, R. Sitaram, O. Josephs, R. Veit, F. Scharnowski, R. Goebel, N. Birbaumer, R. Deichmann, K. Mathiak, Magn. Res. Imaging 2007, 25, 989-1003. 
[40] S. Grant, MRI: A Guided Tour can be found under https://nationalmaglab.org/education/magnetacademy/learn-the-basics/stories/mri-a-guided-tour, 23.02.2017.

[41] G. E. Wagner, P. Sakhaii, W. Bermel, K. Zangger, Chem. Commun. 2013, 49, 3155-3157.

[42] M. Vega-Vazquez, J. C. Cobas, M. Martin-Pastor, Magn. Reson. Chem. 2010, 48, 749-752.

[43] B. Sathyamoorthy, D. M. Parish, G. T. Montelione, R. Xiao, T. Szyperski, ChemPhysChem 2014, 15, 18721879.

[44] a) L. Frydman, A. Lupulescu, T. Scherf, J. Am. Chem. Soc. 2003, 125, 9204-9217 b) Y. Shrot, L. Frydman, J. Chem. Phys. 2009, 131, 224516.

[45] L. Castañar, Magn. Reson. Chem. 2017, 55, 47-53.

[46] J. Wist, Magn. Reson. Chem. 2017, 55, 22-28.

[47] a) K. Zangger, Prog. Nucl. Magn. Reson. Spectrosc. 2015, 86-87, 1-20 b) L. Castañar, T. Parella, Magn. Reson. Chem. 2015, 53, 399-426.

[48] J. A. Aguilar, S. Faulkner, M. Nilsson, G. A. Morris, Angew. Chem. 2010, 122, 3993-3995, Angew. Chem. Int. Ed. 2010, 49, 3901-3903.

[49] K. Zangger, H. Sterk, J. Magn. Reson. 1997, 124, 486-489.

[50] M. Nilsson, G. A. Morris, Chem. Commun. 2007, 933-935.

[51] L. Kaltschnee, A. Kolmer, I. Timari, V. Schmidts, R. W. Adams, M. Nilsson, K. E. Kover, G. A. Morris, C. M. Thiele, Chem. Commun. 2014, 50, 15702-15705.

[52] J. A. Aguilar, A. A. Colbourne, J. Cassani, M. Nilsson, G. A. Morris, Angew. Chem. 2012, 124, 6566-6569, Angew. Chem. Int. Ed. 2012, 51, 6460-6463.

[53] P. Trigo-Mouriño, C. Merle, M. R. M. Koos, B. Luy, R. R. Gil, Chem. Eur. J. 2013, 19, 7013-7019.

[54] a) B. T. Martin, G. C. Chingas, O. M. McDougal, J. Magn. Reson. 2012, 218, 147-152 b) P. C. Stein, M. di Cagno, A. Bauer-Brandl, Pharm. Res. 2011, 28, 2140-2146.

[55] a) J. Sangster, J. Phys. Chem. Ref. Data 1989, 18, 1111-1229 b) A. Noble, J. Chromatogr., A 1993, 642, 3-14 c) A. Finizio, M. Vighi, D. Sandroni, Chemosphere 1997, 34, 131-161.

[56] A. Bax, R. Freeman, J. Magn. Reson. 1980, 37, 177-181.

[57] J. Kind, C. M. Thiele, J. Magn. Reson. 2015, 260, 109-115.

[58] D. Neuhaus, in eMagRes, John Wiley \& Sons, 2007.

[59] M. J. Minch, Conc. Magn. Reson. 1994, 6, 41-56.

[60] a) C. H. Wu, A. Ramamoorthy, S. J. Opella, J. Magn. Reson. 1994, 109, 270-272 b) A. Lesage, Phys. Chem. Chem. Phys. 2009, 11, 6876-6891 c) P. Hodgkinson, in Annu. Rep. NMR Spectrosc., Vol. Volume 72 (Ed.: A. W. Graham), Academic Press, 2011, pp. 185-223. 
[61] a) S. E. Ashbrook, M. J. Duer, Concepts Magn. Reson. A 2006, 28A, 183-248 b) P. P. Man, in Encyclopedia of Analytical Chemistry, John Wiley \& Sons, 2006 c) T. Bräuniger, M. Jansen, Z. Anorg. Allg. Chem. 2013, 639, 857-879.

[62] a) D. Doddrell, V. Glushko, A. Allerhand, J. Chem. Phys. 1972, 56, 3683-3689 b) A. Kumar, R. R. Ernst, K. Wüthrich, Biochem. Biophys. Res. Commun. 1980, 95, 1-6 c) J. Tropp, J. Chem. Phys. 1980, 72, 6035-6043 d) J. Anglister, G. Srivastava, F. Naider, Prog. Nucl. Magn. Reson. Spectrosc. 2016, 97, 40-56.

[63] a) A. Saupe, G. Englert, Phys. Rev. Lett. 1963, 11, 462-464 b) M. J. Freiser, Phys. Rev. Lett. 1970, 24, 10411043 c) G. N. Patey, E. E. Burnell, J. G. Snijders, C. A. de Lange, Chem. Phys. Lett. 1983, 99, 271-274 d) L. A. Madsen, T. J. Dingemans, M. Nakata, E. T. Samulski, Phys. Rev. Lett. 2004, 92, 145505.

[64] F. Kramer, M. V. Deshmukh, H. Kessler, S. J. Glaser, Concepts Magn. Reson. A 2004, 21A, 10-21.

[65] a) J.-C. Hus, D. Marion, M. Blackledge, J. Am. Chem. Soc. 2001, 123, 1541-1542 b) Y.-S. Jung, M. Zweckstetter, J. Biomol. NMR 2004, 30, 25-35 c) J. H. Prestegard, K. L. Mayer, H. Valafar, G. C. Benison, in Methods Enzymol., Vol. Volume 394, Academic Press, 2005, pp. 175-209 d) G. Nodet, L. Salmon, V. Ozenne, S. Meier, M. R. Jensen, M. Blackledge, J. Am. Chem. Soc. 2009, 131, 17908-17918.

[66] C. M. Thiele, Eur. J. Org. Chem. 2008, 2008, 5673-5685.

[67] M. H. Levitt, Spin Dynamics: Basics of Nuclear Magnetic Resonance, John Wiley \& Sons, Weinheim, 2001.

[68] A. Enthart, J. C. Freudenberger, J. Furrer, H. Kessler, B. Luy, J. Magn. Reson. 2008, 192, 314-322.

[69] a) L. Verdier, P. Sakhaii, M. Zweckstetter, C. Griesinger, J. Magn. Reson. 2003, 163, 353-359 b) N. Nath, E. J. d'Auvergne, C. Griesinger, Angew. Chem. 2015, 127, 12897-12901 Angew. Chem. Int. Ed. 2015, 54, 1270612710 .

[70] a) R. Tycko, F. J. Blanco, Y. Ishii, J. Am. Chem. Soc. 2000, 122, 9340-9341 b) R. S. Lipsitz, N. Tjandra, Annu. Rev. Biophys. Biomol. Struct. 2004, 33, 387-413 c) J. H. Prestegard, C. M. Bougault, A. I. Kishore, Chem. Rev. 2004, 104, 3519-3540.

[71] a) R. M. Gschwind, Angew. Chem. 2005, 117, 4744-4746, Angew. Chem. Int. Ed. 2005, 44, 4666-4668 b) G. Kummerlöwe, B. Luy, TrAC, Trends Anal. Chem. 2009, 28, 483-493.

[72] a) M. H. Bailor, C. Musselman, A. L. Hansen, K. Gulati, D. J. Patel, H. M. Al-Hashimi, Nat. Protocols 2007, 2, 1536-1546 b) C. Tripathy, J. Zeng, P. Zhou, B. R. Donald, Proteins: Struct., Funct., Bioinf. 2012, 80, 433-453 c) L. Salmon, G. Bascom, I. Andricioaei, H. M. Al-Hashimi, J. Am. Chem. Soc. 2013, 135, 5457-5466.

[73] a) A. Grishaev, J. Wu, J. Trewhella, A. Bax, J. Am. Chem. Soc. 2005, 127, 16621-16628 b) F. Gabel, B. Simon, M. Nilges, M. Petoukhov, D. Svergun, M. Sattler, J. Biomol. NMR 2008, 41, 199 c) A. Grishaev, V. Tugarinov, L. E. Kay, J. Trewhella, A. Bax, J. Biomol. NMR 2008, 40, 95-106 d) S. Esteban-Martín, R. B. Fenwick, X. Salvatella, J. Am. Chem. Soc. 2010, 132, 4626-4632.

[74] a) K. Fredriksson, M. Louhivuori, P. Permi, A. Annila, J. Am. Chem. Soc. 2004, 126, 12646-12650 b) S. Esteban-Martín, R. Bryn Fenwick, X. Salvatella, WIREs Comput. Mol. Sci. 2012, 2, 466-478.

[75] a) J. Yan, F. Delaglio, A. Kaerner, A. D. Kline, H. Mo, M. J. Shapiro, T. A. Smitka, G. A. Stephenson, E. R. Zartler, J. Am. Chem. Soc. 2004, 126, 5008-5017 b) B. Luy, K. Kobzar, S. Knör, J. Furrer, D. Heckmann, H. Kessler, J. Am. Chem. Soc. 2005, 127, 6459-6465 c) U. M. Reinscheid, M. Köck, C. Cychon, V. Schmidts, C. M. Thiele, C. Griesinger, Eur. J. Org. Chem. 2010, 2010, 6900-6903 d) V. M. R. Kakita, K. Rachineni, J. 
Bharatam, J. Mol. Struct. 2013, 1053, 122-126 e) C. S. Lancefield, A. M. Z. Slawin, N. J. Westwood, T. Lebl, Magn. Reson. Chem. 2015, 53, 467-475.

[76] G. Kummerlöwe, B. Crone, M. Kretschmer, S. F. Kirsch, B. Luy, Angew. Chem. 2011, 123, 2693-2696 Angew. Chem. Int. Ed. $2011,50,2643-2645$.

[77] H. F. Azurmendi, C. A. Bush, J. Am. Chem. Soc. 2002, 124, 2426-2427.

[78] M. Zweckstetter, A. Bax, J. Am. Chem. Soc. 2000, 122, 3791-3792.

[79] a) L. Salmon, G. M. Giambaşu, E. N. Nikolova, K. Petzold, A. Bhattacharya, D. A. Case, H. M. Al-Hashimi, J. Am. Chem. Soc. 2015, 137, 12954-12965 b) A. De Simone, R. W. Montalvao, C. M. Dobson, M. Vendruscolo, Biochemistry 2013, 52, 6480-6486 c) M. D. Mukrasch, P. Markwick, J. Biernat, M. von Bergen, P. Bernadó, C. Griesinger, E. Mandelkow, M. Zweckstetter, M. Blackledge, J. Am. Chem. Soc. 2007, 129, 5235-5243 d) M. Martín-Pastor, A. Canales, F. Corzana, J. L. Asensio, J. Jiménez-Barbero, J. Am. Chem. Soc. 2005, 127, 35893595 e) A. Almond, J. B. Axelsen, J. Am. Chem. Soc. 2002, 124, 9986-9987.

[80] a) A. Pizzirusso, M. E. Di Pietro, G. De Luca, G. Celebre, M. Longeri, L. Muccioli, C. Zannoni, ChemPhysChem 2014, 15, 1356-1367 b) A. C. J. Weber, E. E. Burnell, W. L. Meerts, C. A. de Lange, R. Y. Dong, L. Muccioli, A. Pizzirusso, C. Zannoni, J. Chem. Phys. 2015, 143, 011103.

[81] M. Zweckstetter, Nat. Protocols 2008, 3, 679-690.

[82] H. Valafar, J. H. Prestegard, J. Magn. Reson. 2004, 167, 228-241.

[83] A. Navarro-Vázquez, Magn. Reson. Chem. 2012, 50, S73-S79.

[84] Y. Millot, P. P. Man, Concepts Magn. Reson. A 2012, 40A, 215-252.

[85] D. Massiot, F. Fayon, M. Capron, I. King, S. Le Calvé, B. Alonso, J.-O. Durand, B. Bujoli, Z. Gan, G. Hoatson, Magn. Reson. Chem. 2002, 40, 70-76.

[86] M. Bak, J. T. Rasmussen, N. C. Nielsen, J. Magn. Reson. 2000, 147, 296-330.

[87] a) M. Hartung, H. Günther, J.-P. Amoureux, C. Fernandéz, Magn. Reson. Chem. 1998, 36, S61-S70 b) A. Pepels, H. Günther, J.-P. Amoureux, C. Fernandéz, J. Am. Chem. Soc. 2000, 122, 9858-9859.

[88] a) I. Canet, J. Courtieu, A. Loewenstein, A. Meddour, J. M. Pechine, J. Am. Chem. Soc. 1995, 117, 6520-6526 b) P. Lesot, D. Merlet, A. Loewenstein, J. Courtieu, Tetrahedron: Asymmetry 1998, 9, 1871-1881 c) P. Lesot, M. Sarfati, J. Courtieu, Chem. Eur. J. 2003, 9, 1724-1745 d) L. Ziani, P. Lesot, A. Meddour, J. Courtieu, Chem. Commun. 2007, 4737-4739.

[89] P. Tzvetkova, B. Luy, Magn. Reson. Chem. 2016, 54, 351-357.

[90] a) J. R. Tolman, J. M. Flanagan, M. A. Kennedy, J. H. Prestegard, Proc. Natl. Acad. Sci. U. S. A. 1995, 92, 9279-9283 b) N. Tjandra, A. Bax, Science 1997, 278, 1111-1114 c) N. Tjandra, J. G. Omichinski, A. M. Gronenborn, G. M. Clore, A. Bax, Nat. Struct. Mol. Biol. 1997, 4, 732-738.

[91] H.-J. Sass, G. Musco, S. Stahl, P. Wingfield, S. Grzesiek, J. Biomol. NMR 2000, 18, 303-309.

[92] B. Deloche, E. T. Samulski, Macromolecules 1981, 14, 575-581.

[93] a) B. Luy, K. Kobzar, H. Kessler, Angew. Chem. 2004, 116, 1112-1115, Angew. Chem. Int. Ed. 2004, 43, 10921094 b) G. Kummerlöwe, J. Auernheimer, A. Lendlein, B. Luy, J. Am. Chem. Soc. 2007, 129, 6080-6081. 
[94] K. Kobzar, H. Kessler, B. Luy, Angew. Chem. 2005, 117, 3205-3207, Angew. Chem. Int. Ed. 2005, 44, 35093509.

[95] J. C. Freudenberger, P. Spiteller, R. Bauer, H. Kessler, B. Luy, J. Am. Chem. Soc. 2004, 126, 14690-14691.

[96] N. Nath, M. Schmidt, R. R. Gil, R. T. Williamson, G. E. Martin, A. Navarro-Vázquez, C. Griesinger, Y. Liu, J. Am. Chem. Soc. 2016, 138, 9548-9556.

[97] a) M. M. Meinholz, M. Klemmer, E. Kriemen, D. Stalke, Chem. Eur. J. 2011, 17, 9415-9422 b) C. Maaß, D. M. Andrada, R. A. Mata, R. Herbst-Irmer, D. Stalke, Inorg. Chem. 2013, 52, 9539-9548 c) A. Salomone, F. M. Perna, A. Falcicchio, S. O. Nilsson Lill, A. Moliterni, R. Michel, S. Florio, D. Stalke, V. Capriati, Chem. Sci. 2014, 5, 528-538 d) D.-R. Dauer, M. Flügge, R. Herbst-Irmer, D. Stalke, Dalton Trans. 2016, 45, 6136-6148.

[98] a) M. I. Hrovat, C. G. Wade, J. Magn. Reson. 1981, 44, $62-75$ b) M. Holz, S. R. Heil, A. Sacco, Phys. Chem. Chem. Phys. 2000, 2, 4740-4742.

[99] a) S. Braun, H.-O. Kalinowski, S. Berger, 100 and more basic NMR experiments, Wiley VCH Weinheim, 1996 b) S. J. Seedhouse, M. M. Hoffmann, J. Chem. Educ. 2008, 85, 836 c) N. N. Yadav, A. M. Torres, W. S. Price, J. Magn. Reson. 2008, 194, 25-28.

[100]D. F. Evans, J. Chem. Soc. 1959, 2003-2005.

[101] L. Emsley, G. Bodenhausen, Chem. Phys. Lett. 1990, 165, 469-476.

[102] a) Z. Rappoport, I. Marek, The Chemistry of Organolithium Compounds, Volume 1, 2 Volume Set, John Wiley \& Sons, Weinheim, 2004 b) H. Günther, in eMagRes, John Wiley \& Sons, 2007.

[103] a) H. Gilman, A. H. Haubein, H. Hartzfeld, J. Org. Chem. 1954, 19, 1034-1040 b) H. Gilman, B. J. Gaj, J. Org. Chem. 1957, 22, 1165-1168 c) P. Stanetty, M. D. Mihovilovic, J. Org. Chem. 1997, 62, 1514-1515.

[104] C. Strohmann, V. H. Gessner, Angew. Chem. 2007, 119, 4650-4653, Angew. Chem. Int. Ed. 2007, 46, 45664569.

[105]a) G. W. Klumpp, H. Luitjes, M. Schakel, F. J. J. de Kanter, R. F. Schmitz, N. J. R. Van Eikema Hommes, Angew. Chem. 1992, 104, 624-626, Angew. Chem. Int. Ed. 1992, 31, 633-635 b) H. Luitjes, M. Schakel, M. P. Aarnts, R. F. Schmitz, F. J. J. de Kanter, G. W. Klumpp, Tetrahedron 1997, 53, 9977-9988.

[106]D. Margerison, J. P. Newport, Trans. Faraday Soc. 1963, 59, 2058-2063.

[107]a) N. A. Peppas, P. Bures, W. Leobandung, H. Ichikawa, Eur. J. Pharm. Biopharm. 2000, 50, 27-46 b) C.-C. Lin, A. T. Metters, Adv. Drug Delivery Rev. 2006, 58, 1379-1408 c) T. R. Hoare, D. S. Kohane, Polymer 2008, 49, 1993-2007 d) E. M. Ahmed, J. Adv. Res. 2015, 6, 105-121.

[108] a) J. M. Zielinski, J. L. Duda, AIChE J. 1992, 38, 405-415 b) B. Amsden, Macromolecules 1998, 31, 8382-8395 c) S. C. George, S. Thomas, Prog. Polym. Sci. 2001, 26, 985-1017.

[109] a) M. J. Hynes, J. Chem. Soc., Dalton Trans. 1993, 311-312 b) I. W. Wyman, D. H. Macartney, Org. Biomol. Chem. 2008, 6, 1796-1801 c) P. Thordarson, Chem. Soc. Rev. 2011, 40, 1305-1323 d) J. S. Renny, L. L. Tomasevich, E. H. Tallmadge, D. B. Collum, Angew. Chem. 2013, 125, 12218-12234, Angew. Chem. Int. Ed. 2013, 52, 11998-12013.

[110] a) M. C. Masiker, C. L. Mayne, E. M. Eyring, Magn. Reson. Chem. 2006, 44, 220-229 b) M. C. Masiker, C. L. Mayne, B. J. Boone, A. M. Orendt, E. M. Eyring, Magn. Reson. Chem. 2010, 48, 94-100. 
[111] a) C. J. Pedersen, H. K. Frensdorff, Angew. Chem. 1972, 84, 16-26, Angew. Chem. Int. Ed. 1972, 11, 16-25 b) C. J. Pedersen, Angew. Chem. 1988, 100, 1053-1059, Angew. Chem. Int. Ed. 1988, 27, 1021-1027.

[112] a) B. Valeur, I. Leray, Coord. Chem. Rev. 2000, 205, 3-40 b) G. W. Gokel, W. M. Leevy, M. E. Weber, Chem. Rev. 2004, 104, 2723-2750.

[113] a) D. Landini, F. Montanari, F. M. Pirisi, J. Chem. Soc., Chem. Commun. 1974, 879-880 b) P. E. Stott, J. S. Bradshaw, W. W. Parish, J. Am. Chem. Soc. 1980, 102, 4810-4815.

[114] P. Giraudeau, Magn. Reson. Chem. 2017, 55, 61-69.

[115]Y. Mitrev, S. Simova, D. Jeannerat, Chem. Commun. 2016, 52, 5418-5420.

[116] R. J. Wieme, Clin. Chim. Acta 1959, 4, 317-321.

[117]a) K. Albert, J. Chromatogr., A 1995, 703, 123-147 b) V. Exarchou, M. Krucker, T. A. van Beek, J. Vervoort, I. P. Gerothanassis, K. Albert, Magn. Reson. Chem. 2005, 43, 681-687.

[118] a) S. Caldarelli, Magn. Reson. Chem. 2007, 45, S48-S55 b) R. E. Hoffman, H. Arzuan, C. Pemberton, A. Aserin, N. Garti, J. Magn. Reson. 2008, 194, 295-299 c) M.-V. Salvia, F. Ramadori, S. Springhetti, M. DiezCastellnou, B. Perrone, F. Rastrelli, F. Mancin, J. Am. Chem. Soc. 2015, 137, 886-892 d) G. Pages, C. Delaurent, S. Caldarelli, Angew. Chem. 2006, 118, 6096-6099, Angew. Chem. Int. Ed. 2006, 45, 5950-5953.

[119]a) R. Evans, I. J. Day, RSC Advances 2016, 6, 47010-47022 b) N. V. Gramosa, N. M. S. P. Ricardo, R. W. Adams, G. A. Morris, M. Nilsson, Magn. Reson. Chem. 2016, 54, 815-820.

[120]A. Fick, Ann. Phys. 1855, 170, 59-86.

[121]J. Crank, The Mathematics of Diffusion, 2nd Edition ed., Oxford University Press, London, 1975.

[122] a) H. Y. Carr, E. M. Purcell, Phys. Rev. 1954, 94, 630-638 b) S. Meiboom, D. Gill, Rev. Sci. Instrum. 1958, 29, 688-691.

[123] a) J. H. Strange, M. Rahman, E. G. Smith, Phys. Rev. Lett. 1993, 71, 3589-3591 b) E. W. Hansen, G. Fonnum, E. Weng, J. Phys. Chem. B 2005, 109, 24295-24303 c) J. P. Korb, New J. Phys. 2011, 13, 035016.

[124] S. W. Provencher, Comput. Phys. Commun. 1982, 27, 213-227.

[125] a) L. Venkataramanan, S. Yi-Qiao, M. D. Hurlimann, IEEE Trans. Signal Process. 2002, 50, 1017-1026 b) Y. Q. Song, L. Venkataramanan, M. D. Hürlimann, M. Flaum, P. Frulla, C. Straley, J. Magn. Reson. 2002, 154, 261-268.

[126] A. C. F. Ribeiro, M. C. F. Barros, L. M. P. Verissimo, V. M. M. Lobo, A. J. M. Valente, J. Solution Chem. 2014, 43, 83-92.

[127] a) R.-l. Wu, T. Li, E. Nies, Chin. J. Polym. Sci. 2013, 31, 21-38 b) A. Gautieri, S. Vesentini, A. Redaelli, Journal of Molecular Modeling 2010, 16, 1845-1851 c) Y. Wu, S. Joseph, N. R. Aluru, J. Phys. Chem. B 2009, 113, 3512-3520.

[128] C. Farès, J. Hassfeld, D. Menche, T. Carlomagno, Angew. Chem. 2008, 120, 3782-3786, Angew. Chem. Int. Ed. 2008, $47,3722-3726$.

[129] A. A. Al-Massaedh, M. Schmidt, U. Pyell, U. M. Reinscheid, ChemistryOpen 2016, 5, 455-459. 
[130]W. Maier, A. Saupe, Z. Naturforsch. A 1959, 14, 882

[131] a) E. E. Burnell, R. Berardi, R. T. Syvitski, C. Zannoni, Chem. Phys. Lett. 2000, 331, 455-464 b) R. T. Syvitski, E. E. Burnell, J. Chem. Phys. 2000, 113, 3452-3465 c) T. Dingemans, D. J. Photinos, E. T. Samulski, A. F. Terzis, C. Wutz, J. Chem. Phys. 2003, 118, 7046-7061 d) J. M. Polson, in NMR of Ordered Liquids (Eds.: E. E. Burnell, C. A. de Lange), Springer Netherlands, Dordrecht, 2003, pp. 325-344 e) M. Pavanello, B. Mennucci, A. Ferrarini, J. Chem. Phys. 2005, 122, 064906 f) C. Benzi, V. Barone, R. Tarroni, C. Zannoni, J. Chem. Phys. 2006, 125, 174904 g) E. Elliott Burnell, L. C. ter Beek, Z. Sun, J. Chem. Phys. 2008, 128, 164901 h) Z. Danilović, E. E. Burnell, J. Chem. Phys. 2009, 130, 154506 i) R. O. Sokolovskii, E. E. Burnell, J. Chem. Phys. 2009, 130, 154507 j) F. Li, A. Grishaev, J. Ying, A. Bax, J. Am. Chem. Soc. 2015, 137, 14798-14811.

[132]A. O. Frank, J. C. Freudenberger, A. K. Shaytan, H. Kessler, B. Luy, Magn. Reson. Chem. 2015, 53, $213-217$.

[133] M. Ottiger, A. Bax, J. Am. Chem. Soc. 1999, 121, 4690-4695.

[134] M. Ottiger, F. Delaglio, J. L. Marquardt, N. Tjandra, A. Bax, J. Magn. Reson. 1998, 134, 365-369.

[135]C. A. de Lange, E. E. Burnell, in NMR of Ordered Liquids (Eds.: E. E. Burnell, C. A. de Lange), Springer Netherlands, Dordrecht, 2003, pp. 5-26.

[136] a) C. L. Lawson, R. J. Hanson, Solving least squares problems, Vol. 15, SIAM, 1995 b) P. F. Dubois, K. Hinsen, J. Hugunin, Comput. Phys. 1996, 10, 262-267.

[137] a) C. A. Hunter, J. K. M. Sanders, J. Am. Chem. Soc. 1990, 112, 5525-5534 b) M. J. Rashkin, M. L. Waters, J. Am. Chem. Soc. 2002, 124, 1860-1861 c) M. L. Waters, Curr. Opin. Chem. Biol. 2002, 6, 736-741 d) E. A. Meyer, R. K. Castellano, F. Diederich, Angew. Chem. 2003, 115, 1244-1287, Angew. Chem. Int. Ed. 2003, 42, 1210-1250 e) M. O. Sinnokrot, C. D. Sherrill, J. Am. Chem. Soc. 2004, 126, 7690-7697 f) C. R. Martinez, B. L. Iverson, Chem. Sci. 2012, 3, 2191-2201.

[138] C. M. Breneman, K. B. Wiberg, J. Comput. Chem. 1990, 11, 361-373.

[139]a) H. J. C. Berendsen, D. van der Spoel, R. van Drunen, Comput. Phys. Commun. 1995, 91, 43-56 b) D. Van Der Spoel, E. Lindahl, B. Hess, G. Groenhof, A. E. Mark, H. J. C. Berendsen, J. Comput. Chem. 2005, 26, 1701-1718 c) B. Hess, C. Kutzner, D. van der Spoel, E. Lindahl, J. Chem. Theory Comput. 2008, 4, 435-447 d) S. Pronk, S. Páll, R. Schulz, P. Larsson, P. Bjelkmar, R. Apostolov, M. R. Shirts, J. C. Smith, P. M. Kasson, D. van der Spoel, B. Hess, E. Lindahl, Bioinformatics 2013, 29, 845-854 e) M. J. Abraham, T. Murtola, R. Schulz, S. Páll, J. C. Smith, B. Hess, E. Lindahl, SoftwareX 2015, 1-2, 19-25.

[140] a) W. L. Jorgensen, D. S. Maxwell, J. Tirado-Rives, J. Am. Chem. Soc. 1996, 118, 11225-11236 b) G. Kaminski, W. L. Jorgensen, J. Phys. Chem. 1996, 100, 18010-18013 c) G. A. Kaminski, R. A. Friesner, J. Tirado-Rives, W. L. Jorgensen, J. Phys. Chem. B 2001, 105, 6474-6487 d) M. L. P. Price, D. Ostrovsky, W. L. Jorgensen, J. Comput. Chem. 2001, 22, 1340-1352.

[141]H. J. C. Berendsen, J. P. M. Postma, W. F. van Gunsteren, A. DiNola, J. R. Haak, J. Chem. Phys. 1984, 81, 3684-3690.

[142] M. Brehm, B. Kirchner, J. Chem. Inf. Model. 2011, 51, 2007-2023.

[143] a) G. Wagner, A. Pardi, K. Wuethrich, J. Am. Chem. Soc. 1983, 105, 5948-5949 b) C. L. Perrin, J. B. Nielson, Annu. Rev. Phys. Chem. 1997, 48, 511-544 c) T. Steiner, Angew. Chem. 2002, 114, 50-80, Angew. Chem. Int. Ed. 2002, 41, 48-76 d) P. Gilli, L. Pretto, V. Bertolasi, G. Gilli, Acc. Chem. Res. 2009, 42, 33-44.

[144]H. D. Flack, G. Bernardinelli, Chirality 2008, 20, 681-690. 
[145]J. P. Linge, M. Habeck, W. Rieping, M. Nilges, Bioinformatics 2003, 19, 315-316.

[146] a) J. G. Napolitano, J. A. Gavín, C. García, M. Norte, J. J. Fernández, A. Hernández Daranas, Chem. Eur. J. 2011, 17, 6338-6347 b) G. Bifulco, P. Dambruoso, L. Gomez-Paloma, R. Riccio, Chem. Rev. 2007, 107, 37443779 .

[147] M. Karplus, J. Am. Chem. Soc. 1963, 85, 2870-2871.

[148] W. Liu, D. Zell, M. John, L. Ackermann, Angew. Chem. 2015, 127, 4165-4169, Angew. Chem. Int. Ed. 2015, $54,4092-4096$.

[149] a) L. Kiss, F. Fülöp, Chem. Rev. 2013, 114, 1116-1169 b) D. Seebach, J. Gardiner, Acc. Chem. Res. 2008, 41, 1366-1375 c) F. Gnad, O. Reiser, Chem. Rev. 2003, 103, 1603-1624.

[150] R. Berger, J. Courtieu, R. R. Gil, C. Griesinger, M. Köck, P. Lesot, B. Luy, D. Merlet, A. Navarro-Vázquez, M. Reggelin, U. M. Reinscheid, C. M. Thiele, M. Zweckstetter, Angew. Chem. 2012, 124, 8512-8515, Angew. Chem. Int. Ed. 2012, 51, 8388-8391.

[151] N. L. Allinger, J. Am. Chem. Soc. 1977, 99, 8127-8134.

[152] T. A. Halgren, J. Comput. Chem. 1996, 17, 490-519.

[153] G. M. Clore, C. D. Schwieters, J. Am. Chem. Soc. 2003, 125, 2902-2912.

[154] a) F. Neese, WIREs Comput. Mol. Sci. 2012, 2, 73-78 b) S. Grimme, S. Ehrlich, L. Goerigk, J. Comput. Chem. 2011, 32, 1456-1465 c) S. Grimme, J. Antony, S. Ehrlich, H. Krieg, J. Chem. Phys. 2010, 132, 154104 d) F. Weigend, R. Ahlrichs, Phys. Chem. Chem. Phys. 2005, 7, 3297-3305 e) A. Schäfer, H. Horn, R. Ahlrichs, J. Chem. Phys. 1992, 97, 2571-2577.

[155] J. A. Losonczi, M. Andrec, M. W. Fischer, J. H. Prestegard, J. Magn. Reson. 1999, 138, 334-342.

[156] G. Cornilescu, J. L. Marquardt, M. Ottiger, A. Bax, J. Am. Chem. Soc. 1998, 120, 6836-6837.

[157]a) R. Steyn, H. Z. Sable, Tetrahedron 1971, 27, 4429-4447 b) A. Das, K. K. Mahato, S. S. Panja, T. Chakraborty, J. Chem. Phys. 2003, 119, 2523-2530 c) P. K. Argawal, H.-J. Schneider, M. S. Malik, S. N. Rastogi, Org. Magn. Resonance 1983, 21, 146-150.

[158] C. M. Thiele, V. Schmidts, B. Böttcher, I. Louzao, R. Berger, A. Maliniak, B. Stevensson, Angew. Chem. 2009, 121, 6836-6840, Angew. Chem. Int. Ed. 2009, 48, 6708-6712.

[159]A. A. Shahkhatuni, A. G. Shahkhatuni, H. A. Panosyan, A. B. Sahakyan, I.-J. L. Byeon, A. M. Gronenborn, Magn. Reson. Chem. 2007, 45, 557-563.

[160] S. S. Shapiro, M. B. Wilk, Biometrika 1965, 52, 591-611.

[161] a) L. Frydman, Annu. Rev. Phys. Chem. 2001, 52, 463-498 b) S. E. Ashbrook, Phys. Chem. Chem. Phys. 2009, 11, 6892-6905 c) C. Fernandez, M. Pruski, in Solid State NMR (Ed.: J. C. C. Chan), Springer Berlin Heidelberg, Berlin, Heidelberg, 2012, pp. 119-188.

[162] a) R. W. Schurko, I. Hung, C. L. B. Macdonald, A. H. Cowley, J. Am. Chem. Soc. 2002, 124, 13204-13214 b) J. Autschbach, S. Zheng, R. W. Schurko, Concepts Magn. Reson. A 2010, 36A, 84-126.

[163] a) T. Wiegand, H. Eckert, O. Ekkert, R. Fröhlich, G. Kehr, G. Erker, S. Grimme, J. Am. Chem. Soc. 2012, 134, 4236-4249 b) J. W. E. Weiss, D. L. Bryce, J. Phys. Chem. A 2010, 114, 5119-5131 c) P. J. Pallister, I. L. 
Moudrakovski, J. A. Ripmeester, Phys. Chem. Chem. Phys. 2009, 11, 11487-11500 d) S. E. Ashbrook, A. J. Berry, D. J. Frost, A. Gregorovic, C. J. Pickard, J. E. Readman, S. Wimperis, J. Am. Chem. Soc. 2007, 129, 13213-13224 e) J. A. Tang, J. D. Masuda, T. J. Boyle, R. W. Schurko, ChemPhysChem 2006, 7, 117-130 f) C. Gervais, R. Dupree, K. J. Pike, C. Bonhomme, M. Profeta, C. J. Pickard, F. Mauri, J. Phys. Chem. A 2005, 109, 6960-6969 g) C. M. Widdifield, I. Moudrakovski, D. L. Bryce, Phys. Chem. Chem. Phys. 2014, 16, 1334013359 h) Z. Falls, E. Zurek, J. Autschbach, Phys. Chem. Chem. Phys. 2016, 18, 24106-24118.

[164] a) H. Yu, X. Tan, G. M. Bernard, V. V. Terskikh, J. Chen, R. E. Wasylishen, J. Phys. Chem. A 2015, 119, 82798293 b) B. E. G. Lucier, J. A. Tang, R. W. Schurko, G. A. Bowmaker, P. C. Healy, J. V. Hanna, J. Phys. Chem. C 2010, 114, 7949-7962 c) H. Hamaed, M. W. Laschuk, V. V. Terskikh, R. W. Schurko, J. Am. Chem. Soc. 2009, 131, 8271-8279 d) J. A. Tang, B. D. Ellis, T. H. Warren, J. V. Hanna, C. L. B. Macdonald, R. W. Schurko, J. Am. Chem. Soc. 2007, 129, 13049-13065 e) K. J. Ooms, V. V. Terskikh, R. E. Wasylishen, J. Am. Chem. Soc. 2007, 129, 6704-6705.

[165]a) S. Bachmann, B. Gernert, D. Stalke, Chem. Commun. 2016, 52, 12861-12864 b) D. Johnels, A. Boman, U. Edlund, Magn. Reson. Chem. 1998, 36, S151-S156 c) L. A. Paquette, W. Bauer, M. R. Sivik, M. Buehl, M. Feigel, P. v. R. Schleyer, J. Am. Chem. Soc. 1990, 112, 8776-8789 d) P. Jutzi, E. Schlüter, C. Krüger, S. Pohl, Angew. Chem. 1983, 95, 1015-1016, Angew. Chem. Int. Ed. 1983, 22, 994-994 e) D. Stalke, Angew. Chem. 1994, 106, 2256-2259, Angew. Chem. Int. Ed. 1994, 33, 2168-2171 f) T. P. Hanusa, Organometallics 2002, 21, 2559-2571 g) P. Jutzi, N. Burford, Chem. Rev. 1999, 99, 969-990 h) W. Kaminsky, A. Funck, H. Hahnsen, Dalton Trans. 2009, 8803-8810 i) E. W. Neuse, J. Inorg. Organomet. Polym. Mater. 2005, 15, 3-31 j) W. Kaminsky, Macromol. Chem. Phys. 1996, 197, 3907-3945 k) H. Sinn, W. Kaminsky, H.-J. Vollmer, R. Woldt, Angew. Chem. 1980, 92, 396-402, Angew. Chem. Int. Ed. 1980, 19, 390-392 1) H. Köpf, P. Köpf-Maier, Angew. Chem. 1979, 91, 509-509, Angew. Chem. Int. Ed. 1979, 18, 477-478.

[166]J. Clark Stewart, D. Segall Matthew, J. Pickard Chris, J. Hasnip Phil, I. J. Probert Matt, K. Refson, C. Payne Mike, Z. Kristallogr. -New Cryst. Struct. 2005, 220, 567.

[167]a) A. D. Becke, J. Chem. Phys. 1993, 98, 5648-5652 b) C. Lee, W. Yang, R. G. Parr, Phys. Rev. B. 1988, 37, 785789 c) W. J. Hehre, R. Ditchfield, J. A. Pople, J. Chem. Phys. 1972, 56, 2257-2261.

[168] a) M. Perez, Magn. Reson. Chem. 2017, 55, 15-21 b) P. Güntert, Eur. Biophys. J. 2008, 38, 129.

[169] a) R. Tycko, F. J. Blanco, Y. Ishii, J. Am. Chem. Soc. 2000, 122, 9340-9341 b) C. Gayathri, N. V. Tsarevsky, R. R. Gil, Chem. Eur. J. 2010, 16, 3622-3626.

[170] a) G. A. Petersson, M. A. Al - Laham, J. Chem. Phys. 1991, 94, 6081-6090 b) G. A. Petersson, A. Bennett, T. G. Tensfeldt, M. A. Al - Laham, W. A. Shirley, J. Mantzaris, J. Chem. Phys. 1988, 89, 2193-2218.

[171]W. Humphrey, A. Dalke, K. Schulten, J. Mol. Graphics 1996, 14, 33-38.

[172] Wolfram Research, Inc., Mathematica, Version 11.0, Champaign, Illinois, 2016.

[173]J. Autschbach, Graphical Representation of Rank-2 Tensors can be found under ja01.chem.buffalo.edu/ jochena/tutorials/tutorials.html, 02.02. 2014. 


\section{DANKSAGUNG}

Ich bedanke mich bei meinem Doktorvater Prof. Dr. Dietmar Stalke für seine Betreuung und das Vertrauen in meine Arbeit. Prof. Dr. Franc Meyer danke ich für die Übernahme des Korreferats und sein Interesse an meiner Arbeit. Den weiteren Mitgliedern der Prüfungskommission danke ich ebenfalls für ihr Interesse an meiner Arbeit und die Zeit, die sie sich für die Begutachtung genommen haben. Prof. Stalke danke ich insbesondere auch für seine engagierte Förderung und seinen Einsatz, der mich letztlich zu einem Promotionsstipendium der Studienstiftung des deutschen Volkes geführt hat. Der Studienstiftung danke ich für ideelle und finanzielle Förderung.

An allen in dieser Arbeit vorgestellten Projekten hat Dr. Michael John durch interessante Diskussionen und kenntnisreiche Ratschläge größten Anteil genommen. Ihm, Ralf Schöne und der gesamten NMR Abteilung gilt mein ganz persönlicher und herzlicher Dank für ihre Unterstützung und Geduld.

Mitarbeiter, die durch praktische Arbeiten zu den Kapiteln beigetragen haben sind Jannis Gottwald und Christian Niklas (Qualitative Chromatography), Christian Steinmetzger (Alignment of Small Molecules, Structure Elucidation of Synthetic Indanes) und Anja Hausmann (Quantitative Analysis of Residual Quadrupolar Couplings). An weiteren unveröffentlichten Projekten haben Niklas Frerichs (Bestimmung des Wasser-Octanol Verteilungskoeffizienten) und Philipp Beyrich (Polymere als NMR Siegel für empfindliche Substanzen) gearbeitet. Ihnen allen danke ich vielmals für ihren Einsatz und hoffe, dass auch sie etwas unter meiner Betreuung gelernt haben. Prof. Dr. Lutz Ackermann und seinen Mitarbeitern Dr. Weiping Liu und Daniel Zell danke ich für ihre Mitarbeit bezüglich der synthetischen Indane. Prof. Dr. Ricardo A. Mata danke ich für seine Ratschläge zu DFT Rechnungen und MD Simulationen.

Sehr hilfreiche Korrekturvorschläge zu Teilen dieser Arbeit wurden von folgenden Personen gemacht: Jun.-Prof. Dr. Ann-Christin Pöppler, Dr. Sebastian Bachmann, Janine Wegner, Timo Schillmöller, Johannes Kretsch und Ingo Köhne. Vielen Dank!

Die Zeit im Labor und Büro war schön und dafür habe ich Sebastian Bachmann, Roman Neufeld, Ingo Köhne, Anne Kreyenschmidt und den oben erwähnten Praktikanten zu danken. Allen Mitgliedern der Arbeitsgruppe Stalke danke ich für eine wirklich angenehme Atmosphäre, große Hilfsbereitschaft und einen guten Zusammenhalt.

Freunde, mit denen ich abseits der Uni trainiert, gespielt und geredet habe, motivieren mich, jede weitere Hürde zu nehmen. Bei euch werde ich mich sicher noch mehr als einmal bedanken. Schließlich danke ich M. P. C. und J. und jedem der mich bis hierher ein Stück weit begleitet hat. 


\title{
CURRICULUM VitaE
}

\author{
Thomas Niklas
}

Zimmermannstraße 11

37075 Göttingen

${ }^{\star} 05.12 .1987$ in Olpe

\section{EDUCATION}

$11 / 2013-05 / 2017$

$09 / 2011-10 / 2013$

$10 / 2007-09 / 2011$

08/1998-06/2006
Ph. D. in Chemistry at the Georg-August-University Göttingen Thesis "Advanced NMR Techniques for the Investigation of Small Molecules in Solution"

M. Sc. in Chemistry (1.3, "with honors") at the Georg-AugustUniversity Göttingen

Thesis "Observation of Reactions in Polymers by NMR

Spectroscopy" (1.0)

B. Sc. in Chemistry (1.6) at the Georg-August-University

Göttingen

Thesis "Electrokinetic Measurements on Filtration Materials"

(Sartorius Stedim Biotech GmbH) (1.0)

A-level (2.3) at the St. Franziskus Gymnasium Olpe

(Skipping grade 10.1 to 11.2 )

\section{SCHOLARSHIPS AND AWARDS}

$10 / 2014-05 / 2017$

$09 / 2016$

$06 / 2015$
Ph. D. Scholarship by Studienstiftung des deutschen Volkes Summer School on NMR funded by Volkswagen Stiftung Student Grant Euromar 2015 


\section{TEACHING ACTIVITY}

10/2011-today

Experimental Chemistry I+II, Solids and Materials, Practical

NMR Spectroscopy, Methods in Chemistry I+II

\section{VARIOUS WORKING EXPERIENCES}

$\begin{array}{ll}\text { 04/2011-05/2011 } & \begin{array}{l}\text { Internship Sartorius Stedim Biotech GmbH } \\ \text { "Development of an Integrity Test for Depth Filter" } \\ \text { 06/2007-9/2007 }\end{array} \\ \text { Temporary Job at Sälzer Electric GmbH } \\ -09 / 2006-04 / 2007 & \text { Aide at intensive care unit at the St. Martinus Hospital Olpe } \\ & \text { Temporary job Sälzer Electric GmbH }\end{array}$

\section{VOLUNTARY SERVICES}

04/2015-today

$01 / 2015-2 / 2017$

$10 / 2009-12 / 2016$

$11 / 2015-03 / 2016$

$11 / 2013-10 / 2015$
Chairman and Coach Karate-Division Georg-August-

University Göttingen

Deputy Chairman Karate Dojo Göttingen

Coach Karate Dojo Göttingen

Supervision of a visually handicapped chemistry student

Supervision of an autistic chemistry student

\section{PERSONAL SKILLS}
Languages
German (mother tongue)
English (fluent)
Computer Skills
Scientific Data Handling
ORCA, Gromacs (basic knowldge)
Python (basic knowledge)
Expertise
Sachkundenachweis nach $₫ 5$ Chemikalienverbotsverordnung 


\section{CONFERENCES AND WORKSHOPS}

- $\quad$ Center of Materials Crystallography (CMC) meeting, 13.01.2017 in Göttingen (Talk)

- $\quad$ Summer School on the Theory of NMR financed by the Volkswagen Stiftung, 04.-10.09.2016

in Windischleuba (Workshop)

- $\quad$ 37. GDCh FGMR, 07.-10.09.2015 in Darmstadt (Poster)

- $\quad$ 18. NDDK, 07.-08.09.2015 in Goslar (Poster)

- $\quad$ Euromar, 05.-09.07.2015 in Prague (Poster, Travel Grant)

- $\quad$ Jungchemikerforum, 01.07.2015 in Göttingen (Poster)

- $\quad$ Center of Materials Crystallography (CMC) meeting, 28.03.2014 in Göttingen (Talk)

- $\quad$ 1. GNMR School, 13.-15.10.2014 in Munich (Workshop)

- $\quad$ EXPO-SIR Workshop, 10.-13.06.2014 in Bari (Workshop)

\section{SCIENTIFIC PUBLICATIONS}

1. T. Niklas, D. Stalke, M. John, "Single-shot titrations and reaction monitoring by slice-selective NMR spectroscopy" Chem. Commun., 2015. 51, 1275-1277.

2. T. Niklas, C. Steinmetzger, W. Liu, D. Zell, D. Stalke, L. Ackermann, M. John, "Determination of the Relative Configuration of $\beta$-Amino Acid Esters Based on Residual Dipolar Couplings" Eur. J. Org. Chem. 2015, 2015, 6801-6805. 